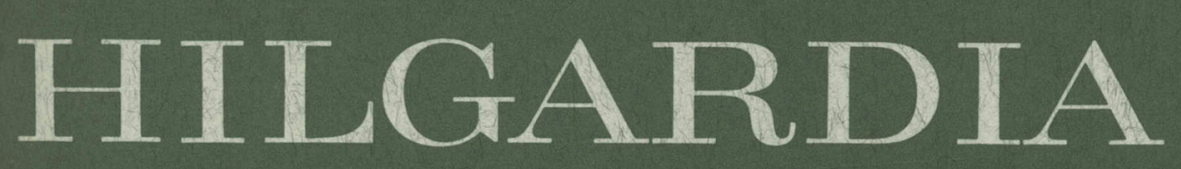

A JOURNAL OF AGRICULTURAL SCIENCE PUBLISHED BY THE CALIFORNIA AGRICULTURAL EXPER IMENT STATION

Volume 34, Number $11 \cdot$ August, 1963

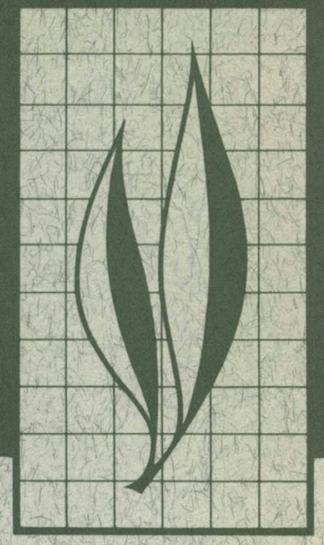

\title{
Theory of the Chemical Properties of Soil Colloidal Systems at Equilibrium
}

K. L. BABCOCK 


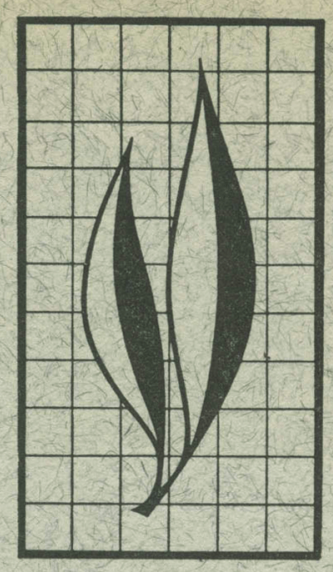

The theories of model soil colloids are developed in the present work. The principal point of departure is thermodynamics, and in the first section, an effort is made to present a consistent system of chemical thermodynamics and pertinent related physical chemistry. The second section deals with the Donnan theory and some consequences of it which may have relevance for soil systems. Electrochemical measurements are also discussed. In the third section, the Gouy theory of the electrical double layer is reviewed and the theory is used to calculate ionic activities. In the fourth section, ion-exchange equations based on various models are developed. The significance of different models for soil systems is analyzed in relation to the available data. In the fifth section, a discussion is presented of the thermodynamics of soil moisture. The emphasis throughout is on the development of theory, and no effort has been made to make historical or literature surveys.

See inside back cover for Contents.

\section{THE AUTHOR:}

Kenneth L. Babcock is Associate Professor of Soil Chemistry, Department of Soils and Plant Nutrition, Berkeley.

The author wishes to acknowledge gratefully the assistance of Professor Roy Overstreet who has read the entire text and made many helpful suggestions. 


\section{Theory of Chemical Properties of Soil Colloidal Systems at Equilibrium ${ }^{1}$}

\section{INTRODUCTION}

No single approach to the physical chemistry of soil colloids has gained acceptance in the literature. The approaches have varied from the assumption that a suspension of clay particles can be treated by the methods applicable to homogeneous solutions, at one extreme, to the concept of a soil-water mixture as a two-phase system of insoluble solid particles in an aqueous medium, at the other. In consequence, the theoretical aspects of soil chemistry are at present in a confused state.

The general basis for developing a physical chemistry for soil colloids is thermodynamics. It is a rather surprising fact that the logical structure of thermodynamics itself has not been well established. This lack is most strongly felt when efforts are made to apply thermodynamics to systems involving variables other than temperature, pressure and composition. It has therefore seemed necessary to attempt a treatment of chemical thermodynamics which has a logical form suitable for application to soil systems, and this attempt forms Section I of the present work. The method of treatment relies heavily on E. A. Guggenheim's book Thermodynamics, an Advanced Treatment for Chemists and Physicists (1949). However, Guggenheim's methods and viewpoints have been modified in many ways.
Throughout this monograph, the dogmatic position has been taken that purely thermodynamic formulas must involve only macroscopic variables of state. This classical dogma has often been contested, but the present writer feels that it must be retained if we are to preserve the great certainty which is attributed to the laws of thermodynamics. The method of attack, then, is to construct extrathermodynamic model systems and from them deduce the thermodynamic behavior of the model. Since the thermodynamics involves only observable properties, the theoretical predictions can be directly compared with experimental results.

One of the central problems in soil chemistry results from the fact that an extract must be made in order to determine the chemical characteristics of the liquid phase in a soil system or in a suspension of colloidal particles. That is, one must filter, centrifuge or dialyze the system in order to obtain an extract for chemical analysis. This means that one can only infer the state of the liquid phase in the original system on the basis of assumptions. Consequently, a large part of this monograph will be concerned with the theory of extracts. It is curious that relatively little attention, theoretical or experimental, has been given to this basic problem in soil chemistry.

\footnotetext{
${ }^{1}$ Submitted for publication August 8, 1962.
} 
The sections in which the theory of various model systems are developed are closed with a brief dicussion of their applicability to soil systems. These discussions have been intentionally kept brief, and no effort to present a literature survey has been made. Rather, in the interest of brevity, only typical papers are cited. The emphasis throughout is on the development of the theory of model systems.

Finally, the continuity of the theo- retical development of this monograph must be stressed. For example, the material on "other variables" in Section I is essential to the treatment of Donnan systems in Section II. Similarly, the Donnan theory presented in Section II is a prerequisite for the use of the doublelayer theory in Section III. The reader is accordingly urged to read the monograph as a whole, since individual sections, for the most part, will not be understood in isolation. 


\section{Section I}

\section{PERTINENT PHYSICAL CHEMISTRY}

\section{A SYSTEM OF CHEMICAL THERMODYNAMICS}

\section{Basic concepts}

In treatments of thermodynamics, one might expect to encounter first a system of well-defined concepts and then clear statements of the laws in terms of these concepts. This is not the case in most presentations. The attempt here will be to establish a system of logically consistent definitions and then to state the First and Second Laws of Thermodynamics as inductively proven generalizations in terms of these definitions.

A difficulty arises at the very start. This difficulty has been clearly stated by Kline and Koenig (1957):

The usual procedure in giving a statement of a principle is to define certain key terms which are then used in stating the principle. Usually, these key terms cannot be defined with a satisfactory degree of rigor. The whole process is then one which starts from vague qualitative notions based on physical perceptions and proceeds by a stepwise process to a condition of relative exactitude embodied in certain quantitative relations between well-defined symbols. One of the real problems in the present case is that the two key terms employed, namely, 'state' and 'property,' have been defined only very loosely, even more loosely than is usually the case. An attempt is therefore now made to improve these definitions.

The concepts of property and state are inextricably connected. Either can be defined quite easily if the other is known. There are, therefore, five possible courses of action open: (1) define either property or state and use the definition in turn to define the other; (2) assume that either one is known intuitively and use it to define the other; (3) define both together by successive stages; (4) define both at once; (5) define each in terms of the other. Of these alternatives, (4) and (5) are logically fallacious, and (3) is undesirable. In addition, there appears to be no adequate definition for either state or property alone. The only remaining course of action open, and it is not an altogether satisfactory one, is to assume that either state or property is known and then to define the other in terms of it. In this case we shall assume that we understand the term property in its general significance.

In line with this suggestion of Kline and Koenig we shall take the concept of a property of a system as a point of departure and define a subclass called primary properties as follows: A primary property is a property that has a numerical magnitude directly determinable by experimental observations carried out at some particular time. We may note, then, that the value of a primary property is independent of the history of the system, and that primary properties may be extensive (depending on the extent of the system), such as volume or mass, or intensive (independent of the extent of the system), such as pressure or density. Now, the state of a system is said to be defined when all of its primary properties have fixed values. It follows that the magnitude of the increment of a property depends only on the initial and final states of the system and not on the path of the change in state.

We may now state an important experimental generalization: It is not necessary to specify values of all primary properties in order to specify the state of a system. For example, if we fix the temperature and pressure of one mole of gaseous oxygen, all other properties of the system have determinable values and the state of the system is fixed. This leads to a generalization called the State Principle, which is stated by Kline and Koenig as follows: "For any system, there exists a positive 
integer $n$ such that if $n$ intensive primary properties are fixed, all other intensive primary properties are fixed."

Concerning the State Principle, we again quote from Kline and Koenig:

The State Principle is a fundamental postulate of thermodynamics in the same sense as are the First and Second Laws. It is not new; thermodynamicists have understood these facts for many years. However, it has been employed tacitly rather than explicitly in the vast majority of cases. No exposition of it appears to have been published previously in any form. Like the First and Second Laws of Thermodynamics, the State Principle cannot be deduced from any other fundamental macroscopic postulate, nor can it be proved by any other deductive means. Its sole proof lies in the fact that the principle and all of its deductive consequences are in accord with laboratory observations.

The existence of the State Principle means that if $p$ is any intensive primary property we can find relationships of the type

$$
p_{n+1}=f\left(p_{1}, p_{2}, \cdots p_{n}\right)
$$

Any such equation is called an equation of state, and the $p_{1} \cdots p_{n}$ are called state variables. In addition, if one extensive property is specified, the size of the system is determined.

An additional subgroup of properties may now be defined: A macroscopic property is any primary property or other properties defined in terms of primary properties.

The concept of an isolated system is fundamental to thermodynamics. By an isolated system we mean a system which is in such a condition that no change in its state can be produced by interaction with surroundings. (Such a system is contained in rigid, insulating walls in the absence of long-range forces.)

We are now in a position to deal with equilibria. Equilibrium has been defined in many ways. This is perhaps the simplest definition: Any system which is in a state of rest, that is, whose properties do not change with time, is said to be in a state of equilibrium. Equilibria may be classified as follows:

\section{Isolated systems}

A. Complete equilibrium

Every homogeneous isolated system in a state of rest is in complete equilibrium. A heterogeneous isolated system is in complete equilibrium if no processes occur in its component parts when they are brought into isolation.

B. Incomplete equilibrium

A heterogeneous isolated system in a state of rest is in incomplete equilibrium if processes occur in its component parts when they are brought into isolation.

II. Systems in contact with surroundings

A. Complete equilibrium

A system in contact with surroundings (including external force fields) is in complete equilibrium if it is in a state of rest and no processes occur when it is isolated. This is also called internal equilibrium.

B. Incomplete equilibrium

A system in contact with surroundings (including external force fields) is in incomplete equilibrium if it is in a state of rest but processes occur when it is brought into isolation. 
This classification of equilibria is essential for the form of the Second Law statement we shall adopt.

The reversible process may now be defined: Any process which occurs in a system which is only infinitesimally removed from any state of equilibrium is called a reversible process. All other processes are called spontaneous processes.

\section{Zeroth Principle}

The Zeroth Principle of Thermodynamics is so called because like the State Principle, it must logically precede the First and Second Laws. We begin by noticing that, in general, when two separate systems which are in complete equilibrium are brought into physical contact, changes occur until each attains a state of rest. In such states of rest, the two bodies are said to be in thermal equilibrium. We now follow Guggenheim's statement (1949) of the Zeroth Principle: "If two systems are in thermal equilibrium with a third system, then they are in thermal equilibrium with each other." All systems in thermal equilibrium must have a property which has the same value, namely, the temperature.

The Zeroth Principle may now be used to establish a temperature scale. Let us arbitrarily select a standard system which we will call a thermometer. We bring a thermometer into thermal equilibrium with a large reference system and find the relationship between the pressure and volume of the thermometer. The resulting pressure-volume curve must be at constant temperature and it may be called an isotherm. A temperature scale can now be defined by selecting a function of the type

$$
t=f(P, V)
$$

The only restriction on the function $f$ is that it must be consistent with the empirically found relationship between $P$ and $V$. If, for instance, the thermometer is a gas obeying Boyle's Law, we find

$$
P \cdot V=\text { constant (temperature constant) }
$$

We may then invent a temperature scale by means of any function:

$$
t=f(P V)
$$

The greatest convenience results if we use

$$
t=K(P V)
$$

where $K$ is a constant. The size of the degree is arbitrarily fixed by assigning $K=1 / n R$, where $R$ is the gas constant and $n$ is the number of moles. If, now, we wish to measure the temperature of another system, we bring it to thermal equilibrium with our thermometer, measure $P$ and $V$ of the thermometer and use the above equation to calculate $T$. We will find later that this scale has more than arbitrary significance. For the moment we note only that on this scale water freezes at $273.16^{\circ}$.

\section{First Law}

In order to state the First Law the adiabatic process must first be defined. We again follow Guggenheim and state that a process is adiabatic if the system is confined by insulating walls. Insulating walls prevent any change from occurring in a system except by movement of the wall or as the result of long-range forces such as 
external fields. We next define a function called the internal energy by means of the equation

$$
\Delta E=-w \quad \text { (adiabatic process) }
$$

where $E$ is the internal energy and $w$ is the work done by the system on the surroundings. The First Law is now stated: $E$ is a property of the system. Thus, for any change in state, $\Delta E$ is independent of process path.

Finally, for any process, we define a quantity $q$, called the heat absorbed by system, as

Thus,

$$
q=\Delta E+w
$$

$$
\Delta E=q-w
$$

is true by definition. While $\Delta E$ is independent of process path, $q$ and $w$ in general are not. Therefore, $q$ and $w$ are not properties of a system.

It is very important to note that, for the adiabatic process in which the insulating wall moves infinitesimally,

$$
d E=-P_{e x} d V \quad \text { (adiabatic process) }
$$

where $P_{e x}$ is the external pressure of the surroundings on the system, since $w=$ $P_{e x} d V$. If, but only if, the system and surroundings are infinitesimally removed from pressure equilibrium, then $P=P_{e x}$ and the reversible work is

$$
d E=-P d V \quad \text { (adiabatic process) }
$$

where $P$ is the internal pressure of the system.

\section{Enthalpy and heat capacity}

A convenient function called the enthalpy or heat content is now defined as

$$
H=E+P V
$$

Then, if the only work term is a reversible pressure-volume effect,

and

$$
d E=q-P d V
$$

Since

we find that

$$
d E=q \quad(V \text { constant })
$$

and

$$
d H=d E+P d V+V d P
$$

Thus, $d H$ is the measure of the heat effect at constant pressure.

We next define two quantities called heat capacities as

$$
\left(\frac{\partial E}{\partial T}\right)_{V}=C_{v}
$$

and

$$
\left(\frac{\partial H}{\partial T}\right)_{P}=C_{p}
$$


It follows that

$$
\begin{array}{ll}
d E=q=C_{v} d T & \\
d H=q \text { constant }) \\
=C_{p} d T &
\end{array}
$$

\section{Second Law}

In commenting on his method of treatment, Guggenheim (1949, p. 3) states,

We have deliberately chosen to regard absolute temperature and entropy, just as we regard pressure and volume, as two quantities both fundamental. We therefore do not attempt to define them in terms of other quantities regarded as simpler, for we do not admit the existence of simpler thermodynamic quantities.

This point of view is adopted here. It is furthermore felt desirable to find a statement of the Second Law which is as abstract as possible, since it is then most useful. The Second Law, then, may be stated as follows:

For any system there exists a function called the entropy $(S)$ which has the following characteristics:

(a) It is an extensive property.

(b) $d S=d_{i} S+d_{e} S$, in which $d_{i} S$ is the internal entropy change and $d_{e} S$ is the entropy change due to interactions with surroundings.

(c) $d_{e} S=q / \theta$ where $\theta$ is the absolute temperature.

(d) $d_{i} S=0$ for every reversible change.

(e) $d_{i} S>0$ for every spontaneous change.

A number of important corollaries to the Second Law can be found. We find immediately from (b) and (c) that for every change

(f) $d S=q / \theta+d_{i} S$

(every change)

From (f) and (d) we find
(g) $d S=q / \theta$
(reversible change)

From (f) and (e) we find
(h) $d S>q / \theta$
(spontaneous change)

Since $q=0$ for an isolated system, we find from (d) and (f) that
(i) $d S=0$
(reversible change, isolated system)

From (e) and (f) we find that

(j) $d S>0$

(spontaneous change, isolated system)

It is now possible to formulate equilibrium conditions. In an isolated system, $d S=0$ for every reversible process. Since every reversible process is only infinitesimally removed from a state of equilibrium,
(k) $d S=0$
(equilibrium, isolated system)

For a system in contact with surroundings, we defined complete equilibrium as a state such that it remains in equilibrium when isolated. Thus, a system is in complete equilibrium if, when it is isolated, a small displacement gives $d S=0$. 
This condition for equilibrium can be rendered more convenient by realizing that every process in isolation must be at constant $E$ and $V$. Thus, for a system in contact with surroundings a small displacement at constant $E$ and $V$ gives the same result as if the system were isolated. Therefore,

(l) $d S=0$

(E, $V$ constant; complete equilibrium)

If the system is in contact with surroundings and in incomplete equilibrium, we find from (g) that

$$
\begin{array}{ll}
\text { (m) } d S=q / \theta \quad \text { (incomplete equilibrium) }
\end{array}
$$

We notice that for a given change in state

$$
\begin{array}{ll}
q=\theta d S & \text { (reversible path) } \\
q<\theta d S & \text { (spontaneous path) }
\end{array}
$$

Since $d S$ is a property and independent of path, $q$ must be a maximum for the reversible path. Thus,
(n) $q_{\max }=\theta d S$
(reversible path)

In other treatments of the Second Law, various combinations of propositions (g) to (n) are taken as statements of the law. Here, (g) to (n) are regarded as corollaries.

Finally, for reversible processes, $q=\theta d S$ and $w=P d V+w^{\prime}$, where $w^{\prime}$ represents any additional work terms (called useful work), so that

$$
\begin{aligned}
d E & =q-w \\
& =\theta d S-P d V-w^{\prime}
\end{aligned}
$$

From the equilibrium condition (l) we know that $d E=d S=d V=0$ at complete equilibrium, so that

$$
\begin{array}{ll}
\text { (o) } w^{\prime}=0 \quad \text { (complete equilibrium) }
\end{array}
$$

Hence, useful work cannot be done on the surroundings by a system in complete equilibrium.

If a reversible process is carried on at constant $E$ and $V$ in incomplete equilibrium,

$$
\begin{aligned}
\theta d S-w^{\prime} & =0 \\
w^{\prime} & =\theta d S
\end{aligned}
$$

and useful work can be done. In fact, at constant $E, q=w$ and it follows from (n) that $w^{\prime}$ is a maximum along the reversible path.

\section{Temperature scale}

For any system containing only one component the Second Law reads

$$
d E=\theta d S-P d V
$$


By applying cross-differentiation formulas to this equation one may derive the thermodynamic equation of state:

$$
\left(\frac{\partial E}{\partial V}\right)_{\theta}=\theta\left(\frac{\partial P}{\partial \theta}\right)_{V}-P
$$

It follows that if $(\partial E / \partial V)_{\theta}=0$, then

$$
\left(\frac{\partial P}{\partial \theta}\right)_{V}=\frac{P}{\theta}
$$

This equation is satisfied only if $P$ is proportional to $\theta$ at constant $V$. However, we have previously defined a temperature scale $T$ such that, for a gas obeying Boyle's Law, $P$ is proportional to $T$. It is therefore convenient to define the perfect gas as one obeying Boyle's Law and for which $(\partial E / \partial V)_{T}=0$. Then both $T$ and $\theta$ are proportional to $P$ and therefore they are proportional to each other. By selecting the same size of degree, we obtain identical scales. The size of the degree is fixed by specifying that ice melts at $273.16^{\circ}$.

\section{Free energy}

A useful function called the Gibbs free energy $(G)$ is now defined as

$$
G=E+P V-T S
$$

From the Second Law we find that, for reversible processes,

$$
d E=T d S-P d V-w^{\prime}
$$

Differentiating equation (1-19) and substituting equation (1-20), we obtain

At constant $T$ and $P$,

$$
d G=-S d T+V d P-w^{\prime}
$$

$$
d G=-w^{\prime} \quad(T \text { and } P \text { constant })
$$

Hence, at constant $T$ and $P, d G$ is the negative of the reversible work done on the surroundings except for pressure-volume terms. Furthermore, since the complete equilibrium condition was found to be $w^{\prime}=0$, we find that

$$
d G=0 \quad(T, P \text { constant; complete equilibrium })
$$

This form of the equilibrium condition is frequently much more convenient than the one involving entropy which was stated in proposition (l).

\section{Composition variable}

Thus far we have not obtained any formulas in which the composition of the system explicitly appears as a variable. In dealing with variable composition, it is convenient to define a number of quantities such that if $X$ is any macroscopic extensive property of a system then

$$
\bar{X}_{i}=\left(\frac{\partial X}{\partial n_{i}}\right)_{T, P, n,}
$$

where $n_{i}$ is the number of moles of a given component in the system, subscript $n_{j}$ denotes all other mole numbers held constant, and $\bar{X}_{i}$ is a partial molar quantity. 
A number of relations follow from this definition. The total differential of $X$ at constant $T$ and $P$ is

$$
\begin{aligned}
d X & =\left(\frac{\partial X}{\partial n_{1}}\right)_{T, P_{i} n_{2} \ldots} d n_{1}+\cdots+\left(\frac{\partial X}{\partial n_{i}}\right)_{T, P, n_{i}} d n_{i}+\cdots \\
& =\sum_{i}\left(\frac{\partial X}{\partial n_{i}}\right)_{T, P, n_{i}} d n_{i} \\
& =\sum \bar{X}_{i} d n_{i} \quad(T, P \text { constant })
\end{aligned}
$$

This equation can be integrated by supposing that all mole numbers in the system be increased in proportion to themselves by a factor $d \alpha$ :

$$
d n_{i}=n_{i} d \alpha
$$

Since $X$ is an extensive property, it follows that

$$
d X=X d \alpha
$$

and thus equation (1-25) becomes

and

$$
X d \alpha=\sum \bar{X}_{i} n_{i} d \alpha
$$

$$
X=\sum \bar{X}_{i} n_{i}
$$

Differentiating equation (1-26) at constant $T$ and $P$, we obtain

$$
d X=\sum \bar{X}_{i} d n_{i}+\sum n_{i} d \bar{X}_{i}
$$

Comparison of this equation with equation (1-25) reveals that

$$
\sum n_{i} d \bar{X}_{i}=0 \quad(T, P \text { constant })
$$

This important result is called the Gibbs-Duhem equation. If both sides of (1-27) are divided by $\sum n_{i}$, we obtain an alternative form:

$$
\sum N_{i} d \bar{X}_{i}=0
$$

in which the equation $N_{i}=n_{i} / \sum n_{i}$ is called the mole fraction.

A useful composition variable called the "molality of a solute" is defined as the number of moles of solute per kilogram solvent. Thus, for aqueous solutions,

$$
N_{i}=\frac{m_{i}}{55.5+\sum m_{i}}
$$

where $\sum m_{i}$ includes every solute species. For the water,

$$
N_{w}=\frac{55.5}{55.5+\sum m_{i}}
$$

where subscript $w$ refers to water.

Substituting these equations into (1-28), we obtain

$$
\sum m_{i} d \bar{X}_{i}+55.5 d \bar{X}_{w}=0
$$

These equations are of great utility since, if $\bar{X}_{i}$ is known for one component of a binary mixture, they may be used to calculate $\bar{X}_{i}$ for the other. 
The Second Law is applied to systems in which the number of moles of material is variable by expressing the total differential in the internal energy as a function of $S, V$ and the $n_{i}$. Thus,

$$
d E=\left(\frac{\partial E}{\partial S}\right)_{V, n_{i}} d S+\left(\frac{\partial E}{\partial V}\right)_{S, n_{i}} d V+\sum_{i}\left(\frac{\partial E}{\partial n_{i}}\right)_{S, V, n_{i}} d n_{i}
$$

From equation (1-16) we find the first two partial coefficients (recalling that $\theta=T)$ :

$$
\begin{array}{ll}
\left(\frac{\partial E}{\partial S}\right)_{V}=T & \left(n_{i} \text { constant }\right) \\
\left(\frac{\partial E}{\partial V}\right)_{S}=-P & \left(n_{i} \text { constant }\right)
\end{array}
$$

A quantity $\mu_{i}$, called the "chemical potential," is now defined as

$$
\mu_{i}=\left(\frac{\partial E}{\partial n_{i}}\right)_{S, V, n_{i}}
$$

Equation (1-31) thus becomes

$$
d E=T d S-P d V+\sum_{i} \mu_{i} d n_{i}
$$

From (1-33) we find an expression for the free energy. First we recall that the definition of the free energy is

$$
G=E+P V-T S
$$

Differentiating this equation and substituting (1-33), we obtain

$$
d G=-S d T+V d P+\sum_{i} \mu_{i} d n_{i}
$$

Dividing (1-34) by $d n_{i}$ at constant $T, P$ and $n_{j}$ yields

$$
\left(\frac{\partial G}{\partial n_{i}}\right)_{T, P, n_{i}}=\mu_{i}=\bar{G}_{i}
$$

Thus, the partial molal free energy and the chemical potential are equal to one another.

We next find the equilibrium condition. Applying equation (1-25) to the free energy we find that

$$
d G=\sum \bar{G}_{i} d n_{i} \quad(T, P \text { constant })
$$

Furthermore, if more than one phase is present we must write

$$
d G=\sum_{\alpha} \sum_{i} \bar{G}_{i} d n_{i} \quad(T, P \text { constant })
$$

where the summation over alpha is a summation over the phases. However, the equilibrium condition has been shown to be $d G=0$ at constant $T$ and $P$. Thus,

$$
d G=\sum_{\alpha} \sum_{i} \bar{G}_{i} d n_{i}=0 \quad(T \text { and } P \text { constant; equilibrium })
$$


Now, imagine that $d n_{i}$ moles of the $i$ th component are transferred between two phases, $A$ and $B$, which are in equilibrium:

$$
d G=\bar{G}_{i}^{A} d n^{A}{ }_{i}+\bar{G}^{B}{ }_{i} d n^{B}{ }_{i}=0 \quad\left(T, P, n_{j} \text { constant }\right)
$$

But $d n^{A}{ }_{i}=-d n^{B}{ }_{i}$ and

$$
\bar{G}^{A}{ }_{i}=\bar{G}^{B}{ }_{i}
$$

We might alternatively have written

$$
\mu^{A}{ }_{i}=\mu^{B}{ }_{i}
$$

Thus it may be said that in a system at equilibrium the chemical potential of each component is constant throughout.

We next obtain an expression for the total differential in $\mu_{i}$. Choosing $T, P$ and $n_{i}$ as independent variables, we obtain

$$
d \mu_{i}=\left(\frac{\partial \mu_{i}}{\partial T}\right)_{P, n_{i}} d T^{\prime}+\left(\frac{\partial \mu_{i}}{\partial P}\right)_{T, n_{i}} d P+\left(\frac{\partial \mu_{i}}{\partial n_{i}}\right)_{T, P, n_{j}} d n_{i}
$$

The various coefficients of this expression may now be evaluated. Dividing equation (1-34) by $d T$ at constant $P$ and $n_{i}$, we find that

$$
\left(\frac{\partial G}{\partial T}\right)_{P, n_{i}}=-S
$$

Differentiating equation (1-42) with respect to $n_{i}$ at constant $T, P$ and $n_{j}$, we obtain:

$$
\frac{\partial}{\partial n_{i}}\left[\left(\frac{\partial G}{\partial T}\right)_{P, n_{i}}\right]_{P, T, n_{i}}=-\left(\frac{\partial S}{\partial n_{i}}\right)_{P, T, n_{i}}=-\bar{S}_{i}
$$

Since the order of differentiation is immaterial

and

$$
\frac{\partial}{\partial T}\left[\left(\frac{\partial G}{\partial n_{i}}\right)_{P, T, n_{i}}\right]_{P, n_{i}}=-\bar{S}_{i}
$$

$$
\left(\frac{\partial \bar{G}_{i}}{\partial T}\right)_{P, n_{i}}=-\bar{S}_{i}
$$

or

$$
\left(\frac{\partial \mu_{i}}{\partial T}\right)_{P^{\prime}, n}=-\bar{S}_{i}
$$

By an exactly analogous derivation we also find that

$$
\left(\frac{\partial \mu_{i}}{\partial P}\right)_{T, n_{i}}=\bar{\Gamma}_{i}
$$

Using $N_{i}=n_{i} /\left(n_{i}+\sum n_{j}\right)$, one may readily show that

$$
\left(\frac{\partial \mu_{i}}{\partial n_{i}}\right)_{T, P, n_{i}} d n_{i}=\left(\frac{\partial \mu_{i}}{\partial N_{i}}\right)_{T, P, n_{i}} d N_{i}
$$

Substituting equations (1-43), (1-44) and (1-45) into equation (1-41), we find that

$$
d \mu_{i}=-\bar{S}_{i} d T+\bar{V}_{i} d P+\sum\left(\frac{\partial \mu_{i}}{\partial N_{i}}\right)_{T, P, n_{i}} d N_{i}
$$


In this equation, as in equation (1-41), the summation is only over the mole fraction of chemical species whose mole number changes. The formal equations required for the solution of equilibrium conditions for cases of variable composition have now been obtained.

The treatment of variable composition is completed by finding expressions for work and heat. We have previously found that, if a process is reversible at constant $T$ and $P$, then $d G=-w^{\prime}$, where $w^{\prime}$ is the useful work. From equation (1-36) we find that

$$
\sum \bar{G}_{i} d n_{i}=-w^{\prime} \quad(T, P \text { constant; reversible process })
$$

The conclusion is that if a process involving a change in composition occurs reversibly at constant $T$ and $P$, the system will do an amount of useful work minus $\sum \bar{G}_{i} d n_{i}$ on the surroundings.

To obtain expressions for the heat effect it is convenient to write the First Law in the form

$$
d E=q-P d V-w_{e}
$$

where $w_{e}$ includes all work effects beyond a reversible pressure-volume term. ( $w_{e}$ may be different from $w^{\prime}$, since $w^{\prime}$ is always reversible work.) Differentiating the heat content equation, (1-6), and substituting equation (1-48), we find that

or

$$
d H=V d P+q-w_{e}
$$

$$
d H=q-w_{e} \quad(P \text { constant })
$$

Thus, $d H$ is not in general the measure of the heat effect. Another expression for the heat content can be found by substituting equation (1-33) for equation (1-48):

$$
d H=T d S-V d P+\sum \mu_{i} d n_{i}
$$

It should be emphasized that, while this equation is derived by considering reversible changes, it nevertheless applies to any change in state since the change in the properties of a system depend only on initial and final state. We also find that

$$
d H=T d S+\sum \mu_{i} d n_{i} \quad(P \text { constant })
$$

The heat effect for various process paths can now be found:

For a free-running chemical reaction at constant pressure, $w_{e}=0$, by definition, and

$$
d H=T d S+\sum \mu_{i} d n_{i}=q
$$

Thus, $d H$ measures the heat effect in this case.

For a reversible chemical reaction at constant pressure $w_{e}=-\sum \mu_{i} d n_{i}$, and

$$
\begin{aligned}
d H & =q+\sum \mu_{i} d n_{i} \\
q & =T d S
\end{aligned}
$$

Thus, $T d S$ measures the heat effect while $d H$ does not.

For a chemical reaction in equilibrium, $w_{e}=-\sum \mu_{i} d n_{i}=0$, and

$$
q=d H=T d S
$$




\section{SOLUTIONS}

\section{Nonelectrolytes}

In order to apply the Second Law to solutions, it is convenient to follow Lewis and Randall (1923) and define a function called the "fugacity" $\left(f_{i}\right)$ as

$$
\bar{G}_{i}=R T \ln f_{i}+B
$$

where $B$ is an arbitrary constant. We may immediately conclude that, if $B$ is chosen to have the same value everywhere, the fugacity of a component is constant throughout a system in equilibrium.

To find the significance of the fugacity, consider one component of a vapor phase which behaves as an ideal gas. Then,

$$
p_{i} \bar{V}_{i}=R T
$$

where $p_{i}$ is the partial pressure of the component. We also know that

$$
\begin{aligned}
\frac{\partial \bar{G}_{i}}{\partial p_{i}} & =\bar{V}_{i} \\
& =\frac{R T}{p_{i}}
\end{aligned}
$$

so that integration gives the equation

$$
\bar{G}_{i}=R T \ln p_{i}+c
$$

where $c$ is a constant of integration. If we make $c=B$, we find $f_{i}=p_{i}$ for every ideal vapor. In every application to be made, taking the fugacity to be equal to the vapor pressure results in a satisfactory approximation.

Raoult's Law can now be stated in the following way: In every sufficiently dilute solution, the fugacity of the solvent is proportional to its mole fraction. Thus,

$$
f_{1}=f^{\circ}{ }_{1} N_{1}
$$

where $f^{\circ}$ is the fugacity of pure solvent.

The behavior of the solute in dilute solution is found by applying the GibbsDuhem equation, (1-28):

$$
\sum N_{i} d \bar{G}_{i}=0
$$

Letting subscript 1 refer to solvent and subscript 2 refer to solute, equation (1-58) for a binary mixture becomes

$$
N_{1} d \bar{G}_{1}+N_{2} d \bar{G}_{2}=0
$$

Using equation (1-56), we find that

and

$$
\bar{G}_{1}^{\circ}=R T \ln f_{1}^{\circ}+B
$$

Therefore, in dilute solution,

$$
\bar{G}_{1}-\bar{G}_{1}^{\circ}=R^{\prime} T \ln \frac{f_{1}}{f_{1}^{\circ}}
$$

$$
\bar{G}_{1}-\bar{G}_{1}^{\circ}=R T \ln N_{1}
$$


and

$$
d \bar{G}_{1}=R T \frac{d N_{1}}{N_{1}}
$$

Using the equation $d N_{1}=-d N_{2}$ and substituting it into equation (1-59), we find that

and

$$
-R T d N_{2}+N_{2} d \bar{G}_{2}=0
$$

$$
d \bar{G}_{2}=R T \frac{d N_{2}}{N_{2}}
$$

Integrating from $N_{2}{ }^{\prime}$ to $N_{2}{ }^{\prime \prime}$ over small values of $N_{2}$ (where Raoult's Law applies), we find that

However,

$$
\bar{G}_{2}{ }^{\prime \prime}-\bar{G}_{2}{ }^{\prime}=R T \ln \frac{N_{2}{ }^{\prime \prime}}{N_{2}{ }^{\prime}}
$$

so that

$$
\bar{G}_{2}{ }^{\prime \prime}-\bar{G}_{2}^{\prime}=R T \ln \frac{f_{2}{ }^{\prime \prime}}{f_{2}{ }^{\prime}}
$$

$$
\frac{N_{2}{ }^{\prime \prime}}{N_{2}{ }^{\prime}}=\frac{f_{2}{ }^{\prime \prime}}{f_{2}{ }^{\prime}}
$$

This requires that in dilute solution the fugacity of the solute be proportional to its mole fraction:

$$
f_{2}=k N_{2}
$$

Equation (1-64) is Henry's Law and we conclude that, whenever Raoult's Law applies to the solvent, Henry's Law must apply to the solute.

A useful function called the "activity" $\left(a_{i}\right)$ is now defined as

$$
a_{i}=\frac{f_{i}}{f^{\circ}}
$$

where $f^{\circ}$ is the fugacity in an arbitrarily defined standard state. We also define an "activity coefficient" $\left(\lambda_{i}\right)$ as

$$
\lambda_{i}=\frac{a_{i}}{N_{i}}
$$

In dealing with solutes in dilute solution we see from equation (1-29) that at small values of $m_{i}$ the mole fraction and $m_{i}$ are proportional. Thus, in dilute solution,

$$
f_{i}=k^{\prime} m_{i}
$$

Correspondingly, an additional activity coefficient may be defined for solutes as

$$
\gamma_{i}=\frac{a_{i}}{m_{i}}
$$

The following characteristics of these functions may be noted immediately:

(a) The activity is always unity in the standard state.

(b) The activity is a ratio of fugacities and has no dimensions.

(c) The numerical value of $a_{i}$ at a given composition depends only on the standard state selected.

(d) Since both $a_{i}$ and $N_{i}$ have no dimensions, $\lambda_{i}$ has no dimensions.

(e) Since $a_{i}$ has no dimensions but $m_{i}$ is in moles per kilogram, $\gamma_{i}$ has the dimensions of kilograms per mole. 
The treatment is completed by selecting the standard state for the solvent by means of the condition

$$
\lim _{N_{1} \rightarrow 1} \frac{a_{1}}{N_{1}}=1
$$

Thus, $\lambda_{1} \neq 1$ is a measure of the deviation from Raoult's Law.

For the solute, a standard state is selected by either condition:

$$
\begin{aligned}
& \lim _{N_{2} \rightarrow 0} \frac{a_{2}}{N_{2}}=1 \\
& \lim _{m_{2} \rightarrow 0} \frac{a_{2}}{m_{2}}=1
\end{aligned}
$$

Thus, $\lambda_{2} \neq 1$ or $\gamma_{2} \neq 1$ is a measure of the deviation from Henry's Law. Equations (1-69) and (1-70) represent different choices of standard state and thus lead to different numerical values of the activity. At extreme dilution we find from equation (1-69) that $a_{2}=N_{2}$, while from equation (1-70) we find that $a_{2}=m_{2}$.

\section{Chemical reactions}

It is convenient to represent a generalized chemical reaction by means of the equation

$$
\sum \nu_{A} A \rightarrow \sum \nu_{B} B
$$

which signifies that various numbers of moles $\nu_{A}$ of reactants $A$ form various numbers of moles $\nu_{B}$ of products $B$. The free energy change for this process is

$$
\Delta G=\sum \nu_{B} \bar{G}_{B}-\sum \nu_{A} \bar{G}_{A}
$$

Furthermore, if all components are in their standard states,

$$
\Delta G^{\circ}=\sum \nu_{B} \bar{G}_{B}^{\circ}-\sum \nu_{A} \bar{G}_{A}^{\circ}
$$

Subtracting equation (1-72) from (1-71), we find that

$$
\Delta G-\Delta G^{\circ}=\sum \nu_{B}\left(\bar{G}_{B}-\bar{G}_{B}^{\circ}\right)-\sum \nu_{A}\left(\bar{G}_{A}-\bar{G}_{A}^{\circ}\right)
$$

However, from equations (1-56) and (1-65), we find that

so that

$$
\bar{G}_{i}-\bar{G}_{i}^{\circ}=R T \ln a_{i}
$$

$$
\Delta G-\Delta G^{\circ}=\sum \nu_{B} R T \ln a_{B}-\sum \nu_{A} R T \ln a_{A}
$$

This equation may be rewritten in the form

$$
\Delta G-\Delta G^{\circ}=R T \ln \frac{\Pi\left(a_{B}\right)^{\nu_{B}}}{\Pi\left(a_{A}\right)^{\nu_{A}}}
$$

where $\Pi$ denotes the product. Recalling that $\Delta G=0$ is the condition for equilibrium, we find that

$$
-\Delta G^{\circ}=R T \ln \frac{\Pi\left(a_{B}\right)^{\nu_{B}}}{\Pi\left(a_{A}\right)^{\nu_{A}}} \quad \text { (equilibrium) }
$$

Since $\Delta G^{\circ}$ is independent of composition we may write

in which

$$
\frac{\Pi\left(a_{B}\right)^{\nu_{B}}}{\Pi\left(a_{A}\right)^{\nu_{A}}}=k
$$

$$
-\Delta G^{\circ}=R T \ln k
$$

and $k$ is the equilibrium constant of the reaction. 


\section{Solutions of strong electrolytes}

The treatment of strong electrolyte solutions is begun by assuming that in very dilute solution, Henry's Law applies to individual ionic species. We therefore select the standard state for the individual ions by means of the condition

$$
\lim _{m_{i} \rightarrow 0} \frac{a_{i}}{m_{i}}=1
$$

An ionic activity coefficient is also defined as

$$
\gamma_{i}=\frac{a_{i}}{m_{i}}
$$

so that $\gamma_{i}=1$ at infinite dilution.

We next select a standard state for the electrolyte species which is consistent with this choice for ions. If an electrolyte $X$ dissociates into $\nu_{+}$cations and $\nu_{-}$ anions, the process may be represented by writing

$$
X=\nu_{+} X_{+}+\nu_{-} X_{-}
$$

We have already defined the standard states of the ions so that the standard state for the electrolyte species may be chosen according to the formula:

$$
\bar{G}_{X}^{\circ}=\nu_{+} \bar{G}_{X+}^{\circ}+\nu_{-} \bar{G}^{\circ}{ }_{X-}
$$

This selection results in the equation

$$
\Delta G^{\circ}=0
$$

Thus from equation (1-79) we find that

$$
k=1
$$

and from equation (1-78) we find that

$$
\frac{\left(a_{+}\right)^{\nu_{+}} \cdot\left(a_{-}\right)^{\nu_{-}}}{a_{X}}=1
$$

It is next convenient to define the mean activity, mean activity coefficient, and mean molality of the electrolyte as

$$
\begin{aligned}
a_{ \pm} & =\left(a_{+}{ }^{\nu_{+}} \cdot a_{-}{ }^{\nu_{-}}\right)^{1 / \nu} \\
\gamma_{ \pm} & =\left(\gamma_{+}{ }^{\nu_{+}} \cdot \gamma_{-}{ }^{\nu_{-}}\right)^{1 / \nu} \\
m_{ \pm} & =\left(m_{+}{ }^{\nu_{+}} \cdot m_{-}{ }^{\nu_{-}}\right)^{1 / \nu}
\end{aligned}
$$

where $\nu=\nu_{+}+\nu_{-}$. Dividing equation (1-85) by equation (1-87), we obtain

or

$$
\begin{aligned}
& \frac{a_{ \pm}}{m_{ \pm}}=\left[\left(\frac{a_{+}}{m_{+}}\right)^{\nu+} \cdot\left(\frac{a_{-}}{m_{-}}\right)^{\nu-}\right]^{1 / \nu} \\
& =\left[\gamma_{+}{ }^{\nu+} \cdot \gamma_{-}{ }^{\nu}\right]^{1 / \nu} \\
& =\gamma_{ \pm}
\end{aligned}
$$

$$
a_{ \pm}=\gamma_{ \pm} m_{ \pm}
$$


From equation (1-86) it is evident that since $\gamma_{+}$and $\gamma_{-}$go to unity at infinite dilution, $\gamma_{ \pm}$also goes to unity at infinite dilution. ${ }^{2}$

In all purely thermodynamic formulations, it is the activity of the electrolyte $a_{x}$ which appears, or combinations of ionic activities which reduce to $a_{x}$, since ionic activities cannot be measured. The value of $a_{x}$ can be found either from $a_{ \pm}$or from $\gamma_{ \pm}$and the molalities by means of the formulas

$$
\begin{aligned}
& a_{x}=\left(a_{ \pm}\right)^{\nu} \\
& a_{x}=\gamma_{ \pm}^{\nu} \cdot m_{+}{ }^{\nu_{+}} \cdot m_{-}^{{ }^{\nu-}}
\end{aligned}
$$

\section{Osmotic pressure}

It seems desirable to include here a treatment of the osmotic pressure of aqueous solutions, since it is a quantity which has found widespread application to soil solutions. Furthermore, many textbooks have presented erroneous derivations of the osmotic pressure equations.

Consider a system composed of two aqueous phases contained in an apparatus which permits the pressure on either phase to be varied by means of pistons. Phase I consists of a solution of nonelectrolyte and phase II contains pure water. The phases are separated by a membrane, $M$, permeable to water but not to solute.

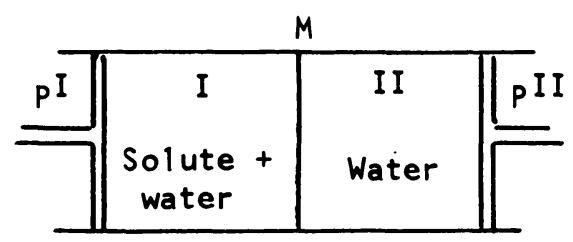

In general, water will tend to diffuse across the membrane when $P^{\mathrm{I}}=P^{\mathrm{II}}$. However, this diffusion can be prevented by increasing the pressure on phase I until $\bar{G}$ for the water is the same in the two phases. Thus, at equilibrium

$$
\bar{G}_{w}^{\mathrm{I}}=\bar{G}_{w} \mathrm{II}
$$

where subscript $w$ stands for water. The pressure required to establish equilibrium is readily found from equation (1-44):

$$
\frac{\partial \bar{G}_{w}}{\partial P}=\bar{V}_{w}
$$

Letting $P^{\text {II }}=P^{\circ}$ be the standard pressure, we integrate the pressure of phase I from $P^{\circ}$, where $\bar{G}_{w}=\bar{G}_{w}{ }^{\mathrm{I}}$, up to $P_{\text {eq }}{ }^{\mathrm{I}}$, where $\bar{G}_{w}=\bar{G}^{\circ}{ }_{w}$. Thus,

$$
\int_{\bar{G}_{w} \mathrm{I}}^{\bar{G}^{\circ}{ }_{w}} d \bar{G}_{w}=\int_{P^{\circ}}^{P_{\mathrm{eq}} \mathrm{I}} \bar{V}_{w} d P
$$

Assuming $\bar{V}_{w}$ to be constant,

$$
\bar{G}_{w}{ }^{\mathrm{I}}-\bar{G}_{w}^{\circ}=-\bar{V}_{w}\left(P_{\mathrm{eq}}^{\mathrm{I}}-P^{\circ}\right)
$$

${ }^{2}$ It should be emphasized that we have not chosen infinite dilution as the standard state, but only as a reference state in which $\gamma_{\dot{ \pm}}$ is unity. In the standard state, $a_{ \pm}$is equal to unity. 
Therefore, if $a_{w}{ }^{\mathrm{I}}$ is the activity of the water at standard pressure $P^{\circ}$,

$$
P_{\text {eq }}{ }^{\mathrm{I}}-P^{c}=-\frac{R T}{\bar{V}_{w}} \ln a_{w}{ }^{\mathrm{I}}
$$

The osmotic pressure (I) is now defined as

$$
\Pi=P_{\mathrm{eq}}^{\mathrm{I}}-P^{\circ}
$$

The osmotic pressure is thus defined as the pressure that must be applied to a solution to equilibrate the solution with pure water. Then,

$$
\Pi=-\frac{R T}{\bar{V}_{w}} \ln a_{w}{ }^{\mathrm{I}}
$$

For dilute solutions of nonelectrolytes, many approximate forms of equation (1-97) may be derived. We may first obtain $\Pi$ as a function of the solute concentration by applying the Gibbs-Duhem equation in the form of equation (1-30):

$$
55.5 d \mu_{w}+m_{S} d \mu_{S}=0
$$

in which subscript $S$ stands for solute. Therefore,

$$
\begin{gathered}
55.5 d \ln a_{w}+m_{S} d \ln a_{S}=0 \\
\ln a_{w}=-\int_{0}^{m_{S}} \frac{m_{S} d \ln a_{S}}{55.5}
\end{gathered}
$$

Substitution of equation (1-100) into equation (1-97) gives

$$
\Pi=\frac{R T}{55.5 \bar{V}_{w}} \int_{0}^{m_{S}} m_{s} d \ln a_{S}
$$

For dilute solutions, $a_{S}=m_{S}$ and $55.5 \bar{V}_{w}=1$, so that

$$
\Pi=m_{S} R T
$$

The similarity between equation (1-102) and the ideal gas law is wholly without significance.

An entirely analogous derivation for solutions involving strong electrolytes is possible except that the Gibbs-Duhem equation must be written

$$
55.5 d \mu_{w}+\sum m_{i} d \mu_{i}=0
$$

where the summation is over every individual ionic species present. We obtain

$$
\Pi=\frac{R T}{55.5 \bar{V}_{w}} \int_{0}^{m_{i}} \sum m_{i} d \ln a_{i}
$$

In extremely dilute solution this becomes

$$
\Pi=R T \sum m_{i}
$$

\section{Principle of ionic strength}

The data available for $\gamma_{ \pm}$in mixtures of electrolytes are limited. This lack would constitute a serious problem in dealing with soil solutions if Lewis and Randall 
(1923) had not discovered the principle of ionic strength. To state this principle a new quantity called the "ionic strength" $(s)$ is defined as

$$
s=\frac{1}{2} \sum z^{2} m_{i}
$$

where $z_{i}$ is the valence. Thus, in a mixture of electrolytes, the ionic strength is found by multiplying the square of the valence and molality, summing over every ionic species present, and dividing the result by 2 . The principle of ionic strength then states: In dilute solutions, the mean activity coefficient of a given strong electrolyte is the same in all solutions of the same ionic strength.

This principle is of great use in dilute mixtures such as soil solutions since the mean activity coefficient of an electrolyte can be estimated from data obtained on a single-salt solution. For example, assume that $\gamma_{ \pm\left(\mathrm{CaCl}_{2}\right)}$ data are available as a function of molality $(m)$ of the $\mathrm{CaCl}_{2}$. The ionic strength is

$$
s=\frac{1}{2}[4 m+2 m]=3 m
$$

Thus, if we have a mixture of ionic strength $(s)$ containing $\mathrm{CaCl}_{2}$, then $\gamma_{ \pm}\left(\mathrm{CaCl}_{2}\right)$ in this mixture may be estimated by finding $\gamma_{ \pm}$in pure $\mathrm{CaCl}_{2}$ at molality $s / 3$.

\section{Debye-Hückel theory}

The Debye-Hückel theory will be presented here in some detail since it has much formal similarity to the double-layer theory for colloids. Moreover, very similar theories have been used to estimate ionic interactions in suspensions. This presentation will not be rigorous, but will emphasize those aspects of the theory most relevant for later work. (For a more detailed treatment, see Harned and Owen, 1958.)

The concern of the Debye-Hückel theory $(\mathrm{DH})$ is to calculate the activity coefficient of an ionic species in dilute solution. The first step is to write the relationship between the ionic activity and the chemical potential:

$$
\mu_{i}-\mu_{i}^{\circ}=R T \ln a_{i}
$$

Further, the ionic activity coefficient has been so defined that it measures the deviations from Henry's Law with the result that

$$
\begin{aligned}
\mu_{i}-\mu_{i}^{\circ} & =R T \ln \gamma_{i} m_{i} \\
& =R T \ln \gamma_{i}+R T \ln m_{i}
\end{aligned}
$$

That is to say, where Henry's Law applies to the ions we find $\gamma_{i}=1$, and

$$
\mu_{i}-\mu_{i}^{\circ}=R T \ln m_{i}
$$

In the DH theory it is assumed that all deviations from Henry's Law are caused by electrostatic interactions between the ions. Thus, if $\mu_{i}(e l)$ is that part of the chemical potential which is due to electrostatic interactions we may write

$$
\mu_{i}(e l)=R T \ln \gamma_{i}
$$

The problem then becomes to calculate $\mu_{i}(e l)$.

A basic point of departure in the Debye-Hückel theory is the concept of the ion atmosphere. We imagine that a given ion in the solution can be singled out and labeled the "central ion." The other ions which surround this central ion consti- 
tute what is called the "ion atmosphere." The Poisson equation is then applied to this assemblage of the central ion and its atmosphere. This equation reads,

$$
\nabla^{2} \phi=-\frac{4 \pi \rho}{D}
$$

in which $\phi$ is the electric potential, $\rho$ is the charge density of a volume element of solution, and $D$ is the dielectric constant. It may be remarked in passing that the Poisson equation is derived from the classical theory of electrostatics in which the charge density may vary continuously. Thus, in the mere assumption that Poisson's equation can be applied, it has been assumed that the ions are point charges.

In spherical coordinates, equation (1-111) becomes

$$
\frac{1}{r^{2}} \frac{\partial}{\partial r}\left(r^{2} \frac{\partial \phi}{\partial r}\right)=-\frac{4 \pi \rho}{D}
$$

Now, to apply this equation to the central ion and its atmosphere, it is assumed that $r$ is the distance measured outward from the central ion and that the distribution of the ion atmosphere is spherically symmetrical about the central ion. This permits the removal of the partial signs:

$$
\frac{1}{r^{2}} \frac{d}{d r}\left(r^{2} \frac{d \phi}{d r}\right)=-\frac{4 \pi \rho}{D}
$$

It is next assumed that the concentrations of the ions in the atmosphere will be determined by the Boltzmann equation in the form,

$$
n_{i}=n^{\circ}{ }_{i} \exp \left(-\frac{z_{i} \ell \phi}{k T}\right)
$$

in which $n_{i}$ is the ion concentration in ions per cc, $n^{\circ}{ }_{i}$ is the bulk ion concentration in the solution, $z_{i}$ is the valence, $e$ the charge on the electron, $k$ the Boltzmann constant, $T$ is the absolute temperature, and $\phi$ is again the electric potential. An analysis of the assumptions underlying the applicability of the Boltzmann equation would carry the present discussion too far afield. (See Bockris, 1959, Chapter I.)

The density of charge at any distance $r$ from the central ion will simply be equal to the difference between the concentration of negative and positive charges:

$$
\rho=\sum z_{i} e n_{i}
$$

Combining equations (1-113), (1-114) and (1-115), we obtain:

$$
\frac{1}{r^{2}} \frac{d}{d r}\left(r^{2} \frac{d \phi}{d r}\right)=-\frac{4 \pi}{D} \sum z_{i} e n_{i}^{\circ} \exp \left(-\frac{z_{i} \ell \phi}{k T}\right)
$$

We now have a differential equation in $\phi$ and $r$, but no general solution exists. To obtain a solution, the factor $\exp \left[-\left(z_{i} e \phi\right) / k T\right]$ must be expressed in a series:

$$
\exp \left(-\frac{z_{i} \ell \phi}{k T}\right)=1-\frac{z_{i} \ell \phi}{k T}+\frac{1}{2 !}\left(\frac{z_{i} \ell \phi}{k T}\right)^{2}-\cdots
$$

In very dilute solution, $\left(z_{i} e \phi\right) / k T \ll 1$, so that

$$
\exp \left(-\frac{z_{i} \ell \phi}{k T}\right) \cong 1-\frac{z_{i} \ell \phi}{k T}
$$


Then,

$$
\begin{aligned}
\frac{1}{r^{2}} \frac{d}{d r}\left(r^{2} \frac{d \phi}{d r}\right) & =-\frac{4 \pi}{D} \sum z_{i} e n_{i}{ }_{i}\left[1-\frac{z_{i} \ell \phi}{k T}\right] \\
& =-\frac{4 \pi}{D} \sum z_{i} e n_{i}{ }_{i}+\frac{4 \pi}{D} \sum \frac{z^{2} e^{2} n^{\circ}{ }_{i \phi}}{k T}
\end{aligned}
$$

The first term on the right-hand side must be zero owing to the overall electrostatic neutrality of the solution. Therefore,

$$
\frac{1}{r^{2}} \frac{d}{d r}\left(r^{2} \frac{d \phi}{d r}\right)=\frac{4 \pi}{D} \sum \frac{z^{2} e^{2} n^{\circ}{ }_{i} \phi}{k T}
$$

For convenience we define

so that

$$
\kappa^{2}=\frac{4 \pi}{D} \sum \frac{z^{2} i^{2} n^{\circ}}{k T}
$$

$$
\frac{1}{r^{2}} \frac{d}{d r}\left(r^{2} \frac{d \phi}{d r}\right)=\kappa^{2} \phi
$$

This equation readily integrates into

$$
\phi=\frac{A}{r} \exp (-\kappa r)+\frac{B}{r} \exp (\kappa r)
$$

in which $A$ and $B$ are constants of integration. From the boundary condition that $\phi=0$ at $r=\infty$, we immediately find that $B=0$.

To evaluate $A$, we notice that since $\kappa$ contains $n^{\circ}{ }_{i}, \kappa$ must go to zero as $n^{\circ}{ }_{i}$ goes to zero. Further, when $n^{\circ}{ }_{i}$ is zero, the ion atmosphere has vanished so that only the central ion is left. Under these conditions, the potential equation must reduce to the potential distribution around a single point charge. Thus, at $\kappa=0$,

Therefore,

$$
\phi=\frac{A}{r}=\frac{z_{i} e}{D r}
$$

and

$$
A=\frac{z_{i} e}{D}
$$

$$
\phi=\frac{z_{i} e}{D r} \exp (-\kappa r)
$$

The term exp $(-\kappa r)$ is also expanded according to

Therefore,

$$
\exp (-\kappa r) \cong 1-\kappa r
$$

$$
\begin{aligned}
\phi & =\frac{z_{i} e}{D r}[1-\kappa r] \\
& =\frac{z_{i} e}{D r}-\frac{z_{i} e \kappa}{D}
\end{aligned}
$$

An inspection of this equation reveals that the first term is that part of the potential which is due to the central ion only, and it is independent of concentration. Thus, the second term must represent the potential due to the ion atmosphere which is 
dependent on concentration but independent of distance. Thus, if $\phi^{*}$ is the potential due to the atmosphere, we find that

$$
\phi^{*}=-\frac{z_{i} \kappa}{D}
$$

The remainder of the development of the $\mathrm{DH}$ theory is concerned with $\phi^{*}$ only. The reason is that we are interested only in that part of the electrical potential which produces deviations from Henry's Law, and only ionic interactions produce such deviations. If the ions do not interact, they would obey Henry's Law even though charged. This is indeed the situation at infinite dilution.

At the present point, the electric potential due to the ion atmosphere, or due to ionic interactions, has been found. We must next find $\mu_{i}(e l)$. This is done by calculating the reversible work necessary to increase the charge on the ions in the atmosphere from an initial value of zero up to their full value of $z_{i} e$. This work gives the extra potential energy of the system due to ion interactions and this extra potential energy is just $\mu_{i}(e l)$. The reversible work required to change the charge on a point electrostatic charge is expressed:

Thus, for one mole of ions,

$$
\text { work }=\phi d q
$$

$$
\mu_{i}(e l)=N_{A} \int_{0}^{z_{i} e} \phi^{*} d\left(z_{i} e\right)
$$

where $N_{A}$ is the Avagadro number. Substituting equation (1-126) and integrating, we find that

$$
\mu_{i}(e \tilde{l})=-\frac{z^{2}{ }_{i} e^{2} N_{A} \kappa}{2 D}
$$

Substituting equation (1-128) into equation (1-110), we find that for the activity coefficient

$$
R T \ln \gamma_{i}=-\frac{z^{2}{ }_{i} e^{2} N_{A} \kappa}{2 D}
$$

From equation $(1-120)$ note that $\kappa^{2}$ is a function of $\sum z^{2} n^{\circ}{ }_{i}$. Since the ionic strength is given by $s=\frac{1}{2} \sum z^{2} m_{i}$, it follows that equation (1-129) can be written as a function of $s^{1 / 2}$. The result is

in which

$$
\log _{10} \gamma_{i}=-z^{2} S s^{1 / 2}
$$

$$
S=\frac{N^{2}{ }_{A} e^{3}}{2.3(D R T)^{3 / 2}} \cdot\left(\frac{2 \pi \rho}{1,000}\right)^{1 / 2}
$$

where $\rho$ is the density of the solution. At $25^{\circ} \mathrm{C}, S=0.511$. This is the final $\mathrm{DH}$ equation for ionic activity coefficient.

By noting that for any electrolyte, $\nu_{+} z_{+}=\nu_{-}\left|z_{-}\right|$and combining equation (1-86) with equation (1-130), we find the mean activity coefficient for an electrolyte:

$$
\log _{10} \gamma_{ \pm}=-\left|z_{+} \cdot z_{-}\right| S s^{1 / 2}
$$

At this point, two inconsistencies in the theory should be noted. According to equation (1-125) the electrical potential $(\phi)$ rises to infinity at $r=0$. Yet, we have assumed $z_{i} e \phi \ll k T$. (This difficulty does not arise with $\phi^{*}$, which is independent 
of distance.) Further, we evaluate the integration constant $B$ by making $\phi=0$ at $r=\infty$. Yet, in equation (1-124) it was assumed that $\kappa r \ll 1$. The answer to these difficulties appears to be simply that the numerical error introduced is not large. For a full discussion, see Alexander and Johnson (1949).

There have been many efforts to extend the DH theory to higher concentrations. Only one will be presented here. It involves the introduction of "the distance of closest approach" of the ions. Without presenting the details, what is involved is a reevaluation of the constant of integration $A$ when the ions have a minimum distance of approach $a_{i}$. Under these conditions $A$ becomes

$$
A=\frac{z_{i} e}{D}\left[\frac{\exp \left(\kappa a_{i}\right)}{1+\kappa a_{i}}\right]
$$

Using equation (1-122), with $B=0$, we find that

$$
\phi=\frac{z_{i} e}{D r}\left[\frac{\exp \kappa\left(a_{i}-r\right)}{1+\kappa a_{i}}\right]
$$

At the minimum distance $r=a_{i}$, and

$$
\phi\left(a_{i}\right)=\frac{z_{i} e}{D a_{i}\left(1+\kappa a_{i}\right)}
$$

The expression on the right may be separated into two terms:

$$
\phi\left(a_{i}\right)=\frac{z_{i} e}{D a_{i}}-\frac{z_{i} e \kappa}{D\left(1+\kappa a_{i}\right)}
$$

The first term is the potential due to the central ion, so that

$$
\phi^{*}\left(a_{i}\right)=-\frac{z_{i} e \kappa}{D\left(1+\kappa a_{i}\right)}
$$

Integrating to find $\mu_{i}(e l)$ and solving for the activity coefficient, we find that

$$
\log _{10} \gamma_{i}=-\frac{z_{i} S s^{1 / 2}}{1+\beta a_{i} s^{1 / 2}}
$$

where $\beta$ is a constant equal to $3.286 \times 10^{-9}$, and $a_{i}$ is the distance of closest approach in $\AA$. Note that in this derivation, the expansion of exp $(-\kappa r)$ is avoided.

The DH theory, as a limiting law, has found widespread experimental verification. It not only predicts the principle of ionic strength, but gives the correct numerical magnitude of the limiting slopes of activity coefficient curves for a wide variety of valence types. However, as might be expected from the assumptions of the theory, it applies only to extremely dilute solutions. At higher concentrations, it provides a basis for the evaluation of other types of deviations from Henry's Law.

\section{SYSTEMS INVOLVING OTHER VARIABLES}

\section{General considerations}

Thermodynamic equations for systems involving variables other than $T, P$, and $n_{i}$ are available in many places. However, the method of treatment is not always the same. In particular, the use of the chemical potential has varied. It will be important to examine systems involving other variables closely, since soil systems also involve additional variables. Further, the macroscopic properties of systems 
in force fields, such as electrostatic fields, are of interest since they frequently form the microscopic properties of matter in a soil system.

Consider the case in which a complete specification of the energy change for a certain process requires the specification of not only the changes in $S, V$ and $n_{i}$, but also the changes in the extensive variables $x_{1}, x_{2} \cdots x_{p} \cdots$. In the same manner that the $n_{i}$ were introduced, we write

By defining

$$
d E=T d S-P d V+\sum \mu_{i} d n_{i}+\sum\left(\frac{\partial E}{\partial x_{p}}\right)_{S, V, n_{i}, x_{o}} d x_{p}
$$

$$
X_{p}=\left(\frac{\partial E}{\partial x_{p}}\right)_{S, V, n_{i}, x_{q}}
$$

and substituting equation (1-140) in equation (1-139), we find that

$$
d E=T d S-P d V+\sum \mu_{i} d n_{i}+\sum X_{p} d x_{p}
$$

This equation leads at once to:

$$
\left(\frac{\partial E}{\partial n_{i}}\right)_{S, V, n_{j}, x_{p}}=\mu_{i}
$$

It is with equation (1-142) that difficulties enter. In certain cases, we require the specification of the $x_{p}$ variable, but its value is not independent of one of the other extensive variables in equation (1-141). We will later encounter two examples of the sort. In the case of gravitational fields, we will find that

$$
\begin{aligned}
X_{p} & =g h \\
d x_{p}=d m & =\sum M_{i} d n_{i}
\end{aligned}
$$

in which $g=$ acceleration of gravity, $h=$ height, $m=$ mass, and $M_{i}$ is molecular weight. In the electrochemical case, we find that

$$
\begin{gathered}
X_{p}=\psi \\
d x_{p}=d q=\sum z_{i} F_{y} d n_{i}
\end{gathered}
$$

in which $\psi=$ electric potential, $q=$ charge, and $F_{y}$ is the Faraday constant.

In both of these cases, specification of the $x_{p}$ variable is required but it is not independent of $n_{i}$. Thus, equation (1-142) for the chemical potential does not exist. Rather, the substitution of the equations for $X_{p}$ and $x_{p}$ in equation (1-141) yields

and

$$
d E=T d S-P d V+\sum\left(\mu_{i}+M_{i} g h\right) d n_{i}
$$

$$
d E=T d S-P d V+\sum\left(\mu_{i}+z_{i} F_{y} \psi\right) d n_{i}
$$

These equations lead to:

and

$$
\left(\frac{\partial E}{\partial n_{i}}\right)_{S, V, n_{i}}=\mu_{i}+M_{i} g h
$$

$$
\left(\frac{\partial E}{\partial n_{i}}\right)_{S, V, n_{j}}=\mu_{i}+z_{i} F_{y} \psi
$$


In other instances, the $x_{p}$ variable is independent of any of the other extensive variables in equation (1-141). In the case of electrostatic fields, we find that

$$
\begin{gathered}
X_{p}=\mathcal{E} \\
d x_{p}=d \mathscr{P}
\end{gathered}
$$

in which $\mathcal{E}=$ field strength and $\mathcal{P}=$ polarization.

In the case of magnetic fields, we find that

$$
\begin{aligned}
X_{p} & =\mathfrak{H C} \\
d x_{p} & =d I
\end{aligned}
$$

in which $\mathfrak{F}$ is the field strength and $I$ is the magnetic intensity. Since $\mathscr{P}$ and $I$ are independent variables, we may apply equation (1-142) without difficulty:

$$
\begin{aligned}
& \left(\frac{\partial E}{\partial n_{i}}\right)_{S, V, n_{j}, \beta}=\mu_{i} \\
& \left(\frac{\partial E}{\partial n_{i}}\right)_{S, V, n_{i}, I}=\mu_{i}
\end{aligned}
$$

It is evident that the thermodynamic treatment of other variables must depend upon the experimentally known characteristics of the variables. The significance of $\mu_{i}$ in equations (1-147) and (1-148) differs from its significance in equations (1-155) and (1-156), but a point worthy of considerable emphasis is that the significance of $\mu_{i}$ is not arbitrary once we have written equation (1-141).

\section{Gravitational fields}

If a piece of matter is present in a gravitational field, it is known from mechanics that it has a potential energy, $E_{g}$, given as

$$
E_{g}=m \phi
$$

where $m=$ mass and $\phi=g h$, the gravitational potential. By the method used above this leads at once to the formula:

and

$$
d E=T d S-P d V+\sum\left(\mu_{i}+M_{i} \phi\right) d n_{i}
$$

$$
\left(\frac{\partial E}{\partial n_{i}}\right)_{S, V, n_{i}}=\mu_{i}+M_{i} \phi
$$

We now ask how an equation for the chemical potential can be found. In the present case this presents no problem since it is a characteristic of the gravitational field that the potential is independent of the presence of matter, so that $\phi$ is determinable by direct measurements of space (that is, $h$ ). Further, it is a characteristic of matter that its intensive primary properties are not affected by the presence of a gravitational field. Thus, all the formulas developed for $\mu_{i}$ in the absence of the field are valid in its presence. Therefore, we may write

$$
\left(\frac{\partial E}{\partial n_{i}}\right)_{S, V, n_{j}(\phi=0)}=\mu_{i}
$$

in the presence of the field, provided its value is constant. 
The formula for the free energy may now be found. Resorting to the definition of $G$, we know that

$$
G=E+P V-T S
$$

Differentiating this equation and substituting equation (1-158) for $d E$, we find for the total differential in $G$ :

$$
d G=-S d T+V d P+\sum\left(\mu_{i}+M_{i} \phi\right) d n_{i}
$$

Dividing by $d n_{i}$ at constant $T, P$, and $n_{j}$, we find that

$$
\left(\frac{\partial G}{\partial n_{i}}\right)_{T, P, n_{i}}=\bar{G}_{i}=\mu_{i}+M_{i} \phi
$$

Thus, $\bar{G}_{i}$ is no longer equal to $\mu_{i}$ when $\phi \neq 0$.

The condition for complete equilibrium expressed in equation (1-23) becomes

$$
d G=\sum\left(\mu_{i}+M_{i} \phi\right) d n_{i}=0 \quad(T, P \text { constant })
$$

For matter distributed between more than one phase we find that, analogous to equation (1-38),

$$
d G=\sum_{\alpha} \sum_{i}\left(\mu_{i}+M_{i} \phi\right) d n_{i}=0 \quad(T, P \text { constant })
$$

For the transfer of $d n_{i}$ moles between two phases, $A$ and $B$, equation (1-164) becomes

$$
\left(\mu_{i}+M_{i} \phi\right)^{A} d n_{A}+\left(\mu_{i}+M_{i} \phi\right)^{B} d n_{B}=0 \quad\left(T, P, n_{j} \text { constant }\right)
$$

Since $d n_{A}=-d n_{B}$, we find that

$$
\left(\mu_{i}+M_{i} \phi\right)^{A}=\left(\mu_{i}+M_{i} \phi\right)^{B}
$$

Alternatively, this equilibrium condition can be written in the form,

$$
d \bar{G}_{i}=d \mu_{i}+M_{i} d \phi=0
$$

The equilibrium condition given in equation (1-167) can be used to derive the pressure gradient in a fluid in a gravitational field. Retaining the same formula for $d \mu_{i}$ as on the absence of the field, we have for a single component $d \mu_{i}=\bar{V}_{i} d P$, and using $d \phi=g d h$, equation (1-167) becomes

$$
\begin{aligned}
\bar{V}_{i} d P+M_{i} g d h & =0 \quad(T \text { constant }) \\
\frac{d P}{d h} & =-\frac{M_{i}}{\bar{V}_{i}} g \\
& =-\rho g
\end{aligned}
$$

This is the familiar formula for hydrostatic equilibrium.

If a binary mixture is present in a gravitational field at constant temperature, we substitute equation (1-46) in equation (1-167) as in the absence of the field and obtain, at constant $T$,

$$
\bar{V}_{i} d P+\left(\frac{\partial \mu_{i}}{\partial N_{i}}\right)_{T, P, n_{i}} d N_{i}+M_{i} g d h=0
$$


Solving for $d N_{i} / d h$, we find that

$$
\begin{aligned}
\frac{d N_{i}}{d h} & =-\frac{M_{i} g+\bar{V}_{i}\left(\frac{d P}{d h}\right)}{\left(\frac{\partial \mu_{i}}{\partial N_{i}}\right)_{T, P, n_{i}}} \\
& =-\frac{M_{i} g-\bar{V}_{i} \rho g}{\left(\frac{\partial \mu_{i}}{\partial N_{i}}\right)_{T, P, n_{j}}}
\end{aligned}
$$

in which $\rho$ is the density of the solution.

Finally, we may confirm that chemical equilibria are independent of the field. Applying previous nomenclature, a chemical reaction is expressed as

$$
\sum \nu_{A} A \rightarrow \sum \nu_{B} B
$$

The equilibrium condition is

or

$$
\begin{gathered}
\Delta G=\sum \nu_{B} \bar{G}_{B}-\sum \nu_{A} \bar{G}_{A}=0 \\
\sum \nu_{B}\left(\mu_{B}+M_{B} \phi\right)=\sum \nu_{A}\left(\mu_{A}+M_{A} \phi\right)
\end{gathered}
$$

However, owing to conservation of mass,

$$
\sum \nu_{B} M_{B}=\sum \nu_{A} M_{A}
$$

Thus from equation (1-173) we obtain the formula

$$
\sum \nu_{B} \mu_{B}=\sum \nu_{A} \mu_{A}
$$

just as in the absence of the field.

\section{Electrostatic fields}

For simplicity, we will develop the formula for a system which consists of a parallel plate condensor, with unit capacity in vacuo, immersed in a dielectric medium. In this case we have $x_{p}=q$ where $q$ is the charge on the condensor and $X_{P}=\Delta \psi$, the electric potential difference. Therefore,

$$
d E=T d S-P d V+\sum \mu_{i} d n_{i}+\Delta \psi d q
$$

In this instance, $q$ is an independent variable so that to unambiguously define $\mu_{i}$, we must write

$$
\left(\frac{\partial E}{\partial n_{i}}\right)_{S, V, n_{j}, q}=\mu_{i}
$$

Using equation (1-176), together with the definition of $G$, the total differential in $G$ is found:

$$
d G=-S d T+V d P+\sum \mu_{i} d n_{i}+\Delta \psi d q
$$

Dividing by $d n_{i}$ at constant $T, P, n_{j}$ and $q$,

$$
\left(\frac{\partial G}{\partial n_{i}}\right)_{T, P, n_{j}, q}=\bar{G}_{i}=\mu_{i}
$$

Thus, in contrast to the former case, $\bar{G}_{i}$ is equal to $\mu_{i}$. However, unlike the gravitational case, the intensive properties of the material between the plates depends on the field, and further, the field strength between the plates depends on the presence or absence of matter. 
As the result of the lack of any direct knowledge of the field strength between the plates, we are also in doubt about the pressure. We follow Guggenheim's treatment (1949), and define $P$ as the pressure outside the plates. It must also be recognized that $V$ is the volume of the whole system.

As the result of the effect of the field on the intensive properties, we must now include an extra term in the expression for the total differential in $\mu_{i}$. Thus, equation $(1-46)$ is replaced by

$$
d \mu_{i}=-\bar{S}_{i} d T+\bar{V}_{i} d P+\sum\left(\frac{\partial \mu_{i}}{\partial N_{i}}\right) d N_{i}+\left(\frac{\partial \mu_{i}}{\partial q}\right) d q
$$

where all the partial coefficients except the last are defined at constant $T, P, n_{j}$ and $q$.

The relationship between potential and charge is given as

$$
\Delta \psi=\frac{q}{D}
$$

where $D$ is the dielectric constant. Substituting this equation in equation (1-178), we find that

$$
d G=-S d^{\prime} T^{\prime}+V d P+\sum \mu_{i} d n_{i}+\left(\frac{q}{D}\right) d q
$$

By applying cross-differentiation formulas to equation (1-182), we obtain equations of the type

$$
\begin{array}{ll}
\left(\frac{\partial \mu_{i}}{\partial q}\right)_{n_{i}}=-\frac{1}{D^{2}}\left(\frac{\partial D}{\partial n_{i}}\right)_{q} & \left(T, P, n_{j} \text { constant }\right) \\
\left(\frac{\partial V}{\partial q}\right)_{P}=-\frac{q}{D^{2}}\left(\frac{\partial D}{\partial P}\right)_{q} & \left(T, n_{i} \text { constant }\right)
\end{array}
$$

Equation (1-183) gives the dependence of $\mu_{i}$ on $q$. Equation (1-184), giving the volume change, is called the electrostriction formula.

Frequently it is more desirable to work with $\Delta \psi$ as the independent variable than with $q$. To accomplish this we define a function $\mathfrak{D}$ as

$$
D=E+P V-T S-q(\Delta \psi)
$$

Combining this with equation (1-176) leads to

$$
\begin{aligned}
& d \mathfrak{D}=V d P-S d T+\sum \mu_{i} d n_{i}-q d(\Delta \psi) \\
& =V d P-S d T+\sum \mu_{i} d n_{i}-D(\Delta \psi) d(\Delta \psi)
\end{aligned}
$$

Applying cross-differentiation formulas, we obtain equations of the type

$$
\begin{array}{ll}
\left(\frac{\partial \mu_{i}}{\partial \Delta \psi}\right)_{n_{i}}=-(\Delta \psi)\left(\frac{\partial D}{\partial n_{i}}\right)_{\Delta \psi} & \left(T, P, n_{j} \text { constant }\right) \\
\left(\frac{\partial V}{\partial \Delta \psi}\right)_{P}=-(\Delta \psi)\left(\frac{\partial D}{\partial P}\right)_{\Delta \psi} & \left(T, n_{i} \text { constant }\right)
\end{array}
$$

The treatment of equilibrium conditions when the intensive properties are dependent on the field requires careful consideration. Suppose that the chemical reaction

$$
\sum \nu_{A} A \rightarrow \sum \nu_{B} B
$$


is in equilibrium throughout the system. If material between the plates is isolated, a spontaneous process will occur until conditions in the isolated system become the same as they were outside the plates in the original system. By the definitions previously offered, the material between the plates is in incomplete equilibrium. Thus, we cannot apply $d G=0$, which is the condition for complete equilibrium. However, a small displacement of the reaction between the plates will be reversible since the process occurs in incomplete equilibrium. Thus, reversible work is done on surroundings. This is given as

$$
d G=\Delta \psi d q \quad(T, P \text { constant })
$$

Comparing equations (1-178) and (1-190) we find that, at equilibrium,

and

$$
d G=\sum \mu_{i} d n_{i}+\Delta \psi d q=\Delta \psi d q \quad(T, P \text { constant })
$$

$$
\sum \mu_{i} d n_{i}=0 \quad(T, P \text { constant })
$$

If, now, we consider the transfer of $d n_{i}$ moles from inside to outside the plates, we find (by the method previously used)

$$
\mu_{i}(\text { inside })=\mu_{i} \text { (outside) }
$$

or

$$
d \mu_{i}=0
$$

This is in contrast to the result in a gravitational field.

The effect of the field on composition is now readily found. At constant $T$ and $P$ for a binary mixture, we may write, at equilibrium,

$$
d \mu_{i}=\left(\frac{\partial \mu_{i}}{\partial N_{i}}\right) d N_{i}+\left(\frac{\partial \mu_{i}}{\partial \Delta \psi}\right) d(\Delta \psi)=0
$$

If the solution is ideal, $\partial \mu_{i} / \partial N_{i}=R T / N_{i}$, and using equation (1-188) we obtain the formula

$$
\frac{d N_{i}}{d(\Delta \psi)}=\frac{\Delta \psi\left(\frac{\partial D}{\partial n_{i}}\right)_{\Delta \psi}}{\frac{R T}{N_{i}}}
$$

This integrates into the equation

$$
\ln \frac{N_{i}}{N_{i}^{\circ}}=\frac{(\Delta \psi)^{2}}{R T}\left(\frac{\partial D}{\partial n_{i}}\right)_{\Delta \psi}
$$

where $N^{\circ}{ }_{i}$ is the mole fraction outside the plates.

To complete this treatment of electrostatic field, heat effects will be considered. If a chemical reaction is in incomplete equilibrium between the plates, a small displacement will be reversible, and we know directly from the Second Law that $q$ (heat) $=T d S$. Thus, to find the reversible heat effect, we must find the effect of the field on $S$. At constant $P$ and $n_{i}$, equation (1-187) becomes

$$
d \supseteqq=-S d T-D(\Delta \psi) d(\Delta \psi) \quad\left(P, n_{i} \text { constant }\right)
$$

By direct application of Euler's theorem we obtain the equation:

$$
\left(\frac{\partial S}{\partial \Delta \psi}\right)_{T}=\Delta \psi\left(\frac{\partial D}{\partial T}\right)_{\Delta \psi} \quad\left(P, n_{i} \text { constant }\right)
$$


Thus, by measuring $\partial D / \partial T$, the reversible heat effect of the field can be found. It should be noted that, unlike a gravitational field, the electrostatic field is nonconservative.

The irreversible heat is also affected by the field. Without presenting any details, it can be shown that at constant $P$ and charge, $d H=q$ (heat) for a free-running chemical reaction. Further, by methods similar to those previously used, we find that

$$
\left(\frac{\partial \bar{H}_{i}}{\partial \Delta \psi}\right)_{T, P, n_{i}}=(\Delta \psi) T\left[\frac{\partial}{\partial T}\left(\frac{\partial D}{\partial n_{i}}\right)\right]-\frac{\Delta \psi}{D^{2}}\left(\frac{\partial D}{\partial n_{i}}\right)
$$

Thus, if $\partial D / \partial n_{i}$ and its temperature dependence are known, the effect of the field on the irreversible heat can be found.

\section{Surface area}

In dealing with soil colloids, surface effects are of paramount importance. One might expect to find, then, that the application of equations involving surface area had received widespread attention. This is not the case, apparently owing to the lack of information on surface tension. However, we will develop the formulas here as they do lead to important equations for capillarity.

The treatment is begun by introducing the area of the system into the expression for $d E$ :

$$
d E=T d S-P d V+\sum \mu_{i} d n_{i}+\left(\frac{\partial E}{\partial A}\right)_{S, V, n_{i}} d A
$$

where $A$ is the surface area. We next define the "surface tension," $\sigma$, as

$$
\left(\frac{\partial E}{\partial A}\right)_{S, V, n_{i}}=\sigma
$$

Resorting to the definition of $G$, we find that

$$
d G=-S d T+V d P+\sum \mu_{i} d n_{i}+\sigma d A
$$

Thus,

and

$$
\left(\frac{\partial G}{\partial n_{i}}\right)_{T, P, n_{j}, A}=\bar{G}_{i}=\mu_{i}
$$

$$
\left(\frac{\partial G}{\partial A}\right)_{T, P, n_{i}}=\sigma
$$

From equation (1-203) we obtain, by direct application of Euler's theorem,

and

$$
\left(\frac{\partial \mu_{i}}{\partial A}\right)_{T, P, n_{i}}=\left(\frac{\partial \sigma}{\partial n_{i}}\right)_{T, P, A, n_{j}}
$$

$$
\left(\frac{\partial V}{\partial A}\right)_{P, T, n_{i}}=\left(\frac{\partial \sigma}{\partial P}\right)_{T, A, n_{i}}
$$

Equation (1-206) is called the Gibbs adsorption isotherm. It enables one to find the dependence of $\mu_{i}$ on $A$ from measured values of $\partial \sigma / \partial n_{i}$.

In deriving these formulas, it has been assumed that the system is constrained to some arbitrary shape as the result of interaction with the surroundings. Thus, $A$ is independent of the other extensive variables and the formulas are parallel to those 
for the electrostatic field. This parallelism can be further extended by realizing that, if the constrained surface area changes by $d A$ at constant $T$ and $P$, an amount of work $-\sigma d A$ must be done by the system on the surroundings. This is the reversible work term for incomplete equilibrium. Therefore,

$$
d G=\sigma d A \quad(T, P \text { constant })
$$

is the incomplete equilibrium condition. Comparison with equation (1-203) leads to the additional equilibrium condition

$$
\sum \mu_{i} d n_{i}=0
$$

Assume now, that the system is a liquid constrained to a given shape by a solid but porous mold which is saturated with the liquid, the whole system being in incomplete equilibrium. By considering the transfer of $d n_{i}$ moles between the phases we find by the method previously used that

$$
d \mu_{i}=0
$$

If all constraint is removed from the surface area, we must regain the condition for complete equilibrium expressed in equation (1-23). Thus, we find that

$$
d G=\sum \mu_{i} d n_{i}+\sigma d A=0 \quad(T, P \text { constant })
$$

However, in view of equation (1-209), this leads to the formula

$$
\sigma d A=0
$$

Equation (1-212) is the condition for either a maximum or a minimum in the value of $A$. Thus, on physical grounds we conclude that if all constraint is removed from a surface, spontaneous processes occur until $A$ is a minimum. For a liquid, it can be shown from geometry that this is a sphere.

The constrained equilibrium condition, equation (1-210), may be used to find the effect of area on a binary mixture. For a change of composition and area in incomplete equilibrium we obtain

$$
d \mu_{i}=\left(\frac{\partial \mu_{i}}{\partial N_{i}}\right)_{A} d N_{i}+\left(\frac{\partial \mu_{i}}{\partial A}\right)_{n_{i}} d A=0 \quad(T, P \text { constant })
$$

Using equation (1-206) and assuming an ideal mixture, we find that

$$
\begin{gathered}
\left(\frac{R T}{N_{i}}\right) d N_{i}+\left(\frac{\partial \sigma}{\partial n_{i}}\right) d A=0 \\
R T \frac{d N_{i}}{N_{i}}=-\left(\frac{\partial \sigma}{\partial n_{i}}\right) d A
\end{gathered}
$$

Integrating from the spherical condition (where $N_{i}=N^{\circ}{ }_{i}$ and $A_{i}=A^{\circ}{ }_{i}$ ) to a constrained state, the result is

$$
\ln \frac{N_{i}}{N_{i}^{\circ}}=-\left(A-A^{\circ}\right)\left(\frac{\partial \sigma}{\partial n_{i}}\right)
$$

Usually, $\partial \sigma / \partial n_{i}$ is very small so that $A$ must be large before $N_{i}$ differs from $N^{\circ}{ }_{i}$. This is precisely the case when the constrained state is colloidal so that composition effects are generally noticeable only in colloidal phases. 
The entire treatment of surface area changes when a specifically spherical shape is always imposed on the system. The area is no longer an independent variable but is uniquely related to the volume:

$$
\begin{aligned}
& V=\frac{4}{3} \pi r^{3} \\
& A=4 \pi r^{2} \\
& \frac{d A}{d V}=\frac{2}{r}
\end{aligned}
$$

Thus, the internal energy equation becomes

$$
\begin{aligned}
d E & =T d S-P d V+\sum \mu_{i} d n_{i}+\sigma d A \\
& =T d S-P d V+\sum \mu_{i} d n_{i}+\frac{2 \sigma d V}{r}
\end{aligned}
$$

In deriving the equations for a sphere, special attention must again be given to the pressure term. Our usage throughout has been that $P$ stands for the pressure in the environment. However, in the present case we specifically wish to derive the excess pressure in the sphere. To avoid confusion we will denote the pressure of the surroundings by $P^{\circ}$. Thus,

$$
d E=T d S-P^{\circ} d V+\sum \mu_{i} d n_{i}+\frac{2 \sigma d V}{r}
$$

The definition of $G$ becomes

So that

$$
G=E+P^{\circ} V-T S
$$

$$
d G=-S d T+V d P^{\circ}+\sum \mu_{i} d n_{i}+\frac{2 \sigma d V}{r}
$$

Then,

$$
\left(\frac{\partial G}{\partial n_{i}}\right)_{T, P^{\circ}, n_{i}}=\mu_{i}+\frac{2 \sigma}{r}\left(\frac{\partial V}{\partial n_{i}}\right)_{T, P^{\circ}, n_{j}}
$$

or

$$
\bar{G}_{i}=\mu_{i}+\frac{2 \sigma \bar{V}_{i}}{r}
$$

Thus far, the treatment has involved an additional variable which is not independent, and the formulas are parallel to the gravitational case (for example, $\left.\bar{G}_{i} \neq \mu_{i}\right)$. In the gravitational case, the significance of $\mu_{i}$ is found by realizing that the properties of matter, and hence $\mu_{i}$, are independent of the field. In the present case, a similar, but not identical, situation exists. $\mu_{i}$ is independent of area, and therefore of the equilibrium internal properties of the sphere. Thus, while $\mu_{i}$ is of course dependent on $P^{\circ}$, it is independent of $r$ and of $P$, the equilibrium internal pressure. Thus, for a one component system, $\mu_{i}=\bar{G}^{\circ}{ }_{i}$, where $\bar{G}^{\circ}{ }_{i}$ refers to $r=\infty$. From equation (1-220) we find that

$$
\bar{G}_{i}-\bar{G}_{i}^{\circ}=\frac{2 \sigma \bar{V}_{i}}{r}
$$

The partial molal free energy, however, does depend on the area and also upon the equilibrium internal properties. Thus,

$$
\frac{\partial \bar{G}_{i}}{\partial P}=\bar{V}_{i}
$$


and integration gives

$$
\bar{G}_{i}-\bar{G}_{i}^{\circ}=\bar{V}_{i}\left(P-P^{\circ}\right)
$$

Equating formulas (1-221) and (1-223), we find that

$$
P-P^{\circ}=\frac{2 \sigma}{r}
$$

This formula gives the excess pressure in the sphere. We also immediately derive

$$
R T \ln \frac{p}{p}=\frac{2 \sigma \bar{V}}{r}
$$

for the vapor pressure. Since $p>p^{\circ}$, we conclude that the sphere is unstable in the presence of a large flat surface.

\section{Electrochemical systems and galvanic cells}

The thermodynamics of a galvanic cell is straightforward and requires no special consideration. It is only in the treatment of the extrathermodynamics of electrode processes that formulas involving other variables must enter. We first consider the thermodynamics.

Any chemical reaction which involves the transfer of electrons from one chemical species to another is called an oxidation-reduction reaction. Any device by means of which this electron transfer can be effected through an external circuit is called a galvanic (or electrochemical) cell. In general, we will follow the type of nomenclature previously used for a chemical reaction and represent an oxidation-reduction reaction as

$$
\sum \nu_{I} I \rightarrow \sum \nu_{J} J
$$

In this reaction, certain of the $I$ species (called reducing agents) transfer electrons to other $I$ species (called oxidizing agents). We arrange the galvanic cell in such a way that rather than having a direct electron transfer, the reducing agents give up electrons to a system called a cathode, the oxidizing agents receive electrons from another system called an anode, and the electrons are externally transferred from cathode to anode. If, in the external circuit, we introduce a source of electromotive force which exactly opposes the tendency of the electrons to transfer, we establish an equilibrium condition. However, the equilibrium is incomplete since a spontaneous process would occur if the oxidizing and reducing agents were brought together. If $n$ Faradays of electrons are transferred at constant $T$ and $P$, in a condition only infinitesimally removed from this condition of incomplete equilibrium, the reversible work is given by the formula

$$
\Delta G=-n F_{y} \mathcal{E}
$$

where $F_{y}$ is the Faraday constant and $\varepsilon$ is the equilibrium emf. Following methods exactly parallel to those used in the derivation of equation (1-77), we find that

$$
\Delta G-\Delta G^{\circ}=R T \ln \frac{\Pi\left(a_{J}\right)^{\nu_{J}}}{\Pi\left(a_{I}\right)^{\nu_{I}}}
$$

Substituting equation (1-226), we derive

$$
\varepsilon=\varepsilon^{\circ}-\frac{R T}{n F_{y}} \ln \frac{\mathrm{II}\left(a_{J}\right)^{v_{J}}}{\Pi\left(a_{I}\right)^{\nu_{I}}}
$$


At complete equilibrium for the oxidation-reduction reaction, $\Delta G=\varepsilon=0$ and, from equation (1-228), we find that

$$
\varepsilon^{\circ}=\frac{R T}{n F_{y}} \ln K
$$

where $K$ is the equilibrium constant.

From equation (1-228) we see that the emf of a galvanic cell depends upon the activity of every chemical species in the oxidation-reduction reaction (cell reaction), and the activity of every such species must be known before $\varepsilon$ can be calculated. Alternatively, if $\mathcal{E}$ is measured, and one wishes to calculate the activity of a given species, $a_{i}$, the activity of every other species, $a_{j}$, must be known.

We next turn to the extrathermodynamics of galvanic cells. Imagine that a piece of metallic zinc dips into a solution containing $\mathrm{ZnCl}_{2}$. Within the metal, zinc atoms tend to dissociate according to the reaction

$$
\mathrm{Zn}=\mathrm{Zn}^{++}+2 e^{-}
$$

It is postulated that within the metal, this process is in complete equilibrium, so that

$$
\Delta G=\bar{G}_{\mathrm{Zn}^{++}}+2 \bar{G}_{e^{-}}-\bar{G}_{\mathrm{Zn}}=0
$$

It is further postulated that a condition called "electrochemical equilibrium" exists by assuming that the zinc ions in the metal come to equilibrium with the zinc ions in the solution. Therefore,

$$
\bar{G}_{\mathrm{Zn}^{++}}(\text {metal })=\bar{G}_{\mathrm{Zn}^{++}}(\text {solution })
$$

We now inquire into the mechanism by which electrochemical equilibrium is established. Upon initial contact between the metal and the solution, $\mathrm{Zn}^{++}$ions tend to pass from the metal to the solution. In doing so, electroneutrality is violated since an excess of $\mathrm{Zn}^{++}$ions is now in solution and an excess of electrons is left in the metal. Thus, an electrostatic charge is built up in each phase. This process continues until an electric potential difference is established between the phases which offsets the tendency of $\mathrm{Zn}^{++}$to move from the metal to the solution. This is electrochemical equilibrium.

The potential difference between the phases at equilibrium is called the "Nernst potential." It is also called the "interface potential," because the excess $\mathrm{Zn}{ }^{++}$and electrons form a microscopic double layer at the boundary between the two phases. The number of the excess charges is extremely small, well below the limit of chemical detection. Thus, although one never notices a deviation from electroneutrality by chemical analysis, the presence of interface potentials accounts for galvanic action.

In order to develop equations for the interface potential, we must introduce the electrostatic charge into the thermodynamic formulas. This is done by writing

$$
d E=T d S-P d V+\sum \mu_{i} d n_{i}+\psi d q
$$

where $\psi$ is the electric potential and $q$ is the charge on the phase. In the present case, $q$ is not independent. The charge on a phase which results from electrochemical processes is given as

$$
q=\sum z_{i} F_{y} n_{i}
$$

which is simply the difference between the number of positive and negative charges. 
Therefore,

$$
d q=\sum z_{i} F_{y} d n_{i}
$$

Substituting this result in equation (1-232), we find that

$$
d E=T d S-P d V+\sum\left(\mu_{i}+z_{i} F_{y} \psi\right) d n_{i}
$$

Using the definition of $G$ we find that

and

$$
d G=-S d T+V d P+\sum\left(\mu_{i}+z_{i} F_{y} \psi\right) d n_{i}
$$

$$
\bar{G}_{i}=\mu_{i}+z_{i} F_{y} \psi
$$

Thus, as in the gravitational case, $\mu_{i}$ is not equal to $\bar{G}_{i}$ for charged species. Also, in analogy with the gravitational case, the value of $\mu_{i}$ is not in general affected by $\psi$, since the excess of charge is entirely confined to the surface of the phase. In the example given above, $\mu_{i}$ for $\mathrm{Zn}^{++}$ions in the bulk of the liquid phase is unaffected by the presence or absence of the metal. In dilute solution, $\mu_{i}$ for the ions in solution could be found from DH theory whether the metal is present or not. Thus, $\mu_{i}$ is independent of $\psi$. This is not to say that we will not find a relationship between $\mu_{i}$ and $\psi$ in an electrochemical system at equilibrium. By imposing the equilibrium condition we obtain just such a relationship, in the same way that, although $\mu_{i}$ is independent of $h$ in a gravitational system, we nevertheless find $d \mu_{i} / d h$ at equilibrium.

With this understanding, we may apply equation (1-236) to equation (1-230) to obtain

$$
\left(\mu_{\mathrm{Zn}^{++}}+2 F_{y} \psi_{\mathrm{S}}\right)+2\left(\mu_{e^{-}}-F_{y} \psi_{M}\right)-\mu_{\mathrm{Zn}}=0
$$

where subscript $S$ refers to solution and subscript $M$ refers to metal. Solving for the interface potential, we find that

$$
2 F_{y}\left(\psi_{S}-\psi_{M}\right)=2 F_{y}(\Delta \psi)=\mu_{\mathrm{Zn}_{\mathrm{n}}}-\mu_{\mathrm{Zn}_{\mathrm{n}}{ }^{++}}-2 \mu_{e^{-}}
$$

Equation (1-238) may be written for the case where the zinc, zinc ions, and electrons are in their standard states:

$$
\dot{2 F} F_{y}\left(\Delta \psi^{\circ}\right)=\mu_{\mathrm{Zn}}^{\circ}+\mu_{\mathrm{Zn}^{++}}^{\circ}+2 \mu^{\circ} e^{-}
$$

Now, the zinc metal is of invarient composition so that $\mu^{\circ} \mathrm{Zn}_{\mathrm{n}}=\mu_{\mathrm{Zn}}$ and $\mu_{e^{-}}^{\circ}=\mu_{e^{-}}$. Thus, upon subtracting equation (1-239) from equation (1-238), we obtain

$$
2 F_{y}(\Delta \psi)-2 F_{y}\left(\Delta \psi^{\circ}\right)=-\left(\mu_{\mathrm{Zn}^{++}}-\mu_{\mathrm{Zn}^{++}}^{\circ}\right)
$$

From the definition of ionic activity used in the DH theory (1-107), we find that

$$
\Delta \psi=\Delta \psi^{\circ}-\frac{R T}{2 F_{y}} \ln a_{\mathrm{Zn}^{++}}
$$

This is the well-known expression for the Nernst potential. We may generalize by saying that if an electrode reaction is written

$$
\sum \nu_{A} A \rightarrow \sum \nu_{B} B+n e^{-}
$$

the corresponding Nernst potential is

$$
\Delta \psi=\Delta \psi^{\circ}-\frac{R T}{n F_{y}} \ln \frac{\Pi\left(a_{B}\right)^{v_{B}}}{\Pi\left(a_{A}\right)^{\nu_{A}}}
$$


We next suppose that two different electrodes are immersed in the same solution and that each electrode has identical metallic terminals. In general, Nernst potentials will be established at each electrode in such a way that the partial molal free energy of the electrons in each metal differs. Consequently, if the electrodes are connected, current flows. On open circuit, the difference in $\bar{G}_{i}$ for the electrons between the metals may be found as follows:

$$
\begin{aligned}
\bar{G}_{e^{-I}} & =\mu_{e}^{-\mathrm{I}}-F_{y} \psi^{\mathrm{I}} \\
\bar{G}_{e}^{-\mathrm{II}} & =\mu_{e}^{-\mathrm{II}}-F_{y} \psi^{\mathrm{II}}
\end{aligned}
$$

where superscripts I and II denote the electrodes. Since the metals are identical, $\mu_{e^{-I}}=\mu_{e^{-I I}}$, and

$$
\bar{G}_{e}^{-\mathrm{I}}-\bar{G}_{e^{-\mathrm{II}}}=-F_{y}\left(\psi^{\mathrm{I}}-\psi^{\mathrm{II}}\right)
$$

Thus, a measurement of $\bar{G}_{e}^{-\mathrm{I}}-\bar{G}_{e^{-\mathrm{II}}}$ gives $\psi^{\mathrm{I}}-\psi^{\mathrm{II}}$. As strongly emphasized by Guggenheim (1949), equation (1-245) applies only to identical metals. One cannot measure the electrical potential difference between two phases which differ in chemical composition.

At each of the electrodes, we have potential differences defined by $\Delta \psi^{\mathrm{I}}=\psi^{S}-\psi^{\mathrm{I}}$ and $\Delta \psi^{\mathrm{II}}=\psi^{S}-\psi^{\mathrm{II}}$. Therefore,

$$
\Delta \psi^{\mathrm{II}}-\Delta \psi^{\mathrm{I}}={ }^{\prime}\left(\psi^{\mathrm{I}}-\psi^{\mathrm{II}}\right)=\varepsilon
$$

which is the measured cell emf. The difference between the two electrode reactions, written so as to involve the same number of electrons, is the net cell reaction. This may again be written

$$
\sum \nu_{I} I \rightarrow \sum \nu_{J} J
$$

Therefore, if equation (1-242) is applied to each electrode and substituted into equation (1-246), we obtain

$$
\varepsilon=\left(\Delta \psi^{\mathrm{II}}\right)^{\circ}-\left(\Delta \psi^{\mathrm{I}}\right)^{\circ}-\frac{R T}{n F_{y}} \ln \frac{\Pi\left(a_{J}\right)^{\nu_{J}}}{\Pi\left(a_{I}\right)^{\nu_{I}}}
$$

where $\mathcal{E}$ is the measured cell emf. Putting $\left(\Delta \psi^{\mathrm{II}}\right)^{\circ}-\left(\Delta \psi^{\mathrm{I}}\right)^{\circ}=\mathcal{E}^{\circ}$, we regain equation (1-228). The method of treatment is thus consistent with thermodynamics.

Thus far we have been largely concerned with potential differences between metals and solutions. In fact, electric potentials may arise between any two phases of different chemical composition which contain charged species. We now turn attention to the boundary between two liquid phases.

Consider the following cell:

$$
\left.E\right|^{A} m_{i} m_{m_{i}+d m_{i}}^{J} E
$$

$E$ represents identical electrodes dipping into two solutions, I and II, which are mixtures of ions differing only infinitesimally in composition. Each contains an ion, say $\mathrm{M}^{+}$, to which the $E$ are reversible.

If the electrodes were connected, current would flow through the system. We will suppose that positive ions flow from left to right and negative ions from right to left. We now define the ionic "transference number," $t_{i}$, as the fraction of the 
current carried across $J$ by each ionic species. We also define the ionic "mobility," $v_{i}$, as the velocity of an ion in centimeters per second when a field strength of one volt per centimeter is applied. By a straightforward derivation which is omitted here (see MacInnis, 1939) we find

$$
t_{i}=\frac{\left|z_{i}\right| m_{i} v_{i}}{\sum\left|z_{i}\right| m_{i} v_{i}}
$$

When one Faraday of current flows across the boundary $J$ we will thus find that $t_{i} / z_{i}$ moles of each ion move from left to right $\left(z_{i}<0\right.$ for anions $)$. Thus, the transference process can be written

$$
\sum^{\mathrm{I}} \frac{t_{i}}{z_{i}}\left(I_{i}\right) \rightarrow \sum^{\mathrm{II}} \frac{t_{i}}{z_{i}}\left(I_{i}\right)
$$

where $I_{i}$ denotes an ion species. The free energy change is

$$
\Delta G=\sum \frac{t_{i}}{z_{i}} \bar{G}_{i}^{\mathrm{II}}-\sum \frac{t_{i}}{z_{i}} \bar{G}_{i}^{\mathrm{I}}
$$

However, we have supposed that the concentrations differ only infinitesimally so that the $\bar{G}_{i}$ differ only infinitesimally, and

$$
d G=\sum \frac{t_{i}}{z_{i}} d \bar{G}_{i}
$$

Now, when phases I and II are initially brought into contact with the circuit open, we postulate that small currents flow according to the above transference process until electrochemical equilibrium is established. (This will not establish diffusion equilibrium, and equation (1-249) is not the $\Delta G$ for diffusion.) This requires that

$$
d G=\sum \frac{t_{i}}{z_{i}} d \bar{G}_{i}=0
$$

Separating $\bar{G}_{i}$ into chemical and electrical potential terms, we obtain

$$
\begin{aligned}
& \sum \frac{t_{i}}{z_{i}}\left(d \mu_{i}+z_{i} F_{y} d \psi\right)=0 \\
& -\sum t_{i} F_{y} d \psi=\sum \frac{t_{i}}{z_{i}} d \mu_{i}
\end{aligned}
$$

Using the definition of ionic activities, we obtain the equation

$$
-F_{y} d \psi \sum t_{i}=\sum \frac{t_{i}}{z_{i}} R T d \ln a_{i}
$$

Furthermore, $\sum t_{i}=1$, since the transference numbers are defined as fractions. Thus, we find

$$
d \psi=-\sum \frac{t_{i}}{z_{i}} \frac{R T}{F_{y}} d \ln a_{i}
$$

In the event that I and II differ by finite concentrations, we integrate and find that

$$
\Delta \psi_{\mathrm{J}}=-\int_{\mathrm{I}}^{\mathrm{II}} \sum \frac{t_{i}}{z_{i}} \frac{R T}{F_{y}} d \ln a_{i}
$$


Subscript $J$ denotes the electric potential difference at the liquid-liquid boundary. The $t_{i}$ are clearly dependent on the concentrations, but on substituting equation (1-248), we obtain

$$
\Delta \psi_{\mathrm{J}}=-\frac{R T}{F_{y}} \int_{\mathrm{I}}^{\mathrm{II}} \sum \frac{\left|z_{i}\right| m_{i} v_{i}}{z_{i} \sum\left|z_{i}\right| m_{i} v_{i}} d \ln a_{i}
$$

The solution of equation (1-257) requires knowledge of ionic activity coefficients and of the dependence of the ionic mobilities on composition, neither of which is subject to direct experimental measurement. In very dilute solution, one may assume $\gamma_{i}=1$ and $v_{i}=$ constant. The result is the well-known Henderson equation:

$$
\Delta \psi_{J}=\frac{R T}{F_{y}} \frac{\sum \frac{v_{i}}{z_{i}}\left(N_{i}{ }^{\mathrm{II}}-N_{i}^{\mathrm{I}}\right)}{\sum v_{i}\left(N_{i} \mathrm{II}-N_{i} \mathrm{I}\right)} \ln \frac{\sum v_{i} N_{i}}{\sum v_{i} N_{i} \mathrm{I}}
$$

where the $N_{i}$ are normalities. Obviously, it is of limited application.

Equation (1-256) is also of interest for the case in which phases I and II contain only a single uni-univalent electrolyte. For example, suppose we have the cell

$$
\begin{array}{ll|lll} 
& & & \text { J } & \\
H_{2} & & \mathrm{HCl} & \mathrm{HCl} & \\
& & & & \mathrm{H}_{2}
\end{array}
$$

By previous methods we find that

and

$$
\Delta \psi_{A}=\Delta \psi^{\circ}-\frac{R T}{F_{y}} \ln a_{\mathrm{H}^{+}}{ }^{\mathrm{I}}
$$

Thus,

$$
\Delta \psi_{B}=\Delta \psi^{\circ}-\frac{R T}{F_{y}} \ln a_{\mathrm{H}^{+1}}
$$

$$
\Delta \psi_{A}-\Delta \psi_{B}=-\frac{R T}{F_{y}} \ln \frac{a_{\mathrm{H}^{+}}}{a_{\mathrm{H}^{+}}}
$$

Applying equation (1-257) to $J$, we obtain the formula

$$
\Delta \psi_{J}=-\int_{\mathrm{I}}^{\mathrm{II}} t_{\mathrm{H}^{+}} \frac{R T}{F_{y}} d \ln a_{\mathrm{H}^{+}}+\int_{\mathrm{I}}^{\mathrm{II}} t_{\mathrm{Cl}^{-}} \frac{R T}{F_{y}} d \ln a_{\mathrm{Cl}^{-}}
$$

In the present case we assume that the $t_{i}$ are independent of composition (since $\left.t_{i}=v_{i} / \sum v_{i}\right)$, and

$$
\Delta \psi_{J}=-t_{\mathrm{H}^{+}} \frac{R T}{F_{y}} \ln \frac{a_{\mathrm{H}^{+}} \mathrm{II}}{a_{\mathrm{H}^{+}}}+t_{\mathrm{Cl}^{-}} \frac{R T}{F_{y}} \ln \frac{a_{\mathrm{Cl}^{-1 \mathrm{I}}}}{a_{\mathrm{Cl}^{-\mathrm{I}}}}
$$

Recalling that $t_{\mathrm{H}^{+}}=1-t_{\mathrm{Cl}^{-}}$and $a_{\mathrm{HCl}}=a_{\mathrm{H}^{+}} \cdot a_{\mathrm{Cl}^{-}}$, equation (1-263) becomes

Since

$$
\Delta \psi_{J}=-\frac{R T}{F_{y}} \ln \frac{a_{\mathrm{H}^{+} \mathrm{II}}}{a_{\mathrm{H}^{+}}}+t_{\mathrm{Cl}^{-}} \frac{R T}{F_{y}} \ln \frac{a_{\mathrm{HCl}}{ }^{\mathrm{II}}}{a_{\mathrm{HCl}} \mathrm{I}^{\mathrm{I}}}
$$

$$
\begin{aligned}
\Delta \psi_{A} & =\psi^{\mathrm{I}}-\psi_{A} \\
\Delta \psi_{B} & =\psi^{\mathrm{II}}-\psi_{B} \\
\Delta \psi_{J} & =\psi^{\mathrm{II}}-\psi^{\mathrm{I}}
\end{aligned}
$$


it follows that

$$
\varepsilon=\psi_{B}-\psi_{A}=\Delta \psi_{A}-\Delta \psi_{B}-\Delta \psi_{J}
$$

where $\mathcal{E}$ is the measured cell emf. Using equations (1-261) and (1-264), we find that

$$
\varepsilon=-t_{\mathrm{Cl}}-\frac{R T}{F_{y}} \ln \frac{a_{\mathrm{HCl}}{ }^{\mathrm{II}}}{a_{\mathrm{HCl}{ }^{\mathrm{I}}}}
$$

Thus, in the case where only one electrolyte is present at different concentrations ("concentration cell"), the cell emf can be found on the single assumption that the $v_{i}$, and hence $t_{i}$, is independent of composition. In writing equation (1-263) it is often further assumed that $a_{\mathrm{H}^{+}}=a_{\mathrm{Cl}^{-}}=a_{ \pm \mathrm{HCl}}$, so that equation (1-263) for $\Delta \psi_{J}$ becomes

$$
\Delta \psi_{J}=\left(1-2 t_{\mathrm{H}^{+}}\right) \frac{R T}{F_{y}} \ln \frac{a_{\mathrm{HCl}}{ }^{\mathrm{II}}}{a_{\mathrm{HCl}} \mathrm{I}^{\mathrm{I}}}
$$

One of the most widely used galvanic cells is the $\mathrm{pH}$ meter. The electrode assembly of this apparatus may be represented as follows:

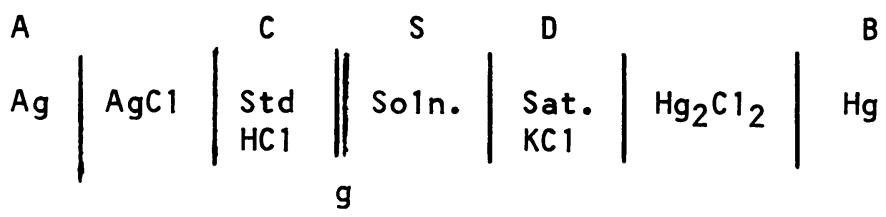

In this assembly, the $\mathrm{Ag}-\mathrm{AgCl}$ electrode is immersed in a standard $\mathrm{HCl}$ solution of fixed composition. Contact with the solution $S$ is made with a glass membrane (g) which is permeable to $\mathrm{H}^{+}$only. The calomel electrode on the left is immersed in a solution of saturated $\mathrm{KCl}$ which also makes the other contact with the solution.

In general, the composition of the solution in $S$ is not known so that formal thermodynamics cannot be applied. However, the more powerful extrathermodynamic method is available.

At the $\mathrm{Ag}-\mathrm{AgCl}$ electrode the reaction is

$$
\mathrm{Ag}+\mathrm{Cl}^{-}=\mathrm{AgCl}+e^{-}
$$

The corresponding electrode potential is

$$
\Delta \psi_{A}=\psi^{C}-\psi^{A}=\Delta \psi_{A}^{\circ}+\frac{R T}{F_{y}} \ln a_{\mathrm{Cl}^{-}}{ }^{C}
$$

At the calomel electrode the reaction is

$$
2 \mathrm{Hg}+2 \mathrm{Cl}^{-}=\mathrm{Hg}_{2} \mathrm{Cl}_{2}+2 e^{-}
$$

The corresponding electrode potential is

$$
\Delta \psi_{B}=\psi^{D}-\psi^{B}=\Delta \psi^{\circ}{ }_{B}+\frac{R T}{F_{y}} \ln a_{\mathrm{Cl}^{-}}{ }^{D}
$$

Assuming electrochemical equilibrium for $\mathrm{H}^{+}$across the glass membrane, we find that

$$
\Delta \psi_{g}=\psi^{S}-\psi^{c}=\frac{R T}{F_{y}} \ln \frac{a_{\mathrm{H}^{+}}{ }^{c}}{a_{\mathrm{H}^{+}}{ }^{S}}
$$

At the boundary between the solution and the saturated $\mathrm{KCl}$ bridge we write

$$
\Delta \psi_{J}=\psi^{D}-\psi^{S}
$$


The net cell emf is

$$
\begin{aligned}
\mathcal{E} & =\psi^{B}-\psi^{A} \\
& =\Delta \psi_{A}-\Delta \psi_{B}+\left(\psi^{D}-\psi^{\prime}\right) \\
& =\Delta \psi_{A}-\Delta \psi_{B}+\left(\psi^{D}-\psi^{S}\right)+\left(\psi^{S}-\psi^{C}\right) \\
& =\Delta \psi_{A}-\Delta \psi_{B}+\Delta \psi_{J}+\Delta \psi_{g}
\end{aligned}
$$

Substituting equations (1-268), (1-269) and (1-270) in equation (1-273), we obtain the formula

$$
\mathcal{E}=\Delta \psi^{\circ}{ }_{A}-\Delta \psi^{\circ}{ }_{B}+\frac{R T}{F_{y}} \ln \frac{a_{\mathrm{Cl}^{-}}{ }^{c}}{a_{\mathrm{Cl}^{-}}{ }^{D}}+\frac{R T}{F_{y}} \ln \frac{a_{\mathrm{H}^{+}}{ }}{a_{\mathrm{H}^{+}}{ }^{2}}+\Delta \psi^{\prime}
$$

Since $a_{\mathrm{Cl}^{-D}}, a_{\mathrm{C1}^{-}}{ }^{C}$ and $a_{\mathrm{H}^{+}}{ }^{C}$ are all fixed, we may define the standard emf as

$$
\mathcal{E}^{\circ}(\mathrm{pH})=\Delta \psi_{A}^{\circ}-\Delta \psi^{\circ}{ }_{B}+\frac{R T}{F_{y}} \ln \frac{a_{\mathrm{H}^{+}}{ }^{C} \cdot a_{\mathrm{Cl}^{-}}{ }^{C}}{a_{\mathrm{C} 1^{-}}{ }^{-}}
$$

Equation (1-274) then becomes

The $\mathrm{pH}$ is defined as

$$
\mathcal{E}=\varepsilon^{\circ}(\mathrm{pH})-\frac{R T}{F_{y}} \ln a_{\mathrm{H}^{+}}{ }^{s}+\Delta \psi_{J}
$$

$$
\mathrm{pH}=-\log _{10} a_{\mathrm{H}^{+}}
$$

so that equation (1-276) becomes

Solving for the $\mathrm{pH}$, we obtain

$$
\varepsilon=\varepsilon^{\circ}(\mathrm{pH})+\frac{R T}{2.3 F_{y}}(\mathrm{pH})+\Delta \psi_{J}
$$

$$
\mathrm{pH}=\frac{\mathcal{E}-\mathcal{E}^{\circ}(\mathrm{pH})-\Delta \psi_{J}}{\frac{R T}{2.3 F}}
$$

When the solution concentration is much lower than that of the bridge, an application of the Henderson equation to the boundary between $S$ and $D$ leads to $\Delta \psi_{J} \cong 0$ since $v_{\mathrm{K}^{+}} \cong v_{\mathrm{Cl}^{-}}$in $D$. Thus, $\varepsilon^{\circ}(\mathrm{pH})$ may be evaluated in principle by extrapolating values of $\mathcal{E}$ to infinite dilution. In the concentration range when $\Delta \psi_{J}$ remains negligible, equation (1-279) becomes

$$
\mathrm{pH}=\frac{\mathcal{E}-\mathcal{E}^{\circ}(\mathrm{pH})}{\frac{R T}{2.3 F_{y}}}
$$

In the range of the $\mathrm{DH}$ theory, values of the $\mathrm{pH}$ found from equation (1-280) are in agreement with those calculated from the theory. Outside this range, we have no means of investigating the accuracy of equation (1-280). This has led some writers to define the $\mathrm{pH}$ according to equation (1-280).

A final point concerning electrochemical systems must be made. We have written in general that

$$
d G=-S d T+V d P+\sum\left(\mu_{i}+z_{i} F_{y} \psi\right) d n_{i}
$$

The fact that the $\mu_{i}$ are independent of $\psi$ has been emphasized. Therefore, if we are dealing with a single phase containing electrolytes, in which any electric potential which characterizes the phase as a whole is incidental, we may abritrarily put $\psi=0$ just as we put $\phi=0$ in the gravitational case. At constant $T$ and $P$, equation (1-281) becomes

$$
d G=\sum \mu_{i} d n_{i}
$$

where the summation is over every ionic species present. 


\section{Section II}

\section{DONNAN THEORY}

\section{Introduction}

The theory of membrane systems developed by Donnan (1911) and generalized by Donnan and Guggenheim (1932), has been widely applied to colloidal systems of both organic and inorganic origin. The concern of the original theory was to develop equations for the distribution of ionic species between two macroscopic phases which are separated by a semipermeable membrane and also to develop equations for the potential difference which may exist at such a membrane. Unfortunately, the expressions "Donnan system" and "Donnan theory" have been used in different ways by different writers.

For certain writers (Davis, 1942; Overbeek, 1956; Babcock, 1960), any macroscopic system of two phases, one containing electrostatically charged colloidal particles and the other a solution, separated by a membrane impermeable to colloid but permeable to ions, may be called a Donnan system. Other writers (Bolt and Peech, 1953) hold a similar view, but contend that there must be volume homogeneity of charge in the suspension before the system can be called a Donnan system or the Donnan theory applied. In both of these approaches, a Donnan system is macroscopic and contains a phase boundary at which macroscopic discontinuities in ionic concentrations occur.

Still other writers (Wiklander, 1955) prefer to speak of the "Donnan distribution" of the ionic concentrations in the immediate vicinity of charged colloidal particles. This distribution does not necessarily involve a discontinuity in the concentrations. One then speaks of the "micro-Donnan system."

In the present chapter, the most general macroscopic nomenclature, as described above, will be adopted. Consider a two-phase system, one phase of which contains an aqueous suspension of charged colloidal particles and an electrolyte (I) and the other an electrolyte only (II):

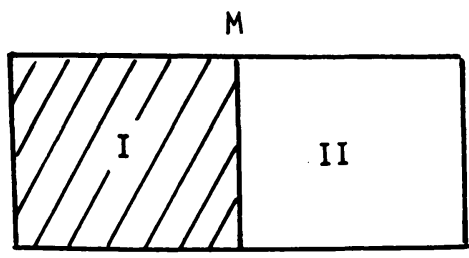

The phases are separated by a membrane, $M$, permeable to electrolytes but not to colloids. We will consider any such system to be a Donnan system. Furthermore, any formulas which apply to this macroscopic system will be called Donnan equations. Thus, we might construct a model in which the microscopic state of the suspension is governed by the double-layer theory and use the Donnan equations to find the macroscopic state, since the theories are not incompatible. Such an effort will be made in Section III. Overbeek (1956) used a similar approach. 


\section{Macroscopic equations and negative adsorption}

Certain equations can be developed with only a minimal number of assumptions. Consider a diffusible electrolyte species $X$ in the system. We may with confidence say that, at equilibrium,

$$
\bar{G}_{X} \mathrm{I}=\bar{G}_{X}{ }^{I I}
$$

Further, if the same standard state is chosen for the electrolyte in the suspension as for electrolyte in the solution, then we also find

$$
a_{X}{ }^{\mathrm{I}}=a_{X} \mathrm{II}
$$

In order to proceed further, it is necessary to go beyond purely macroscopic considerations. It will be recalled that in choosing the standard state for strong electrolytes, it was postulated that Henry's Law applies to ions at infinite dilution. This led to $\gamma_{ \pm}=1$ at infinite dilution. In defining the activity coefficient of electrolyte in a suspension, it is postulated that the electrolyte and the colloid are completely dissociated, so that the mean ionic concentrations are determinable unambiguously. Thus, we may write for the electrolyte

$$
a_{X}{ }^{\mathrm{I}}=\left(\gamma_{ \pm}^{\mathrm{I}} \cdot m_{ \pm}^{\mathrm{I}}\right)^{\nu}
$$

where $\gamma_{ \pm}$and $m_{ \pm}$are given by equations (1-86) and (1-87), respectively. The only limitation on this equation is that the mean ionic concentrations must be determinable. In the event that ions in the suspension obey Henry's Law, $\gamma_{ \pm}{ }^{\mathrm{I}}$ will equal unity at infinite dilution. This will certainly occur if there is volume homogeneity of charge. If not, it must remain for experiment to determine ${\gamma_{ \pm}}^{\mathrm{I}}$ at infinite dilution.

It must be noted that certain potentiometric measurements made in clay suspensions have been interpreted to mean that clays are not completely dissociated (Mattson, 1926; Marshall and Krinbill, 1941). If this is so, equation (2-3) cannot be applied in a straightforward manner. However, in the writer's opinion, the weight of experimental evidence strongly favors the conclusion that for the alkali metals, such as $\mathrm{Na}^{+}$or $\mathrm{K}^{+}$, and the alkaline earths, such as $\mathrm{Mg}^{++}$and $\mathrm{Ca}^{++}$, the ions are completely dissociated from the surface. The alternative is that they are covalently bonded, or in some sense "complexed," by the surface. In general, the data on the rapidity of ion-exchange, the apparent mobilities of ions in suspensions, and the activity coefficients of electrolytes in clay systems, all indicate complete dissociation. We will thus assume that equation (2-3) is unambiguous.

Assume that the suspension contains a single electrolyte which dissociates into $\nu_{+}$cations $\left(X_{+}\right)$and $\nu_{-}$anions $\left(X_{-}\right)$. Using equation (1-90) for the electrolyte in the suspension we derive from equation (2-2)

$$
\left[\gamma_{ \pm}{ }^{\nu} m_{+}{ }^{\nu_{+}} \cdot m_{-}{ }^{\nu-}\right]^{\mathrm{I}}=\left[\gamma_{ \pm}{ }^{\nu} \cdot m_{X^{\nu}}\right]^{\mathrm{II}}
$$

where $m_{i}$ is the mean or bulk ionic concentration. Solving for $\gamma_{ \pm}{ }^{\mathrm{I}}$, we derive

$$
{\gamma_{ \pm}}^{\mathrm{I}}=\frac{{\gamma_{ \pm}}^{\mathrm{II}} m_{\mathrm{X}}{ }^{\mathrm{II}}}{\left[\left(m_{+}{ }^{{ }^{+}} \cdot m_{-}{ }^{\nu_{-}}\right)^{1 / \nu}\right]^{\mathrm{I}}}
$$

Thus, if the quantities on the right-hand side of equation (2-5) are known or meassured, $\gamma_{ \pm}{ }^{\mathrm{I}}$ can be found. This equation is also applicable whenever the ionic molalities in the suspension are determinable. We may also write

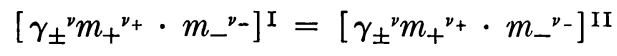


or

$$
\left(\frac{m_{+}{ }^{I}}{m_{+}{ }^{I I}}\right)^{\nu_{+}}=\left(\frac{\gamma_{ \pm}^{\mathrm{II}}}{\gamma_{ \pm}^{\mathrm{I}}}\right)^{\nu}\left(\frac{m_{-}{ }^{\mathrm{II}}}{m_{-}^{\mathrm{I}}}\right)^{\nu_{-}}
$$

Thus, in general the ionic concentrations are not the same in the two phases.

For the most part, we will be interested in negatively charged colloidal systems. In what follows, then, the equations for anion distributions will be found.

It is convenient to rewrite equation $(2-7)$ in the following way. Let $m_{p}$ be the concentration of the particles expressed in equivalents per liter, and $\theta$ be defined as

Then,

$$
\theta=\frac{\gamma_{ \pm}{ }^{\mathrm{II}}}{\gamma_{ \pm}^{\mathrm{I}}}
$$

$$
m_{+}{ }^{\mathrm{I}}=\frac{m_{p}}{z_{+}}+\frac{\left|z_{-}\right| m_{-} \mathrm{I}}{z_{+}}
$$

and equation (2-7) may be written

$$
\theta^{\nu}\left(\frac{m_{p}}{z_{+}}+\frac{\left|z_{-}\right| m_{-}{ }^{\mathrm{I}}}{z_{+}}\right)=\left(m_{+}{ }^{\mathrm{II}}\right)^{\nu+}\left(\frac{m_{-}{ }^{\mathrm{II}}}{m_{-}{ }^{\mathrm{I}}}\right)^{\nu-}
$$

For a symmetrical electrolyte, $\nu_{-}=\nu_{+}=1, \nu=2$, and

$$
\theta^{2}\left(\frac{m_{p}}{z_{+}}+\frac{\left|z_{-}\right| m_{-}{ }^{\mathrm{I}}}{z_{+}}\right)=m_{+} \mathrm{II}\left(\frac{m_{-}{ }^{\mathrm{II}}}{m_{-} \mathrm{I}}\right)
$$

This equation can be solved for the "anion ratio" $(\alpha)$ using $m_{+}{ }^{\text {II }}=m_{-}{ }^{\text {II }}=m_{0}$ :

$$
\alpha=\frac{m_{-}{ }^{\mathrm{II}}}{m_{-}^{\mathrm{I}}}=\frac{2\left|z_{-}\right| \theta m_{0}}{\sqrt{\theta^{2} m^{2}{ }_{p}+4 z_{+}\left|z_{-}\right| m^{2}{ }_{0}}-\theta m_{p}}
$$

We also define the "negative adsorption" of anions as

$$
\beta=\frac{\left|z_{-}\right|\left(m_{-}{ }^{\mathrm{II}}-m_{-}\right)}{m_{p}}
$$

Solving equation (2-11) for $\beta$, we obtain

$$
\beta=\frac{2\left|z_{-}\right| \theta m_{0}+\theta m_{p}-\sqrt{\theta^{2} m_{p}^{2}+4 z_{+}\left|z_{-}\right| m_{0}^{2}}}{2 \theta m_{p}}
$$

It is convenient to introduce the parameter

$$
y=\frac{\left|z_{-}\right| m_{-}{ }^{\text {II }}}{m_{p}}
$$

as an index of the relative amounts of salt and colloid in the system. Equations (2-12) and (2-14) then become

$$
\begin{gathered}
\alpha=\frac{2 y \theta}{\sqrt{\theta^{2}+4 y}-\theta} \\
=\frac{\theta(2 y+1)-\sqrt{\theta^{2}+4 y^{2}}}{2 \theta}
\end{gathered}
$$

Thus, the ratio and the negative adsorption are determined by $y$ and $\theta$. 
In general, $\theta$ will be a marked function of $y$. However, insight into the behavior of Donnan systems under nonideal conditions is gained by plotting $\alpha$ and $\beta$ as functions of $y$ at constant $\theta$. The plots must be considered to represent a series of different exchanger systems in which, as $y$ increases, the exchanger interactions change in such a way that $\theta$ remains constant. The plots are given in figures 1 and 2 . The results for nonideal systems are interesting in that at low values of $\theta, \alpha$ becomes less than unity while $\beta$ goes negative. This means simply that for low values of $\gamma_{ \pm}{ }^{\mathrm{I}}$, due to strong interaction between cations and the colloid, the anion concentration in the suspension must exceed that in the solution in order that the activity of the salt be the same in two phases. That is, owing to strong interaction between cation and colloid, one may get positive anion adsorption in a system of negatively charged particles.

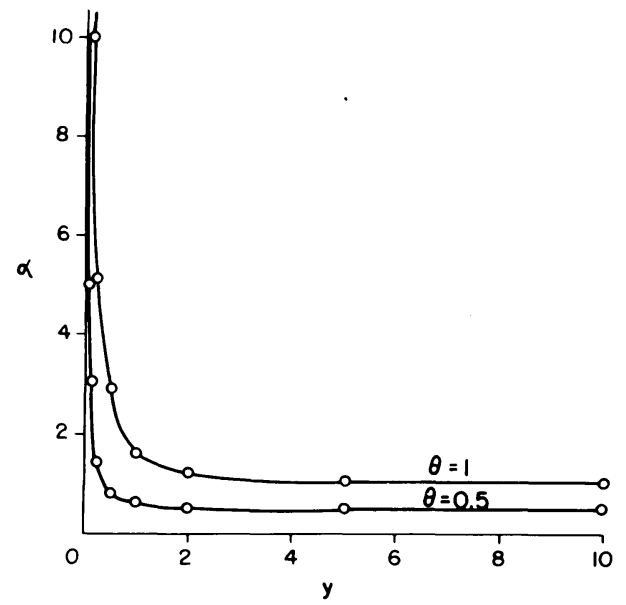

Figure 1. Effect of the relative salt content of a suspension $(y)$ and the anion ratio $(\alpha)$.

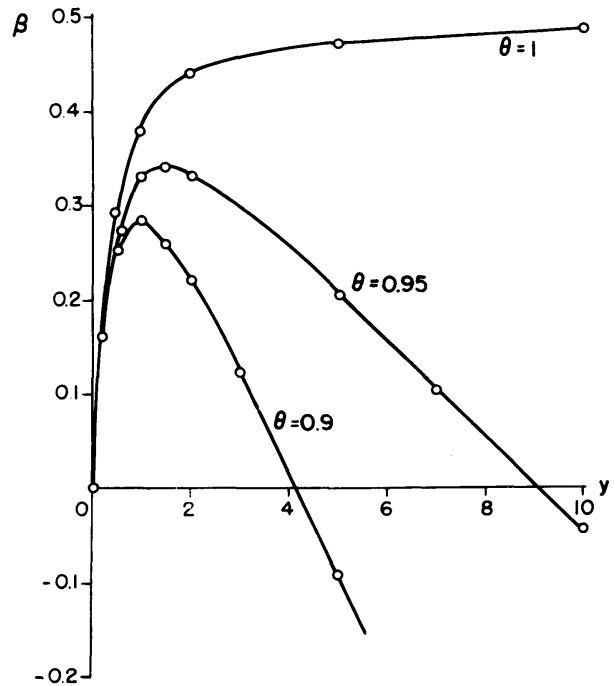

Figure 2. Effect of the relative salt content of a suspension $(y)$ on the negative adsorption $(\beta)$.

\section{Electrochemistry of a Donnan system}

Whenever two phases of different chemical composition, each containing ionic species, are brought into contact, the possibility of an electrostatic interface potential exists. Interface potentials of this type are discussed in Section I under electrochemical systems. In the Donnan system, we have the conditions required for the existence of such an interface potential.

We must consider the possibility, then, that when contact is established between two phases of a Donnan system an electric potential difference may arise at the membrane. Like all potentials in electrochemical systems, this potential would be due to very small excesses of charge in each phase which form a localized double layer at the interface.

For each diffusible ionic species at electrochemical equilibrium

$$
\bar{G}_{i}^{\mathrm{I}}=\bar{G}_{i}^{\mathrm{II}}
$$

Separating $\bar{G}_{i}$ as in equation (1-236) we derive

$$
\mu_{i}^{\mathrm{I}}+z_{i} F_{y} \psi^{\mathrm{I}}=\mu_{i}^{\mathrm{II}}+z_{i} F_{y} \psi^{\mathrm{II}}
$$


The "membrane potential" may now be defined as

$$
\Delta \psi_{m}=\psi^{\mathrm{II}}-\psi^{\mathrm{I}}
$$

Combining equations (2-19) and (2-20), we obtain

$$
\Delta \psi_{m}=\frac{1}{z_{i} F_{y}}\left(\mu_{i}^{\mathrm{I}}-\mu_{i}^{\mathrm{II}}\right)
$$

Using the definition of ionic activity from equation (1-107), we obtain

$$
\Delta \psi_{m}=\frac{R T}{z_{i} F_{y}} \ln \frac{a_{i}^{\mathrm{I}}}{a_{i}^{\mathrm{II}}}
$$

Thus, in general the ionic activities are not the same in the two phases.

In deriving equation (2-22), the method employed is precisely the same as that used to derive interface potentials in a galvanic cell in Section I. Thus, the chemical potentials in equation (2-21) are independent of $\Delta \psi_{m}$. For example, if phase II is dilute, $\mu_{i}{ }^{\mathrm{II}}-\mu^{\circ}{ }_{i}$ could be calculated from the $\mathrm{DH}$ theory whether phase $\mathrm{I}$ is present or not. In both phases all electrostatic interactions within the phase are included in $\mu_{i}$ and in $a_{i}$. Thus, the electrostatic interactions between the ions and the colloid in phase $I$ are included in $\mu_{i}{ }^{3}$

These points require emphasis because the symbols $\mu_{i}$ and $a_{i}$ are sometimes given a different significance in the literature. In writing

$$
\bar{G}_{i}=\mu_{i}+z_{i} F_{y} \psi
$$

for an ion in the suspension, many authors (Low, 1951; Whitney and Peech, 1952) intend $\psi$ to stand for the electric potential in the diffuse layer of ions around the colloidal particle. Since this potential is a function of position and since $\bar{G}_{i}$ is a constant throughout the suspension, $\mu_{i}$ becomes a function of position. Thus, if we write

$$
\mu_{i}-\mu_{i}^{\circ}=R T \ln a_{i}
$$

the activity also becomes a function of position. In this usage, the interaction between the colloid and the ions is not included in $\mu_{i}$.

As discussed in Section I under "Systems involving other variables," the use of $\mu_{i}$ is not arbitrary once one has written equation (1-125). Thus, using $\mu_{i}$ to stand for a quantity which includes only a part of the interactions in phase $I$ is in violation of the basic definition of $\mu_{i}$ in thermodynamics. In respect to the activity, one is free to define this arbitrary function in any manner which is the most convenient, but to define $a_{i}$ such that it is a function of position violates usage in electrochemistry in general and the $\mathrm{DH}$ theory in particular. For example, one does not obtain the conventional formula $(2-22)$ for the membrane potential.

\footnotetext{
${ }^{8}$ Given the existence of equation (2-21), it may seem strange to state that the chemical potentials are independent of $\Delta \psi_{m}$. To make this completely clear, recall the gravitational case. It was carefully stated that $\mu_{i}$ is independent of the gravitational field, since the properties of matter are not affected by the field. However, in a series of phases at equilibrium, we know from equation (1-167) that $d \mu_{i}=M d \phi$, which gives $\mu_{i}$ as a function of $\phi$. This is in no way different from asserting that $T$ and $P$ are independent variables, but then finding the equilibrium pressure of a phase as a function of temperature. Thus, in electrochemical systems, it is precisely by realizing that $\mu_{i}$ is independent of $\Delta \psi_{m}$ that we arrive at the equilibrium condition, equation (2-21).
} 
Therefore, in the nomenclature to be used here:

a) $\Delta \psi_{m}$ is a nonspecific membrane potential resulting from a very small charge separation localized at the membrane.

b) $\mu_{i}$ is independent from $\psi$ and is uniform throughout both solution and suspension. It includes interactions between the ions and the colloid.

c) $a_{i}$ is also independent of $\psi$ and is uniform throughout both solution and suspension. It also includes interactions between the ions and the colloid.

This nomenclature and its interpretation are in agreement with the treatment presented by Overbeek (1956).

An additional point must be made. In certain theories of Donnan systems, the activity of the cations in a suspension is assumed to be the sum of two parts: the activity of the cations associated with the colloid $(Z)$ and the activity of cations associated with the anions in the suspension $(Y)$. Thus,

$$
a_{+}{ }^{I}=Z+Y
$$

This separation does not appear to have meaning. Ionic activities are not additive in the way the concentrations are additive. For instance, if one has a mixture which is $0.1 \mathrm{M}$ in $\mathrm{KCl}$ and $0.1 \mathrm{M}$ in $\mathrm{KNO}_{3}$, the concentrations may be added to find that the solution is $0.2 \mathrm{M}$ in $\mathrm{K}^{+}$. However, the activity of all potassium ions in the mixture is the same.

\section{Electrical potential measurements in Donnan systems}

Consider the following galvanic cell:

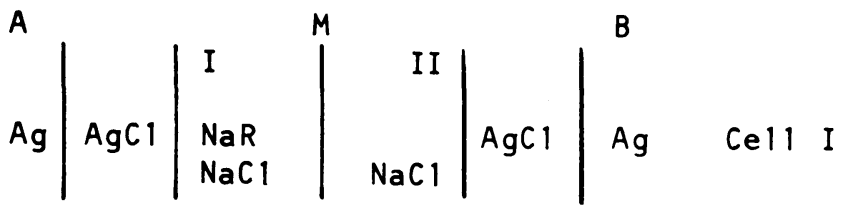

This cell consists of two silver-silver chloride electrodes, each immersed in a phase of a Donnan system. Phase I contains the colloid $\mathrm{NaR}$ and $\mathrm{NaCl}$. Phase II contains $\mathrm{NaCl}$ only. The emf of the cell may be found by the methods used in Section I. The electrode reaction at $A$ is

$$
\mathrm{Ag}+\mathrm{Cl}^{-} \rightarrow \mathrm{AgCl}+e^{-}
$$

By assuming electrochemical equilibrium on open circuit $(\Delta G=0)$, separating the $\bar{G}_{i}$ for charged species, and applying the definition of ionic activity, we find

$$
\Delta \psi_{A}=\psi^{\mathrm{I}}-\psi^{A}=\Delta \psi_{A}^{\circ}+\frac{R T}{F_{y}} \ln a_{\mathrm{Cl}^{-}} \mathrm{I}
$$

where $\psi^{A}$ is the electric potential of the piece of silver at $A$. Similarly at $B$,

$$
\Delta \psi_{B}=\psi^{\mathrm{II}}-\psi^{B}=\Delta \psi^{\circ}{ }_{B}+\frac{R T}{F_{y}} \ln a_{\mathrm{C} 1^{-I I}}
$$


Combining equations (2-23) and (2-24), we obtain

$$
\begin{aligned}
\Delta \psi_{A}-\Delta \psi_{B} & =\left(\psi^{B}-\psi^{A}\right)+\left(\psi^{\mathrm{I}}-\psi^{\mathrm{II}}\right) \\
& =\frac{R T}{F_{y}} \ln \frac{a_{\mathrm{CI}^{-} \mathrm{I}}}{a_{\mathrm{CI}}{ }^{-\mathrm{II}}}
\end{aligned}
$$

Using equation (2-20), we find that

$$
\begin{gathered}
\mathcal{E}=\psi^{B}-\psi^{A} \\
=\Delta \psi_{B}-\Delta \psi_{A}+\Delta \psi_{m}
\end{gathered}
$$

Equation (2-22) for the membrane potential in the present case becomes

$$
\Delta \psi_{m}=-\frac{R T}{F_{y}} \ln \frac{a_{\mathrm{Cl}^{-}}}{a_{\mathrm{C} 1^{-\mathrm{II}}}}
$$

Substituting equation (2-25) and (2-28) in equation (2-27) gives

$$
\varepsilon=0
$$

Thus, by the extrathermodynamic method, we conclude that there is no potential between $\mathrm{Ag}-\mathrm{AgCl}$ electrodes immersed in each phase of a Donnan system, even though $\mu_{\mathrm{Cl}^{-\mathrm{I}}} \neq \mu_{\mathrm{Cl}^{-}}$-II . This fact is often useful experimentally in determining whether or not a suspension has come to equilibrium with its dialyzate.

Consider now a second cell:

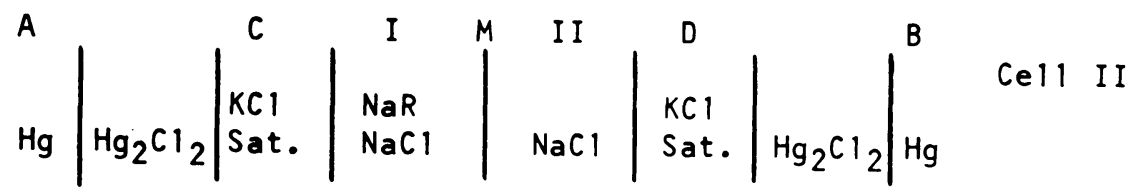

Here we have two calomel half-cells with KCl-saturated salt bridges, each immersed in a phase of the Donnan system. The electrode reaction at $A$ is

$$
2 \mathrm{Hg}+2 \mathrm{Cl}^{-} \rightarrow \mathrm{Hg}_{2} \mathrm{Cl}_{2}+2 e^{-}
$$

The corresponding electrode potential is

$$
\Delta \psi_{A}=\psi^{C}-\psi^{A}=\Delta \psi_{A}^{\circ}+\frac{R T}{F_{y}} \ln a_{\mathrm{C} 1^{-}}{ }^{C}
$$

Similarly at $B$,

$$
\Delta \psi_{B}=\psi^{D}-\psi^{B}=\Delta \psi^{\circ}{ }_{B}+\frac{R T}{F_{y}} \ln a_{\mathrm{C} 1^{-D}}
$$

Therefore,

$$
\begin{aligned}
\Delta \psi_{A}-\Delta \psi_{B} & =\left(\psi^{B}-\psi^{A}\right)-\left(\psi^{D}-\psi^{C}\right) \\
& =\frac{R T}{F_{y}} \ln \frac{a_{\mathrm{Cl} 1^{-}}}{a_{\mathrm{Cl}}{ }^{D}}
\end{aligned}
$$

Now, $a_{\mathrm{Cl}^{-}}{ }^{C}$ and $a_{\mathrm{Cl}^{-}}{ }^{D}$ are the activity of $\mathrm{Cl}^{-}$in two identical saturated $\mathrm{KCl}$ solutions, so that they are equal. Thus, $\Delta \psi_{A}=\Delta \psi_{B}$, and

$$
\psi^{B}-\psi^{A}=\psi^{D}-\psi^{C}
$$


or, since $\psi^{B}-\psi^{A}$ is the cell emf,

$$
\mathcal{E}=\psi^{D}-\psi^{C}
$$

We must examine the interfaces between the bridges and the phases of the Donnan system. The Henderson equation (1-141) predicts that if the concentration of phase $\mathrm{I}$ is low, and if $v_{\mathrm{K}^{+}}=v_{\mathrm{Cl}^{-}}$, then the interface potential between bridge $D$ and phase II should be very small and may be neglected. In many quarters, the same assumption has been made at the interface between bridge $C$ and phase I. If these assumptions are arbitrarily made,

$$
\begin{aligned}
\psi^{\mathrm{I}} & =\psi^{C} \\
\psi^{\mathrm{II}} & =\psi^{D}
\end{aligned}
$$

and equation (2-35) becomes

$$
\begin{aligned}
\mathcal{E} & =\psi^{\mathrm{II}}-\psi^{\mathrm{I}} \\
& =\Delta \psi_{m}
\end{aligned}
$$

Thus, if the assumptions are correct, Cell II should measure the membrane potential. Furthermore,

$$
\Delta \psi_{m}=\frac{R T}{F_{y}} \ln \frac{a_{\mathrm{Cl}^{-}} \mathrm{II}}{a_{\mathrm{C} 1^{-\mathrm{I}}}}=\frac{R T}{F_{y}} \ln \frac{a_{\mathrm{Na}^{+}}}{a_{\mathrm{Na}^{+}}{ }^{+\mathrm{II}}}
$$

Thus, if phase II is very dilute so that $a_{\mathrm{Cl}^{-}}{ }^{\mathrm{II}}$ and $a_{\mathrm{Na}^{+}}{ }^{\mathrm{II}}$ can be found from the DH theory, $a_{\mathrm{Cl}^{-\mathrm{I}}}$ or $a_{\mathrm{Na}^{+}}{ }^{\mathrm{I}}$ can be calculated.

Considerable controversy has arisen over the assumption that the junction potential at the boundary between $C$ and I can be neglected. There can be little doubt that the assumptions underlying the Henderson equation are not applicable to the boundary between the bridge and the suspension. Since there is no means available for measuring junction potential, the significance of measurements made with Cell II, or activities calculated from such measurements, must remain in doubt.

An additional point concerning the interpretation of measurements made with Cell II is of interest. Suppose that the colloid in phase I consists of charged particles surrounded by ions whose concentration gradients are governed by the doublelayer theory to be developed in the next section. The question arises, to what extent does the measured emf in Cell II include the electric potential in the diffuse layer around the particle? A wholly unambiguous answer to this question can be provided.

To make matters clear, consider the following membrane system:

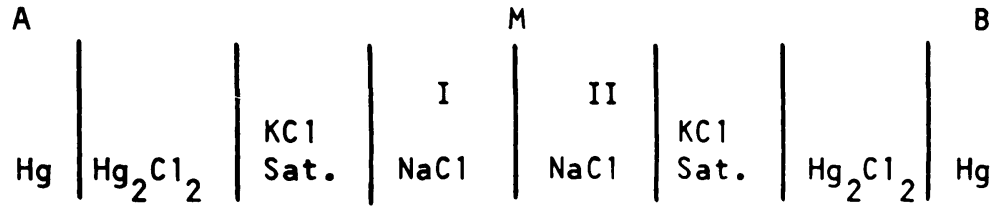

B

Cell III

Here, $M$ is a membrane permeable to $\mathrm{Na}^{+}$ions only, I and II are dilute solutions of $\mathrm{NaCl}$ at different concentrations. At electrochemical equilibrium,

$$
\bar{G}_{\mathrm{Na}^{+}}+\mathrm{I}=\bar{G}_{\mathrm{Na}^{+}}+\mathrm{II}
$$


and

$$
\begin{aligned}
& \mu_{\mathrm{Na}^{+}}{ }^{\mathrm{I}}+F_{y} \psi^{\mathrm{I}}=\mu_{\mathrm{Na}^{+}}{ }^{\mathrm{II}}+F_{y} \psi^{\mathrm{II}} \\
& F_{y}\left(\psi^{\mathrm{II}}-\psi^{\mathrm{I}}\right)=\mu_{\mathrm{Na}^{+}}{ }^{\mathrm{I}}-\mu_{\mathrm{Na}^{+}} \mathrm{II}
\end{aligned}
$$

It is emphasized again that $\psi^{\mathrm{II}}-\psi^{\mathrm{I}}$ is due to slight charge excesses in each phase which are localized at the membrane. Within each phase we have electrostatic interactions, of the type considered in the DH theory, which are included in the $\mu_{i}$ and therefore in $a_{i}$ :

$$
\left(\psi^{\mathrm{II}}-\psi^{\mathrm{I}}\right)=\frac{R T}{F_{y}} \ln \frac{a_{\mathrm{Na}^{+}} \mathrm{I}}{a_{\mathrm{Na}^{+}} \mathrm{II}^{2}}
$$

If the extrathermodynamics is applied to Cell III in the same way that it was applied to Cell II, and if we make the ad hoc assumption that junction potentials at the bridges are absent, then

$$
\mathcal{E}=\psi^{\mathrm{II}}-\psi^{\mathrm{I}}=\Delta \psi_{m}
$$

Thus, under the assumed conditions, the cell measures only the membrane potential, and does not directly measure the difference between the electrostatic interactions with each phase. Indeed, if the solutions are so dilute that the interactions can be entirely neglected, we still have a large membrane potential given as

$$
\mathcal{E}=\frac{R T}{F_{y}} \ln \frac{c_{\mathrm{Na}^{+}}+\mathrm{I}}{c_{\mathrm{Na}^{+}} \mathrm{II}}
$$

where $c$ is the concentration.

Exactly the same considerations apply to Cell II. Whatever the nature of the electrostatic interactions in the colloidal phase, if we make the ad hoc assumption that the junction potentials between the bridges and the phases of the Donnan system are zero, the cell emf is equal to $\Delta \psi_{m}$, which in turn results from a small charge separation at the membrane. At equilibrium, this $\Delta \psi_{m}$ is related to the ionic activities by equation (2-22) and ionic activities include all electrostatic interactions within a phase. In the case of an ideal Donnan system, all interactions can be neglected and the membrane potential is given by equation (2-44).

A related question concerning $\mathrm{pH}$ measurements in suspensions is sometimes raised. Let $\mathrm{pH}$ electrodes be immersed in a suspension of particles with diffuse layers. Assuming no junction potential, to what point in the double layer does the activity or $\mathrm{pH}$ measurement refer? To find the answer, consider the cell:

$$
\begin{array}{c|ccc|c|l} 
& I & & I I & \text { II I } \\
\mathrm{Ag} & \mathrm{AgCl} & \mathrm{Std} & \mathrm{HCl} & \mathrm{HR} & \mathrm{KCl} \\
\mathrm{HCl} & \mathrm{Sat.} & \mathrm{Hg}_{2} \mathrm{Cl}_{2} & \mathrm{Hg} & \mathrm{Ce} 11 \mathrm{IV}
\end{array}
$$

Phase $\mathrm{I}$ is a standard $\mathrm{HCl}$ solution enclosed in a glass electrode. $g$ is a glass membrane permeable to $\mathrm{H}^{+}$ions only. Phase II is a suspension containing $\mathrm{HR}$ and $\mathrm{HCl}$. The application of extrathermodynamics (see Section I), yields

$$
\varepsilon=\varepsilon_{\mathrm{pH}}^{\circ}-\frac{R T}{F_{y}} \ln a_{\mathrm{H}^{+}}{ }^{\mathrm{II}}+\psi^{\mathrm{III}}-\psi^{\mathrm{II}}
$$


If the ad hoc assumption is made that $\psi^{\mathrm{III}}-\psi^{\mathrm{II}}$ is zero, $a_{\mathrm{H}^{+}}{ }^{\mathrm{II}}$ can be calculated from $\varepsilon$. The point deserving emphasis is that $a_{\mathrm{H}+}$ in this equation is defined in terms of the chemical potential which is uniform throughout the phase. Thus, in the absence of junction potential, the cell assembly measures the chemical potential of $\mathrm{H}^{+}$in the phase, and this chemical potential is not a function of position in any of the diffuse layers which may be present in phase II. The question posed above thus has no meaning.

\section{Filtration of a Donnan system}

Perhaps the most common method of extracting a solution from a soil system or suspension is filtration. To apply the Donnan theory to this problem, consider a suspension which is, at some point during the filtration, in equilibrium with a solution phase of infinitesimal volume. For simplicity, the electrolyte is taken as $\mathrm{NaCl}$.

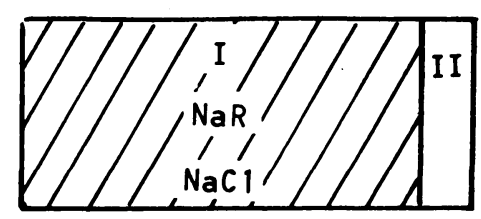

Let the volume of the remaining suspension be $V$ and the amount of salt in the suspension be $a$. The volume of the drop will be $-d V$ and the amount of salt in the drop will be $-d a$. Thus,

$$
m_{\mathrm{Cl}^{-}} \mathrm{II}=\frac{d a}{d V}
$$

If $V_{0}$ is the initial volume and $m_{p}^{\circ}$ the original concentration of the colloid, then the concentration of colloid at a time when the volume is $V$ will be

$$
m_{p}=\frac{V_{0} m_{p}^{\circ}}{V}
$$

Then,

$$
\begin{aligned}
m_{\mathrm{Na}^{+}}{ }^{\mathrm{I}} & =m_{p}+m_{\mathrm{Cl}^{-1}} \\
& =\frac{V_{0} m_{p}}{V}+m_{\mathrm{Cl}^{-}}{ }^{-\mathrm{I}}
\end{aligned}
$$

Applying the equilibrium condition,

$$
\theta^{2}\left(m_{\mathrm{Na}^{+}}{ }^{\mathrm{I}}\right)\left(m_{\mathrm{Cl}^{-}}{ }^{\mathrm{I}}\right)=\left(m_{\mathrm{Cl}^{-}}{ }^{\mathrm{II}}\right)^{2}
$$

Substituting $m_{\mathrm{Cl}^{-}}{ }^{\mathrm{I}}=a / V$ and equation (2-47) in equation (2-48), we derive

$$
m_{\mathrm{Cl} 1^{-} \mathrm{II}}=\frac{\theta}{V} a\left(a+V_{0} m_{p}^{\circ}\right)
$$

Therefore,

$$
\frac{d a}{d V}=\frac{\theta}{V} a\left(a+V_{0} m_{p}^{\circ}\right)
$$

After integrating equation (2-50) and substituting the result into equation (2-49), we obtain

$$
m_{\mathrm{C} 1^{-}} \mathrm{II}=\frac{\theta \sqrt{a^{2}+a V_{0} m_{p}^{\circ}}}{V_{0}\left[\frac{2 a+V_{0} m_{p}^{\circ}+2 a^{2}+a V_{0} m_{p}^{\circ}}{J}\right]}
$$


where $J$ is a constant given as

$$
J=2 a_{0}+V_{0} m_{p}^{\circ}+2 \sqrt{a^{2}{ }_{0}+a_{0} V_{0} m_{p}^{\circ}}
$$

and $a_{0}$ is the initial amount of salt in the suspension. $m_{\mathrm{Cl}^{-}}$-II may now be plotted as a function of $a$ or $V$. This has been done in figures 3 and 4 for the arbitrarily assumed values $m_{p}^{\circ}=V_{0}=a_{0}=1$. In these plots, the filtration proceeds from right to left along the abscissa since $a$ and $V$ decrease.

For $\theta=1$, the initial drop filtrate concentrations are higher than the initial average concentration in the suspension due to the negative adsorption. This effect is decreased by nonideality $(\theta<1)$. At $\theta=0.5$, the initial drop concentration is lower than initial mean concentration, corresponding to the positive anion adsorption discussed above.

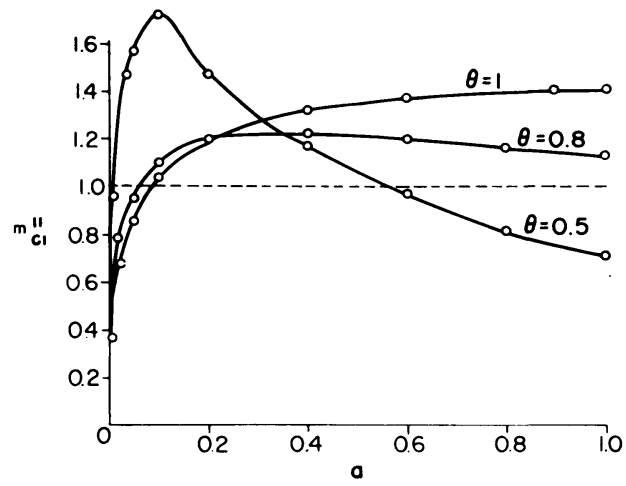

Figure 3. The filtrate concentration $\left(m_{\mathrm{Cl}}{ }^{\mathrm{II}}\right)$ as a function of the salt remaining in the suspension ( $a$ ) during the filtration of a Donnan system at various degrees of ideality.

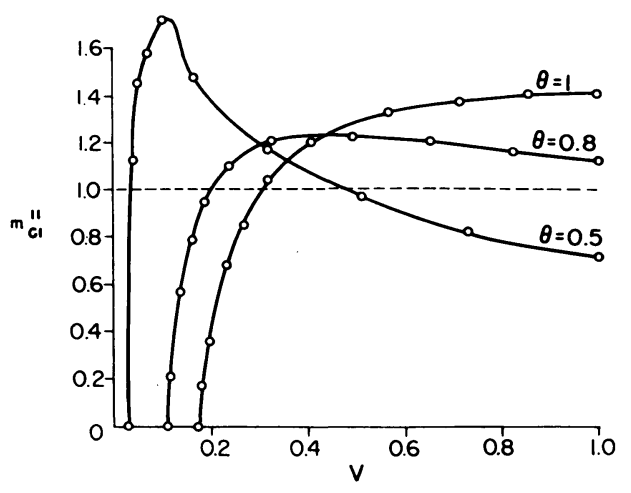

Figure 4. The filtrate concentration $\left(m_{\mathrm{Cl}}{ }^{\mathrm{II}}\right)$ as a function of the volume of the suspension $(V)$ during the filtration of a Donnan system at various degrees of ideality.

It is interesting to note that the concentration curves fall to zero at finite values of the volume. At $\theta=1$, this occurs because salt is removed from the system at a higher concentration than the initial mean for over more than half of the filtration.

The curves in figures 3 and 4 were obtained by integrating equation (2-50) at constant $\theta$. Actually, $\theta$ would be expected to be a function of the salt content of the suspension. The curves therefore have the same arbitrary significance discussed in connection with figures 1 and 2 .

The Donnan theory can be applied to other methods of obtaining extracts from soils. Some of these have been worked out and discussed by the writer (Babcock, 1960).

\section{Osmotic equilibrium}

Thus far, nothing has been said about equilibrium conditions for water in a Donnan system. In effect, the membrane has been made impermeable to water as well as colloid. We now wish to relax this restriction. The treatment parallels the development of the osmotic pressure equations in Section I.

Consider a Donnan system contained in an apparatus which permits the pressure on either phase to be varied by means of pistons: 


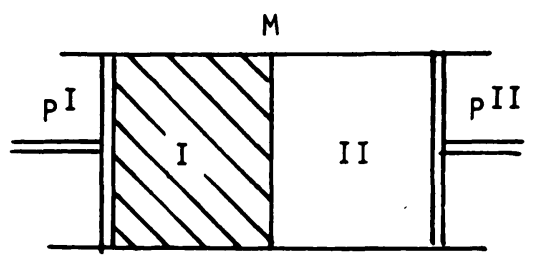

Assume that every diffusible electrolyte in the system is in electrochemical equilibrium and the pressures $P^{\mathrm{II}}$ and $P^{\mathrm{I}}$ are so adjusted that the water is also in equilibrium. By a derivation parallel to equation (1-95), we find (when $P^{\mathrm{II}}=P^{\circ}$ )

$$
P_{\mathrm{eq}}^{\mathrm{I}}-P^{\circ}=-\frac{R T}{\bar{V}_{w}^{\mathrm{I}}} \ln \frac{a_{w}^{\mathrm{I}}}{a_{w}^{\mathrm{II}}}
$$

where $a_{w}{ }^{\mathrm{I}}$ and $a_{w}{ }^{\mathrm{II}}$ are the activities of the water in the phases when the pressure on each phase is $P^{\circ}$, and $P_{\text {eq }}{ }^{\mathrm{I}}$ is the equilibrium pressure. The constancy of $\bar{V}_{w}{ }^{\mathrm{I}}$ is assumed in the derivation. The term $P_{\text {eq }}{ }^{\mathrm{I}}-P^{\circ}$ is not the osmotic pressure of the suspension, which is defined as the pressure necessary to make $\bar{G}_{w}{ }^{\mathrm{I}}$ equal to $\bar{G}^{\circ}{ }_{w}$. In the present case we have made $\bar{G}_{w}^{\text {I }}$ equal to $\bar{G}_{w}^{\text {II }}$ at $P^{\text {II }}=P^{\circ}$.

To find the distribution of ions, we begin by assuming that phase $\mathrm{I}$ is in the state of composition which it has at equilibrium, but the pressure of the phase is $P^{\circ}$. Then

$$
\mu^{p}{ }_{i}-\mu_{i}^{\circ}=R T \ln a_{i}^{p}
$$

where superscript $p$ denotes phase $\mathrm{I}$ at $P^{\mathrm{I}}=P^{\circ}$. If now the pressure is increased to its equilibrium value we find, assuming incompressibility,

$$
\mu_{i}^{\mathrm{I}}-\mu^{p}{ }_{i}=\bar{V}_{i}^{\mathrm{I}}\left(P_{\mathrm{eq}}^{\mathrm{I}}-P^{\circ}\right)
$$

Combining equations (2-54) with (2-55) to eliminate $\mu^{p}{ }_{i}$, we obtain

$$
\mu_{i}^{\mathrm{I}}-\mu_{i}^{\circ}=R T \ln a_{i}^{p}+\bar{V}_{i}^{\mathrm{I}}\left(P_{\mathrm{eq}}^{\mathrm{I}}-P^{\circ}\right)
$$

Substituting $\mu_{i}{ }^{\mathrm{II}}-\mu_{i}^{\circ}=R T \ln a_{i}{ }^{\mathrm{II}}$ and equation (2-56) in the equilibrium condition equation $(2-19)$, we obtain

$$
R T \ln a^{p}{ }_{i}+\bar{V}_{i}{ }^{\mathrm{I}}\left(P_{\mathrm{eq}}{ }^{\mathrm{I}}-P^{\circ}\right)+z_{i} F_{y} \psi^{\mathrm{I}}=R T \ln a_{i}{ }^{\mathrm{II}}+z_{i} F_{y} \psi^{\mathrm{II}}
$$

Thus,

$$
\Delta \psi_{m}=\frac{R T}{z_{i} F_{y}} \ln \frac{a^{p} i}{a_{i}{ }^{\mathrm{II}}}+\frac{\ddot{V}_{i}{ }^{\mathrm{I}}}{z_{i} F_{y}}\left(P_{\mathrm{eq}}{ }^{1}-P^{\circ}\right)
$$

This formula replaces equation (2-22). By a very similar derivation, the macroscopic equation for the distribution of an electrolyte across the membrane can be found:

$$
-R T \ln \frac{a^{p}{ }_{ \pm}}{a_{ \pm}{ }^{\mathrm{II}}}=\frac{\bar{V}^{\mathrm{I}}}{\nu}\left(P_{\mathrm{eq}}^{\mathrm{I}}-P^{\circ}\right)
$$

where $\nu$ is the number of ions into which the electrolyte dissociates.

\section{Applicability to soil systems}

As already remarked, the only limitation which appears to exist in the macroscopic equations is the determination of the mean ionic concentrations in the suspension. In very wet systems, for example, a $1 \%$ bentonite suspension, it seems 
reasonable to write equation (2-9), which states that the average cation concentration is simply the sum of the particle concentration and the anion concentration, each specified in equivalents per liter.

The desirability of this approach is by no means obvious when we consider soil systems at water contents in the field moisture range. As we will see in a later chapter, in such soil systems there is frequently reason to assume that the solution consists of two discrete phases: an exchanger phase and a solution phase. There is nothing formally incorrect in applying equation (2-9) to such cases, but it seems awkward and unnatural. A different sort of model altogether, however, becomes possible. We suppose that the soil system is composed of a discrete phase containing exchangeable cations, and no anions, and a homogeneous solution phase. It does not immediately follow that the filtrate would have a composition identical with this homogeneous solution owing to the possibility of a membrane potential at the filtering interface. This model will be examined in some detail in the chapter on ion exchange.

Negative adsorption of anions in soil systems is well established, but it has not been extensively studied. In clay suspensions it has been studied by Whitney and Peech (1952) and in soil systems at low water content by Bower and Goertzen (1955). The results are not inconsistent with equations (2-16) and (2-17). In general $\alpha$ decreases with salt content while $\beta$ increases. Positive adsorption of anions has been more extensively studied, but nearly always under conditions where it may be the result of positive adsorption sites on clays (for example, kaolinite at low $\mathrm{pH}$ ). Whether positive adsorption can occur as a Donnan effect in a system of negative particles is not known.

Membrane potentials between clays and filtrates, as measured with Cell II, have frequently been made (Davis, 1942; Peech and Scott, 1950; Whitney and Peech, 1952; Peech, Olson, and Bolt, 1953). Jenny et al. (1950), however, state that appreciable junction potentials may occur at a salt bridge in a clay suspension. Recent measurements of apparent mobilities of $\mathrm{K}^{+}$and $\mathrm{Cl}^{-}$in clays tend to confirm this. It appears that nothing conclusive can be inferred from efforts to measure membrane potentials. The significance of the "suspension effect" for soil $\mathrm{pH}$ is likewise open to question (Jenny, et al., 1950; Peech, Olson, and Bolt, 1953).

Few measurements of the osmotic pressure of clay suspensions have been made. Low (1955) obtained interesting results using a dynamic flow method. However, the significance of equation (2-59) does not appear to have been examined.

In summary, the scant data available at the present time do not permit an evaluation of the significance of the Donnan theory for soil systems. 


\section{Section III}

\section{DOUBLE-LAYER THEORY}

\section{GOUY THEORY}

\section{Introduction}

A theory for the distribution of ionic concentrations in the neighborhood of electrostatically charged particles was developed by Gouy in 1910. Since that time the theory has been presented and discussed in many places (Verwey and Overbeek, 1948), and it has been applied to soil systems in various ways (Bolt and Peech, 1953; Bolt, 1955a).

In many applications of the Gouy theory, the charge on the colloidal particles is assumed to arise from the preferential adsorption of one ionic species from a solution phase. Electrostatic neutrality then requires the presence of a second layer of ions of opposite charge. The "double layer" thus consists of two layers of adsorbed ions. For example, such double layers arise in systems of colloidal silver iodide particles in equilibrium with a solution of the ions. The meaning of the expression "double layer" is unambiguous in such cases.

When the Gouy theory is applied to clay particles, the significance of the expression "double layer" may not always be clear. In many clay systems, the charge on the particles is due to isomorphic substitutions in the lattice of the particles, rather than to adsorption on the surface. Such a particle charge requires the presence of a layer of ions of opposite charge on the surfaces. Thus, there is actually only one layer of adsorbed ions, and in this section it will be called the "diffuse layer" since it extends outward from the particle surface.

The Gouy theory as it is usually presented yields only the distribution of concentrations and potentials around the colloidal particles. Once these have been obtained, other problems may be attacked. For example, Verwey and Overbeek (1948) used the distributions in developing a theory of suspension stability. In a later section, the Gouy theory will be used to find the chemical potential of ionic species in suspensions.

\section{Infinite plate distance}

We begin by assuming that the surface of the colloidal particles can be represented by a flat surface without edge effects (that is, an infinite surface). The surface has a continuous and uniform electrostatic charge density. It is immersed in an electrolyte solution which has a uniform dielectric constant. The charge on the surface is neutralized by an excess of ions of opposite sign, and all ions are taken as point charges. The model is completed by assuming that the electric potential is given by the Poisson equation (1-111) and that the ionic concentrations are governed by the Boltzmann equation in the form of equation (1-114). The latter condition means that only electrostatic interactions with the surface are considered, and that the mutual ionic interactions such as those treated by the Debye-Hückel theory are omitted. Thus, we have at the start

$$
\frac{d^{2} \phi}{d x^{2}}=-\frac{4 \pi \rho}{D}
$$


and

$$
n_{i}=n^{\circ}{ }_{i} \exp \left(-\frac{z_{i} \ell \phi}{k T}\right)
$$

Equation (3-1) is the Poisson equation for the electric potential in the $x$ direction measured outward from the surface, and all other symbols have the same meaning as in equations (1-111) and (1-114). The density of charge at any point in the diffuse layer is given as

$$
\rho=\sum z_{i} e n_{i}
$$

Combining equations (3-1), (3-2) and (3-3) leads to an equation similar to that used in the $\mathrm{DH}$ theory:

$$
\frac{d^{2} \phi}{d x^{2}}=-\frac{4 \pi}{D} \sum z_{i} e n^{\circ}{ }_{i} \exp \left(-\frac{z_{i} \ell \phi}{k T}\right)
$$

The Gouy theory is concerned with the solution of equation (3-4) for various special cases since no general solution exists.

We first assume that the suspension contains a single symmetrical electrolyte. Equation (3-4) may then be written

$$
\frac{d^{2} \phi}{d x^{2}}=-\frac{4 \pi\left|z_{i}\right| e n_{i}^{\circ}}{D}\left[\exp \left(-\frac{\left|z_{i}\right| e \phi}{k T}\right)-\exp \left(\frac{\left|z_{i}\right| e \phi}{k T}\right)\right]
$$

where $\left|z_{i}\right|$ is the absolute value of the valence of either ion. It is convenient to introduce a new parameter,

$$
y=\frac{\left|z_{i}\right| e \phi}{k T}
$$

Equation (3-5) then becomes

$$
\frac{d^{2} y}{d x^{2}}=-\frac{4 \pi z^{2} i^{2} n_{i}^{\circ}}{D k T}[\exp (-y)-\exp (y)]
$$

Furthermore, recalling that by definition

$$
\sinh y=\frac{\exp (y)-\exp (-y)}{2}
$$

(where $\sinh y$ is called the hyperbolic sine of $y$ ) we obtain

$$
\frac{d^{2} y}{d x^{2}}=\frac{8 \pi z^{2} e^{2} n^{\circ}}{D k T} \sinh y
$$

It is now convenient to define a new parameter,

Equation (3-8) becomes

$$
\kappa^{2}=\frac{8 \pi z^{2}{ }_{i} e^{2} n^{\circ}}{D k T}
$$

$$
\frac{d^{2} y}{d x^{2}}=\kappa^{2} \sinh y
$$

Applying the integrating factor $2(d y / d x) d x$ to both sides of equation (3-10), the solution of equation (3-10) is found to be

$$
\left(\frac{d y}{d x}\right)^{2}=2 \kappa^{2} \cosh y+C
$$


in which

$$
\cosh y=\frac{\exp (y)+\exp (-y)}{2}
$$

(called the hyperbolic cosine of $y$ ) and $C$ is a constant of integration.

To evaluate $C$, we use the boundary condition that at $x=\infty, y=0$, and $d y / d x=0$. Since $\cosh (0)=1$, we find

$$
\left(\frac{d y}{d x}\right)^{2}=2 \kappa^{2} \cosh y-2 \kappa^{2}
$$

After taking the square root and applying the half angle formula $\sinh (y / 2)=$ $\sqrt{\frac{1}{2}(\cosh y-1)}$, equation (3-12) becomes

$$
\frac{d y}{d x}=-2 \kappa \sinh \left(\frac{y}{2}\right)
$$

since the negative root is required. By integrating equation (3-13), we obtain

$$
\ln \left[\tanh \left(\frac{y}{4}\right)\right]=-\kappa x+C
$$

in which $\tanh y=(\sinh y / \cosh y)$ and $C$ is a constant of integration. $C$ is evaluated by using $y=y_{0}$ at $x=0$. Thus,

$$
\text { in }\left[\frac{\tanh \left(\frac{y}{4}\right)}{\tanh \left(\frac{y_{0}}{4}\right)}\right]=-\kappa x
$$

This can be solved for $y$ as follows:

Let

$$
\tanh \left(\frac{y}{4}\right)=\tanh \left(\frac{y_{0}}{4}\right) \exp (-\kappa x)
$$

$$
a=\tanh \left(\frac{y_{0}}{4}\right)
$$

so that

$$
\begin{aligned}
& \tanh \left(\frac{y}{4}\right)=a \exp (-\kappa x) \\
& y=4 \tanh ^{-1}[a \exp (-\kappa x)]
\end{aligned}
$$

Using an identity for $\tanh ^{-1}$, this becomes

$$
y=2 \ln \left(\frac{1+a e^{-\kappa x}}{1-a e^{-\kappa x}}\right)
$$

Equation (3-17) may also be written

$$
y=2 \ln \left(\frac{e^{x x}+a}{e^{x x}-a}\right)
$$


Knowing $y$ as a function of $x$, one may return to the Boltzmann equation and find the concentration as a function of $x$ as follows:

$$
\begin{gathered}
n_{i}=n^{\circ}{ }_{i} \exp \left(-\frac{z_{i} \ell \phi}{k T}\right) \\
=n^{\circ}{ }_{i} \exp \left(-\frac{z_{i}}{\left|z_{i}\right|} y\right) \\
=n^{\circ}{ }_{i} \exp \left[-\frac{2 z_{i}}{\left|z_{i}\right|} \ln \left(\frac{1+a e^{-\kappa x}}{1-a e^{-\kappa x}}\right)\right] \\
n_{i}=n^{\circ}{ }_{i}\left(\frac{1-a e^{-\kappa x}}{1+a e^{-\kappa x}}\right)^{2 z_{i} /\left|z_{i}\right|}
\end{gathered}
$$

The distribution of the electric potential and the concentration have now been found. We next find the net space charge on the diffuse layer, $\sigma$, by integrating the charge density at each point over the distance:

$$
\sigma=\int_{0}^{\infty} \rho d x
$$

This space charge will be equal to but opposite in sign from the surface charge density. Solving the Poisson equation (3-1) for $\rho$ and substituting in equation $(3-20)$, we find that

$$
\sigma=-\int_{0}^{\infty} \frac{D}{4 \pi} \frac{d^{2} \phi}{d x^{2}} d x
$$

This integrates into

$$
\sigma=-\left.\frac{D}{4 \pi} \frac{d \phi}{d x}\right|_{0} ^{\infty}
$$

Since $d \phi / d x=0$ at $x=\infty$,

$$
\begin{aligned}
\sigma & =\frac{D}{4 \pi}\left(\frac{d \phi}{d x}\right)_{x=0} \\
& =\frac{D k T}{4 \pi\left|z_{i}\right| e}\left(\frac{d y}{d x}\right)_{x=0}
\end{aligned}
$$

Substituting equation (3-13) in equation (3-23), we obtain

$$
\sigma=-\frac{D k T \kappa}{2 \pi\left|z_{i}\right| e} \sinh \left(\frac{y_{0}}{2}\right)
$$

Another form of this equation is

$$
\sigma=-\frac{4 \pi e\left|z_{i}\right| n^{\circ}}{\kappa} \sinh \left(\frac{y_{0}}{2}\right)
$$

The form of these equations becomes simpler if the DH approximation $z e \phi_{0} / k T$ $\ll 1$ is applied. This is equivalent to $y_{0} \ll 1$. Expanding the exponents in equation $(3-7)$, we obtain

$$
\begin{aligned}
\frac{d^{2} y}{d x^{2}} & =\frac{8 \pi z^{2}{ }_{i} e^{2} n^{\circ}}{D k T} y \\
& =\kappa^{2} y
\end{aligned}
$$

Integrating twice and using the same boundary conditions the result is

$$
y=y_{0} \exp (-\kappa x)
$$


Expanding the exponent in the Boltzmann equation and substituting equation (3-27), we obtain

$$
\begin{aligned}
n_{i} & =n^{\circ}{ }_{i}\left[1-\frac{z_{i}}{\left|z_{i}\right|} y\right] \\
& =n^{\circ}{ }_{i}\left[1-\frac{z_{i} y_{0}}{\left|z_{i}\right|} \exp (-\kappa x)\right]
\end{aligned}
$$

Equations (3-27) and (3-29) are limiting laws which apply at low-charge densities. In figure 5, the electric potential is plotted as a function of distance for $\sigma=$ $100 \mathrm{esu} / \mathrm{cm}^{2}$ at two values of $m^{\circ}{ }_{i}$ (the molality at $x=\infty$ ). In figure 6 , the surface electric potential is plotted as a function of $m^{\circ}{ }_{i}$ at $\sigma=100$. It will be noted that

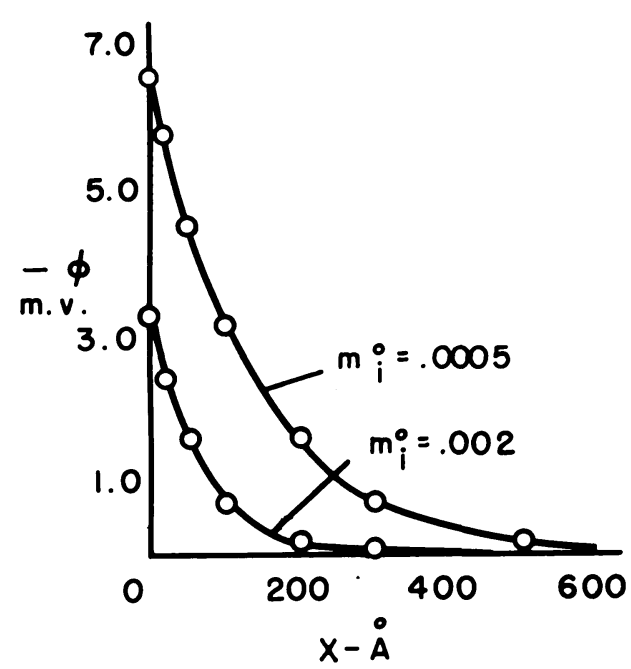

Figure 5. The electric potential in the diffuse layer as a function of distance at two salt concentrations, according to simplified theory.

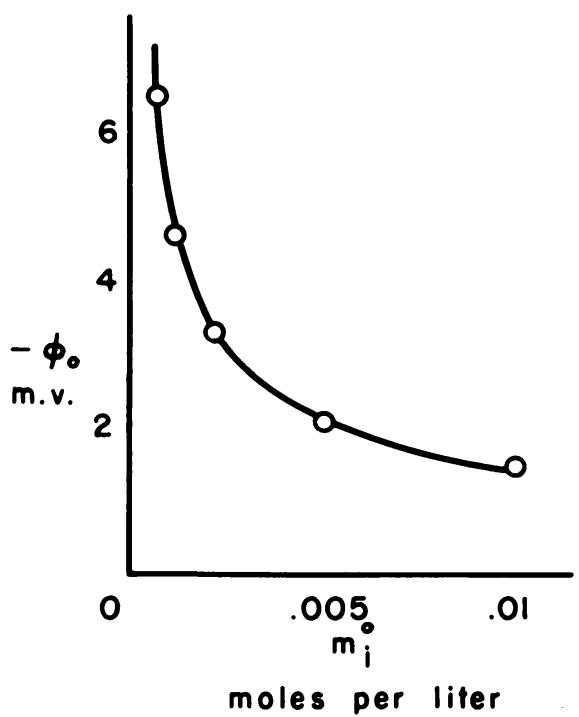

Figure 6. The surface electric potential as a function of salt concentration according to simplified theory.

$\phi_{0}$ increases as $m^{\circ}{ }_{i}$ decreases. This means that to use the limiting equations, $m^{\circ}{ }_{i}$ must be sufficiently high to keep the surface potential low enough to satisfy the condition $y_{0} \ll 1$. At $\sigma=100$, the required concentration is in the order of 0.2 me/liter. It is frequently convenient to define the "double-layer distance" as the distance at which $\phi$ has dropped to $1 / e$ of its value at the surface. From equation $(3-27)$ this is seen to be a distance equal to $1 / \kappa$. Figure 5 shows that this distance decreases as $m^{\circ}{ }_{i}$ increases. In order to obtain calculations which are reasonable, $1 / \kappa$ should be large in relation to actual ionic sizes, and this consideration forms an upper limit for the value of $m^{\circ}$. At $\sigma=100$, this is about $2 \mathrm{me} / \mathrm{liter}$. The limiting equation thus has a narrow range of applicability, and this range decreases as $\sigma$ increases. For clay particles, with $\sigma$ in the order of $10^{4} \mathrm{esu} / \mathrm{cm}^{2}$ or higher, there is no range of applicability.

Using the general equations (3-17) and (3-19), no lower limit on $m^{\circ}{ }_{i}$ is formed. However, it is still desirable to keep $m^{\circ}{ }_{i}$ sufficiently low that $1 / \kappa$ will be large enough to render the assumption of point charges reasonable. Unfortunately, this also 
requires the use of values which are below the values found for clays. At $\sigma=10^{4}$, the value of $1 / \kappa$ required is less than an order of magnitude greater than actual hydrated ion sizes at all reasonable values of $m^{\circ}$. Thus, equations (3-17) and (3-19) will represent only systems with low surface charge densities. In figure 7 , the electric potential is plotted as a function of distance for $m^{\circ}{ }_{i}=.005 M$ and $\sigma=5 \times$ $10^{3} \mathrm{esu} / \mathrm{cm}^{2}$. In figure 8 , the concentrations of cation and anion are plotted for two values of $m^{\circ}{ }_{i}$.

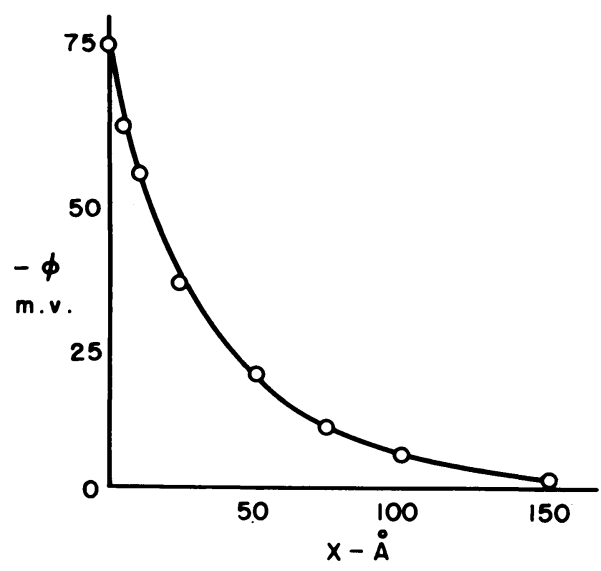

Figure 7. The surface electric potential as a function of distance for $m^{\circ}{ }_{i}=.005 \mathrm{M}$ and $\sigma=$ $5 \times 10^{3}$ esu per sq $\mathrm{cm}$, according to general theory.

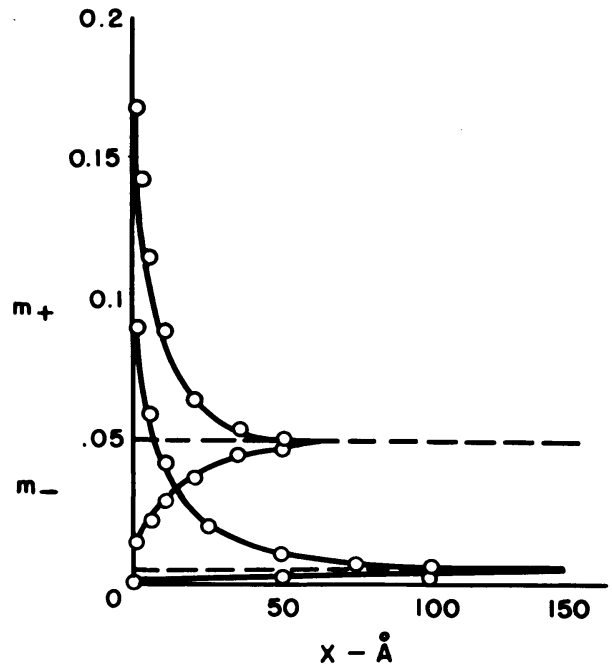

Figure 8. Cation and anion concentrations as a function of distance for $\sigma=5 \times 10^{3}$ esu per sq $\mathrm{cm}$ at $m^{\circ}{ }_{i}=.05$ and $m^{\circ}{ }_{i}=.01$ according to general theory.

It is of interest to recall that the Boltzmann equation in the form of equation (3-2) requires not only that $n^{\circ}{ }_{i}$ be the concentration at $x=\infty$, but also that it represent the mean concentration in the system. We now verify that equation (3-19) meets this requirement. The mean concentration in the diffuse layer is given as

For the cations we find that

$$
\bar{n}_{i}=\frac{\int n_{i} d x}{\int d x}
$$

$$
n_{+}=n_{+}^{\circ}\left(\frac{1-a e^{-\kappa x}}{1+a e^{-\kappa x}}\right)^{2}
$$

Substituting equation (3-31) in equation (3-30) and integrating to find the mean value of $n_{+}$between any two distances $x_{1}$ and $x_{2}$, we obtain

$$
\begin{aligned}
\bar{n}_{+} & =\frac{\int_{x_{1}}^{x_{2}} n^{\circ}\left(\frac{1-a e^{-\kappa x}}{1+a e^{-\kappa x}}\right)^{2}}{x_{2}-x_{1}} d x \\
& =\left.\frac{n^{\circ}}{\kappa\left(x_{2}-x_{1}\right)}\left[\frac{4 a}{e^{\kappa x}+a}+\kappa x\right]\right|_{x_{1}} ^{x_{2}}
\end{aligned}
$$




$$
=\frac{n^{\circ}}{\kappa}\left[\frac{4 a}{\left(x_{2}-x_{1}\right)\left(e^{\kappa x_{2}}+a\right)}-\frac{4 a}{\left(x_{2}-x_{1}\right)\left(e^{\kappa x_{1}}+a\right)}+\kappa\right]
$$

On letting $x_{1} \rightarrow 0$ and $x_{2} \rightarrow \infty$, we find that

$$
\bar{n}_{+}=n_{+}^{\circ}
$$

The same result is obtained for anions. Thus, notwithstanding the concentration gradients near the surface, the average concentrations are equal to $n^{\circ}{ }_{i}$ and the requirement of the Boltzmann equation is satisfied. This result might have been anticipated since at infinite distance between the plates, the colloidal particles are at infinite dilution.

The important findings of the theory in this section are:

a) that in the case of a negative surface, the excess cations near the surface neutralize more of the charge than the anion deficit;

b) that the surface electric potential decreases as the salt concentration increases;

c) that the double-layer distance $1 / \kappa$ decreases as the salt concentration increases. The two most important limitations on the theory are:

a) it applies only to infinitely dilute clay suspensions;

b) it applies only to low surface charge densities.

\section{Finite plate distance}

When the distance between two plates becomes finite, the electric potential between the plates is everywhere finite. The two diffuse layers are then said to intermingle or overlap. We cannot then apply the Boltzmann equation in the form of equation (3-2). By applying statistical mechanics, however, new distribution equations can be developed. We shall attempt a much simpler treatment based on extrathermodynamics.

In the nomenclature adopted in Section II, the chemical potential of all ions in the diffuse layer is constant throughout at equilibrium:

$$
d \mu_{i}=0
$$

By analogy with the treatment of electrochemical systems, we assume that the chemical potential of an ion in a volume element of the diffuse layer is the sum of a concentration term and an electrical term whose source is external to the volume element. Thus, still leaving mutual ionic interactions out of account, we write

$$
d \mu_{i}=k T d \ln n_{i}+z_{i} e d \phi=0
$$

Integrating from any point in the diffuse layer to $x=d$, the distance to the midpoint between the plates, we find

$$
\begin{array}{r}
k T \ln \frac{n_{i}}{n^{d}}+z_{i} e\left(\phi-\phi_{d}\right)=0 \\
n_{i}=n^{d}{ }_{i} \exp \left[-\frac{z_{i} e}{k T}\left(\phi-\phi_{d}\right)\right]
\end{array}
$$

in which $n^{d}{ }_{i}$ and $\phi_{d}$ are values at $x=d$. This equation must be used in place of equation (3-2). 
We next recognize the existence of a purely formal number defined by putting $\phi=0$ :

Therefore,

$$
n_{i}^{\circ}=n_{i}^{d} \exp \left(\frac{z_{i} e \phi_{d}}{k T}\right)
$$

$$
n^{d}{ }_{i}=n^{\circ}{ }_{i} \exp \left(-\frac{z_{i} e \phi_{d}}{k T}\right)
$$

Substituting equation (3-41) in equation (3-39), we obtain

$$
n_{i}=n_{i}^{\circ} \exp \left(-\frac{z_{i} \ell \phi}{k T}\right)
$$

This has exactly the same form as the Boltzmann equation for $d=\infty$, and combination with the Poisson equation leads once more to equation (3-11) for symmetrical electrolytes. However, $n^{\circ}{ }_{i}$ now apparently stands for a hypothetical concentration outside the plates. To evaluate the integration constant in equation (3-11), we use the boundary condition that at $x=d, y=y_{d}$, and $d y / d x=0$. The result is

Thus,

$$
\left(\frac{d y}{d x}\right)^{2}=2 \kappa^{2} \cosh y-2 \kappa^{2} \cosh y_{d}
$$

$$
\frac{d y}{\sqrt{2 \cosh y-2 \cosh y_{d}}}=-\kappa d x
$$

Unfortunately, no solution for this equation exists. However, it can be transformed into the elliptical integral (Verwey and Overbeek, 1948)

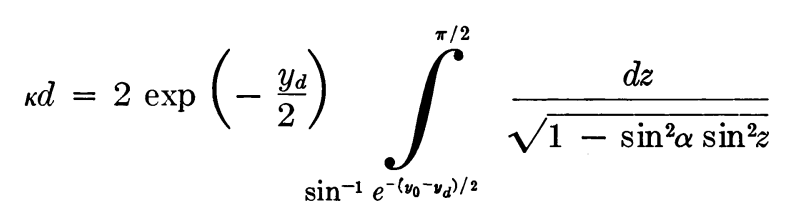

where

$$
\sin \alpha=\exp \left(-y_{d}\right)
$$

and

$$
\sin \alpha \sin ^{2} z=\exp (-y)
$$

Thus, solutions for equation (3-44) can be found from tables.

An expression for the space charge is readily found using equation (3-22). From equation (3-43), we find that

$$
\left(\frac{d y}{d x}\right)_{x=0}=\sqrt{2 \kappa^{2} \cosh y_{0}-2 \kappa^{2} \cosh y_{d}}
$$

and substituting these terms in equation (3-22), we obtain

$$
\sigma=\frac{D k T_{\kappa}}{4 \pi\left|z_{i}\right| e} \sqrt{2 \cosh y_{0}-2 \cosh y_{d}}
$$

This equation can also be put in the form

$$
\sigma^{2}=\frac{D k T n^{\circ}}{\pi}\left(\cosh y_{0}-\cosh y_{d}\right)
$$


An analytical solution for the distribution equations can be found by setting the condition $y_{0} \ll 1$ and expanding exponents. We again obtain equation (3-26) and, after the first integration,

$$
\left(\frac{d y}{d x}\right)^{2}=\kappa^{2} y^{2}-\kappa^{2} y^{2}
$$

Thus,

$$
\frac{d y}{\sqrt{y^{2}-y^{2}}}=-\kappa d x
$$

Integrating from $d$ to $x$, we find that

$$
y=y_{d} \cosh (\kappa d-\kappa x)
$$

Equation (3-22) may again be used to find the space charge. The result can be put in the form

$$
\sigma=-\frac{\phi_{0} \kappa D}{4 \pi} \tanh (\kappa d)
$$

On substituting equation (3-51) in the expanded Boltzmann equation, we obtain the concentrations as functions of distance:

$$
n_{i}=n^{\circ}\left[1-\frac{z_{i} e \phi_{d}}{k T} \cosh (\kappa d-\kappa x)\right]
$$

Equation (3-30) can now be used to find the mean concentration by integrating from $x=0$ to $d$. The result can be put in the form

$$
\bar{n}_{i}=n_{i}^{\circ}\left[1+\frac{4 \pi \sigma z_{i} e}{\kappa^{2} k T D d}\right]
$$

In figure 9, the electric potential is plotted as a function of distance for $\sigma=$ $100 \mathrm{esu} / \mathrm{cm}^{2}$ and $m_{i}^{\circ}=.0005 \mathrm{M}$, as calculated from these limiting laws. In figure 10, the concentrations are plotted, and the mean concentrations are also shown.

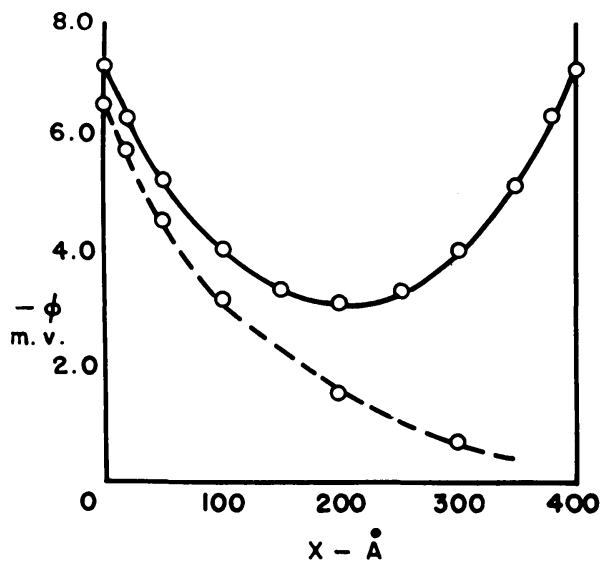

Figure 9. The electric potential as a function of distance for a finite plate distance of $400 \AA$ and $\sigma=100$ esu per sq cm according to simplified theory. $m^{\circ}{ }_{i}=.0005 \mathrm{M}$. Infinite plate distance (dashed line) is included for comparison.

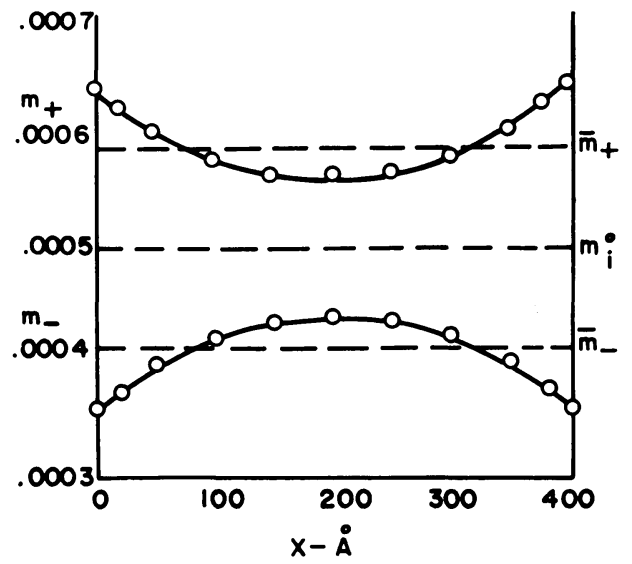

Figure 10. The cation and anion concentrations as a function of distance for a finite plate distance of $400 \AA$ and $\sigma=100 \mathrm{esu}$ per sq $\mathrm{cm}$ according to simplified theory. The mean concentrations are shown. 


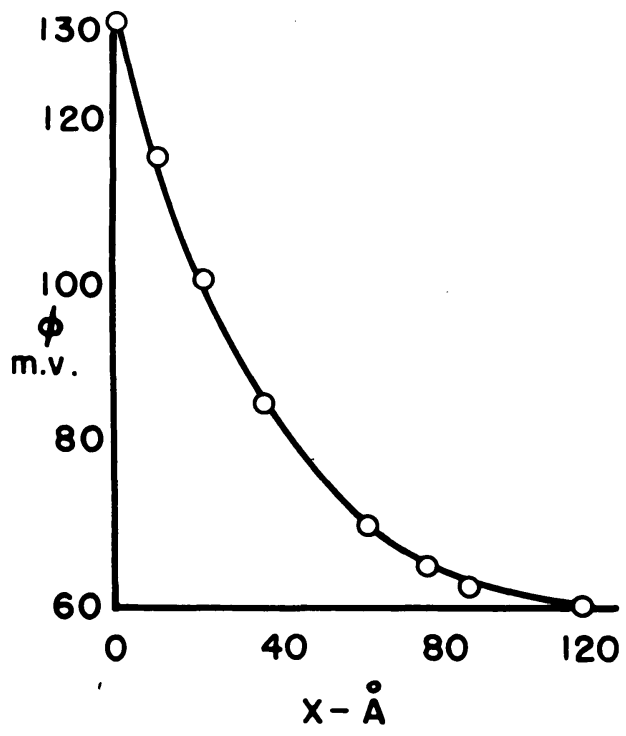

Figure 11. The electric potential as a function of distance for a finite plate distance according to general theory. $\sigma=5 \times 10^{3}$ esu per $\mathrm{sq} \mathrm{cm}$ and $\mathrm{m}^{\circ}=.0005 \mathrm{M}$.

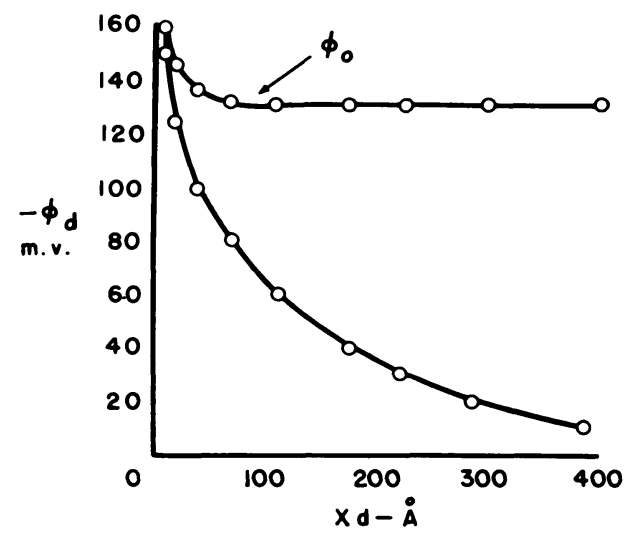

Figure 13. Potential at the midpoint and potential at the surface as functions of the plate distance according to general theory. $\sigma=$ $5 \times 10^{3}$ esu per sq $\mathrm{cm}$ and $m^{\circ}=.0005 \mathrm{M}$.
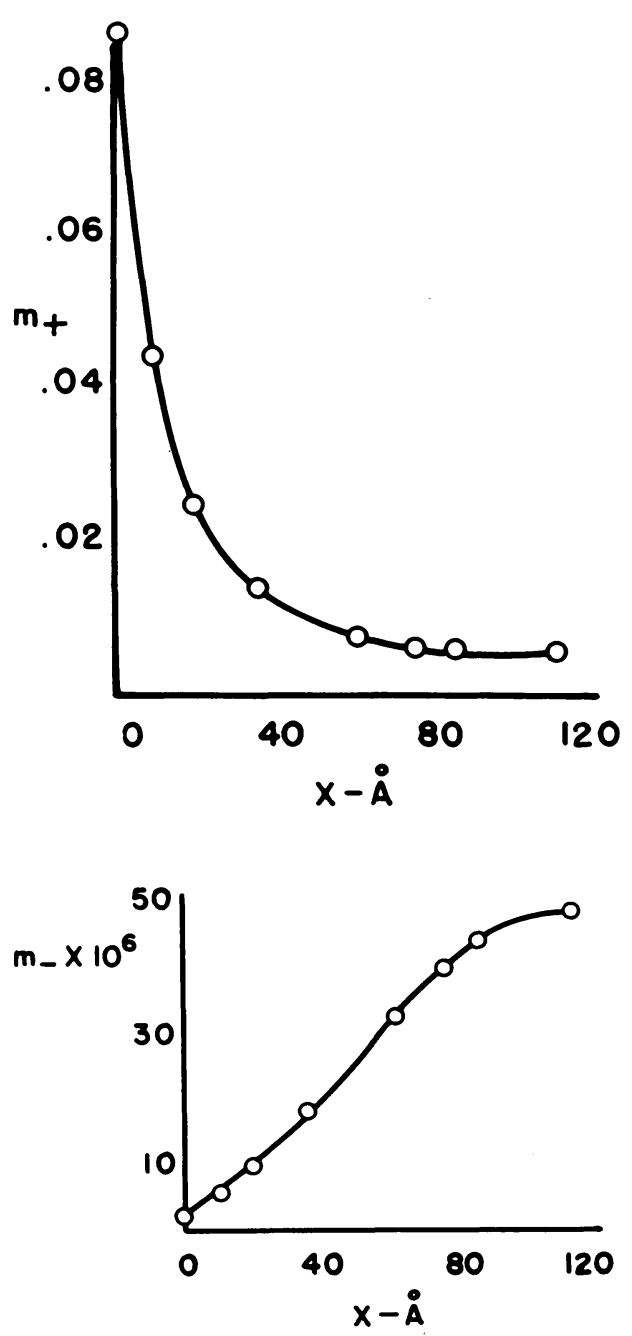

Figure 12. Cation concentrations (top) and anion concentrations (bottom) as a function of distance for finite plate distance according to general theory. $\sigma=5 \times 10^{3}$ esu per sq $\mathrm{cm}$ and $m^{\circ}=.0005 \mathrm{M}$.

The difference between these mean concentrations is of course the clay concentration in me/liter.

Returning to the general case, the electric potential and concentrations are plotted in figures 11 and 12 for $\sigma=5 \times 10^{3} \mathrm{esu} / \mathrm{cm}^{2}, m^{\circ}{ }_{i}=.0005$ and $\phi_{d}=-60 \mathrm{mv}$. In figure $13, \phi_{0}$ and $\phi_{d}$ are plotted as a function of $d$. While $\phi_{d}$ varies widely, $\phi_{0}$ is a constant until $d$ becomes very small. 


\section{CHEMICAL POTENTIALS}

\section{Introduction}

Thus far we have obtained only the expressions for the electric potential and concentrations around the charged colloidal particle. The next task is to calculate the chemical potential of ions in the diffuse layer. As in the Debye-Hückel theory, this will lead to the activity coefficient.

Efforts have been made in a number of places (for example, see Bolt, 1955b) to include mutual ionic interactions in the double-layer theory and to calculate activity coefficients defined in terms of these interactions. However, in the present section, the aim is to calculate the chemical potential defined so as to include surfaceion interactions and to find the corresponding activity coefficients from the Gouy distributions. Mutual ionic interactions are left out of this model on the assumption that surface-ion interactions constitute the first-order effect.

We must first inquire somewhat more closely into the significance of the activity coefficient. Following the usage developed in Section II, we obtain

$$
\begin{aligned}
\mu_{i}-\mu_{i}^{\circ} & =R T \ln a_{i} \\
& =R T \ln \gamma_{i} \bar{m}_{i}
\end{aligned}
$$

in which $a_{i}$ is uniform throughout the phase and $\bar{m}_{i}$ is the mean concentration. We may therefore interpret $\gamma_{i}$ as follows. Imagine the transfer of a chemical species from its standard state to a hypothetical solution which is at molality $\bar{m}_{i}$, which is ideal, and in which $\mu_{i}=\mu^{*}{ }_{i}$. Then,

$$
\Delta \mu_{1}=\mu^{*}{ }_{i}-\mu_{i}^{\circ}=R T \ln \bar{m}_{i}
$$

Next imagine the transfer of the species from the hypothetical ideal solution to a final solution at molality $\bar{m}_{i}$. Then,

The overall $\Delta \mu$ is

$$
\Delta \mu_{2}=\mu_{i}-\mu_{i}^{*}
$$

$$
\Delta \mu_{i}=\Delta \mu_{1}+\Delta \mu_{2}=\mu_{i}-\mu_{i}^{\circ}
$$

From these equations we find

$$
\mu_{i}-\mu^{*}{ }_{i}=R T \ln \gamma_{i}=\Delta \mu_{2}
$$

Thus, to find $\gamma_{i}$ we must find the $\Delta \mu$ for transfer from a hypothetical ideal state at a concentration $\bar{m}_{i}$ to the actual solution at the same concentration. In the DH theory, we realize that if the hypothetical state is to be ideal, the species must be uncharged, so that $\Delta \mu_{2}$ can be found by calculating the reversible work to charge the ions. We may note in passing that in general it is not obvious whether $\Delta \mu_{2}$ is negative or positive. In the $\mathrm{DH}$ theory it is negative because the attraction between ions of opposite signs outweighs the repulsion between ions of like signs.

An alternative to this interpretation is to find the $\Delta G$ for changing the whole system from the hypothetical ideal state to the actual state, and then differentiating with respect to $n_{i}$ to find $\Delta \mu_{2}$ and $\gamma_{i}$.

This latter technique is most advantageous in dealing with colloids. We imagine the colloidal plate and the ions to be in a hypothetical ideal state with concentrations that are uniform and equal to the mean concentrations in the final state. We then find the $\Delta G$ to change the system to its final state and differentiate with respect to $n_{i}$. This leads to $\Delta \mu_{2}$ and to $\gamma_{i}$. 


\section{Charging processes}

First, the electrostatic work required to charge the system must be found. An equation well suited to this problem has been developed by Verwey and Overbeek (1948). The derivation is as follows.

The charge on each ion in the system is $z_{i} e$. We may therefore write the process by which the ions are charged by expressing the change in the charge by $z_{i} e d \lambda$, where $\lambda$ is the fractional part of the charge and will increase from zero to one. The change in the charge on a volume element $d V$, due to the change in the charge of the $i$ th ion species at a concentration $n_{i}$, is the product of the number of ions in the volume $\left(n_{i} d V\right)$ and the change in charge per ion:

$$
d q_{i}=z_{i} e n_{i} d \lambda d V
$$

where $d q_{i}$ is the change in charge. The net change in charge due to all ion species is

$$
d q=\sum z_{i} e n_{i} d \lambda d V
$$

At any point during the charging process the charge on each ion is $z_{i} e \lambda$, so that the charge density $\left(\rho^{\prime}\right)$ of a volume element is given as

$$
\rho^{\prime}=\sum z_{i} e n_{i} \lambda
$$

It follows from equations (3-62) and (3-63) that

$$
d q=\frac{\rho^{\prime}}{\lambda} d \lambda d V
$$

If $\phi^{\prime}$ is the electric potential of the volume element at some point during the charging, then the free energy change is given as

Thus,

$$
\begin{aligned}
d G(e l) & =\phi^{\prime} d q \\
& =\frac{\phi^{\prime} \rho^{\prime}}{\lambda} d \lambda d V
\end{aligned}
$$

$$
\Delta G(e l)=\iint \frac{\phi^{\prime} \rho^{\prime}}{\lambda} d \lambda d V
$$

Since $\lambda$ is independent of position,

$$
\Delta G(e l)=\int \frac{d \lambda}{\lambda} \int \phi^{\prime} \rho^{\prime} d V
$$

Since $\phi^{\prime}$ and $\rho^{\prime}$ vary in the $x$ direction only,

$$
\Delta G(e l)=\int \frac{d \lambda}{\lambda} \int \phi^{\prime} \rho^{\prime} d x
$$

in which $\Delta G(e l)$ is now the free energy change per square centimeter of surface. That is, $\Delta G(e l)$ is the free energy required to charge all volume elements opposite one square centimeter of surface.

One method of employing equation (3-69) immediately suggests itself. We imagine that as $\lambda$ increases, electrochemical equilibrium is continuously maintained as required by the Boltzmann equation. Thus, as the charging goes on, the concentration gradients will be created automatically. To attempt this, consider 
the simplest case in which $y_{0} \ll 1$ and $d=\infty$. At any point during the charging we find from equation (3-27) that

$$
\phi^{\prime}=\phi_{0}{ }^{\prime} \exp \left(-\kappa^{\prime} x\right)
$$

Furthermore, if we replace $\left(z_{i} e\right)^{2}$ in equation (3-9) by $\left(z_{i} e \lambda\right)^{2}$, we find that

Therefore,

$$
\kappa^{\prime}=\lambda \kappa
$$

$$
\phi^{\prime}=\phi_{0}{ }^{\prime} \exp (-\kappa \lambda x)
$$

From the Poisson equation we obtain for the charge density

$$
\begin{aligned}
\rho^{\prime} & =-\frac{D}{4 \pi} \frac{d^{2} \phi^{\prime}}{d x^{2}} \\
& =-\frac{D}{4 \pi}\left(\kappa^{\prime}\right)^{2} \phi^{\prime} \\
& =-\frac{D}{4 \pi} \lambda^{2} \kappa^{2} \phi^{\prime}
\end{aligned}
$$

Thus, at any point in the double layer we do not find $\rho^{\prime}=\lambda \rho$ since the charge and concentration of each volume element are both changing. However, we must obtain

$$
\sigma^{\prime}=\lambda \sigma
$$

since the total number of ions in the diffuse layer does not change. The relation between the surface potential and the space charge for the present case is

Therefore,

$$
\sigma=-\frac{D_{\kappa}}{4 \pi} \phi_{0}
$$

$$
\sigma^{\prime}=-\frac{D \kappa \lambda}{4 \pi}{\phi_{0}}^{\prime}=\lambda \sigma
$$

From equation (3-78) we find

$$
\phi_{0}{ }^{\prime}=\frac{4 \pi \sigma}{D \kappa}=\phi_{0}
$$

Hence, the charging must be at constant surface potential. From equation (3-72) we note that this requires that $\phi^{\prime}=\phi_{0}$ everywhere at $\lambda=0$. The conclusion is that while the electrical work can be found for the equilibrium charging process, this is not the desired process since we wish to begin from an initial state in which the potential is everywhere zero.

To overcome this difficulty, the charging process is carried out with all ionic concentrations fixed at their final values in the diffuse layer. This may be called charging in situ. Later we will find the purely osmotic work to create the concentration gradients and thus obtain the overall $\Delta G$. (An analogous osmotic term does not enter the DH theory since the potential due to the ion atmosphere alone is independent of distance, and does not create concentration gradients.)

For a charging process in situ, only the ionic charges in a volume element change since the concentrations are constant. Thus,

$$
\rho^{\prime}=\lambda \rho
$$


The Boltzmann equation cannot be applied during the charging process as we do not have electrochemical equilibrium. However, the Poisson equation is still applicable. Therefore,

$$
\begin{aligned}
\frac{d^{2} \phi^{\prime}}{d x^{2}} & =-\frac{4 \pi \rho^{\prime}}{D} \\
& =-\frac{4 \lambda \rho}{D} \\
& =\lambda \frac{d^{2} \phi}{d x^{2}}
\end{aligned}
$$

Integration immediately yields

$$
\phi^{\prime}=\lambda \phi
$$

Thus, $\phi^{\prime}$ is everywhere proportional to $\lambda$ and we have the correct initial and final states.

The charging process may now be carried out for the general case with $d=\infty$. Beginning with

$$
\begin{aligned}
\rho^{\prime} & =\lambda \rho \\
& =\lambda \sum z_{i} e n_{i}
\end{aligned}
$$

we substitute equation (3-19) and find that

$$
\rho^{\prime}=\lambda \sum z_{i} e n_{i}^{\circ}\left(\frac{1-a e^{-\kappa x}}{1+a e^{-\kappa x}}\right)^{2 z_{i} /\left|z_{i}\right|}
$$

For a single symmetrical electrolyte,

$$
\rho^{\prime}=\lambda\left|z_{i}\right| e n^{\circ}\left[\left(\frac{1-a e^{-\kappa x}}{1+a e^{-\kappa x}}\right)^{2}-\left(\frac{1-a e^{-\kappa x}}{1+a e^{-\kappa x}}\right)^{-2}\right]
$$

Substituting equation (3-17) in equation (3-83), we obtain

$$
\phi^{\prime}=\frac{2 k T}{|z| e} \ln \left(\frac{1+a e^{-\kappa x}}{1-a e^{-\kappa x}}\right)
$$

Letting $u=\exp (\kappa x)$ and using equations (3-86) and (3-87), we find

$$
\rho^{\prime} \phi^{\prime} d x=\frac{2 k T \lambda^{2} n^{\circ}{ }_{i}}{\kappa}\left[\left(\frac{u-a}{u+a}\right)^{2}-\left(\frac{u+a}{u-a}\right)^{2}\right] \ln \left(\frac{u+a}{u-a}\right) \frac{d u}{u}
$$

After a lengthy integration, we obtain

$$
\begin{aligned}
\int_{0}^{\infty} \rho^{\prime} \phi^{\prime} d x & =\left.\frac{2 k T \lambda^{2} n^{\circ}{ }_{i}}{\kappa}\left[\frac{8 a u}{u^{2}-a^{2}} \ln \left(\frac{u+a}{u-a}\right)-\frac{8 a^{2}}{u^{2}-a^{2}}\right]\right|_{1} ^{\infty} \\
& =-\frac{2 k T \lambda^{2} n^{\circ}{ }_{i}}{\kappa}\left[\frac{8 a^{2}}{1-a^{2}} \ln \left(\frac{1+a}{1-a}\right)-\frac{8 a^{2}}{1-a^{2}}\right]
\end{aligned}
$$

The free energy is now found from equation (3-69):

$$
\Delta G(e l)=\int \frac{d \lambda}{\lambda} \int \rho^{\prime} \phi^{\prime} d x=-\frac{k T n^{\circ}{ }_{i}}{\kappa}\left[\frac{8 a}{1-a^{2}} \ln \left(\frac{1+a}{1-a}\right)-\frac{8 a^{2}}{1-a^{2}}\right]
$$


Equation (3-91) can be put in the more convenient form,

$$
\Delta G(e l)=-\frac{8 a R T m^{\circ}}{1,000 \kappa\left(1-a^{2}\right)}\left[\ln \left(\frac{1+a}{1-a}\right)-a\right]
$$

Since one cannot obtain an analytical solution for the general case at finite plate distance, the charging process cannot be carried out. However, the necessary equations are available for $y_{0} \ll 1$.

We begin with the components of the system in an uncharged state and with the ion concentrations everywhere equal to their value at equilibrium. We again obtain

$$
\begin{aligned}
\rho^{\prime} & =\lambda \rho \\
& =\sum z_{i} e \lambda n_{i}
\end{aligned}
$$

Combining equation (3-93) with the expanded form of the Boltzmann equation and with equation (3-51) for the electric potential leads to

$$
\rho=-\frac{D}{4 \pi} \kappa^{2} \lambda \phi_{d} \cosh (\kappa d-\kappa x)
$$

Using equation (3-83) for charging in situ,

$$
\phi^{\prime}=\lambda \phi_{d} \cosh (\kappa d-\kappa x)
$$

We then find by straightforward integration that

Finally,

$$
\int_{0}^{d} \rho^{\prime} \phi^{\prime} d x=-\frac{D \kappa \lambda^{2} \phi^{2} d}{16 \pi}[\sinh (2 \kappa d)+2 \kappa d]
$$

$$
\Delta G(e l)=\int \frac{d \lambda}{\lambda} \int \rho^{\prime} \phi^{\prime} d x=-\frac{D \kappa \phi^{2} d}{32 \pi}[\sinh (2 \kappa d)+2 \kappa d]
$$

Using equation (3-52), equation (3-97) can be written as a function of $\sigma$ :

$$
\Delta G(e l)=-\frac{\pi \sigma^{2}}{D \kappa}\left[\frac{1}{\tanh \kappa d}+\frac{\kappa d}{\sinh ^{2} \kappa d}\right]
$$

\section{Osmotic free energy}

It is now necessary to find the osmotic free energy required to create the concentration gradients. Consider a given volume of any solution containing solute and a molality $m_{i}$. The free energy change for a change in the number of moles of solute, at constant water content, is

$$
d G=\bar{G}_{i} d n_{i}
$$

Since by definition $m_{i}$ is the number of moles of solvent per kilogram of water,

and

$$
n_{i}=m_{i} \frac{n_{\mathrm{H}_{2} \mathrm{O}}}{55.5}
$$

$$
d n_{i}=\frac{n_{\mathrm{H}_{2} \mathrm{O}}}{55.5} d m_{i}
$$

If the volume of the solution is one liter and the solution is dilute, $n_{\mathrm{H}_{2} \mathrm{O}}=55.5$, and

$$
d n_{i}=d m_{i} \quad \text { (one liter) }
$$


Substituting equation (3-102) in equation (3-99),

$$
d G(l)=\bar{G}_{i} d m_{i}
$$

where $G(l)$ is the free energy of one liter of solution. Therefore, if we transfer $d m_{i}$ moles from a solution at a concentration $m_{i}{ }^{\prime}$ to a solution at a concentration $m_{i}$, the free energy change is

$$
d G(l)=\bar{G}_{i} d m_{i}-\bar{G}_{i}{ }^{\prime} d m_{i}
$$

If the solutions are ideal,

$$
d G(l)=R T \ln \left(\frac{m_{i}}{m_{i}^{\prime}}\right) d m_{i}
$$

(In writing the double-layer theory we have already left the mutual ionic interactions out of account so that assuming ideal solutions here represents no loss in generality.) Therefore,

$$
\begin{gathered}
\Delta G(l)=R T \int_{m_{i^{\prime}}}^{m_{i}} \ln \left(\frac{m_{i}}{m_{i}{ }^{\prime}}\right) d m_{i} \\
=m_{i}{ }^{\prime} R T\left[1+\frac{m_{i}}{m_{i^{\prime}}{ }^{\prime}} \ln \frac{m_{i}}{m_{i^{\prime}}}-\frac{m_{i}}{m_{i^{\prime}}}\right]
\end{gathered}
$$

For convenience, let $U_{i}=m_{i} / m_{i}{ }^{\prime}$, so that

$$
\Delta G(l)=m_{i}{ }^{\prime} R T\left[1+U_{i} \ln U_{i}-U_{i}\right]
$$

For the diffuse layer at infinite plate distance, we wish to begin with an infinite volume at a concentration of $m^{\circ}{ }_{i}$ and find the free energy change to create the concentration gradient opposite one square centimeter of the surface. Since the free energy change per cubic centimeter is $\Delta G(l) / 1,000$, the free energy change for each volume element in the double layer is $[\Delta G(l) / 1,000] d x$, and we find the total free energy change per square centimeters of surface, $\Delta G(o s)$, by the integration

$$
\Delta G(o s)=\int_{0}^{\infty} \frac{\Delta G(l)}{1,000} d x
$$

Substituting equation (3-108), we obtain

$$
1,000 \Delta G(o s)=\int_{0}^{\infty} m^{\circ} R T\left[1+U_{i} \ln U_{i}-U_{i}\right] d x
$$

in which $U_{i}=m_{i} / m^{\circ}{ }_{i}$.

Beginning with the general case and $d=\infty$, we find from equation (3-19) that for cations

$$
U_{+}=\left(\frac{1-a e^{-\kappa x}}{1+a e^{-\kappa x}}\right)^{2}
$$

Letting $u=e^{\kappa x}$ and substituting equation (3-111) in equation (3-110), we obtain

$$
\begin{aligned}
1,000 \Delta G_{+}(o s)= & \int_{0}^{\infty} \frac{m^{\circ} R T}{\kappa u}\left[1+\left(\frac{u-a}{u+a}\right)^{2} \ln \left(\frac{u-a}{u+a}\right)^{2}\right. \\
& \left.-\left(\frac{u-a}{u+a}\right)^{2}\right] d u
\end{aligned}
$$


A similar equation may be found for $\Delta G_{-}(o s)$. After lengthy integration the result is

$$
\begin{gathered}
\Delta G(o s)=\Delta G_{+}+\Delta G_{-} \\
=\frac{8 a m^{\circ} R T}{1,000\left(1-a^{2}\right)_{\kappa}}\left[2 \ln \left(\frac{1+a}{1-a}\right)-3 a\right]
\end{gathered}
$$

The osmotic free energy can also be found for finite plate distance provided $y_{0} \ll 1$. In this case we must begin with each ion in an uncharged state and at a concentration equal to the final mean concentration given by equation (3-54). Thus, we put $U_{i}=m_{i} / \bar{m}_{i}$ to integrate equation (3-110). The result is

$$
\Delta G(o s)=\frac{2 \pi \sigma^{2}}{D \kappa}\left[\frac{1}{2 \tanh (\kappa d)}+\frac{\kappa d}{2 \sinh ^{2}(\kappa d)}-\frac{1}{\kappa d}\right]
$$

\section{Free energy of the plate}

It will be recalled that in the DH theory, the expression for the electric potential was divided into a term for the central ion and a term for the ion atmosphere. Only the term for the ion atmosphere was retained since we wished to calculate only the extra energy due to interactions. No analogous separation occurs in doublelayer theory, but in the present treatment of particles with a permanent charge, we wish to find only the extra free energy of the diffuse layer. This requires that the free energy of the plate be calculated and discarded. This situation is also different from the case treated by Verwey and Overbeek (1948) in which the charge is built up by the preferential adsorption of a "potential determining" ion.

The free energy of the plate is readily found. As the space charge is built up by charging the ions in situ, we also gradually build up the electrostatic charge on the plate. It follows that if $\sigma_{p}$ is the charge on the plate then

$$
\begin{gathered}
\sigma_{p}{ }^{\prime}=\lambda \sigma_{p} \\
\phi_{0}{ }^{\prime}=\lambda \phi_{0}
\end{gathered}
$$

where $\phi_{0}$ is the potential of the plate. Therefore,

$$
\begin{aligned}
\Delta G(p) & =\int \phi_{0}{ }^{\prime} d \sigma_{p}{ }^{\prime} \\
& =\int_{0}^{1} \phi_{0} \lambda \sigma_{p} d \lambda \\
& =\frac{\phi_{0} \sigma_{p}}{2}
\end{aligned}
$$

For the general case with $d=\infty$, substitution of equation (3-25) results in

$$
\Delta G(p)=\frac{2 e\left|z_{i}\right| n^{\circ} \phi_{0}}{\kappa} \sinh \left(\frac{y_{0}}{2}\right)
$$

This can be transformed to read

$$
\Delta G(p)=\frac{8 a R T m^{\circ}}{1,000 \kappa\left(1-a^{2}\right)} \ln \left(\frac{1+a}{1-a}\right)
$$

For the case of finite plate distance with $y_{0} \ll 1$, if we substitute equation (3-52) in equation (3-118), we find that

$$
\Delta G(p)=\frac{2 \pi \sigma^{2}}{D \kappa \cdot \tanh (\kappa d)}
$$




\section{Final free-energy equations}

The final equations for the free energy may now be found. For the case of infinite plate distance, the free energy per square centimeter, $\Delta G_{S}$, is

$$
\begin{aligned}
\Delta G_{S} & =\Delta G(e l)+\Delta G(o s)-\Delta G(p) \\
& =-\frac{8 a R T m^{\circ}}{1,000 \kappa\left(1-a^{2}\right)}\left[\ln \left(\frac{1+a}{1-a}\right)-a\right] \\
& +\frac{8 a R T m^{\circ}}{1,000 \kappa\left(1-a^{2}\right)}\left[2 \ln \left(\frac{1+a}{1-a}\right)-3 a\right] \\
& -\frac{8 a R T m^{\circ}}{1,000 \kappa\left(1-a^{2}\right)}\left[\ln \left(\frac{1+a}{1-a}\right)\right]
\end{aligned}
$$

Equation (3-123) simplifies to

$$
\Delta G_{S}=-\frac{16 a^{2} R T m^{\circ}}{1,000 \kappa\left(1-a^{2}\right)}
$$

For the case of finite plate distance with $y_{0} \ll 1$, if we substitute equations (3-97), (3-114) and (3-121) in equation (1-122), we obtain

$$
\begin{aligned}
\Delta G_{S}=-\frac{\pi \sigma^{2}}{D \kappa} & {\left[\frac{1}{\tanh (\kappa d)}+\frac{\kappa d}{\sinh ^{2}(\kappa d)}\right] } \\
+\frac{\pi \sigma^{2}}{D \kappa} & {\left[\frac{1}{2 \tanh (\kappa d)}+\frac{\kappa d}{2 \sinh ^{2}(\kappa d)}-\frac{1}{\kappa d}\right] } \\
& -\frac{\pi \sigma^{2}}{D \kappa \tanh (\kappa d)}
\end{aligned}
$$

Equation (3-125) simplifies to

$$
\Delta G_{S}=-\frac{2 \pi \sigma^{2}}{D \kappa}\left[\frac{1}{\tanh (\kappa d)}+\frac{1}{\kappa d}\right]
$$

\section{Content of the diffuse layer}

We must next find the appropriate composition variable against which to differentiate the equation for $\Delta G_{S}$. Returning to equation (3-32) for the mean concentration of ions between two points $x_{1}$ and $x_{2}$, we may let $x_{1}=0$, and find that

$$
\bar{m}_{+}=\frac{m_{+}^{\circ}}{x_{2}} \int_{0}^{x_{2}}\left(\frac{1-a e^{-\kappa x}}{1+a e^{-\kappa x}}\right) d x
$$

If we integrate equation (3-127), we obtain

$$
\begin{aligned}
\bar{m}_{+} & =\frac{m_{+}^{\circ}}{\kappa x_{2}}\left[\kappa x_{2}+\frac{4 a}{a+e^{\kappa x_{2}}}-\frac{4 a}{a+1}\right] \\
& =m_{+}^{\circ}+\frac{4 a m_{+}^{\circ}}{\kappa x_{2}\left(a+e^{\kappa x_{2}}\right)}-\frac{4 a m^{\circ}}{\kappa x_{2}(a+1)}
\end{aligned}
$$

The surface excess of ions between $x=0$ and $x=x_{2}$ is, therefore,

$$
\left(\bar{m}_{+}-m_{+}^{\circ}\right) x_{2}=\frac{4 a m_{+}^{\circ}}{\kappa\left(a+e^{\kappa x}{ }_{2}\right)}-\frac{4 a m_{+}^{\circ}}{\kappa(a+1)}
$$


Now, as $x_{2} \rightarrow \infty$, the right-hand side of equation (3-129) becomes

$$
\left(\bar{m}_{+}-m_{+}^{\circ}\right) x_{2}=-\frac{4 a m_{+}^{\circ}}{\kappa(1+a)}
$$

A similar derivation for the anions yields the equation

$$
\left(\bar{m}_{-}-m_{-}^{\circ}\right) x_{2}=\frac{4 a m_{-}^{\circ}}{\kappa(1-a)}
$$

Thus, although $x_{2}$ is infinite, we have previously shown that $\bar{m}_{+}=m^{\circ}+$ and that the difference $\bar{m}_{+}-m_{+}^{\circ}$ is zero. In this case, the product $\left(\bar{m}_{+}-m_{-}^{\circ}\right) x_{2}=0 \times \infty$ is finite. We obtain a similar result for anions. Therefore we define an "ionic content of the diffuse layer" $\left(\Delta_{i}\right)$ as the difference between the mean concentration and the concentration at infinity, multiplied by the complete thickness of the layer. Thus,

$$
\begin{aligned}
& \Delta_{+}=-\frac{4 a m^{\circ}}{1,000 \kappa(1+a)} \\
& \Delta_{-}=\frac{4 a m^{\circ}}{1,000 \kappa(1-a)}
\end{aligned}
$$

The subscript has been dropped from $m^{\circ}{ }_{i}$ since $m_{+}^{\circ}=m^{\circ}$ for symmetrical electrolytes. In defining the $\Delta_{i}$, equations (3-130) and (3-131) have been divided by 1,000 to give units of moles per $\mathrm{cm}^{2}$. Equations (3-132) and (3-133) give the excess or deficit of ions per $\mathrm{cm}^{2}$ of surface, and $\Delta G_{S}$ is the extra free energy due to these ions. Thus, the required derivative is $\partial \Delta G_{S} / \partial \Delta_{i}$.

For the case of finite plate distance and $y_{0} \ll 1$, we define the content of the diffuse layer as

$$
\Delta_{i}=\frac{\left(\bar{m}_{i}-m_{i}^{\circ}\right) d}{1,000}
$$

Following a derivation similar to the one just presented, we find

$$
\begin{aligned}
& \Delta_{+}=\frac{\sigma}{2\left|z_{i}\right| e} \\
& \Delta_{-}=-\frac{\sigma}{2\left|z_{i}\right| e}
\end{aligned}
$$

The form of these results might have been anticipated since the expansion of $\exp (-y)$ always leads to symmetrical concentration curves for cations and anions. The surface charge is therefore neutralized equally by an excess of one ion and a deficit of the other.

\section{Activity coefficients and filtrate composition}

The activity coefficients of the ions in the suspension are now readily found:

$$
\left(\frac{\partial \Delta G_{S}}{\partial \Delta_{i}}\right)=\mu_{i}-\mu^{*}{ }_{i}
$$

Since $\Delta G_{S}$ in equation (3-124) is in units of $R T$ per $\mathrm{cm}^{2}$ and $\Delta_{i}$ is in moles per $\mathrm{cm}^{2}$, $\mu^{*}{ }_{i}$ is in $R T$ per mole. Thus,

$$
\mu_{i}-\mu_{i}^{*}=R T \ln \gamma_{i}
$$


The differentiation of equation (3-124) is carried out as follows. We notice that the $\Delta_{i}$ is the excess or deficit of each ion species per $\mathrm{cm}^{2}$ of surface. It follows that

$$
\sum z_{i} e \Delta_{i}=\sigma
$$

(This equation can be verified by substitution of equations (3-132) and (3-133) and comparing the resulting equation with (3-25) after making appropriate changes in units.) Thus, to bring about the change in the ionic content of the diffuse layer required by equation (3-137), we make a displacement in $\sigma$. To do this it is convenient to define two new parameters, $B_{1}$ and $B_{2}$, as

$$
\begin{aligned}
& B^{2}{ }_{1}=\frac{8 \pi e^{2} N_{A} z^{2}{ }_{i}}{1,000 D k T} \\
& B_{2}=\frac{2 \pi e|z| \sigma}{D k T}
\end{aligned}
$$

If appropriate transformations in these equations are made, we obtain

$$
\begin{aligned}
B^{2}{ }_{1} & =\frac{\kappa^{2}}{m^{\circ}} \\
B_{2} & =-\frac{2 a \kappa}{1-a^{2}}
\end{aligned}
$$

Omitting the algebra, these equations lead to the following equation for $\Delta G_{S}$ as a function of $\Delta_{+}$:

Thus,

$$
\Delta G_{S}=\frac{8 R T B_{2}}{1,000 B^{2}{ }_{1}}-4 R T \Delta_{+}
$$

$$
\frac{d \Delta G_{S}}{d \Delta_{+}}=\frac{8 R T}{1,000 B^{2}} \frac{d B_{2}}{d \Delta_{+}}-4 R T
$$

Again omitting the algebra, we find

Thus,

$$
\Delta_{+}=\frac{2}{1,000 B^{2}{ }_{1}}\left[B_{2}-\kappa+\sqrt{\kappa^{2}+B^{2}{ }_{2}}\right]
$$

$$
\frac{d \Delta_{+}}{d B_{2}}=\frac{2}{1,000 B^{2}{ }_{1}}\left[1+\frac{B_{2}}{\sqrt{\kappa^{2}+B^{2}}}\right]
$$

Upon substituting equation (3-147) in equation (3-145), we find

Therefore,

$$
\frac{d \Delta G_{S}}{d \Delta_{+}}=-\frac{4 R T B_{2}}{B_{2}+\sqrt{\kappa^{2}+B^{2}{ }_{2}}}
$$

$$
\ln \gamma_{+}=-\frac{4 B_{2}}{B_{2}+\sqrt{\kappa^{2}+B^{2}{ }_{2}}}
$$

Rationalizing the denominator on the right, we obtain

$$
\ln \gamma_{+}=-\frac{4 B_{2}}{\kappa^{2}}\left(\sqrt{\kappa^{2}+B_{2}^{2}}-B_{2}\right)
$$


By a similar derivation we find that, for anions,

$$
\ln \gamma_{-}=\frac{4 B_{2}}{\kappa^{2}}\left(\sqrt{\kappa^{2}+B^{2}}+B_{2}\right)
$$

In fact, a second derivation for anions can be avoided simply by realizing that the behavior of an anion in a negatively charged system must be identical with the behavior of the cation in a positively charged system and by writing equation $(3-150)$ for this latter case. Since the sign of $B_{2}$ runs with the sign of $\sigma$, this merely involves changing the sign of $B_{2}$.

It is interesting to note that the activity coefficients in equations (3-150) and (3-151) depend only on the ratio $\kappa / B_{2}$. This ratio in turn is fixed by the ratio of $m^{\circ}$ to $\sigma$. In figure 14 the value of $\gamma_{+}$is plotted against $\kappa / B_{2}$. It is seen that as the

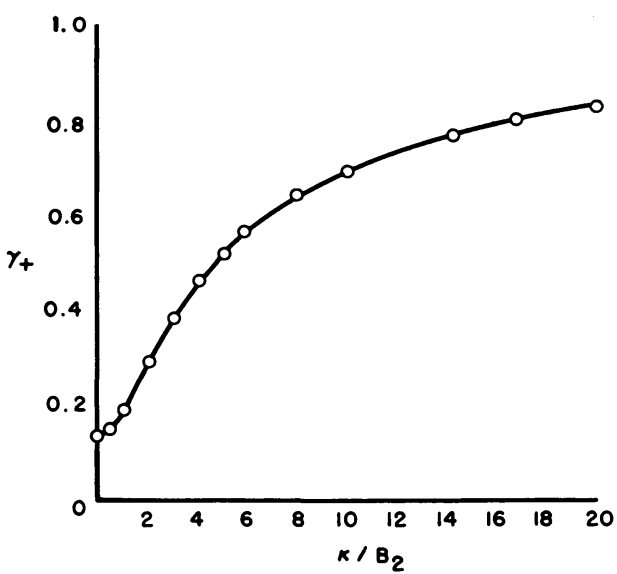

Figure 14. Cation activity coefficient in a system of negative particles as the relative salt content (measured by $\kappa / B_{2}$ ) increases, according to general theory. The plate distance is infinite.

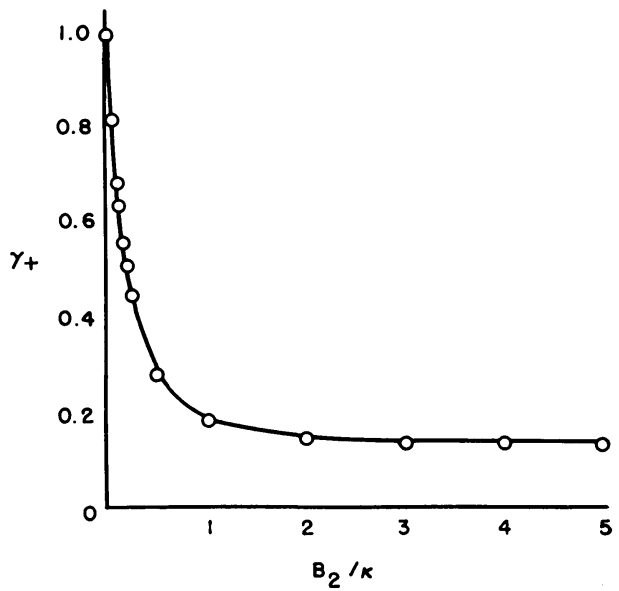

Figure 15. Cation activity coefficient in a system of negative particles as the salt content decreases from high values according to general theory. The plate distance is infinite.

relative salt content of the system increases, $\gamma_{+}$increases. This is of course quite the opposite of $\mathrm{DH}$ behavior. It results from the fact that as the relative salt content increases, the electric potential everywhere decreases according to equation (3-17). Thus the attraction energy between the cations and the negative particles decreases, and the activity coefficient increases.

In figure 15 the value of $\gamma_{+}$is plotted against the inverted ratio $B_{2} / \kappa$ for positive values of $\sigma$. This curve shows that $\gamma_{+}$rises to unity at infinite salt concentration, where all attraction energy between the cations and the plate has been "salted out." Actually, mutual ionic interactions would presumably prevent $\gamma_{+}$from rising to a value of unity.

In figure $16 \gamma_{-}$is plotted against $\kappa / B_{2}$ for positive values of $\sigma$. Here the activity coefficient decreases as the relative salt content increases, from large positive values down to unity. The limit of $\gamma_{-}$at high salt content is also shown in figure 17 where $\gamma_{-}$is plotted against the inverted ratio $B_{2} / \kappa$. The positive values of $\gamma_{-}$simply mean that, owing to the repulsion between the anions and the plate, the value of $\mu-\mu^{*}$ 
is positive. Thus, if the path were open, anions would spontaneously transfer from the suspension to the hypothetical ideal state.

The activity coefficients are not unity at infinite dilution. This result was anticipated in Section II where it was noted that in cases where we have inhomogeneity of charge distribution, the ions would not be expected to obey Henry's Law at infinite dilution. This, however, does not constitute any limitation on the Donnan equations of Section II. We have at hand the situation where the microscopic state of the suspension is determined by the double-layer theory and the macroscopic behavior may be found from the Donnan equations. Overbeek (1956) has also treated this system.

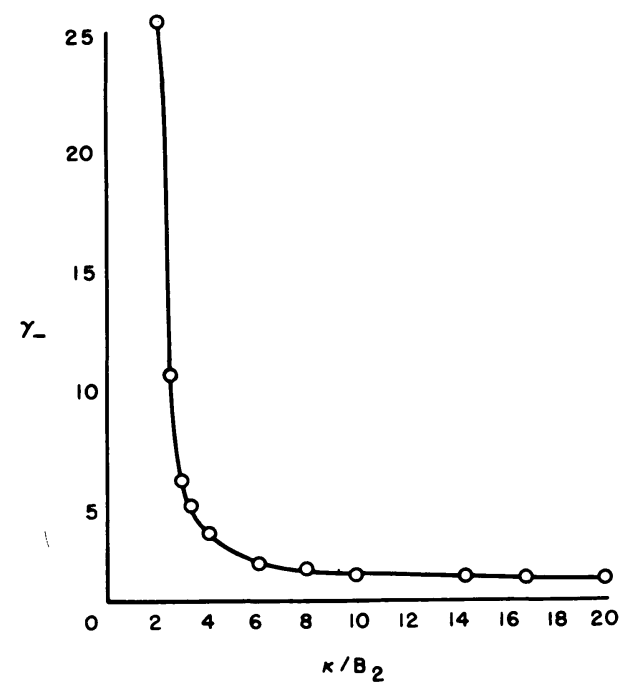

Figure 16. Anion activity coefficient in a system of negative particles as the relative salt content (measured by $\kappa / B_{2}$ ) increases, according to general theory. The plate distance is infinite.

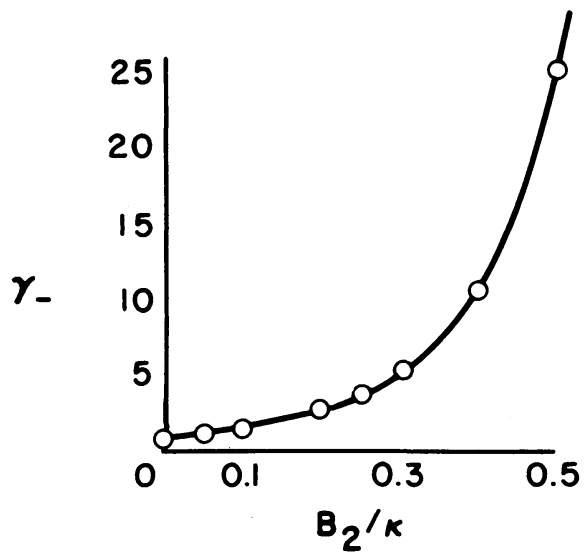

Figure 17. Anion activity coefficient in a system of negative particles as the salt content decreases from high values according to general theory. The plate distance is infinite.

The mean activity coefficient of salt in the system and the composition of filtrates in equilibrium with the suspension may now be found from the Donnan equations. For a symmetrical electrolyte,

$$
\gamma_{ \pm}=\left(\gamma_{+} \cdot \gamma_{-}\right)^{\frac{1}{2}}
$$

Using equations (3-151) and (3-152) we find

$$
\ln \gamma_{ \pm}=4\left(\frac{B_{2}}{\kappa}\right)^{2}
$$

To find the filtrate composition, it must be remembered that the condition $d=\infty$ requires an infinitely dilute suspension. Therefore, the mean molality of both ions in the suspension is $m^{\circ}$. Assuming a dilute filtrate at compsition $m_{f}$, equation (2-4) becomes

Therefore,

$$
\gamma^{2}{ }_{ \pm}\left(m^{\circ}\right)^{2}=m_{f}^{2}
$$

$$
\frac{m_{f}}{m^{\circ}}=\gamma_{ \pm}
$$


and from equation (3-153), we obtain

$$
\ln \frac{m_{f}}{m^{\circ}}=4\left(\frac{B_{2}}{\kappa}\right)^{2}
$$

In figure $18, m_{f} / m^{\circ}$ is plotted as a function of $\kappa / B_{2}$. The results show that at low relative salt content there is a large negative adsorption which rapidly decreases as the relative salt content increases. The curve is remarkably similar to figure 1 in Section II.

These results are in contradiction to the usual assumptions made in the doublelayer theory. It is frequently assumed that for $d=\infty$, the composition of the equilibrium filtrate must be equal to $m^{\circ}$. According to the present theory, the filtrate composition can be found only by calculating the chemical potential, and

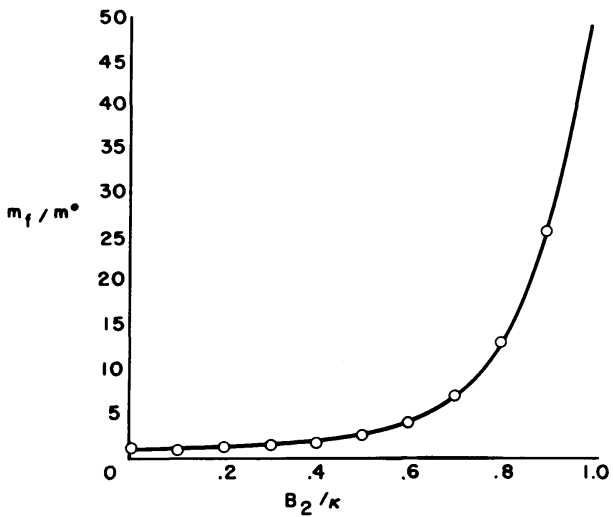

Figure 18. The ratio of the salt concentration in a filtrate to the salt concentration in an equilibrium suspension as the salt concentration decreases. The plate distance is infinite.

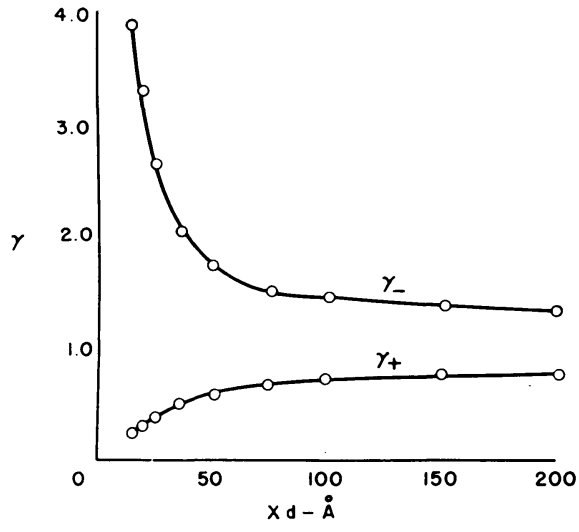

Figure 19. Cation and anion activity coefficients as a function of plate distance according to simplified theory. $\sigma=100 \mathrm{esu}$ per sq $\mathrm{cm}$ and $m^{o}=.00322 \mathrm{M}$.

thus the activity coefficient, of the ions in the suspension, and applying the Donnan equations. The results indicate that $\kappa / B_{2}$ must be in the order of 6 or 7 before $m_{f}$ is within a few per cent of $m^{\circ}$.

For the case of finite plate distance and $y_{0} \ll 1$, equation $(3-135)$ may be rewritten

$$
\Delta_{+}=\frac{2 B_{2}}{1,000 B^{2}}
$$

Using equations (3-126) and (3-157) and following a derivation parallel to the one given, the activity coefficients are found to be

$$
\begin{aligned}
& \ln \gamma_{+}=-\frac{4 B_{2}}{\kappa}\left[\frac{1}{\tanh \kappa d}+\frac{1}{\kappa d}\right] \\
& \ln \gamma_{-}=\frac{4 B_{2}}{\kappa}\left[\frac{1}{\tanh \kappa d}+\frac{1}{\kappa d}\right]
\end{aligned}
$$

Thus, the $\gamma_{i}$ are no longer determined by $\kappa / B_{2}$ since the plate distance enters the equations. In figure 19 the activity coefficients are plotted as a function of plate distance for $\sigma=100 \mathrm{esu} / \mathrm{cm}^{2}$ and $m^{\circ}=.00322$. Under these conditions, one cannot calculate with $d<13 \AA$ without violating the condition $y_{0} \ll 1$. 
In all cases for which $y_{0} \ll 1$, the symmetrical concentration curves also lead to symmetrical equations for the activity coefficient. Thus, the result of substituting equations (3-158) and (3-159) in equation (3-152) is

$$
\gamma_{ \pm}=1.0
$$

However, this result does not mean that negative adsorption is absent. The ionic activity coefficients have been defined such that

$$
a_{i}=\gamma_{i} \bar{m}_{i}
$$

Therefore, assuming a dilute filtrate, we find from equation (2-4) that

$$
\left(\bar{m}_{+} \cdot \bar{m}_{-}\right)=m_{f}^{2}
$$

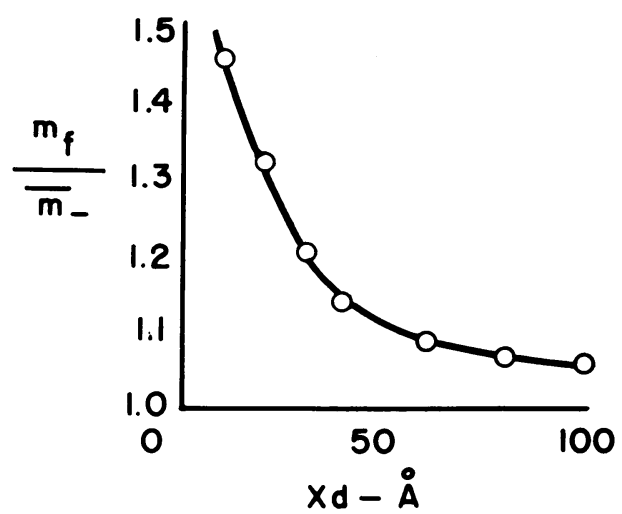

Figure 20. The ratio of the filtrate molality to the mean suspension molality as a function of plate distance according to simplified theory. $\sigma=100 \mathrm{esu}$ per sq $\mathrm{cm}$ and $m^{\circ}=.00322 \mathrm{M}$.

Substituting equation (3-54) for the mean concentrations and solving for $m_{f}$ the result is

$$
m_{f}=m^{\circ} \sqrt{1-\left(\frac{4 \pi \sigma z_{i} e}{\kappa^{2} k T D d}\right)^{2}}
$$

In figure 20 the ratio $m_{f} / m_{-}$is plotted as a function of the plate distance to show the negative adsorption. Equation (3-163) also shows that one cannot identify $m^{\circ}$ with the filtrate composition.

\section{Strong interactions}

A general analytical solution for the case of overlapping diffuse layers has not been found. However, the value of $y_{d}$ was shown to increase as the plate distance $d$ decreases. If the plate distance is sufficiently small that $y_{d} \gg 1$, then

$$
2 \cosh y=e^{y}+e^{-y} \cong e^{y}
$$

Equation (3-44) takes the form

$$
\frac{d y}{\sqrt{e^{y}-e^{y d}}}=-\kappa d x
$$


This equation can be integrated. The result is

$$
y=y_{d}-2 \ln \cos \left[\frac{\kappa(d-x) \exp \left(\frac{y_{d}}{2}\right)}{2}\right]
$$

The corresponding concentration equation is

$$
m_{i}=m^{d}{ }_{i} \cos \left[\frac{\kappa(d-x) \exp \left(\frac{y_{d}}{2}\right)}{2}\right]^{2 z_{i} /\left|z_{i}\right|}
$$

The use of these distribution equations to calculate chemical potentials would undoubtedly lead to more realistic results than the approximation $y_{0} \ll 1$. However, the use of equations (3-166) and (3-167) to find $\rho^{\prime}$ and $\phi^{\prime}$ for substitution into the charging equation leads to a lengthy expression which cannot be integrated.

\section{APPLICABILITY TO SOIL SYSTEMS}

It has been shown that the theory itself contains inherent limitations. While it may be reasonable in many systems to assume point charges, it does not seem reasonable to apply the theory to systems in which the theory itself predicts that the concentration gradients are confined to distances which are in the same order as the size of ions in solution. We have seen that this limits the theory to surface charge densities which are lower than that of clay systems. At best, then, one would expect the theory to predict the behavior of clay systems with low surface charge densities. It furthermore seems unlikely that any of the equations based on the condition $y_{0} \ll 1$ would apply to clays.

Other aspects of the theory have been examined in detail by Bolt (1955b) who concludes that the introduction of a number of effects such as mutual ionic interactions, dielectric saturation, and ionic polarization tend to have a cancelling effect. Bolt (1955a), Bolt and Peech (1953), and Bolt and Miller (1955) have also presented evidence to show the applicability of the Gouy theory to clay systems. The negative adsorption data mentioned at the end of Section II may also be interpreted as evidence for the Gouy theory. Marshall (1958) has reviewed further evidence. In recent years, then, there is a growing body of evidence that a diffuse layer does exist around soil particles. As previously explained, the presence of such a diffuse layer would also lead to the expectation of Donnan effects, in the sense that this term is used in Section II. However, the full evaluation of the significance of the diffuse layer must be postponed until the theory of ion-exchange in the next section has been presented. 


\section{Section IV \\ ION-EXCHANGE THEORY}

\section{ION-EXCHANGE EQUATIONS}

\section{Introduction}

In dealing with negatively charged colloids thus far, the main concern has been with the distribution of a single salt between two macroscopic phases. When we include more than one salt and focus attention on the cation distribution, the problem becomes one of cation exchange. Any equation which gives the distribution of cations between a suspension and its dialyzate may be called a cation-exchange equation.

Two categories of model systems have been employed for the derivation of cation-exchange equations. The first is the double-layer model, in which the Gouy distribution equations are used. In the second, the suspension is assumed to be composed of two discrete phases. One phase contains only the exchangeable ions and an infinitesimal amount of electrolyte, and the other is a homogeneous solution of electrolyte. This latter model is the basis for the mass-action approach and the statistical-thermodynamic approach. After examining the theory of these models, an attempt will be made to find which is most suited for soil systems.

\section{Double-layer theory}

A general analytical solution for the local distributions when $d \neq \infty$ cannot be found, since the equation for $d y / d x$ given by equation (3-44) leads to an elliptical integral. However, Ericksson (1952) has shown that to find the cation distribution, this equation does not need to be integrated. A cation-exchange equation can be found provided we deal with mixtures of symmetrical electrolytes.

Consider first a mixture of two uni-univalent electrolytes. The general form of the Poisson-Boltzmann equation given by equation (3-4) becomes

$$
\frac{d^{2} y}{d x^{2}}=\frac{8 \pi e^{2}}{D k T}\left[n_{1}^{\circ} \sinh y+n_{2}^{\circ} \sinh y\right]
$$

In the case of overlapping double layers, $n^{\circ}{ }_{i}$ is the concentration outside the plates. We define

Equation (4-1) becomes

$$
\lambda^{2}=\frac{8 \pi e^{2}}{D k T}
$$

$$
\frac{d^{2} y}{d x^{2}}=\lambda^{2}\left(n_{1}^{\circ}+n_{2}^{\circ}\right) \sinh y
$$

This integrates into

$$
\frac{d y}{d x}=\lambda \sqrt{2\left(n^{\circ}{ }_{i}+n \rho_{2}\right)\left(\cosh y-\cosh y_{d}\right)}
$$

Using equation (3-23) for the space charge, we obtain

$$
\sigma=\frac{D k T \lambda}{4 \pi \rho} \sqrt{2\left(n^{\circ}+n_{2}^{\circ}\right)\left(\cosh y_{0}-\cosh y_{d}\right)}
$$


We next find the fraction of this space charge which is due to the $i$ th electrolyte species:

Then,

$$
\sigma_{i}=\int_{0}^{d} \rho_{i} d x
$$

$$
\begin{aligned}
\rho_{i} & =\sum z_{i} e n_{i} \\
& =\sum z_{i} e n^{\circ}{ }_{i} \exp \left(-\frac{z_{i} e \phi}{k T}\right) \\
& =-\left(2 e n^{\circ}{ }_{i}\right) \sinh y
\end{aligned}
$$

Substituting equation (4-7) into equation (4-6), we find that

$$
\sigma_{i}=-\int_{0}^{d}\left(2 e n_{i}^{\circ}\right) \sinh y d x
$$

Solving equation (4-4) for $d x$ and substituting the result in equation (4-8), the resulting expression can be integrated.

Letting

the result is

$$
v=\cosh y
$$

$$
\sigma_{i}=\frac{4 e n_{1}^{\circ}}{\lambda} \sqrt{\frac{v_{0}-v_{d}}{2\left(n^{\circ}{ }_{1}+n_{2}^{\circ}\right)}}
$$

Then, dividing equation (4-10) by equation (4-5), we obtain

$$
\frac{\sigma_{i}}{\sigma}=\frac{n_{1}^{\circ}}{n_{1}^{\circ}+n_{2}^{\circ}}
$$

Thus, the fraction of the surface neutralized by either electrolyte is simply equal to its fraction outside the plates. This result might have been anticipated since the Gouy distribution equations contain no ionic specificities.

Consider next an unsymmetrical exchange between two symmetrical electrolytes. Let electrolyte species 1 be a uni-univalent salt and species 2 be a di-divalent salt. The Poisson-Boltzmann equation becomes

$$
\frac{d^{2} y}{d x^{2}}=\lambda^{2}\left[n^{\circ}{ }_{i} \sinh y+2 n^{\circ} \sinh 2 y\right]
$$

Upon integrating once and using equation (3-23) for the space charge, we find that

$$
\sigma=\frac{D k T}{4 \pi e} \sqrt{2 n^{\circ}{ }_{1}\left(v_{0}-v_{d}\right)+4 n_{2}^{\circ}\left(v^{2}{ }_{0}-v_{d}^{2}\right)}
$$

Equation (4-6) may again be used to find the fraction of the surface neutralized by either electrolyte. Thus we find that

$$
\sigma_{1}=-\frac{e n_{1}^{\circ}}{\lambda \sqrt{n^{\circ}{ }_{2}}} \ln \left[\frac{\lambda \sigma \sqrt{n^{\circ}}+4 e v_{0} n^{\circ}{ }_{2}+e n_{1}^{\circ}}{\left(4 v_{d} n_{2}^{\circ}+n^{\circ}\right) e}\right]
$$

Equation (4-13) may be solved for $v_{0}$ and used to eliminate $v_{0}$ from equation (4-14). The result is

$$
\sigma_{1}=\frac{e n_{1}^{\circ}}{\lambda \sqrt{n^{\circ}}} \ln \left[\frac{\lambda \sigma \sqrt{n^{\circ}}{ }_{2}+\sqrt{e^{2}\left(n_{1}^{\circ}+4 n_{2} v_{d}\right)^{2}+n_{2}^{\circ} \lambda^{2} \sigma^{2}}}{\left(4 v_{d} n^{\circ}{ }_{2}+n^{\circ}{ }_{1}\right) e}\right]
$$


Equation (4-15) can be transformed to read

and

$$
\sigma_{1}=\frac{e n_{1}^{\circ}}{\lambda \sqrt{n^{\circ}}} \sinh ^{-1}\left[\frac{\lambda \sigma \sqrt{n^{\circ}}}{n_{1}^{\circ}+4 v_{d} \sqrt{\left(n_{2}^{\circ}\right) e}}\right]
$$

$$
\frac{\sigma_{1}}{\sigma}=\frac{e n^{\circ}{ }_{1}}{\lambda \sigma \sqrt{n^{\circ}}} \sinh \mathrm{h}^{-1}\left[\frac{\lambda \sigma \sqrt{n^{\circ}}}{n_{1}^{\circ}+4 v_{d} \sqrt{\left(n^{\circ}{ }_{2}\right)} e}\right]
$$

Equation (4-17) can be put in the form written by Bolt (1955a):

in which

$$
\frac{\Gamma_{1}}{\Gamma}=\frac{r}{\Gamma \cdot \sqrt{\beta}} \sinh ^{-1}\left[\frac{\Gamma \cdot \sqrt{\beta}}{r+4 v_{d} \sqrt{m^{\circ}{ }_{2}}}\right]
$$

$$
r=\frac{m_{1}^{\circ}}{\sqrt{m^{\circ}{ }_{2}}}
$$

is called the "reduced ratio." $\Gamma$ is the surface charge density in me/cm ${ }^{2}$ and $\beta$ is a constant given as

$$
\beta=\frac{8,000 \pi \mathcal{F}^{2}}{D R T}
$$

where $\mathcal{F}=2.892 \times 10^{11} \mathrm{esu} / \mathrm{me}$. At $25^{\circ} \mathrm{C}, \beta=1.080 \times 10^{15}$. Equation (4-18) gives the fraction of the surface charge neutralized by the monovalent electrolyte and may therefore be considered an ion-exchange equation, provided the $m^{\circ}{ }_{i}$ (the concentration outside the plates) can be identified with the filtrate. In Section III it was argued that this may not be the case, but we shall find in the next section that this argument has no effect on the ion-exchange equation. In most applications of equation (4-18), the value of $v_{d}$ is taken as unity. This amounts to cosh $y_{d}=1.0$ or $y_{d}=0$, that is, infinite plate distance.

\section{Mass-action theory}

In order to apply the mass-action theory, a model must be assumed in which a suspension consists of two discrete phases. We may then speak unambiguously of the "exchanger phase" and the "solution phase" in the suspension. It is not necessary to assume any further details. The process of cation exchange may be represented as an interchange of ions between the exchanger phase and the solution phase as follows:

$$
\nu_{A} A(a d)+\nu_{B} B \rightarrow \nu_{A} A+\nu_{B} B(a d)
$$

The suffix $(a d)$ represents an adsorbed ion and the absence of a suffix denotes an ion in solution. The free energy change for this process is

$$
\Delta G=\nu_{B} \bar{G}_{B(a d)}+\nu_{A} \bar{G}_{A}-\nu_{B} \bar{G}_{B}-\nu_{A} \bar{G}_{A(a d)}
$$

The partial molal free energies may be separated into chemical potentials and the electric potential of each phase according to the equation

$$
\bar{G}_{i}=\mu_{i}+z_{i} F_{y} \psi
$$


Equation (4-21) becomes

$$
\begin{aligned}
\Delta G=\nu_{B}\left(\mu_{B(a d)}+z_{B} F_{y} \psi^{E}\right) & +\nu_{A}\left(\mu_{A}+z_{A} F_{y} \psi^{S}\right) \\
& -\nu_{B}\left(\mu_{B}+z_{B} F_{y} \psi^{S}\right)-\nu_{A}\left(\mu_{A(a d)}+z_{A} F_{y} \psi^{E}\right)
\end{aligned}
$$

Superscript $E$ refers to the exchanger phase and $S$ to the solution. However, electroneutrality requires that

$$
\nu_{B} z_{B}=\nu_{A} z_{A}
$$

The electric potentials therefore cancel out of equation (4-22), so that

$$
\Delta G=\nu_{B} \mu_{B(a d)}+\nu_{A} \mu_{A}-\nu_{B} \mu_{B}-\nu_{A} \mu_{A(a d)}
$$

The possibility also exists of an electric potential difference between the solution in the suspension and an equilibrium filtrate. Any such potential difference also cancels out of the free energy equation, and we may therefore regard equation (4-24) as the $\Delta G$ for an exchange between the exchanger phase and a filtrate. It is this fact which also allows the identification of the $m^{\circ}{ }_{i}$ with the molalities in the filtrate in the case of the double-layer equation of the preceding section. For an exchange in which all ions are in their standard states, equation (4-24) becomes

$$
\Delta G^{\circ}=\nu_{B} \mu_{B(a d)}^{\circ}+\nu_{A} \mu_{A}^{\circ}-\nu_{B} \mu_{B}^{\circ}-\nu_{A} \mu_{A(a d)}^{\circ}
$$

Subtracting equation (4-25) from equation (4-24) and using the definition of ionic activity according to the equation $\mu_{i}-\mu_{i}^{\circ}=R T \ln a_{i}$, we have

$$
\Delta G-\Delta G^{\circ}=R T \ln \left(\frac{a^{\nu_{B}}(a d) \cdot a^{\nu_{A}}}{a^{\nu_{A} A(a d)} \cdot a^{\nu_{B}}}\right)
$$

At equilibrium, $\Delta G=0$, so that the equilibrium condition is

Therefore, at equilibrium,

$$
\Delta G^{\circ}=-R T \ln \left(\frac{a^{\nu_{B}}(a d) \cdot a^{\nu_{A}}}{a^{\nu_{B}(a d)} \cdot a^{\nu_{B}}}\right)
$$

$$
\frac{a^{\nu_{B}}(a d) \cdot a^{\nu_{A}}}{a^{\nu_{A}} A(a d) \cdot a^{\nu_{B}}}=\exp \left(-\frac{\Delta G^{\circ}}{R T}\right)=k
$$

Here, $k$ is the equilibrium constant. Equation (4-28) may be regarded as a massaction equation.

The derivation of equation (4-28) is exactly parallel to the treatment of a chemical reaction. There is, however, nothing in the derivation which assumes that a molecular species is formed by the ion on the surface. The mass action approach has been criticized on the ground that it implies an exchanger phase of homogeneous composition. This condition is not required for the derivation of equation (4-28). $\mu_{i}$ must be uniform in the exchanger phase at equilibrium, but the composition need not be uniform.

The standard state for ions in the exchanger phase is sometimes taken as the same as that selected for ions in solution (Babcock, Davis, and Overstreet, 1951). This results in $\Delta G^{\circ}=0$, since the exchange reaction will involve the transfer of ions between two states having the same free energy. Then, $k=1$ and

$$
\frac{a^{\nu_{B}} B_{(a d)} \cdot a^{\nu_{A}}}{a^{\nu_{A} A(a d)} \cdot a^{\nu_{B}}}=1
$$


With this selection of standard state, all thermodynamic equilibrium constants become equal to unity. This procedure is perfectly acceptable for certain purposes, but it is uninformative from the point of view of predicting the cation distribution.

The activity ratio $a^{\nu_{A}} A / a^{n_{B}}$ in the solution phase is thermodynamically well defined. It is somewhat clumsy to write the general case, but if each electrolyte has a common anion $X$ of any valency, it can be shown that

$$
\frac{a^{\nu_{A}}}{a^{\nu_{B}}}=\frac{\left(\gamma^{\left(\nu \nu_{A} / \nu_{+}\right)}\right)_{A} m^{\nu_{A}}}{\left(\gamma^{\left(\nu \nu_{B} / \nu_{+}\right)}\right)_{B} m^{\nu_{B}}}
$$

where $\nu$ is the number of ions dissociated from either salt $A_{\nu_{+}} X_{\nu_{-}}$or $B_{\nu_{+}} X_{\nu_{-}}$.

To obtain a cation-exchange equation, a theory for the activity of the ions in the exchanger phase must be found. One of the first theories was advanced by Vanselow (1932) who assumed that the exchanger phase could be treated on analogy with an ideal solid solution. On this assumption, the activities of adsorbed ions are set equal to their mole fraction in the exchanger phase. Equation (4-28) becomes

$$
\frac{N^{\nu_{B} B(a d)} \cdot a^{\nu_{A}}}{N^{\nu_{A} A(a d)} \cdot a^{\nu_{B}}}=k_{v}
$$

where $N$ is the mole fraction.

Vanselow found that many systems obey (4-31). When the value of the function on the left does not yield a constant, $k_{v}$ may be called a "selectivity function."

Argersinger, Davidson, and Bonner (1950) have extended Vanselow's theory for the case where the selectivity function $k_{v}$ is not a constant. Activity coefficients for adsorbed ions are defined as

Equation (4-28) becomes

$$
f_{i}=\frac{a_{i(a d)}}{N_{i(a d)}}
$$

$$
\frac{f^{\nu_{B}} \cdot N^{\nu_{B}}(a d) \cdot a^{\nu_{A}}}{f^{\nu_{A}} \cdot N^{\nu_{A}}{ }^{\nu_{A}}(a d) \cdot a^{\nu_{B}}}=\frac{f^{\nu_{B}}}{f^{\nu_{A}}} k_{v}=k
$$

The activity coefficients are evaluated by applying the Gibbs-Duhem equation to the exchanger phase. We obtain

$$
\begin{gathered}
N_{A} d \mu_{A}+N_{B} d \mu_{B}=0 \\
N_{A} d \ln f_{A}+d N_{A}+N_{B} d \ln f_{B}+d N_{B}=0
\end{gathered}
$$

Since $N_{A}+N_{B}=1.0$,

$$
N_{A} d \ln f_{A}+N_{B} d \ln f_{B}=0
$$

From equation (4-33), we find that

$$
\nu_{B} \ln f_{B}-\nu_{A} \ln f_{A}+\ln k_{v}=\ln k
$$

Differentiating equation (4-37), we obtain

$$
\nu_{B} d \ln f_{B}=\nu_{A} d \ln f_{A}-d \ln k_{v}
$$

Substituting equation (4-38) in equation (4-36) for $d \ln f_{B}$, and solving for $d \ln f_{A}$, we obtain

$$
d \ln f_{A}=\frac{N_{B}}{\nu_{B} N_{A}+\nu_{A} N_{B}} d \ln k_{v}
$$


It is convenient to define a parameter $x$ as

Equation (4-39) becomes

$$
x=\frac{\nu_{A} N_{B}}{\nu_{B} N_{A}+\nu_{A} N_{B}}
$$

$$
\nu_{A} d \ln f_{A}=x d \ln k_{v}
$$

Equation (4-41) is more convenient in the form

$$
\nu_{A} d \ln f_{A}=d\left(x \ln k_{v}\right)-\ln k_{v} d x
$$

If we select $N_{A}=1$ as the standard state for the $A$ ions, then according to equation (4-32), $f_{A}=1$ at $N_{A}=1$, since the activity must be unity in the standard state. Then,

$$
\nu_{A} \ln f_{A}=x \ln k_{v}-\int_{0}^{x} \ln k_{v} d x
$$

since $x=0$ at $N_{A}=1$. By an entirely parallel derivation we find the activity coefficient of $B$ based on a standard state of $N_{B}=1$ :

$$
\nu_{B} \ln f_{B}=(x-1) \ln k_{v} \int_{1}^{x} \ln k_{v} d x
$$

Therefore, when $k_{v}$ is variable, the activity coefficients for $A$ and $B$ can be found by measuring $k_{v}$ as a function of $x$ and using equations (4-43) and (4-44). When these equations are substituted in equation (4-37) we find the equilibrium constant:

$$
\ln k=\int_{0}^{1} \ln k_{v} d x
$$

Argersinger and his associates have evaluated a number of such thermodynamic constants for synthetic exchangers.

Equations (4-43) and (4-44) are expressions for single ionic activity coefficients in the exchanger phase. Thus, although the derivations appear straightforward, an extra-thermodynamic assumption must have entered. This assumption is that the activity of an adsorbed ion is uniquely determined by its mole fraction in the exchanger phase. Thus it is assumed that the standard chemical potential is completely specified by assigning $N_{i}=1$ as standard state. In fact, this may not be the case. At unit mole fraction the chemical potential of an adsorbed ion may be a function of the single electrolyte concentration with which the exchanger phase is in equilibrium. In such a case, the values of $f_{A}, f_{B}$ and $k$ will be found to depend upon the particular path of composition variables over which the parameter $x$ is varied during the integration of equations (4-43), (4-44) and (4-45). A better insight into the nature of this problem will be found in the course of a statisticalthermodynamic analysis.

An interesting standard state for adsorbed ions was presented by Gaines and Thomas (1953). For adsorbed ions, they select as standard state a condition in which the ions are at unit mole fraction and in equilibrium with a solution of the ions at infinite dilution. A practical difficulty with this selection is that weakly adsorbed cations hydrolyze as the salt concentration in the solution decreases. Thus, extrapolation of the data to the standard state may be impossible. It might be imagined that this difficulty could be overcome by specifying an arbitrary solution concentration with which the adsorbed ions at unit mole fraction are in 
equilibrium. This procedure has a curious result. Suppose the arbitrary concentration of solution ions chosen is $m_{i}{ }^{\prime}$. This may be assumed to fix the chemical potential of the solution ions at some value, $\mu_{i}{ }^{\prime} . \Delta G^{\circ}$ is now found by summing the $\Delta G$ values of the following processes:

1. $\nu_{A}$ moles of $A$ ions are transferred from an infinite surface at unit mole fraction of $A$ to a solution at composition $m_{A}{ }^{\prime}$ with which it is at equilibrium:

$$
\nu_{A} A_{a d}\left(N_{A}=1\right) \rightarrow \nu_{A} A\left(m_{A}{ }^{\prime}\right)
$$

2. $\nu_{A}$ moles of $A$ ions are transferred from the solution $m_{A}{ }^{\prime}$ of the ions to its standard state $\left(\mu_{A}=\mu_{A}^{\circ}\right)$ :

$$
\nu_{A} A\left(m_{A}{ }^{\prime}\right) \rightarrow \nu_{A} A\left(\mu_{A}^{\circ}\right)
$$

3. $\nu_{B}$ moles of $B$ are transferred from a solution in its standard state to a solution at concentration $m_{B}{ }^{\prime}$ :

$$
\nu_{B} B\left(\mu_{B}^{\circ}\right) \rightarrow \nu_{B} B\left(m_{B}{ }^{\prime}\right)
$$

4. $\nu_{B}$ moles of $B$ are transferred from the solution $m_{B}{ }^{\prime}$ to an infinite surface at unit mole fraction of $B$ with which it is in equilibrium:

$$
\nu_{B} B\left(m_{B}{ }^{\prime}\right) \rightarrow \nu_{B} B_{a d}\left(N_{B}=1\right)
$$

The $\Delta G$ for the net process is $\Delta G^{\circ}$. For processes 1 and 4 , we obtain $\Delta G=0$, since they occur in equilibrium. Summing the $\Delta G$ values of processes (2) and (3), we obtain

$$
\Delta G^{\circ}=\nu_{A} \bar{G}_{A}^{\circ}-\nu_{A} \bar{G}_{A}^{\prime}+\nu_{B} \bar{G}_{B}{ }^{\prime}-\nu_{B} \bar{G}^{\circ}{ }_{B}
$$

Here, each value of $\bar{G}$ refers to the solution, so that (as explained in Section I)

$$
\begin{gathered}
\Delta G^{\circ}=\nu_{A} \mu_{A}^{\circ}-\nu_{A} \mu_{A}{ }^{\prime}+\nu_{B} \mu_{B}{ }^{\prime}-\nu_{B} \mu^{\circ}{ }_{B} \\
=R T \ln \frac{\left(a_{B}{ }^{\prime} \nu_{B}\right.}{\left(a_{A}{ }^{\prime}\right)^{\nu_{A}}}
\end{gathered}
$$

Thus, whenever we choose a standard state for adsorbed ions by selecting an arbitrary mole fraction (unity or otherwise) and an arbitrary solution concentration with which the surface is in equilibrium, $\Delta G^{\circ}$ simply becomes a function of the ratio of the ionic activities at the arbitrarily chosen concentrations.

This is scarcely the desired result. One may arbitrarily select standard states, but they should be selected in such a way that $\Delta G^{\circ}$ measures the relative affinity of the ions for the surface when all components are in their standard states. To accomplish this, the components must not be in equilibrium when they are in their standard states or in reference states.

The problem, then, becomes one of selecting states for adsorbed ions and solution ions which are not in equilibrium with one another so that $\Delta G^{\circ}$ measures the tendency of ions to either enter or leave the exchanger phase. Doubtless many such states may be chosen. There is some convenience in selecting standard states such that the ions have the same mole fraction on one and the same surface. This can only occur at $N_{i}=0.5$. Furthermore, since the activity is always unity in the standard state, we know for this selection that $f_{i}^{\circ}=2$ for all ions. While this may seem unusual, it is a convenient method of procedure. (The activity coefficients for ions in solution are not equal to unity in the standard state. They are equal to unity 
at infinite dilution rather than when $a_{i}=1$.) The value of $k$ and $\Delta G^{\circ}$ may now be found by the method given above, provided that the mole fraction is sufficient to specify the chemical potential. $\Delta G^{\circ}$ then represents the relative affinity of the ions for the surface when the adsorbed ions are each at the same mole fraction and the solution ions are in their standard states. For the special case in which the value of the selectivity function $k_{v}$ is found to be a constant experimentally, we conclude from equation $(4-33)$ that the ratio $f_{B}{ }^{\nu_{B}} / f_{A}{ }^{\nu_{A}}$ must be a constant. Since $f^{\circ}{ }_{B}=$ $f_{A}^{\circ}=2$, we find that

$$
k=k_{v}(2)^{v_{B}-v_{A}}
$$

The value of $\Delta G^{\circ}$ is thus

$$
\begin{aligned}
\Delta G^{\circ} & =-R T \ln k_{v}-\left(\nu_{B}-\nu_{A}\right) R T \ln 2 \\
& =-R T \ln k_{v}-\left(\nu_{B}-\nu_{A}\right)(0.693) R T
\end{aligned}
$$

Since $\Delta G^{\circ}$ refers to the standard states discussed above, a more rational measure of relative affinities for the case of unsymmetrical exchanges is provided. This also illustrates the advantage of having both adsorbed ion species in their standard states on one and the same surface.

\section{Osmotic effects}

In deriving equations for the distribution of cations from either the double-layer theory or the mass-action model, nothing has been said about the equilibrium conditions for water.

The equations for the chemical potential of a single ion have already been found in Section II. According to equation $(2-56)$, we know that for an ion in the suspension

$$
\mu_{i}^{\mathrm{I}}-\mu_{i}^{\circ}=R T \ln a_{i}^{p}+\bar{V}_{i}\left(P_{\mathrm{eq}}^{\mathrm{I}}-P^{\circ}\right)
$$

in which $a^{p}$ is the activity of the ion at standard pressure $P^{\circ}$, and $P_{\mathrm{eq}}^{\mathrm{I}}$ is the pressure which must be applied to the suspension to equilibrate the water in the suspension with the solution phase which is at $P^{\circ}$. Subtracting equation (4-25) from equation (4-24) and substituting equation (2-56), we obtain

$$
\begin{aligned}
\Delta G-\Delta G^{\circ}=\nu_{B} & \left.R T \ln a_{B(a d)}^{p}+\bar{V}_{B} \mathrm{I}\left(P_{\text {eq }}-P^{\circ}\right)\right]+R T \ln a^{\nu_{A}} \\
& -\nu_{A}\left[R T \ln a_{A(a d)}^{p}+\bar{V}_{A}{ }^{\mathrm{I}}\left(P_{\mathrm{eq}}-P^{\circ}\right)\right]-R T \ln a^{\nu_{B}}
\end{aligned}
$$

At equilibrium, $\Delta G=0$ and

$$
R T \ln \frac{\left[a^{p}{ }_{B(a d)}\right]^{\nu_{B}}\left(a_{A}\right)^{\nu_{A}}}{\left[a_{A(a d)}^{p}\right]^{\nu_{A}}\left(a_{B}\right)^{\nu_{B}}}+\left(\nu_{B} \bar{V}_{B}^{\mathrm{I}}-\nu_{A} \bar{V}_{A}^{\mathrm{I}}\right)\left(P_{\mathrm{eq}}^{\mathrm{I}}-P^{\circ}\right)=-\Delta G^{\circ}
$$

The thermodynamic equilibrium constant is still given by $\Delta G^{\circ}=-R T \ln k$. Therefore,

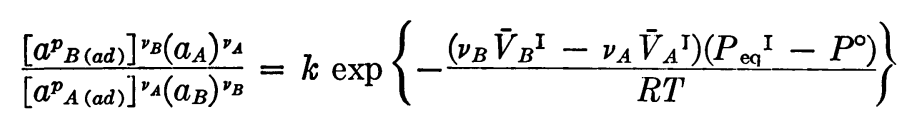


If, at standard pressure, the activity of adsorbed ions is determined by the mole fraction, then

$$
k_{v}=k\left(\frac{f^{\nu_{A}}}{f^{\nu_{B}}}\right) \exp \left\{-\frac{\left(\nu_{B} \bar{V}_{B}^{\mathrm{I}}-\nu_{A} \bar{V}_{A}^{\mathrm{I}}\right)\left(P_{\mathrm{eq}}^{\mathrm{I}}-P^{\circ}\right)}{R T}\right\}
$$

Thus, in swelling systems, $k_{v}$ will not in general be constant even if $f^{\nu_{A}}{ }_{A} / f^{\nu_{B}}$ is constant, since $P_{\text {eq }}^{\mathrm{I}}-P^{\circ}$ will vary with composition.

\section{Anion effects}

It is of some interest to note that the ratio of solution ion activities, which is required by mass-law equations, depends upon the nature of the anion present. This is seen in equation (4-30). For example, if we have $\mathrm{Na}^{+}$and $\mathrm{Ca}^{++}$present as chlorides, equation (4-30) gives,

$$
\frac{a^{2} \mathrm{Na}^{+}}{a_{\mathrm{Ca}^{++}}}=\frac{\gamma_{ \pm\left(\mathrm{NaCl}^{+}\right)}^{4} \cdot m^{2} \mathrm{Na}^{+}}{\gamma_{ \pm\left(\mathrm{CaCl}_{2}\right)}^{3} \cdot m_{\mathrm{Ca}^{++}}}
$$

However, if the $\mathrm{Na}^{+}$and $\mathrm{Ca}^{++}$are present as sulfates,

$$
\frac{a^{2} \mathrm{Na}}{a_{\mathrm{Ca}^{++}}}=\frac{\gamma^{3} \pm\left(\mathrm{Na}_{2} \mathrm{SO}_{4}\right) \cdot m^{2} \mathrm{Na}}{\gamma_{ \pm\left(\mathrm{CaSO}_{4}\right)}^{2} \cdot m_{\mathrm{Ca}^{++}}}
$$

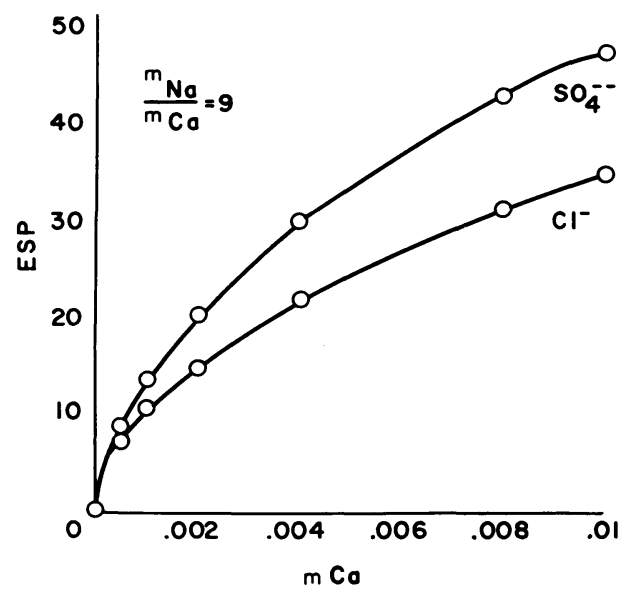

Figure 21. Exchangeable sodium percentage as a function of calcium concentration at a fixed cation ratio for different anion species. The curves were calculated from Vanselow's equation.

Thus, at identical values of $m^{2} \mathrm{Na} / m_{\mathrm{Ca}^{++}}$, the activity ratios are different. As an example of such effects, the equilibrium exchangeable sodium percentage has been calculated as a function of $m_{\mathrm{Ca}^{++}}$for a ratio of $m_{\mathrm{Na}^{+}} / m_{\mathrm{Ca}^{++}}=9$. Vanselow's equation was used for the calculations, and $\gamma_{ \pm}$values were estimated from available data with the aid of the principle of ionic strength. The results, shown in figure 21, indicate that the anion effect can be appreciable.

\section{Statistical thermodynamics}

In the double-layer theory, the charge density on the surface of the particles is assumed to be uniform and the ions are taken as point charges. However, the actual charge on colloidal particles may be due to the dissociation of a surface group, the preferential adsorption of a given ion, or isomorphic substitution of ions within 
the colloid. Thus, the charges arise at specific points on or near the surface, and at any given time an adsorbed ion may be associated with a particular adsorption site. In the language of statistical thermodynamics, such adsorbed ions are said to be localized, whereas in double-layer theory the adsorbed ions are nonlocalized.

An ion-exchange equation, derived for localized adsorbed ions by the methods of Fowler and Guggenheim (1939), was first presented by Krishnamoorthy, Davis, and Overstreet (1949) and developed by Davis $(1950 a, b)$ and Davis and Rible (1950). The model to which the statistical thermodynamics is applied may be stated as follows:

(a) The surface is composed of identical, discrete sites with unit electric charge.

(b) The total number of ionic charges on the surface is equal to the number of oppositely charged sites, and each ion is localized.

(c) The interaction energy between a site and its adsorbed ion is not affected by neighboring ions.

(d) The mutual interaction energy of the adsorbed ions is the same for ions of the same valence.

We may note in passing that condition (b) involves the exclusion of anions, and the model thus consists of two discrete phases. Although each ion is assumed to be localized at a given time, its position is not rigidly fixed and the adsorbed ions may constitute a more or less diffuse layer.

When statistical thermodynamics is applied to this model for symmetrical ion exchanges, the result is identical with Vanselow's equation (4-31). In a model which meets conditions (a) to (d), the chemical potential is specified by the mole fraction because the energy is independent of configuration, that is, the way in which the ions are distributed among the sites. Furthermore the constancy of the selectivity function results from the constancy of the specific ionic interactions.

For unsymmetrical exchanges, two additional characteristics must be assigned to the model:

(a) A polyvalent ion of valency $z_{i}$ must occupy a number of nearest neighbor sites of $z_{i}$.

(b) When a polyvalent ion of valency $z_{i}$ replaces $z_{i}$ monovalent ions, the mutual interaction energy is decreased by the interaction energy of the monovalent ions. The resulting ion-exchange equation reads

$$
\frac{\left(a_{A}\right)^{\nu_{A}}\left(n_{B}\right)^{\nu_{B}}}{\left(a_{B}\right)^{\nu_{B}}\left(n_{A}\right)^{\nu_{A}} \cdot\left[\Sigma q_{i} n_{i}\right]^{\nu_{B-\nu_{A}}}}=k_{D}
$$

where $n_{i}$ is the number of moles of adsorbed ions. $q_{i}$ is a parameter which depends upon the valence of the ion and the geometry of the surface as follows:

$$
q_{i}=z_{i}-\frac{2 z_{i}}{Y}+\frac{2}{Y}
$$

where $z_{i}$ is valence and $Y$ is the number of adsorption sites which are nearest neighbors to a given site. Davis (1950a) discusses three types of surfaces:

1. A multilinear array of widely spaced rows-

$$
Y=2 \text { and all } q_{i}=1
$$


2. An open-packed array of sites-

3. A close-packed array of sites-

$$
Y=4 \text { and } q_{i}=\frac{z_{i}+1}{2}
$$

$$
Y=6 \text { and } q_{i}=\frac{2 z_{i}+1}{3}
$$

The results for the multilinear array that all $q_{i}=1$ means that in this case equation (4-56) becomes identical with Vanselow's equation. Krishnamoorthy and Overstreet's results $(1950 a)$ indicate that choosing an open-packed array with $q_{i}=$ $\left(z_{i}+1\right) / 2$ leads to somewhat more constant values of $k_{D}$.

\section{Other equations}

Two other types of equations are in use in the current literature. One was advanced by Gapon (1933), and it may be obtained in the following way. Assume that the exchange between $\mathrm{Na}^{+}$and $\mathrm{Ca}^{++}$can be presented by writing

$$
\mathrm{Ca}_{1 / 2} \mathrm{X}+\mathrm{Na}^{+}=\mathrm{Na} X+1 / 2 \mathrm{Ca}^{++}
$$

The suffix $X$ denotes an adsorbed ion. The equilibrium constant is then formulated as

$$
\frac{(\mathrm{Na} X)\left(\mathrm{Ca}^{++}\right)^{1 / 2}}{\left(\mathrm{Ca}_{1 / 2} X\right)\left(\mathrm{Na}^{+}\right)}=k_{G}
$$

where parentheses denote activities. If the activities of adsorbed ions are replaced by the number of moles on the surface and the activities of ions in solution are replaced by concentrations, the result is Gapon's equation:

$$
\frac{n_{\mathrm{Na}^{+}(a d)} \cdot\left(m_{\mathrm{Ca}^{++}}\right)^{1 / 2}}{n_{\mathrm{Ca}^{++}(a d)} \cdot m_{\mathrm{Na}^{+}}}=k_{G}
$$

Still other equations have been used by workers at the U. S. Salinity Laboratory (Richards, 1954). These equations involve the following quantities:

$$
\begin{aligned}
\mathrm{SAR}^{4} & =\text { sodium adsorption ratio } \\
& =\frac{m_{\mathrm{Na}^{+}}}{\left(m_{\mathrm{Mg}^{++}}+m_{\mathrm{Ca}^{++}}\right)^{1 / 2}}
\end{aligned}
$$

Here, the molalities of the solution ions are in millimoles/liter.

$$
\begin{gathered}
\mathrm{ESR}=\text { exchangeable soldium ratio } \\
=\frac{\mathrm{ES}}{\mathrm{CEC}-\mathrm{ES}}
\end{gathered}
$$

Here, ES is the exchangeable sodium (me/100 g) and CEC is the cation exchange capacity (me/100 g). The analyses of a large number of soil samples led to the following empirical regression equation:

$$
\mathrm{ESR}=-0.0126+0.01475(\mathrm{SAR})
$$

More recently, Bower (1959) has published a similar empirical regression equation in which $E S R=\left(\mathrm{Na}^{+}\right)_{\mathrm{ad}} /\left(\mathrm{Ca}^{++}\right)_{\mathrm{ad}}+(\mathrm{Mg})_{\mathrm{ad}}$ is correlated with SAR. The result is

$$
\mathrm{ESR}=.0057+.0173(\mathrm{SAR})
$$

${ }^{4} \mathrm{SAR}$, in (millimoles/liter) ${ }^{1 / 2}$, is related to the $r$ in equation (4-18), which is given in (moles/ liter $)^{1 / 2}$, by: SAR $=31.6 r$. 


\section{APPLICABILITY TO SOIL SYSTEMS}

\section{Introduction}

The two major theoretical approaches to cation exchange are the double-layer theory and the statistical-thermodynamic theory. The mass-action theory is largely formal, and does not present any detailed picture of the state of ions in soils. We will therefore regard Vanselow's equation as a special case of the statistical theory, although it will be frequently convenient to make calculations based on Vanselow's equation.

The double-layer theory assumes that the charge on the surface is continuous and uniform and that the adsorbed ions are nonlocalized. In a general way, these assumptions should find greatest applicability where the distance between the charged sites on the surface is small relative to the radius of the adsorbed ions. Under these conditions, several layers of ions would be required to neutralize the surface. On the other hand, the statistical-thermodynamic model assumes discrete adsorption sites and localization of the adsorbed ions. This model should be applicable where the distance between the adsorption sites is large relative to the size of the ions. Under these conditions one layer of ions would neutralize the surface.

In the case of symmetrical ion exchanges, the double-layer theory predicts a value of unity for all selectivity functions since no specificity is included in the theory. The statistical-thermodynamic approach seems far more informative in such cases. If the selectivity function is a constant, we may infer that the conditions set forth in the model are met. If it is not a constant, one may apparently infer that the specific interaction energy is not fixed.

Greater attention has been given to unsymmetrical exchanges, particularly the $\mathrm{Ca}^{++}-\mathrm{Na}^{+}$exchange. Here, the double-layer equation (4-18) and the statistical thermodynamic equation (4-56) are again in conflict. Data supporting both equation (4-18) (Bolt, 1955a; Bower, 1959; Lagerwerff and Bolt, 1959) and equation (4-56) (Vanselow, 1932; Krishnamoorthy and Overstreet, 1950a; Van der Molen, 1958) have been presented in the literature.

After considering Gapon's equation and the significance of the regression equations, an effort to resolve this conflict will be made.

\section{Double-layer theory and Gapon's equation}

The double-layer equation gives a plot of the ratio of exchangeable monovalent and divalent ions (ESR in the case of sodium) against the reduced ratio $(r)$ that appears to be linear for values of ESR below unity. This has been a basis for the contention that the double-layer exchange equation provides a theoretical basis for Gapon's equation (4-18). While it is a fact that the plots appear to be linear, the double-layer equation actually predicts a considerable dependence of the ratio on $c_{2}$, and this is not consistent with Gapon's equation. In figures 22, 23, and 24, values of $\Gamma_{1} / \Gamma_{2}$ are plotted against $r$ as calculated from equation (4-18) for various surface charge densities. The values of the slopes (Gapon's constant) are summarized in table 1. For a given surface charge density, there is a marked dependence of the slope on $c_{2}$, and it is difficult to see how any support for Gapon's equation is provided by the double-layer equation even at low values of $\Gamma_{1} / \Gamma_{2}$. This same conclusion can be reached from Lagerwerff and Bolt's calculations (1959) at two different values of $c_{2}$. 
TABLE 1

Values of Gapon's Function $\left(k_{G}\right)$ Calculated from Double-Layer Theory for Various Divalent-Ion Concentrations and SurfaceCharge Densities at Low Values of the Reduced Ratio

\begin{tabular}{c|c|c|c}
\hline \hline \multirow{2}{*}{$\begin{array}{c}c_{2} \\
\text { (molality) }\end{array}$} & \multicolumn{2}{|c}{$k_{G}(\text { moles } / \text { liter })^{-1 / 2}$} \\
\cline { 2 - 4 } & $\Gamma=1 \times 10^{-7}$ & $\Gamma=2 \times 10^{-7}$ & $\Gamma=3.5 \times 10^{-7}$ \\
\hline 0 & 1.392 & 0.653 & 0.379 \\
\hline .0025 & 1.123 & 0.580 & 0.348 \\
\hline .025 & 0.792 & 0.473 & 0.302 \\
\hline .25 & 0.404 & 0.304 & 0.219 \\
\hline
\end{tabular}

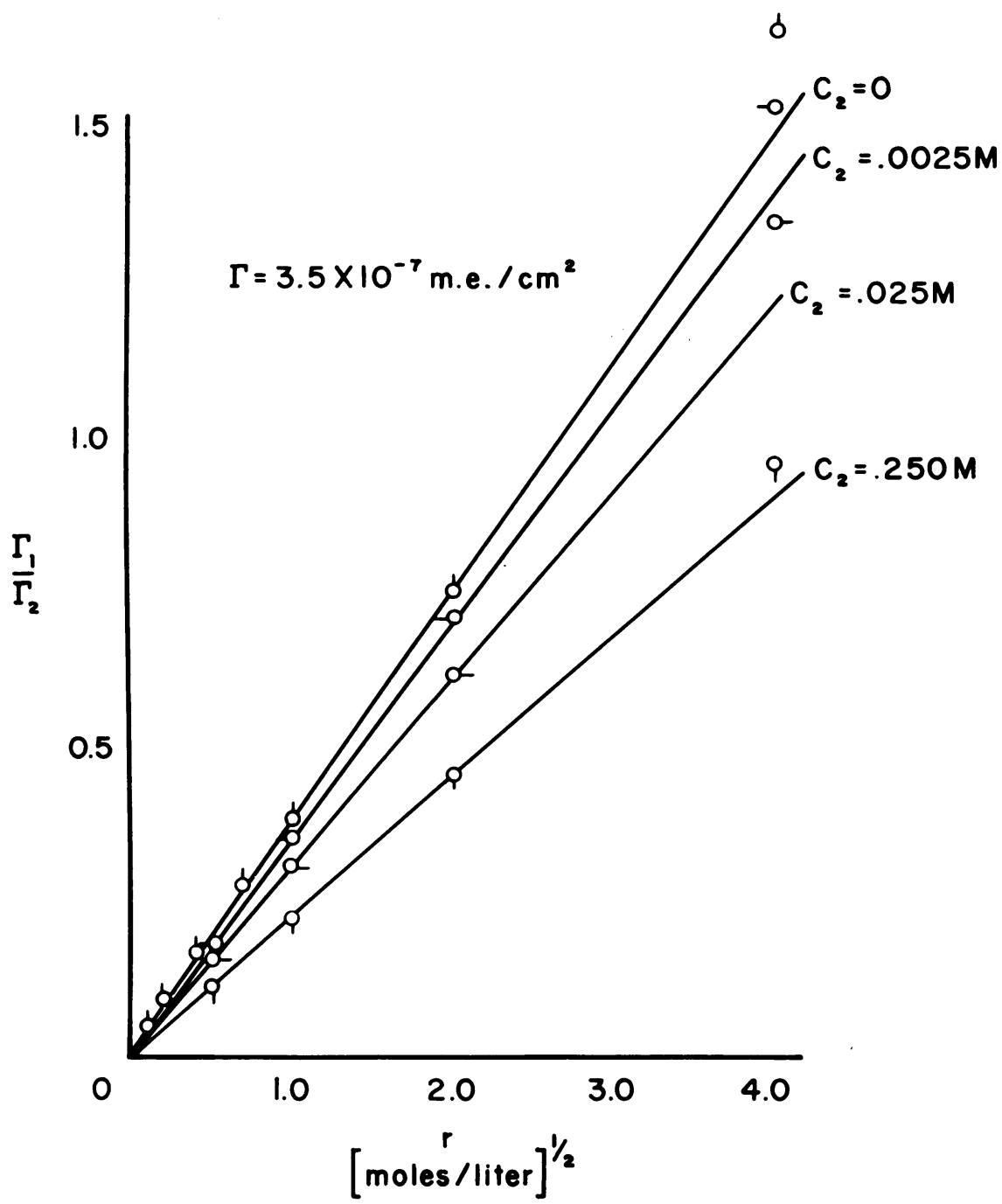

Figure 22. Ratio of adsorbed mono- and divalent ions as a function of the reduced ratio at various divalent ion concentrations according to Gouy theory. 


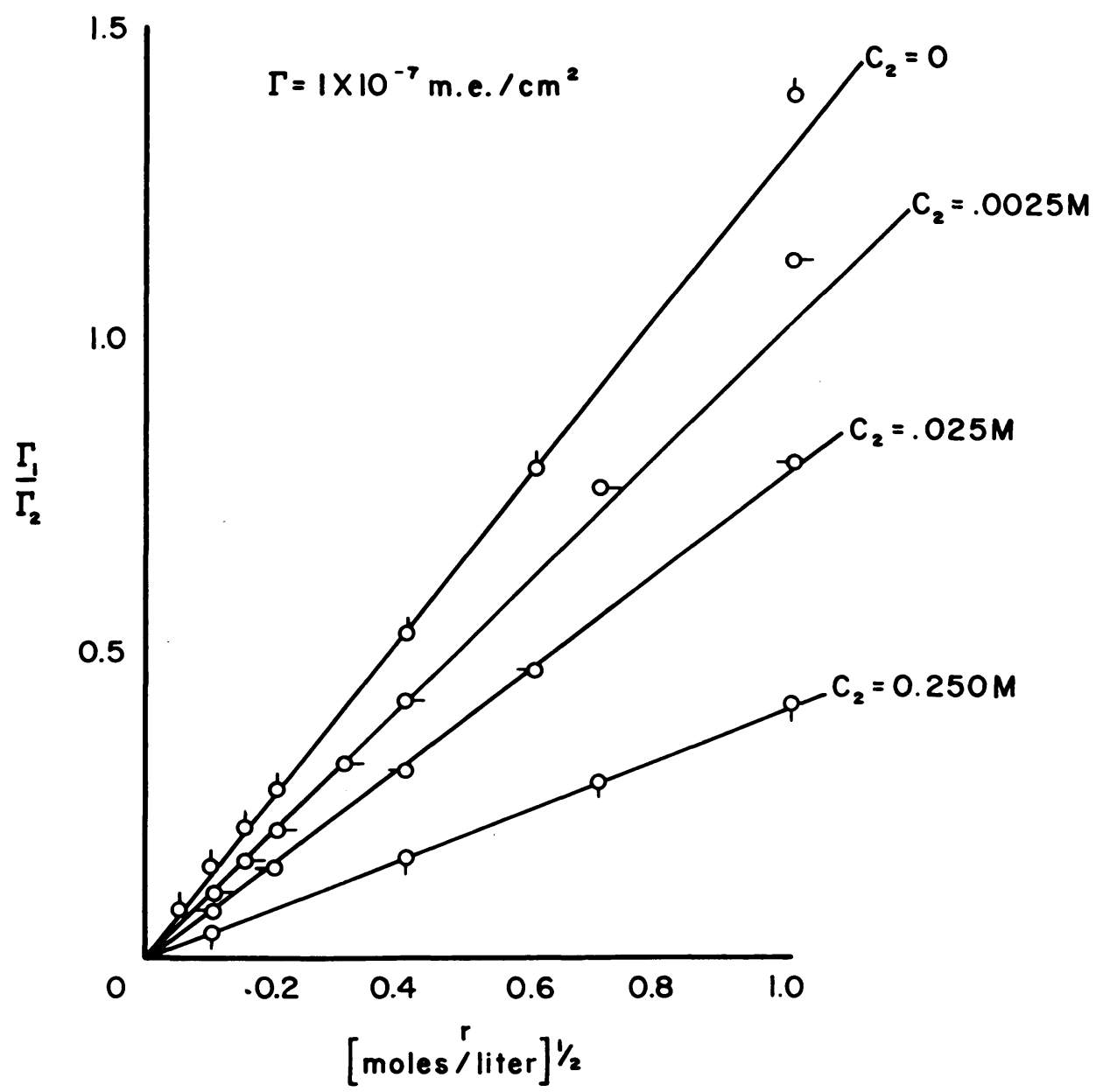

Figure 23. Ratio of adsorbed mono- and divalent ions as a function of the reduced ratio at various divalent ion concentrations according to Gouy theory.

When $\Gamma_{1} / \Gamma_{2}$ exceeds unity, the plots no longer appear to be linear as shown in figures 25,26 , and 27 .

Finally, it should be noted that at low values of the reduced ratio, Vanselow's equation also gives a linear relation between the ESR and $r$, when activity coefficients of solution ions are omitted. To see this, consider Vanselow's equation for a $\mathrm{Na}^{+}-\mathrm{Ca}^{++}$exchange:

$$
\frac{\left[\mathrm{Na}^{+}{ }_{a d}\right]^{2} \cdot\left(\mathrm{Ca}^{++}\right)}{\left(\mathrm{Na}^{+}\right)^{2}\left[\mathrm{Ca}^{++}{ }_{a d}\right]\left[\mathrm{Ca}^{++}{ }_{a d}+\mathrm{Na}^{+}{ }_{a d}\right]}=k_{v}
$$

Here, brackets denote the moles of adsorbed ions and parentheses denote solution concentrations. Let $C$ represent the exchange capacity, so that

and

$$
\mathrm{Na}^{+}{ }_{a d}+2 \mathrm{Ca}^{++}{ }_{a d}=C
$$

$$
r=\frac{\left(\mathrm{Na}^{+}\right)}{\sqrt{\left(\mathrm{Ca}^{++}\right)}}
$$




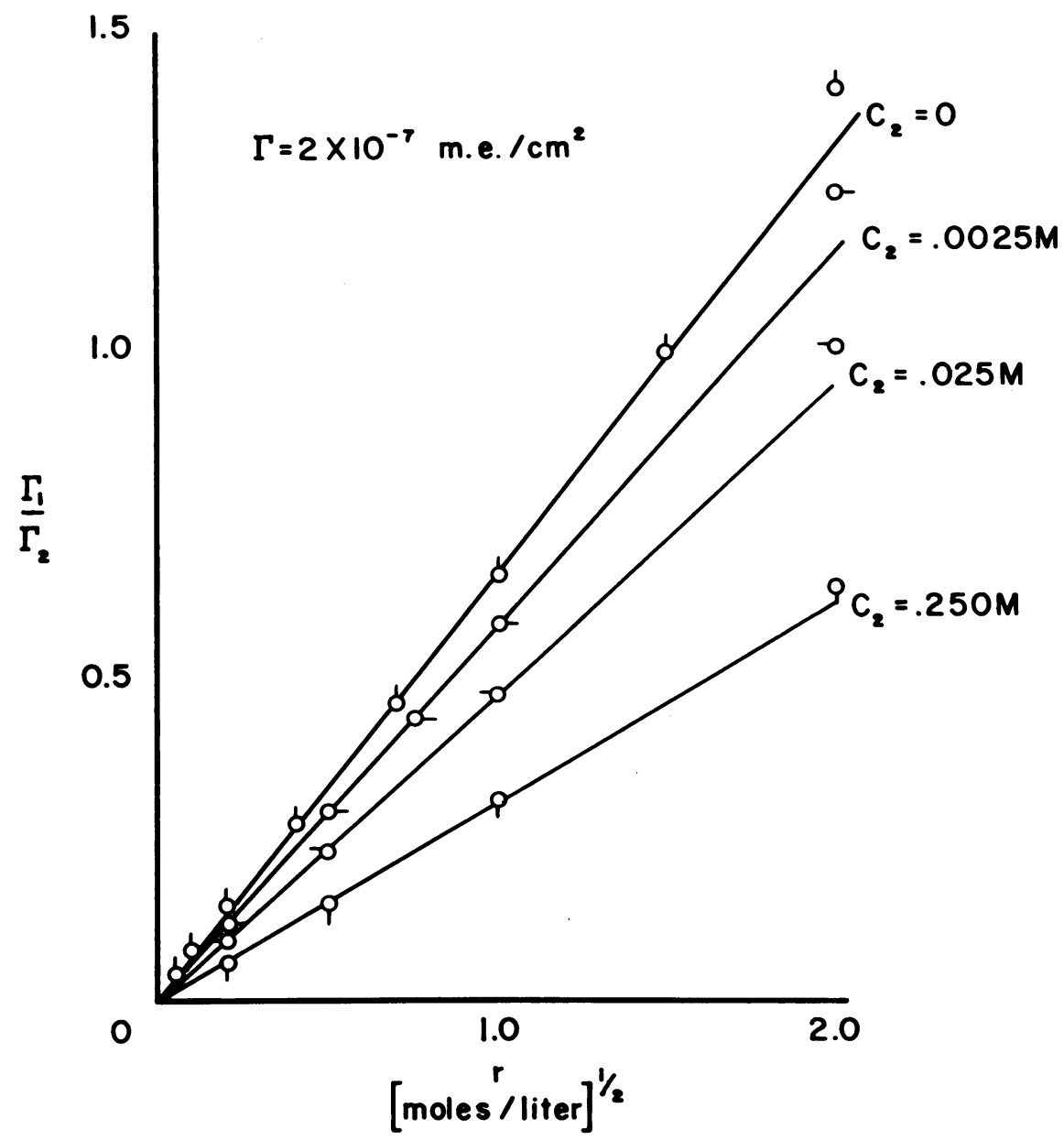

Figure 24. Ratio of adsorbed mono- and divalent ions as a function of the reduced ratio at various divalent ion concentrations according to Gouy theory.

Solving for ESR, we find

For $r \sqrt{k_{v}} \ll 2$, this gives

$$
\mathrm{ESR}=\frac{\left[\mathrm{Na}^{+}{ }_{a d}\right]}{\left[\mathrm{Ca}^{++}{ }_{a d}\right]}=\frac{2 r \sqrt{k_{v}}}{\sqrt{4+k_{v} r^{2}}-r \sqrt{k_{v}}}
$$

$$
\mathrm{ESR} \cong r \sqrt{k_{v}}
$$

Thus, linearity between ESR and $r$ at low values of $r$ is predicted by both the diffuse-layer model and the discrete-phase model.

\section{Regression equations and Gapon's equation}

A number of workers have obtained good correlation coefficients between the ESR of soils and the SAR of their saturation extracts when a large number of samples were analyzed and subjected to statistical analysis (Banerjee, 1959; Richards, 1954). It has been contended that these results support the practical use of Gapon's equation since that equation predicts a linear relationship between the quantities. 


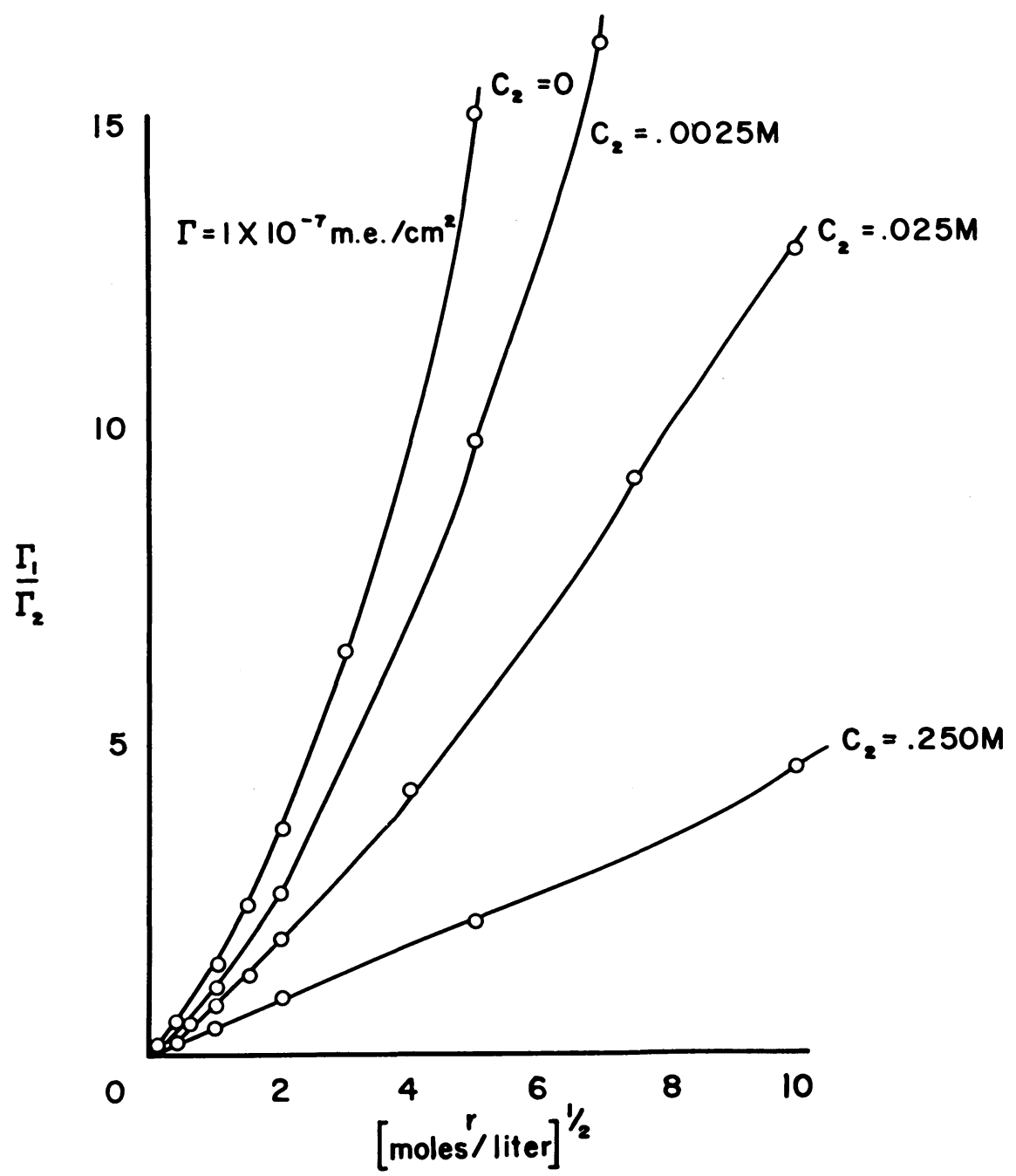

Figure 25. Ratio of adsorbed mono- and divalent ions as a function of the reduced ratio at various divalent ion concentrations according to Gouy theory.

There are two objections to the contention. First, plots of the regression equations do not actually intersect the origin as required by Gapon's equation. Thus, values of $k_{G}$ calculated from the regression equations deviate from constancy at low ESR values even though the discrepancy appears small in the regression plots. In figure 28 the course of $k_{G}$ is plotted using the regression equation (4-60). Since the regression equation leads to variability in $k_{G}$ below about $\mathrm{ESP}=50$, and since validity is claimed for the regression equations only up to $\mathrm{ESP}=50$, it is difficult to accept the conclusion that the high correlation coefficients support Gapon's equation. This difficulty is less marked with the regression equation (4-61) published by Bower, since the regression equation comes nearer the origin.

The second objection, perhaps more serious, involves the nature of "proofs" that use statistics. One may question the procedure of taking a large number of soil 


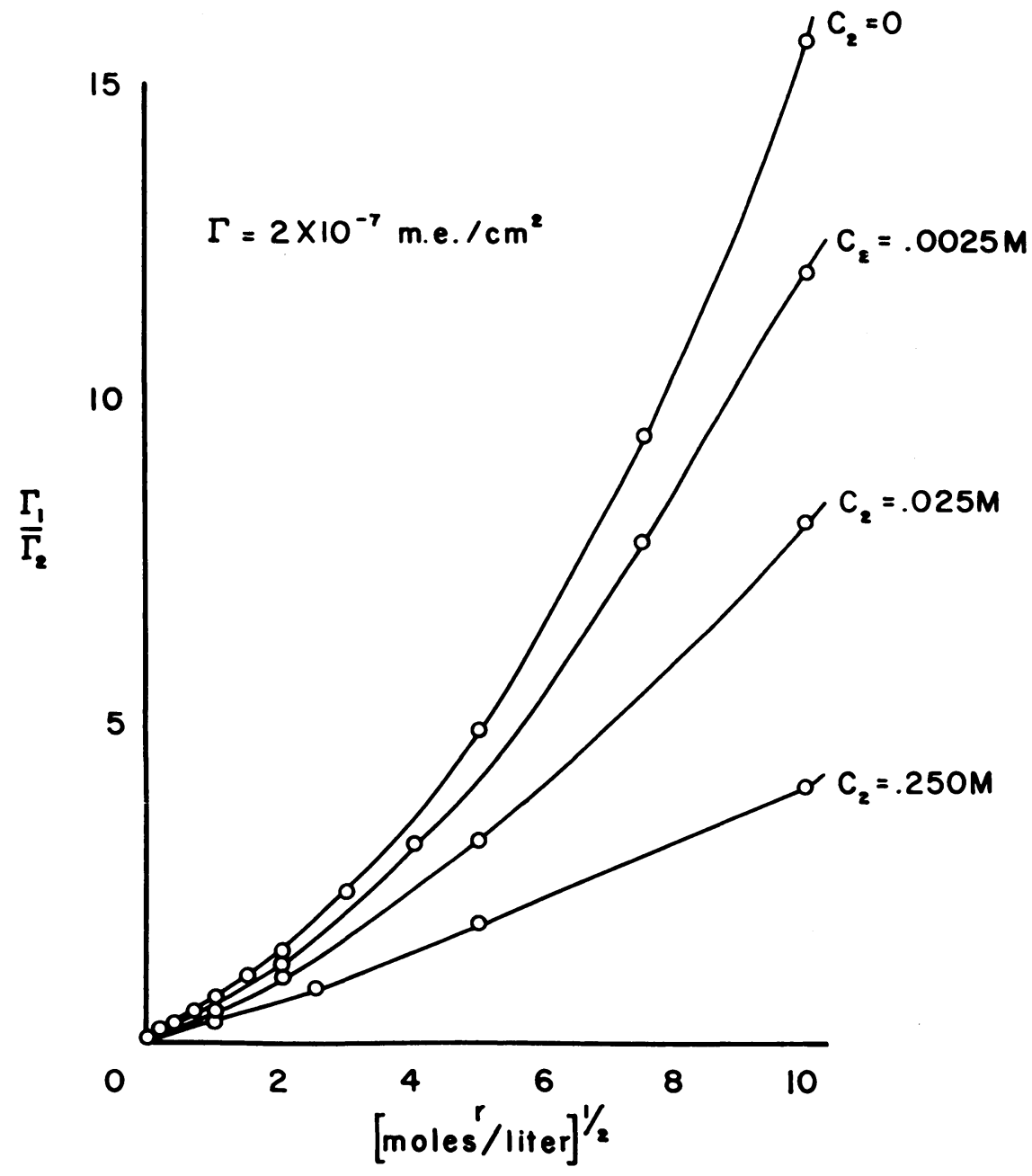

Figure 26. Ratio of adsorbed mono- and divalent ions as a function of the reduced ratio at various divalent ion concentrations according to Gouy theory.

samples, with widely varying properties (including, no doubt, exchange characteristics), and attempting an interpretation of a statistical analysis of the results. The regression equations may have practical significance, but there is doubt that the correlation coefficients have any meaning in relation to Gapon's equation.

As an illustration of this point, a number of workers have obtained high correlation coefficients between ESP and SAR (Lewis and Juve, 1956; Longenecker and Lyerly, 1959) rather than between ESR and SAR. This should not have been possible if Gapon's equation were obeyed by all soils studied and if all soils had the same exchange constant. However, the fact that a statistical correlation between ESP and SAR has been established does not necessarily mean that Gapon's equation is invalidated.

As another example, when Vanselow's equation is used with $k_{v}=0.5$ (moles/ liter $)^{-1}$ (based on Krishnamoorthy and Overstreet's results for Yolo clay, 1950a) 


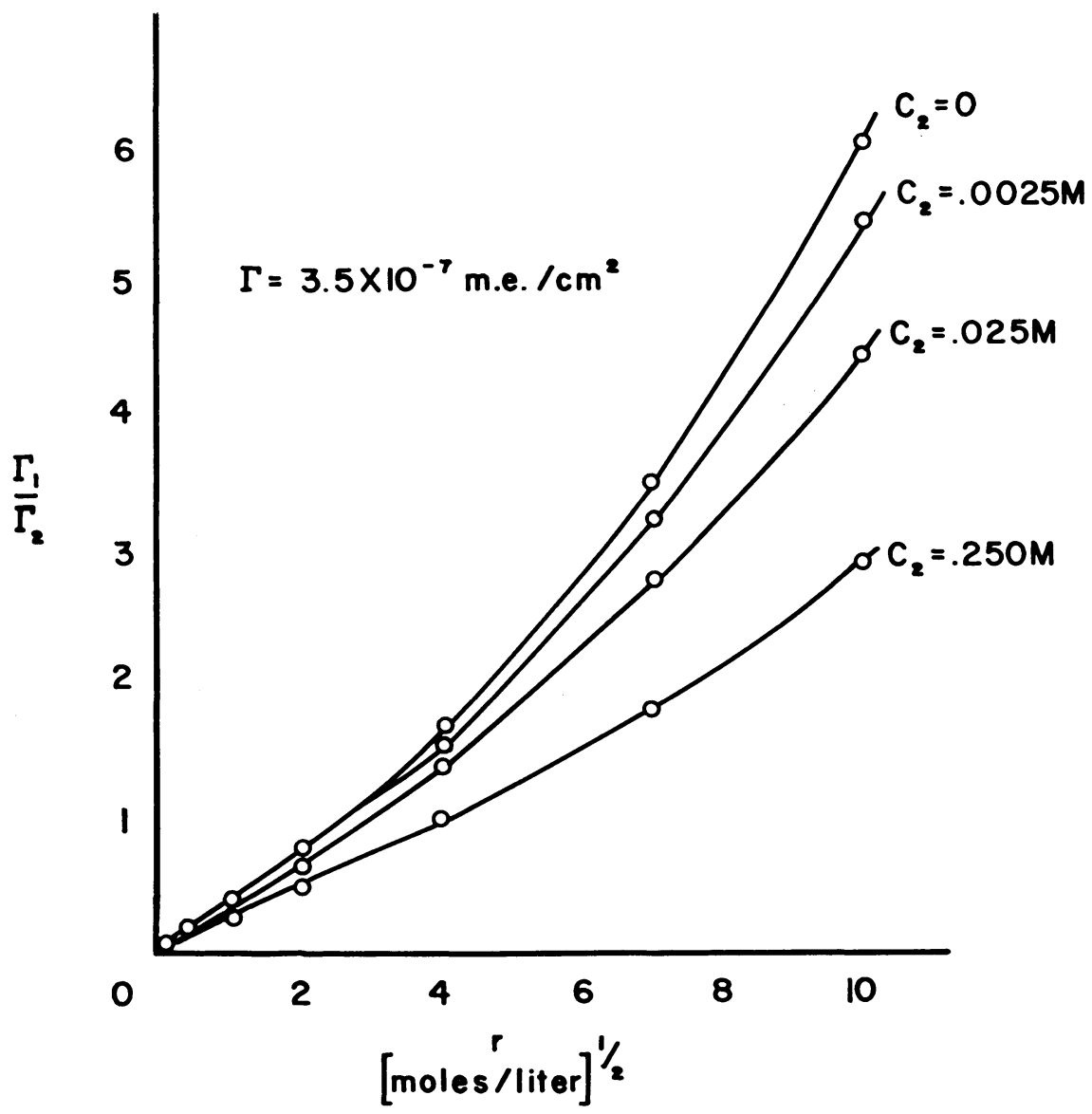

Figure 27. Ratio of adsorbed mono- and divalent ions as a function of the reduced ratio at various divalent ion concentrations according to Gouy theory.

and appropriate activity coefficients for the salts in solution are employed, one can calculate an ESP versus SAR pilot. This has been done for the chloride salts of sodium and calcium and the results are presented in figure 29. The ESP and SAR are highly correlated by a factor of 0.979 . Such a linear relationship between these quantities should not exist in a system obeying Vanselow's equation. Thus, by the method of statistics, results calculated from Vanselow's equation can be used to show that Vanselow's equation is invalid.

Finally, and perhaps most importantly, it must be pointed out that when Gapon's equation has been subjected to direct experimental test, it has been shown to be completely inadequate. The most familiar examples of this are the experiments of Krishnamoorthy and Overstreet (1950a) and of Vanselow (1932). To provide another example, taken at random from the literature, values of $k_{G}$ and $k_{v}{ }^{\prime}$ (where $k_{v}{ }^{\prime}$ is $k_{v}$ uncorrected for activity coefficients) have been calculated from the data of Mattson and Larsson (1946) on the $\mathrm{NH}_{4}{ }^{+}-\mathrm{Ca}^{++}$exchange in bentonite. The value $\sqrt{k_{v}{ }^{\prime}}$ is used because it is more directly comparable with $k_{g}$. Mole fractions were calculated from the data by assuming that the difference between the sum of 


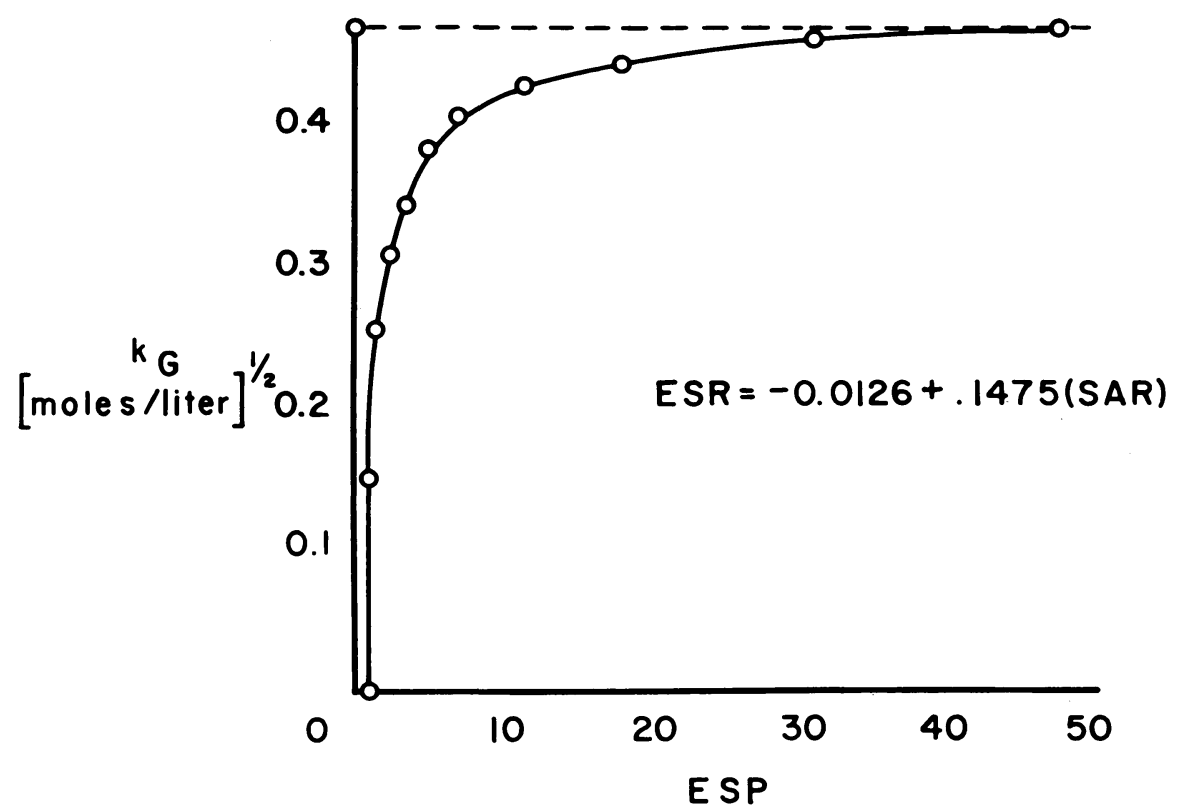

Figure 28. Gapon's ion exchange "constant" calculated from the indicated regression equation.

$\left(\mathrm{NH}_{4}{ }^{+}\right)_{a d}$ and $\left(\mathrm{Ca}^{++}\right)_{a d}$ is adsorbed $\mathrm{H}^{+} .{ }^{5}$ The results are presented in table 2. The regular trend of $k_{G}$ and the random differences in $k_{v}{ }^{\prime}$ are quite typical. Note the wide variation in $\mathrm{pH}$ over which $k_{v}{ }^{\prime}$ is roughly a constant. Krishnamoorthy and Overstreet's data are even more striking.

\section{Statistical-thermodynamic and double-layer equations}

Bolt (1955a), Bower (1959) and Lagerwerff and Bolt (1959) have presented experimental evidence in support of Ericksson's double-layer exchange equation. ${ }^{6}$

5 This assumption is in error if exchangeable aluminum is formed during the course of the experiment.

${ }^{\circ}$ Note added in proof: Pratt and Blair (Hilgardia 33:689, 1962) have added an impressive example of support for the double-layer equation.

TABLE 2

Values of $k_{G}^{*}$ and $\sqrt{{k_{v}}^{*}}$ Calculated from Mattson and Larsson's Data For the $\mathrm{NH}_{4}{ }^{+}-\mathrm{Ca}^{++}$Exchange in Bentonite

\begin{tabular}{|c|c|c|c|c|c|c|}
\hline \multirow{2}{*}{$\mathrm{pH}$} & \multicolumn{2}{|c|}{$\begin{array}{c}\text { Solution concentrations } \\
\text { (normality) }\end{array}$} & \multicolumn{2}{|c|}{$\begin{array}{l}\text { Adsorbed ions } \\
\mathrm{me} / 100 \mathrm{~g}\end{array}$} & \multirow{2}{*}{$k G$} & \multirow{2}{*}{$\sqrt{k_{v}}$} \\
\hline & $\mathrm{NH}_{4}{ }^{+}$ & $\mathrm{Ca}^{++}$ & $\mathrm{NH}_{4}{ }^{+} a d$ & $\mathrm{Ca}^{++}{ }_{a d}$ & & \\
\hline 4.94 & .01005 & .01004 & 10.52 & 64.26 & 1.64 & 2.43 \\
\hline 2.51 & .01017 & .01031 & 6.92 & 30.40 & 2.27 & 2.05 \\
\hline 2.19 & .00997 & .00938 & 6.32 & 26.68 & 2.30 & 1.93 \\
\hline 1.08 & .00995 & .00963 & 5. 12 & 10.84 & 4.66 & 2.36 \\
\hline
\end{tabular}

* Uncorrected for activity coefficients. 


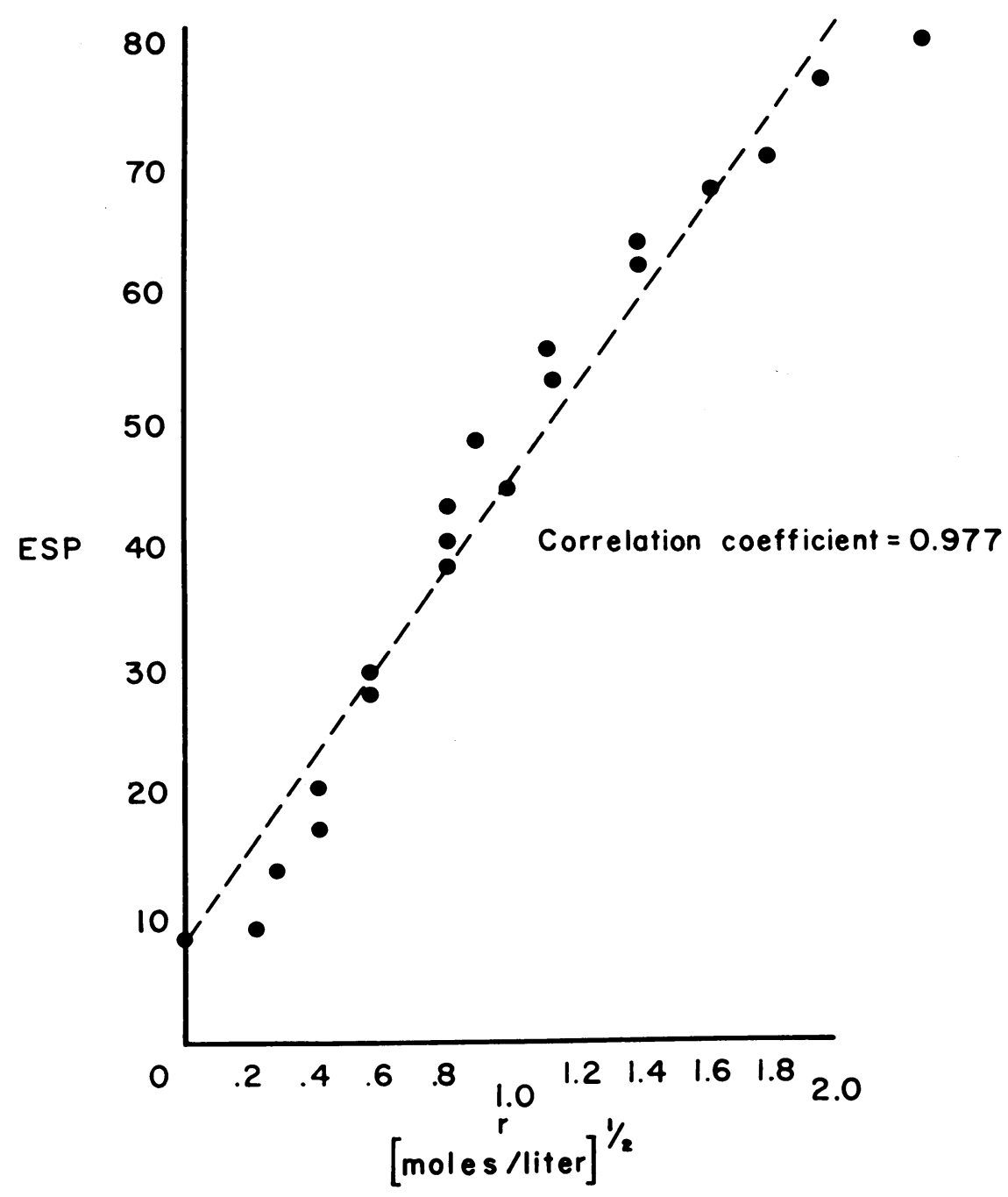

Figure 29. Exchangeable sodium percentage calculated from Vanselow's equation and plotted against the reduced ratio.

Before considering these articles it is important to point out that the double-layer equation predicts, for the case of ion pairs of unequal valency, that the replaceability of the monovalent cation should increase as the surface charge density increases. This is illustrated in figures 30 and 31 , where $\Gamma_{1} / \Gamma$ is plotted against $r$ at various values of $r$, and also in figure 35. In fact, Ericksson has pointed out that if the $\mathrm{NH}_{4}{ }^{+}-\mathrm{Ca}^{++}$exchange constant found by Krishnamoorthy and Overstreet (1950a) is multiplied by the exchange capacity of the clay, one gets rather a good constant. However, this is fortuitous. Table 3 shows the value of the product of the Ca-monovalent ion constant and the exchange capacity $(C)$ for other cations. Only for the $\mathrm{NH}_{4}+-\mathrm{Ca}^{++}$case is the product a constant. In addition, Bolt (1955b) has called attention to the fact that it is the surface charge density, rather than the exchange capacity, which should influence the exchange constant. 


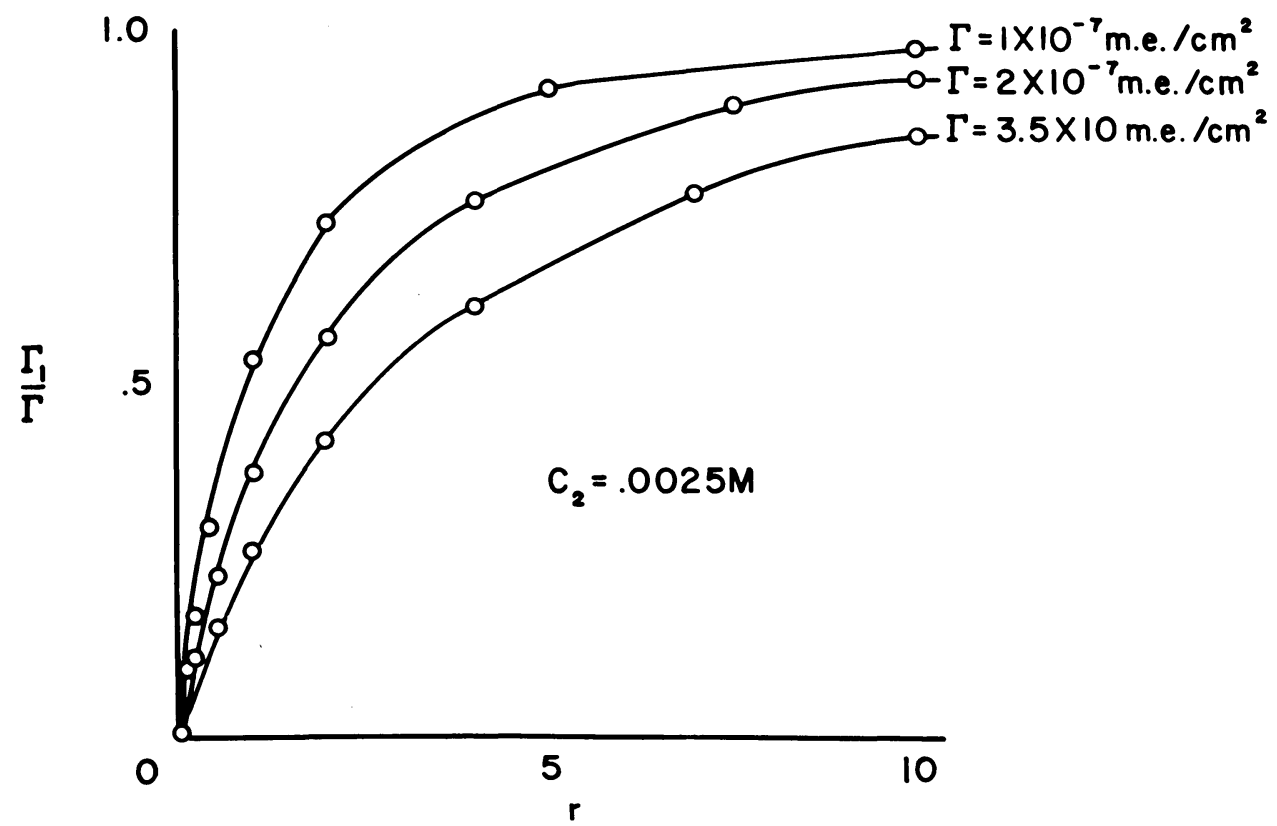

Figure 30. Degree of monovalent ion saturation as a function of the reduced ratio according to Gouy theory.

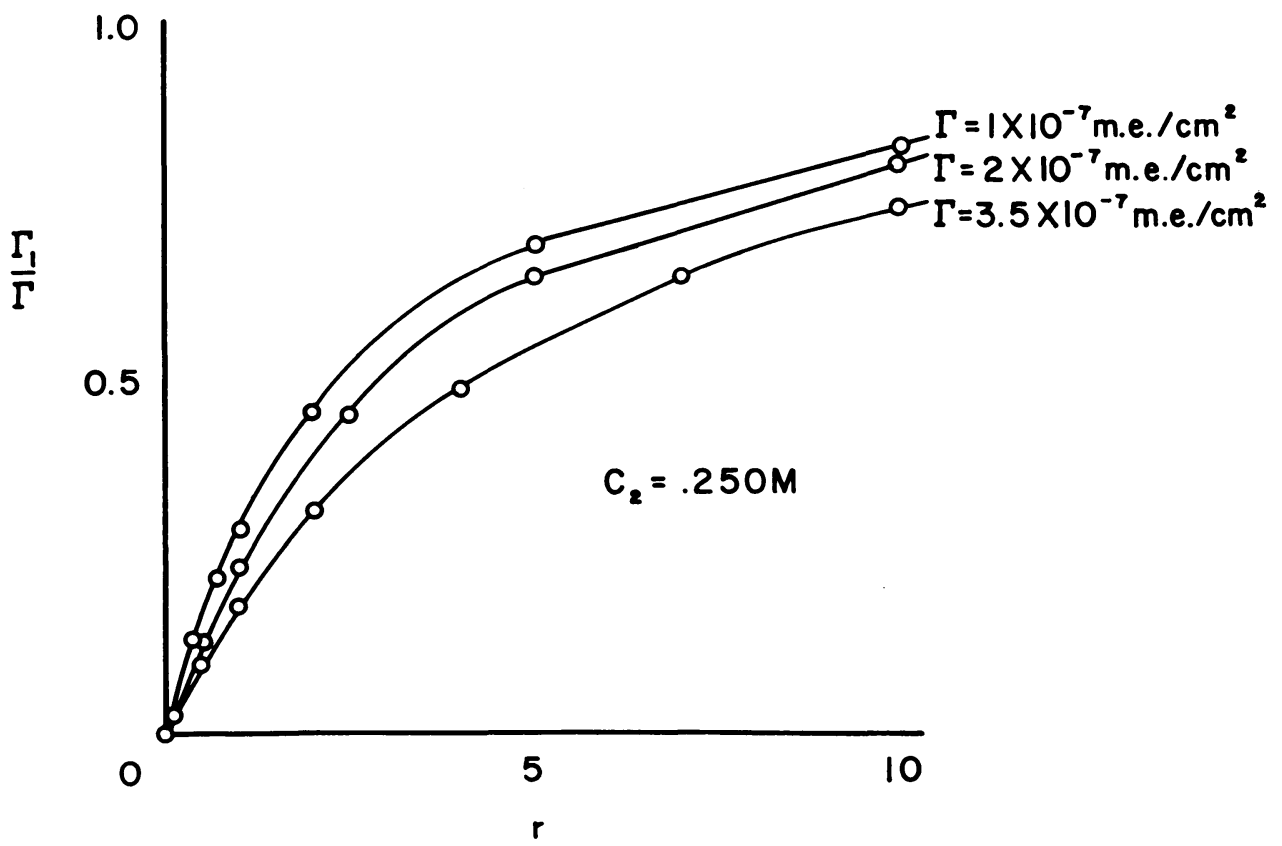

Figure 31. Degree of monovalent ion saturation as a function of the reduced ratio according to Gouy theory. 
TABLE 3

Product of the $\mathrm{Ca}^{++}-$Monovalent Ion-Exchange Constant and the Exchange Capacity $(C)$ for Various Clays

\begin{tabular}{l|c|c|c|c|c|c}
\hline \multicolumn{1}{c|}{ Material } & $\begin{array}{c}C \\
\mathrm{me} / \mathrm{g}\end{array}$ & $k_{\mathrm{Na}^{+}} \cdot C$ & $k_{\mathrm{NH}_{4}+} \cdot C$ & $k_{\mathrm{K}+} \cdot C$ & $k \mathrm{Rb}^{+} \cdot C$ & $k_{\mathrm{Cs}^{+}} \cdot C$ \\
\hline Utah Bentonite & 1.30 & 0.0025 & 0.057 & 0.095 & 0.58 & 12.2 \\
\hline Yolo & 0.60 & 0.0047 & 0.054 & 0.055 & 0.28 & 3.8 \\
\hline Aiken & 0.30 & 0.0021 & 0.055 & 0.019 & 0.90 & $\ldots$ \\
\hline
\end{tabular}

In Bolt's article, the results of a study of the $\mathrm{Na}^{+}-\mathrm{Ca}^{++}$exchange in an illite suspension over a wide range of concentrations are presented. The experimental values of $\Gamma_{1} / \Gamma$ were plotted against $\log r$ and the resulting graph was compared with the theoretical plot from equation (4-18) at $c_{2}=.001$ moles/liter. The data in a general way accord with the theory. However, it is interesting to note that a plot of $\Gamma_{1} / \Gamma$ against $\log r$ as calculated from Vanselow's equation has the same appearance. Figure 32 is plotted with results calculated from Vanselow's equation using $k_{v}=0.5$ (moles/liter) $^{-1}$ but uncorrected for activity coefficients. It seems clear that Vanselow's equation could account for the general shape of the curve obtained by Bolt. However, the double-layer equation does fit the data better than Vanselow's equation, at least in the form where activity coefficients are taken equal to unity.

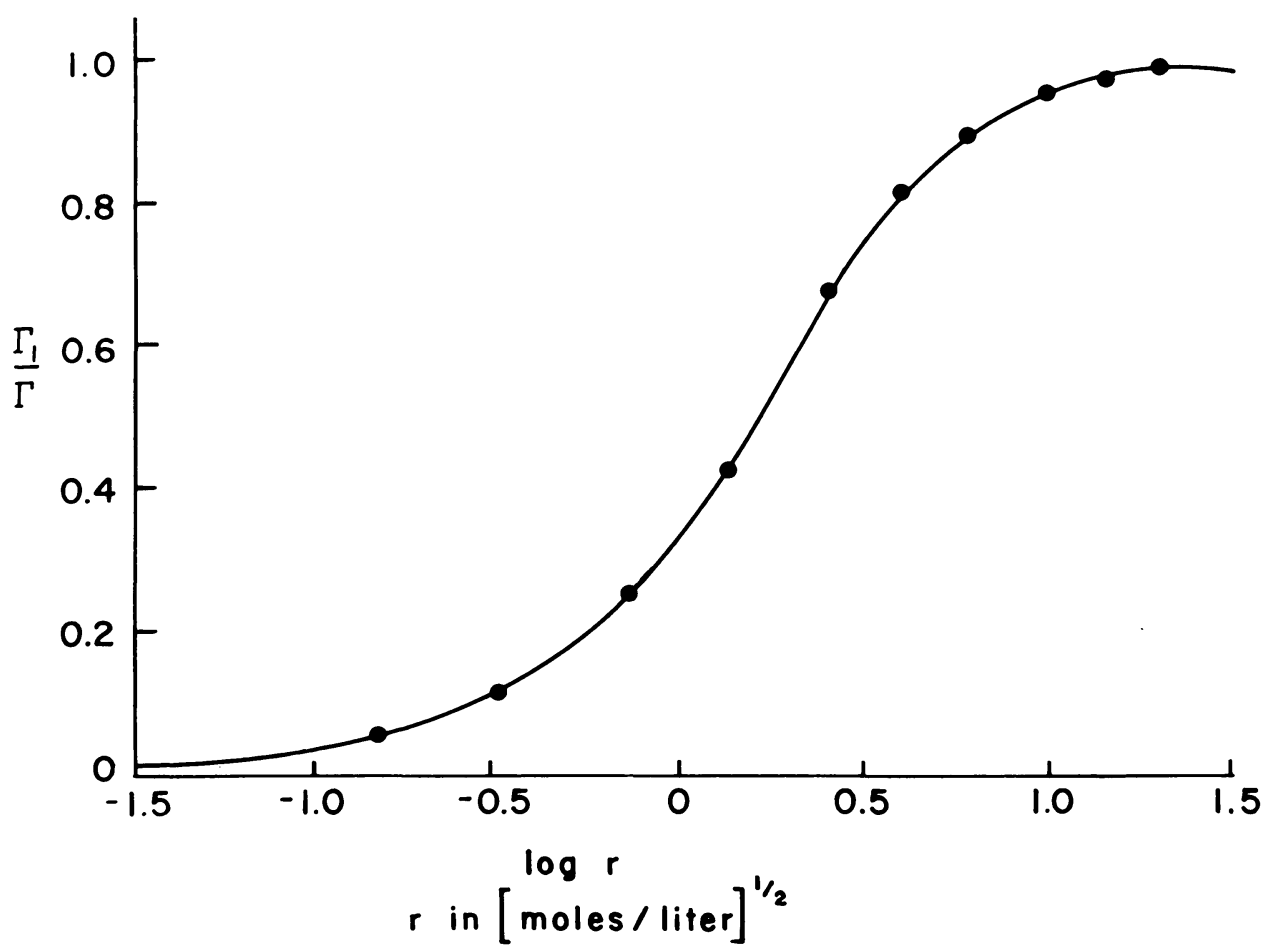

Figure 32. Degree of monovalent ion saturation as a function of the logarithm of the reduced ratio calculated from Vanselow's equation. 


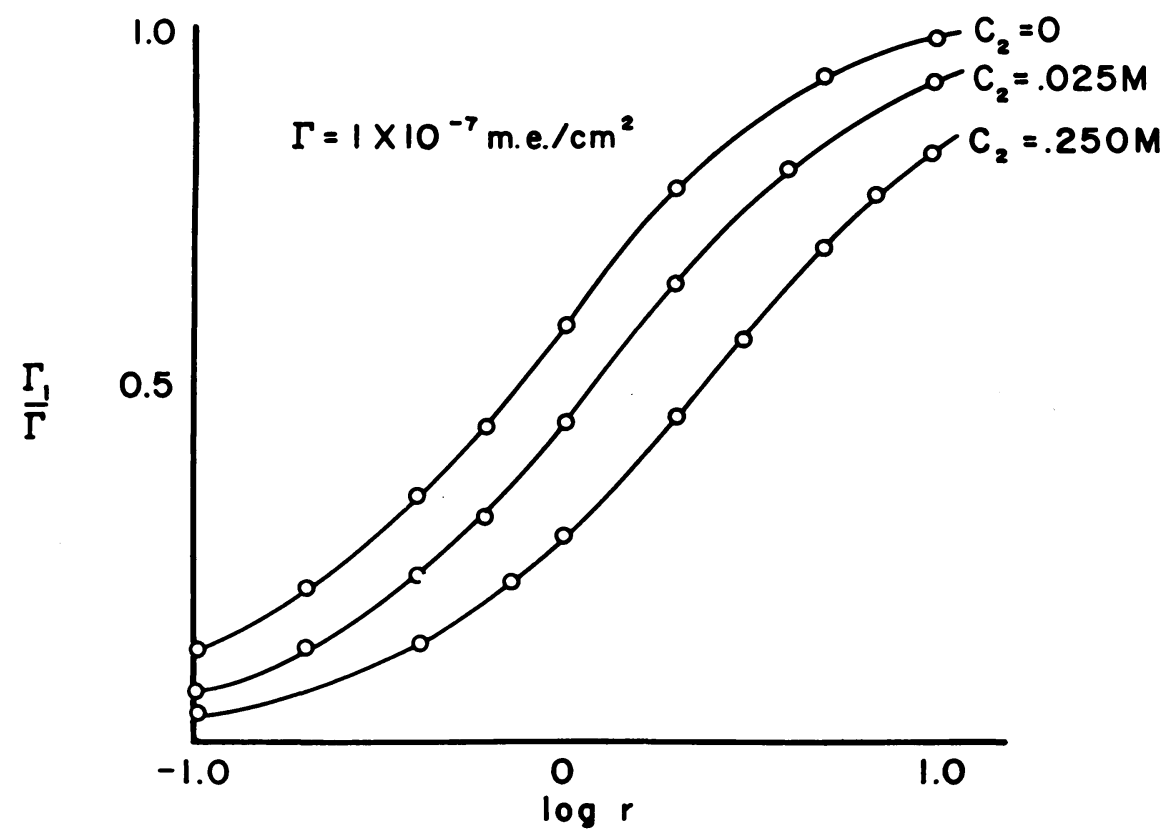

Figure 33. Degree of monovalent ion saturation as a function of the logarithm of the reduced ratio according to Gouy theory.

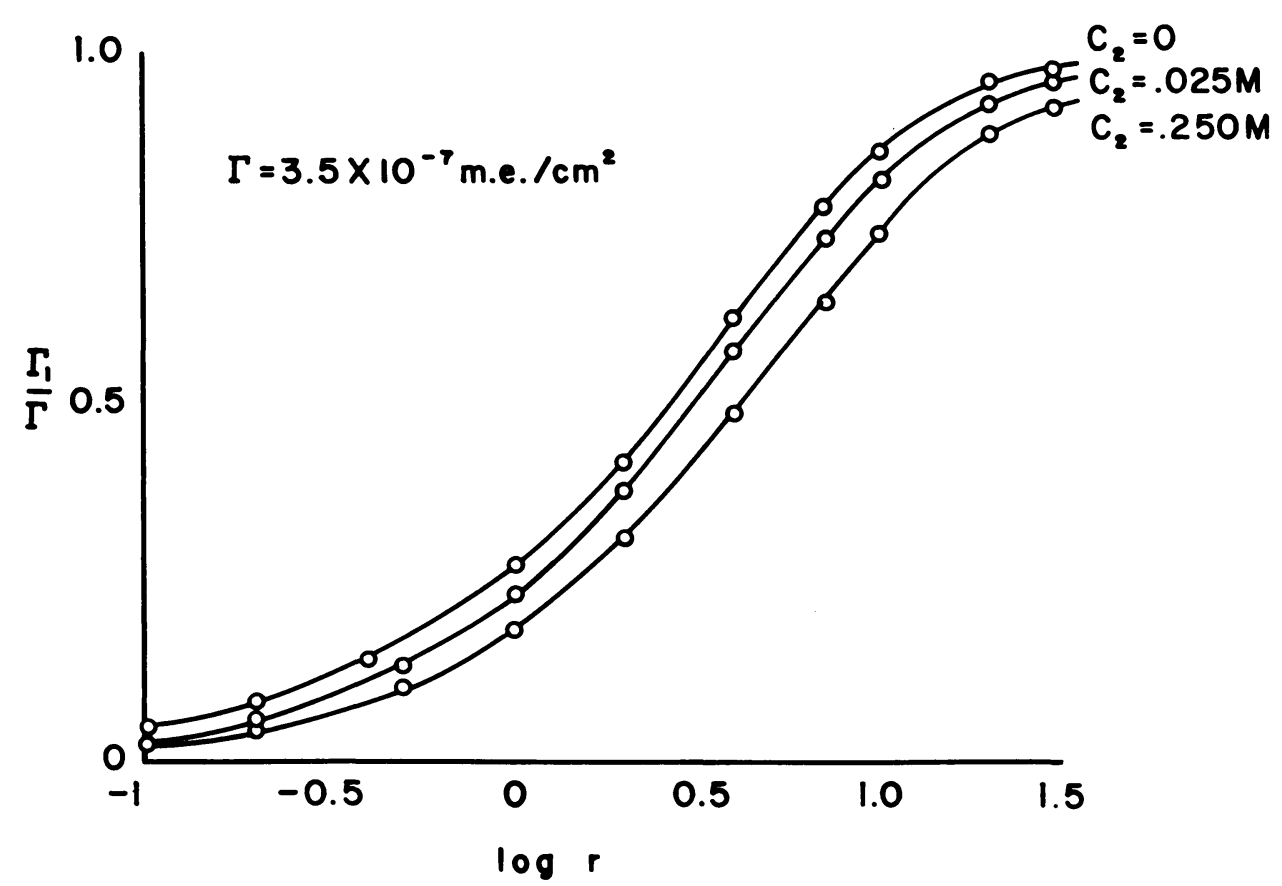

Figure 34. Degree of monovalent ion saturation as a function of the logarithm of the reduced ratio according to Gouy theory. 
It is again instructive to recall that the double-layer equation contains a dependence of $\Gamma_{1} / \Gamma$ on $c_{2}$. This is illustrated in figures 33 and 34 in which values of $\Gamma_{1} / \Gamma$ calculated from equation (4-18) are plotted against $\log r$. For $\Gamma=3.5 \times 10^{-7}$ (the surface charge density used by Bolt), there is a discernible dependence of $\Gamma_{1} / \Gamma$ on $c_{2}$ which is not evident in Bolt's data, although in his experiment $c_{2}$ varied from $.00028 \mathrm{M}$ to $.69 \mathrm{M}$.

Thus, it might be argued that Bolt's data actually support Vanselow's equation, since the Vanselow plot in figure 12 is independent of $c_{2}$.

A strong point in favor of the double-layer equation is the fact that it contains no undetermined parameters, and therefore numerical predictions are possible. For this reason, the numerical agreement between the theory and experiment obtained by Bolt is impressive. Unfortunately, this agreement seems to be partly illusory. The surface charge density for the illite sample used by Bolt was estimated from negative adsorption data by the method of Schofield (1947), and multiplied by a factor of 1.2. This method is based on the assumption of the validity of the double-layer equation, so that when the determined value is used in Eriksson's equation, one would expect the numerical results to be in the right order of magnitude.

More recently Bower (1959) has presented data to show that in soil systems the double-layer exchange equation is followed for the $\mathrm{Na}^{+}-\mathrm{Ca}^{++}$exchange. Bower plots $\Gamma_{1} / \Gamma_{2}$ against $r$ for six soils and clays, and the plots have the same appearance as figures 5 to 7 as required by the double-layer equation. Again, there is fair numerical agreement between theory and experiment. Bower, in agreement with Bolt, concludes that these results rationalize Gapon's equation up to about ESP $=$ $50 \%$.

Bower presents data to show that the exchange relationships for Chino soil are independent of the total cation concentration, by equilibrating the soil with a series of solutions of varying reduced ratio but a constant total cation concentration $(50,100$, and $200 \mathrm{me} / 1)$. The experimental points in plots of $\Gamma_{1} / \Gamma_{2}$ against $r$ all fall on the same curve. Actually, this is contrary to the behavior required by the double-layer theory, since equation (4-18) contains a dependence on $c_{2}$. Table 4 shows the effect of total salt concentration as predicted by equation (4-18) for the

TABLE 4

Effect of Total Salt Concentration on $\Gamma_{1} / \Gamma_{2}$ For Chino Soll ${ }^{*}$ as Calculated from the Double-Layer Exchange Equation

\begin{tabular}{|c|c|c|c|c|c|c|c|}
\hline \multirow{2}{*}{$r$} & \multicolumn{3}{|c|}{$c_{1}+2 c_{2}=0.05 N$} & \multicolumn{3}{|c|}{$c_{1}+2 c_{2}=0.2 N$} & \multirow{2}{*}{$\frac{R D \dagger}{\%}$} \\
\hline & $\begin{array}{c}c_{2} \\
\text { (moles/liter) }\end{array}$ & $\Gamma_{1} / \Gamma$ & $\Gamma_{1} / \Gamma_{2}$ & $\begin{array}{c}c_{2} \\
\text { (moles/liter) }\end{array}$ & $\Gamma_{1} / \Gamma$ & $\Gamma_{1} / \Gamma_{2}$ & \\
\hline 0.25 & .0116 & .099 & .110 & .0675 & .079 & .0856 & 22.2 \\
\hline 0.50 & .00585 & .187 & .230 & .0463 & .154 & .1827 & 20.2 \\
\hline 0.75 & .00330 & .262 & .355 & .0322 & .224 & .289 & 18.6 \\
\hline 1.00 & .00210 & .324 & .480 & .0235 & .288 & .404 & 15.8 \\
\hline 2.00 & .00060 & .513 & 1.050 & .0084 & .484 & .936 & 10.9 \\
\hline
\end{tabular}

${ }^{*} \Gamma=1.76 \times 10^{-7} \mathrm{me} / \mathrm{cm}^{2}$.

$\dagger$ Relative decrease in $\Gamma_{1} / \Gamma_{2}$ due to increased salt concentration. 


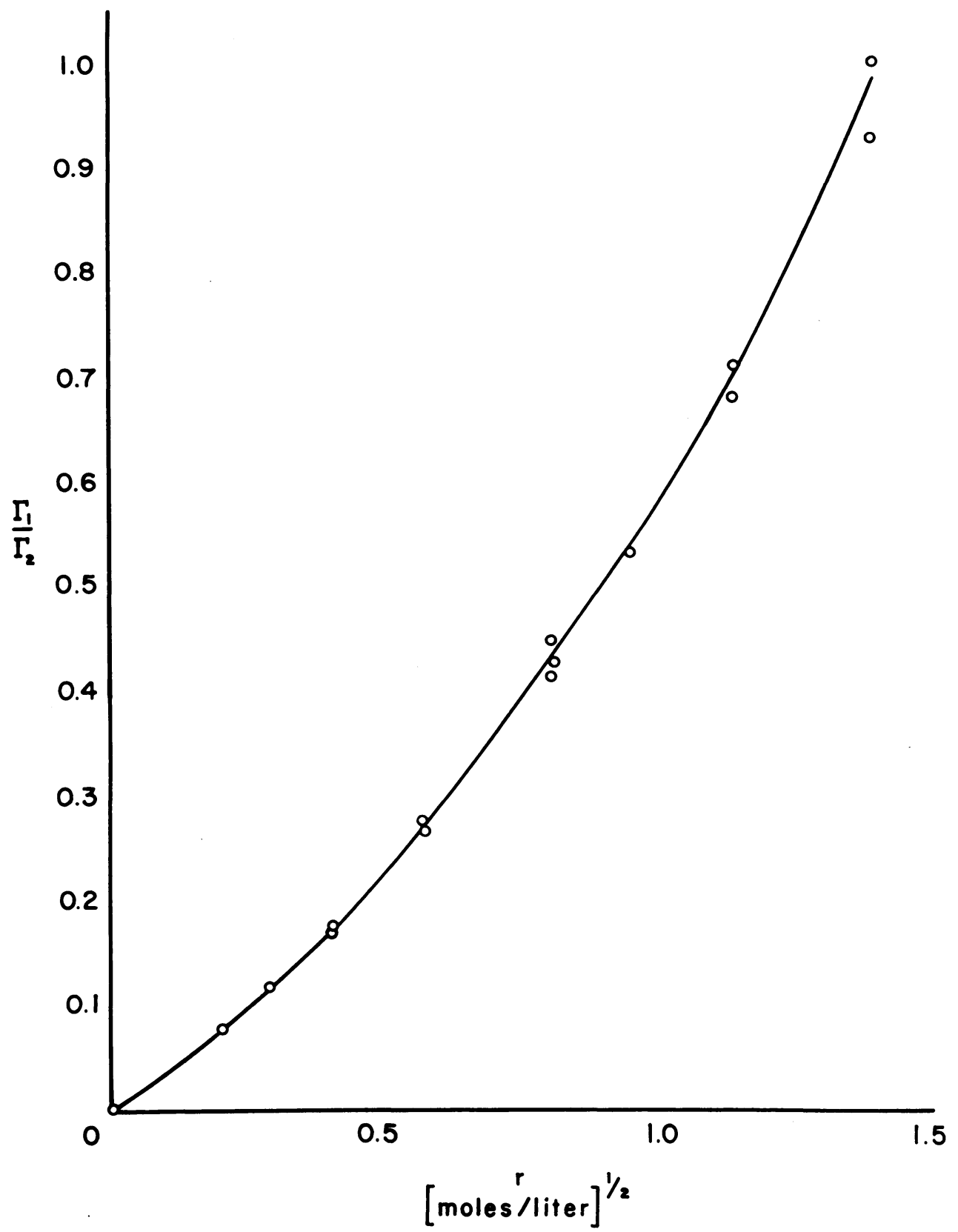

Figure 35. Ratio of adsorbed mono- and divalent ions as a function of the reduced ratio calculated from Vanselow's equation.

range examined experimentally by Bower. It will be noted that in the region for which validity is claimed for Gapon's equation (that is, up to $\Gamma_{1} / \Gamma_{2}=1$ ), the doublelayer equation predicts a notable dependence on total salt concentration between $0.05 \mathrm{~N}$ and $0.2 \mathrm{~N}$. The effect is even more pronounced with soils of lower surface charge density. For example, at $\Gamma=1 \times 10^{-7}$, the relative decrease in $\Gamma_{1} / \Gamma_{2}$ 
between $.05 \mathrm{~N}$ and $0.2 \mathrm{~N}$ is $31 \%$ at $r=0.5$ (compared with $20.2 \%$ in table 3 ). The relative decrease is, of course, also larger when a greater concentration range is selected. Thus, for Chino soil, the relative decrease in going from zero molality to $0.2 \mathrm{~N}$ at $r=0.5$ is $33.7 \%$.

These effects, predicted by the double-layer equation, are not evident in Bower's data. Once again, the fact that the plot is independent of $c_{2}$ could actually be interpreted as evidence for Vanselow's equation. When results calculated from Vanselow's equation are used to plot $\Gamma_{1} / \Gamma_{2}$ against $r$, the curves present an appearance very similar to Bower's plots. This has been done in figure 35, using activity coefficients for chloride salts.

Bower also studied mixtures of $\mathrm{Na}-\mathrm{Ca}-\mathrm{K}$ and $\mathrm{Na}-\mathrm{Ca}-\mathrm{Mg}-\mathrm{K}$ in Chino soil. $\mathrm{He}$ found that the presence of $\mathrm{K}$ had little or no effect on the exchange relationship between $\mathrm{Na}^{+}$and $\mathrm{Ca}^{++}$or between $\mathrm{Na}^{+}$and $\mathrm{Ca}^{++}+\mathrm{Mg}^{++}$. He remarks that "there appears to be no theoretical explanation" for these results. However, Krishnamoorthy and Overstreet $(1950 a)$ also found that the value of a given statistical ion-exchange constant was independent of the amounts of a third ion present, and this is what one would expect on the basis of the two-phase model: the equilibrium constant in equation (4-56) should not be affected by other composition variables. The data of Mattson and Larsson (1946) given above lead to the same conclusion.

Lagerwerff and Bolt (1959) have published data on the Ca-K exchange in illite and montmorillonite suspensions. They conclude that the double-layer equation applies to this exchange in montmorillonite suspensions up to an exchangeable $\mathrm{K}^{+}$ percentage of $25 \%$, but does not apply to illite. They further conclude that the results indicate that Gapon's equation should be of practical use in natural soilwater systems. While the usefulness of an exchange equation is not the question primarily considered here, the experimental results do not provide much confidence in Gapon's equation. For the montmorillonite used by Lagerwerff and Bolt, the value of Gapon's "constant" varied from 0.82 to 2.10 ; the double-layer theory accounts for the observed variation in a qualitative way.

In the three articles just discussed, the conclusions are reached that the monodivalent exchange reaction at low degrees of monovalent ion saturation is controlled mainly by valence and concentration and that specific effects are of a second order which can be treated by multiplying $\Gamma$ by various factors. However, Krishnamoorthy and Overstreet's results (1950a) indicate very pronounced specificities. The constants, in (me/100 cc $)^{-1}$, for the $\mathrm{M}^{+}-\mathrm{Ca}^{++}$exchange when Davis' statistical equation is applied to Utah Bentonite, are:

$$
\begin{array}{ll}
\mathrm{Li}^{+}: & 7.76 \times 10^{-5} \\
\mathrm{Na}^{+}: & 1.95 \times 10^{-3} \\
\mathrm{~K}^{+}: & 7.32 \times 10^{-2} \\
\mathrm{Rb}^{+}: & 0.448 \\
\mathrm{Cs}^{+}: & 9.38
\end{array}
$$

These values indicate that there is pronounced specificity in monovalent-monovalent exchange reactions; double-layer theory does not include these effects in a direct way. 


\section{Hysteresis}

Vanselow's study of cation exchange (1932) revealed that in certain exchanges an effect called hysteresis may be observed. That is, a different value of the equilibrium constant is obtained depending upon the direction of approach to equilibrium. In a recent study of this problem, Tabikh, Barshad, and Overstreet (1960) conclude that hysteresis is not an inherent part of the exchange reaction, but that it is induced by treatments which change the character of the adsorbing surface. In particular, when clay systems are dried, changes are induced which affect the interactions between adsorbed ions and the surface, which in turn affect the selectivity function. The exchange reactions are completely reversible in systems which are always wet.

\section{Exchangeable hydrogen}

Krishnamoorthy and Overstreet (1950a) found very satisfactory exchange constants for all ion-pairs except those involving hydrogen ions. Later, Krishnamoorthy and Overstreet (1950b) were able to obtain exchange constants for $\mathrm{H}^{+}$ by defining a special function of the hydrogen adsorbed to be used in place of the function derived from statistical thermodynamics. The theoretical significance of their procedure is not at present understood.

\section{Summary}

According to the present analysis, support is not provided for Gapon's equation by either double-layer theory, or by experimental regression equations. Furthermore, Gapon's equation generally has been shown to be completely inadequate when subjected to direct experimental test so that there appears to be every reason to abandon this formulation.

The situation is not so definite in comparing the double-layer equation with the statistical-thermodynamic equation. Much of the pertinent data is consistent with either equation, although in the writer's opinion, there are a number of reasons for preferring the statistical-thermodynamic approach.

1) At the high surface charge densities of clay particles and at the salt concentrations encountered in soils, there is good reason to believe that the exchangeable ions are at a distance from the surface which is in the same order as the hydrated radius of the ion. Further, an appreciable fraction of the ions may be held between the layers of expanding lattices in localized positions. In such circumstances, the assumption of point charges and the neglect of ion-ion interactions may constitute an omission of first-order effects. The statistical-mechanical approach should prove more satisfactory in such circumstances.

2) Specific effects are more rationally introduced into statistical theory, and these effects are large.

3) The available data do not appear to exhibit the dependence on $c_{2}$ required by the double-layer theory.

4) The independence of a given exchange relationship from the presence of a third ion cannot be explained in the double-layer theory, whereas it is a direct consequence of statistical theory.

5) Direct experimental tests of the statistical-thermodynamic theory have been very successful. 
It would appear, then, that the first order effects in ion-exchange in soils are best approached on a localized statistical-thermodynamic model, rather than a nonlocalized model. However, Donnan effects and negative adsorption are also observed in these systems. There is every possibility that the localized layer treated by statistical mechanics has a diffuse "tail" so that in dealing with first order cation effects the statistical model is appropriate, while in dealing with first order anion effects, the double-layer model is appropriate. 


\section{Section V \\ THERMODYNAMICS OF WATER IN SOIL SYSTEMS}

\section{INTRODUCTION}

Much greater attention has been given to water in soil systems than to any of the subjects in preceding sections. As a result, there has been an even greater multiplicity of methods of approach and systems of nomenclature. Bolt and Frissel (1960) have systematically summarized the equations which have been presented in the literature. The equations are somewhat bewildering because of their large number and the inconsistency of their nomenclauture.

Two approaches to model systems for the development of the theory of soil water are possible. The first assumes that the soil can be represented as an assemblage of particles forming pores which contain water and air thus comprising a three-phase system. While this seems quite natural, it offers some difficulties for a thermodynamic treatment. For example, in specifying composition variables, the composition of the gaseous phase should be given, although this is often not necessary or convenient. Another difficulty concerns the partial molal volume of water in the system. This quantity is the derivative of the volume of the whole system with respect to the amount of water in the system:

$$
\tilde{V}_{w}=\left(\frac{\partial V}{\partial n_{w}}\right)_{T, P, n_{i}}
$$

where subscript $w$ refers to water. In a three-phase system, this quantity requires careful interpretation. For example, one can imagine an assemblage of sand grains such that when water is added no change in the total volume occurs. However, $\bar{V}_{w}$ is not zero in such a case. Such an addition of water at constant external pressure also involves the expulsion of matter from the gaseous phase so that the constancy of the $n_{i}$ required in the partial derivative is not satisfied. If water were added in such a way that the quantity of matter in the gaseous phase were held constant and the pressure were held constant, a volume increase would in fact occur. If the density of water in the system is essentially the same as pure water, this volume increase would be $\bar{V}_{w}^{\circ} d n_{w}$ where $\bar{V}_{w}^{\circ}$ is the molal volume of pure water. In the case where water is added and air is expelled, the volume change is given as

$$
d V=\sum \sum \bar{V}_{i} d n_{i}
$$

the double summation being over the components and the phases. In the simplest cases, this summation may be zero, although the various values of $\bar{V}_{i}$ are not.

These difficulties might be avoided by defining the soil system to include only the particles and the water. The gaseous phase would thus always be a part of the surroundings, and composition variables for this phase would not be required. If one mole of water were added to a large volume of the system, the volume increase would be approximately that of the added water and thus approximately equal to $\bar{V}_{w}$. However, even greater difficulties are now presented. An accurate determination of the volume of the system becomes difficult. The concept of a saturated soil becomes lost or difficult to define. The process of swelling is difficult to treat. 
In view of these difficulties, the three-phase system will be used in this section. The model will be assumed to be macroscopically homogeneous. Air bubbles will be assumed absent and hysteresis will be ignored. These omissions may be serious, but at present a means of including air bubbles or hysteresis in a thermodynamic treatment does not appear to be available.

\section{NONSWELLING SYSTEMS}

\section{Chemical potential}

Babcock and Overstreet (1955) presented a treatment of soil moisture by selecting temperature, external pressure, solute concentration and water content as independent variables. No specific mention of swelling was made. Bolt and Frissel (1960) as well as Takagi (1959) have pointed out that where swelling occurs, it may be necessary to add a term for the change in the geometry of the particles. For the moment we will defer the question of swelling to a later section.

In an expression for the free energy change of a.model system in which geometry is constant, it is convenient to choose the usual independent variables. Thus,

$$
d G=-S d T+V d P_{e}+\sum \mu_{i} d n_{i}
$$

It should again be emphasized that $P_{e}$ is the pressure external to the system, as discussed in Section I. In the case at hand, this means the pressure in the gaseous phase just outside the solid-liquid-gas system. Just as in the cases of electrostatic field or variable surface area treated in Section I, the use of external pressure is crucial for a clear exposition of the energetics of soil moisture.

The significance of the chemical potential in equation $(5-2)$ is given as

$$
\left(\frac{\partial G}{\partial n_{w}}\right)_{T, P_{e}, n_{i}}=\mu_{w}=\bar{G}_{w}
$$

where $n_{w}$ represents the number of moles of water in the system. The chemical potential is thus defined at constant external pressure. (Geometrical terms are again implicitly constant.) Therefore, if a change in the amount of water in the system produces a change in the curvature of the air-water interface and thus a change in the pressure difference across the interface, the change is nevertheless at constant pressure so long as the external pressure is constant. This treatment is entirely analogous to the treatment of excess pressure in bubbles required in Section I.

One other aspect of equation (5-3) must be considered. It has already been noted that subscript $n_{i}$ requires that matter other than water be constant in the whole system, including the gaseous phase. However, while the matter in the gaseous phase may contribute significantly to the volume of the system, it will be assumed that it does not contribute significantly to the free energy. Therefore, the free energy change with respect to $n_{w}$ represented in equation (5-3) will be taken as independent of the matter in the gaseous phase.

The total differential in the chemical potential for water in soil is now formulated in terms of exactly the same independent variables used in equation (5-2):

$$
d \mu_{w}=\left(\frac{\partial \mu_{w}}{\partial T}\right)_{P_{e, ~} n_{i}} d T+\left(\frac{\partial \mu_{w}}{\partial P_{e}}\right)_{T, n_{i}} d P_{e}+\left(\frac{\partial \mu_{w}}{\partial n_{i}}\right)_{T, P_{e}, n_{i}} d n_{i}
$$


This form of the expression for the chemical potential is essential in order to obtain the correct cross-differentiation formulas for the coefficients of the chemical potential:

$$
\begin{aligned}
& \left(\frac{\partial \mu_{w}}{\partial T}\right)_{P_{e}, n_{i}}=-\left(\frac{\partial S}{\partial n_{w}}\right)_{T, P_{e}, n_{j}}=-\bar{S}_{w} \\
& \left(\frac{\partial \mu_{w}}{\partial P_{e}}\right)_{T, n_{i}}=-\left(\frac{\partial V}{\partial n_{w}}\right)_{T, n_{j}, P_{e}}=-\bar{V}_{w}
\end{aligned}
$$

It is important to note that if other independent variables had been used in equation (5-4) (for example, intensive concentration terms), these formulas could not be obtained.

We now wish to transform equation (5-4) into a form containing intensive composition variables rather than the extensive $n_{i}$. It was shown in Section I that the transformation to mole fraction may be made by using the formula

$$
\left(\frac{\partial \mu_{w}}{\partial n_{i}}\right)_{n_{i}} d n_{i}=\left(\frac{\partial \mu_{w}}{\partial N_{i}}\right)_{n_{i}} d N_{i} \quad\left[T, P_{e} \text { constant }\right]
$$

where $N_{i}$ represents the mole fraction. Thus,

$$
d \mu_{w}=-\bar{S}_{w} d T+\bar{V}_{w} d P_{e}+\sum\left(\frac{\partial \mu_{w}}{\partial N_{i}}\right)_{n_{i}} d N_{i}
$$

As emphasized in Section I, the summation term in equation (5-8) is still over only those chemical species whose mole number changes, not over all mole fractions.

In applying equation (5-8) to water in soil, it is convenient to make a special case of water and introduce the water content $\theta$ as the composition variable. For solute ionic species, it is likewise convenient to introduce the molalities in moles per $1,000 \mathrm{~g}$ of water as the composition variable. Then, for any change at constant mass of soil particles,

$$
d \mu_{w}=-\bar{S}_{w} d T+\bar{V}_{w} d P_{e}+\left(\frac{\partial \mu_{w}}{\partial m_{i}}\right)_{n_{i}, T, P_{e}} d m_{i}+\left(\frac{\partial \mu_{w}}{\partial \theta}\right)_{n_{i}, T, P_{e}} d \theta
$$

Any pressure effects due to changes in surface curvature are here included in the $\partial \mu_{w} / \partial \theta$ term.

It is important to notice two points. As mentioned above, in summing over the solute molalities, the summation is over only those molalities whose mole number changes. Further, in changing the variable from the $n_{i}$ to composition variables, the subscript on the partial coefficients in equation (5-7) or (5-8) does not change. Thus, $\partial \mu_{w} / \partial \theta$ is defined for the change in which the mole number, but not necessarily the composition variable, of all other species is fixed. (For example, if we add water to a binary mixture of water and alcohol, only the change in the mole fraction of the water enters the equation for the chemical potential of either component.) Therefore, for any change in which only the amount of water changes, only the $\theta$ composition term is required in equation (5-8) no matter what the changes in solute concentrations may be.

Equation (5-8) is a macroscopic equation in which each variable is subject to direct and unambiguous measurement. The value of $\mu_{w}$ itself is directly obtainable from the vapor pressure:

$$
\mu_{w}-\mu_{w}^{\circ}=R T \ln p / p^{\circ}
$$


Therefore, if the vapor pressure is a known function of the water content, $\partial \mu_{w} / \partial \theta$ may be directly found. The temperature and external pressure are subject to direct measurement with thermometers and pressure gauges. The water content may be slightly ambiguous since different values are obtained depending on the temperature at which the soil is dried when the water content is determined. This ambiguity can be completely removed by the arbitrary specification of a drying temperature of $105^{\circ} \mathrm{C}$.

Two separate treatments of the ionic molalities in equation (5-8) are possible, but neither involves ambiguity. First, as in the Donnan theory presented in Section II, the bulk concentrations of all the ions in the system may be employed. Second, the molalities of the ions in an equilibrium dialyzate may be used in equation (5-8). To see this, notice that if the soil system is in equilibrium with a dialyzate, the chemical potential of the water in both the soil system and the dialyzate is the same. Furthermore, the value of $d \mu_{w}$ for water in the dialyzate is determined by the $d m_{i}$ term for ions in the dialyzate. This means that the total differential of $\mu_{w}$ for water in the soil is fixed by equation (5-8) if the molalities in the equation are understood to be molalities in the dialyzate. This treatment is by far the most commonly employed and will be the one adopted here.

\section{Pressure terms}

For a three-phase solid-liquid-air system, an external pressure may be applied in different ways. The pressure in the external gaseous phase may be increased, pressure may be applied to solid particles by a piston keeping the gas pressure constant, or both the gas pressure and the pressure on the solid may be increased. Since we wish to work with nonswelling conditions in this section, we will generally have in mind changes in gas pressure since these are least likely to produce any changes in geometry. It should also be noted that throughout this chapter it is assumed that work done on the surroundings during a volume change at constant geometry is $P_{\epsilon} d V$. This will not be the case if conditions are anisotropic. This may be an important limitation in many problems.

When water is added to a saturated system, the volume of the system will increase by the volume of the added water unless the particles have in some way affected the density of the water. In an unsaturated system, the situation is more complex. When water is added, its density may be affected by the particles, there may be an expulsion of air, and the particle geometry may change. All of these will affect the volume change. However, in this section we are not considering changes in geometry and, as previously explained, we interpret $\bar{V}_{w}$ as the volume effect when water is added without the expulsion of air. Thus, $\bar{V}_{w}$ for water in the soil will differ from $\bar{V}_{w}$ for pure water only if the particles have an effect on the density of water.

As the gas pressure on the mass of a confined soil system is increased, the vapor pressure of water increases. We wish to find the pressure increase necessary to increase the vapor pressure, and therefore the chemical potential, up to the value for pure water. If the pressure increase can be effected without change in the geometry of particles and at constant composition, then

$$
d \mu_{w}=\bar{V}_{w} d P_{e}=R T d \ln p
$$


Therefore,

$$
\int_{\mu_{w}\left(P^{\circ}\right)}^{\mu^{\circ}{ }_{w}} d \mu_{w}=\int_{P_{e}^{\circ}}^{\bullet P_{\mathrm{eq}}} \bar{V}_{w} d P_{e}
$$

where $\mu_{w}\left(P^{\circ}\right)$ is the chemical potential of water in the soil at standard pressure and $P_{\text {eq }}$ is the pressure needed to increase $\mu_{w}$ up to $\mu_{w}^{\circ}$. Therefore, if $\bar{V}_{w}$ is independent of $P_{e}$,

Thus,

$$
\mu_{w}\left(P^{\circ}\right)-\mu_{w}^{\circ}=-\bar{V}_{w}\left(P_{\mathrm{eq}}-P^{\circ}\right)=R T \ln \frac{p}{p^{\circ}}
$$

$$
P_{\mathrm{eq}}-P^{\circ}=-\frac{R T}{\bar{V}_{w}} \ln p / p^{\circ}=-\frac{R T}{\bar{V}_{w}} \ln a_{w}
$$

where $a_{w}$ is the activity of the water at standard pressure. This use of the activity of the water was introduced by Taylor (1958). Note that it is not assumed that $\bar{V}_{w}$ is independent of water content, only of pressure.

Suppose now that the external pressure is increased on a soil sample which rests on a membrane with atmospheric pressure on the lower side. The membrane is permeable to salts but not to soil particles, as in the pressure membrane apparatus. The pressure is increased until solution just begins to flow out of the system. Let this pressure be $P_{\mathrm{eq}}{ }^{\prime}$. The water in the soil (I) and in the solution (II) is in equilibrium, so that

$$
\mu_{w}^{\mathrm{I}}=\mu_{w}^{\mathrm{II}}
$$

If, during the increase in pressure, $T, n_{i}, \theta$, and geometry are all constant,

$$
d \mu_{w}{ }^{\mathrm{I}}=\bar{V}_{w}^{\mathrm{I}} d P_{e}
$$

Furthermore, if $\bar{V}_{w}{ }^{\mathrm{I}}$ is constant,

Therefore,

$$
\begin{gathered}
\int_{\mu_{w}^{\mathrm{I}}\left(P^{\circ}\right)}^{\mu_{w} \mathrm{I}\left(P^{\circ}\right)} d \mu_{w} \mathrm{I}=\int_{P^{\circ}}^{P_{\mathrm{eq}}{ }^{\prime}} \bar{V}_{w}{ }^{\mathrm{I}} d P_{e} \\
\mu_{w}{ }^{\mathrm{II}}\left(P^{\circ}\right)-\mu_{w}{ }^{\mathrm{I}}\left(P^{\circ}\right)=\bar{V}_{w}^{\mathrm{I}}\left(P_{\mathrm{eq}}{ }^{\prime}-P^{\circ}\right)
\end{gathered}
$$

$$
P_{\mathrm{eq}}{ }^{\prime}-P^{\circ}=-\frac{R T}{\bar{V}_{w}^{\mathrm{I}}} \ln \frac{a_{w}^{\mathrm{I}}}{a_{w}^{\mathrm{II}}}
$$

Since $P_{\mathrm{eq}}{ }^{\prime}-P^{\circ}$ depends upon the ratio of the activity of the water in the soil at standard pressure, and the activity of water in an equilibrium solution at standard pressure, this pressure difference has been widely interpreted as the measure of the effect of the soil particles on $\mu_{w}$.

The water in a soil system may be equilibrated with a liquid phase in other ways. Suppose, for instance, that the soil system is separated from pure water by a membrane which is permeable only to water. Instead of increasing the pressure on the soil, equilibrium may be established by lowering the pressure of the pure water and allowing the soil to remain at $P^{\circ}$. The necessary pressure lowering is readily found by integrating over the pressure change in the pure water:

$$
\int_{\mu^{\circ}{ }^{\circ}}^{\mu_{w^{\mathrm{I}}}\left(P^{\circ}\right)} d \mu_{w} \mathrm{II}=\int_{P^{\circ}}^{P_{\text {eq }}} \bar{V}_{w}{ }^{\mathrm{II}} d P
$$

$\bar{V}_{w}^{\text {II }}$ can be taken as constant, so that

$$
\mu_{w}{ }^{\mathrm{I}}\left(P^{\circ}\right)-\mu_{w}^{\circ}=\bar{V}_{w}^{\mathrm{II}}\left(P_{\mathrm{eq}}-P^{\circ}\right)
$$


Thus,

$$
P_{\mathrm{eq}}-P^{\mathrm{o}}=\frac{R T}{\bar{V}_{i}^{\mathrm{II}}} \ln a_{w}^{\mathrm{I}}
$$

Alternatively, one may equilibrate the water in the soil with a liquid phase across a membrane permeable to both solutes and water by lowering the pressure in the liquid phase (as in the tensiometer). At equilibrium the liquid phase will have the composition of an extract from the soil. Integrating over the pressure change in the tensiometer,

$$
\int_{\mu_{w} \mathrm{II}\left(P^{\circ}\right)}^{\mu_{w} \mathrm{I}\left(P^{\circ}\right)} d \mu_{w}^{\mathrm{II}}=\int_{P^{\circ}}^{P_{\mathrm{eq}}} \bar{V}_{w}^{\mathrm{II}} d P
$$

Again taking $\bar{V}_{w}^{\text {II }}$ to be constant,

Thus,

$$
\mu_{w}{ }^{\mathrm{I}}\left(P^{\circ}\right)-\mu_{w}{ }^{\mathrm{II}}\left(P^{\circ}\right)=\bar{V}_{w}{ }^{\mathrm{II}}\left(P_{\mathrm{eq}}{ }^{\prime}-P^{\circ}\right)
$$

$$
P_{\mathrm{eq}}^{\prime}-P^{\circ}=\frac{R T}{\bar{V}_{w}^{\mathrm{II}}} \ln \frac{a_{w}^{\mathrm{I}}}{a_{w}^{\mathrm{II}}}
$$

The nomenclature for these pressure terms has been the subject of considerable debate and appears likely to change in the near future. We will note, however, that the negative of the pressure difference in equation (5-22) may be called the "total stress," and the negative of the pressure difference in equation (5-25) may ke called the "tension." Thus,

$$
\begin{aligned}
& \text { Total stress }=-\frac{R T}{\bar{V}_{w}} \frac{T}{\mathrm{II}} \ln a_{w}^{\mathrm{I}} \\
& \text { Tension }=-\frac{R T}{\bar{V}_{w}} \frac{a^{\mathrm{II}}}{\ln } \frac{a_{w}^{\mathrm{I}}}{a_{w}{ }^{\mathrm{II}}}
\end{aligned}
$$

Subtracting equation (5-27) from equation (5-26), we obtain

$$
\text { Total stress }- \text { tension }=-\frac{R T}{\bar{V}_{w}{ }^{\mathrm{II}}} \ln a_{w}{ }^{\mathrm{II}}
$$

The term on the right is the osmotic pressure of the extract, so that

$$
\text { Total stress }=\text { tension }+(\text { osmotic pressure })
$$

This nomenclature has been extensively used in the past, although it is now passing from use. It should be noted that the derivation of equation (5-29) involves only pressures in bulk liquid phases and the partial molal volume of water in such phases. The equation for the pressure-membrane apparatus is not as straightforward since it involves $\bar{V}_{w}$ for water in the soil.

Bolt and Frissel (1960) have pointed out difficulties in equation (5-9). First, the curves for $\mu_{w}$ versus $\theta$ show an appreciable hysteresis. Second, the equation does not contain a geometrical term. They suggest that to get around these difficulties, the total differential in $\mu_{w}$ be expressed in terms of the tension by writing

$$
d \mu_{w}=-\bar{S}_{w}{ }^{\mathrm{I}} d T+\bar{V}_{w}^{\mathrm{I}} d P_{e}+\sum\left(\frac{\partial \mu_{w}}{\partial m_{i}}\right) d m_{i}+\bar{V}_{w}{ }^{\mathrm{II}} d t
$$


in which $t$ is the tension as defined above. This is equivalent to writing

$$
\bar{V}_{w} d t=\left(\frac{\partial \mu_{w}}{\partial \theta}\right) d \theta+\left(\frac{\partial \mu_{w}}{\partial \chi}\right) d \chi
$$

where $\chi$ is an unspecified geometrical term. It is considered that by avoiding the use of a $\theta$ term, in which hysteresis occurs, and a $\chi$ term, which is difficult to measure, the resulting equation (5-30) is more useful in many applications.

Equations (5-30) and (5-31) appear to be based on the assumption that only variations in the $\theta$ or $\chi$ terms will produce a change in the measured tension. However, the equilibrium pressure in the tensiometer will also depend on the temperature and external pressure of the water in the soil. For example, as the external pressure on the soil moisture is increased at constant composition, the equilibrium pressure in the tensiometer will also have to be increased. It follows that equation (5-31) is not the complete expression for the total differential of the tension. The complete differential runs

$$
d t=\left(\frac{\partial t}{\partial T}\right) d T+\left(\frac{\partial t}{\partial P_{e}}\right) d P_{e}+\left(\frac{\partial t}{\partial \theta}\right) d \theta+\left(\frac{\partial t}{\partial \chi}\right) d \chi
$$

No term is included for salt concentration as the effect of adding salt on the chemical potential of soil water and water in the tensiometer is the same at equilibrium, and no change in tension in the tensiometer is required to maintain equilibrium. The coefficients in equation (5-32) will now be found.

For any variation in the properties of the soil, the properties of the water in the tensiometer are changed so as to maintain equilibrium. Therefore,

$$
d \mu_{w}{ }^{\mathrm{I}}=d \mu_{w}{ }^{\mathrm{II}}
$$

and, for the most general variation,

$d \mu_{w}{ }^{\mathrm{I}}=-\bar{S}_{w}{ }^{\mathrm{I}} d T+\bar{V}_{w}{ }^{\mathrm{I}} d P_{e}+\sum\left(\frac{\partial \mu_{w}}{\partial m_{i}}\right) d m_{i}+\left(\frac{\partial \mu_{w}}{\partial \theta}\right) d \theta+\left(\frac{\partial \mu_{w}}{\partial \chi}\right) d \chi$

while

$$
d \mu_{w}{ }^{\mathrm{II}}=-\bar{S}_{w}{ }^{\mathrm{II}} d T+\bar{V}_{w}{ }^{\mathrm{II}} d P^{\mathrm{II}}+\sum\left(\frac{\partial \mu_{w}}{\partial m_{i}}\right) d m_{i}
$$

Using the definition of tension to eliminate $P^{\text {II }}$ from equation $(5-35)$, we find that

$$
d \mu_{w}{ }^{\mathrm{II}}=-\bar{S}_{w}{ }^{\mathrm{II}} d T-\bar{V}_{w}{ }^{\mathrm{II}} d t+\sum\left(\frac{\partial \mu_{w}}{\partial m_{i}}\right) d m_{i}
$$

Equating formulas (5-34) and (5-36), we obtain

$$
-\bar{S}_{w}{ }^{\mathrm{I}} d T+\bar{V}_{w}^{\mathrm{I}} d P_{e}+\left(\frac{\partial \mu_{w}}{\partial \theta}\right) d \theta+\left(\frac{\partial \mu_{w}}{\partial \chi}\right) d \chi=-\bar{S}_{w}{ }^{\mathrm{II}} d T-\bar{V}_{w}{ }^{\mathrm{II}} d t
$$

We at once find that

$$
\begin{aligned}
& \left(\frac{\partial t}{\partial T}\right)_{P_{e}, n_{w}, \chi}=\frac{\bar{S}_{w}^{\mathrm{I}}-\bar{S}_{w}^{\mathrm{II}}}{\bar{V}_{w}^{\mathrm{II}}} \\
& \left(\frac{\partial t}{\partial P_{e}}\right)_{T, n_{w}, \chi}=-\frac{\bar{V}_{w}^{\mathrm{I}}}{\bar{V}_{w}^{\mathrm{II}}} \\
& \left(\frac{\partial t}{\partial \theta}\right)_{T, P_{e}, \chi}=-\frac{1}{\bar{V}_{w}^{\mathrm{II}}}\left(\frac{\partial \mu_{w}}{\partial \theta}\right)
\end{aligned}
$$




$$
\left(\frac{\partial t}{\partial \chi}\right)_{T, P_{\theta,}, n_{w}}=-\frac{1}{\bar{V}_{w}^{I I}}\left(\frac{\partial \mu_{w}}{\partial \chi}\right)
$$

It is of interest to note that if $\bar{S}_{w}{ }^{I}=\bar{S}_{w}{ }^{I I}$, then the effect of temperature is eliminated. However, the partial molal entropies are not likely to be equal (Babcock and Overstreet, 1955; Taylor and Stewart, 1960). Substituting the coefficients into equation (5-32), we obtain

$d t=\left(\frac{\bar{S}_{w}^{\mathrm{I}}-\bar{S}_{w}^{\mathrm{II}}}{\bar{V}_{w}^{\mathrm{II}}}\right) d T-\frac{\bar{V}_{w}^{\mathrm{I}}}{\bar{V}_{w}^{\mathrm{II}}} d P_{e}-\frac{1}{\bar{V}_{w}^{\mathrm{II}}}\left(\frac{\partial \mu_{w}}{\partial \theta}\right) d \theta-\frac{1}{\bar{V}_{w}^{\mathrm{II}}}\left(\frac{\partial \mu_{w}}{\partial \chi}\right) d \chi$

This is the equation for $d t$, rather than equation (5-31).

Multiplying by $-\bar{V}_{w}$ and substituting from equation (5-34), we obtain

$$
d \mu_{w}{ }^{\mathrm{I}}=-\bar{S}_{w}^{\mathrm{II}} d T-\bar{V}_{w}^{\mathrm{II}} d t+\sum\left(\frac{\partial \mu_{w}}{\partial m_{i}}\right) d m_{i}
$$

This is the general relationship involving $\mu_{w}$ and $t$ rather than equation (5-30). As Bolt and Frissel have indicated (1960), equation (5-43) is much simpler to apply in practice than equation (5-34).

\section{SWELLING SYSTEMS}

Any process in which water spontaneously enters a system and increases the volume of the system may be called swelling. The pressure which must be applied to the system to prevent such an entry of water may be called the swelling pressure. Thus, for aqueous solutions, the swelling pressure is simply equal to the osmotic pressure.

When water enters a system, an external pressure on the surroundings may or may not develop. If the system is free to increase in volume indefinitely at atmospheric pressure, then water will continue to enter the system until the water phase with which it is in contact is depleted. During this process, no external pressure will develop. If the system is surrounded by a rigid semipermeable membrane, an external pressure will develop until equilibrium is established with the water phase with which it is in contact. The swelling pressure of a system as defined here is therefore not necessarily an external pressure which the system exerts on its surroundings under some standard conditions of temperature, pressure and composition of the system. Rather, it is the pressure one must apply to the system to prevent the entry of water. The external pressure which may develop in a swelling system is always exerted on a semipermeable membrane or its equivalent.

A difficulty is encountered with swelling colloidal systems or with three-phase solid-liquid-gas systems which may undergo a change in volume at constant pressure. In such cases a volume change may result from either of two distinct effects. The volume may change owing to the simple entry of water, as in aqueous solutions, or it may result from a change in the geometrical arrangement of particles. In many colloidal systems the geometry change may be the major volume effect. In general, energy will be required to change the geometry or packing, and an appropriate term must be included in the free energy equation. Bolt and Frissel (1960) intro- 
duced an unspecified $\chi$ term to care for geometric effects. Such a term may be introduced into the free energy equation as follows:

$$
d G=-S d T+V d P_{e}+\sum \mu_{i} d n_{i}+\left(\frac{\partial G}{\partial \chi}\right)_{T, P_{e}, n_{i}} d \chi
$$

The corresponding expression for the chemical potential, as adapted to soil moisture, is

$$
d \mu_{w}=-\bar{S}_{u} d T+\bar{V}_{w} d P_{e}+\left(\frac{\partial \mu_{w}}{\partial m_{i}}\right) d m_{i}+\left(\frac{\partial \mu_{w}}{\partial \theta}\right) d \theta+\left(\frac{\partial \mu_{w}}{\partial \chi}\right) d \chi
$$

A second difficulty encountered with swelling systems is that the degree of a change in geometry due to pressure change will depend on how the pressure change is applied. In the previous section, changes in gaseous pressure were used on the assumption that this would minimize changes in geometry. However, if pressure is applied to the solid matrix by means of a piston system, significant changes in geometry may occur. Therefore, in considering swelling, an external pressure change will mean a change induced by a piston system with the gaseous phase exposed to the atmosphere. Such pressures are often called "confining pressures."

The coefficient in the $\chi$ term is defined at constant $T, P_{e}$, and all mole numbers. Thus, its measurement depends upon finding a method of determining the effect of a change in particle arrangement at constant temperature, external pressure, and composition. The method of inducing the change in geometry is immaterial. For example, an external pressure may be applied to compress the sample and then removed to restore the original external pressure. In many cases, mechanical manipulation such as stirring may induce the change in geometry. In general, a volume change will be induced by such changes in geometry. If we continue to leave $\chi$ unspecified but select volume units, one may express the $\chi$ coefficient in terms of the volume change:

$$
\left(\frac{\partial \mu_{w}}{\partial \chi}\right)_{T, P e, n_{i}, \theta}=\left(\frac{\partial \mu_{w}}{\partial V}\right)_{T, P_{e}, n_{i}, \theta}
$$

Any method of measuring $\mu_{w}$ as a function of $V$ at constant $T, P_{e}, n_{i}$ and $\theta$ will now give this coefficient. For example, one might measure the vapor pressure change induced by mechanical manipulation of a sample.

We have not yet, however, completed the specification of the $\chi$ term. To attempt this, let us momentarily reconsider the osmometer system described on page 434. The equilibrium condition for water in this system is $\mu_{w}{ }^{\mathrm{I}}=\mu_{w}{ }^{\mathrm{II}}$. If, in equilibrium, an infinitesimal change in pressure is made on each phase in such a way as to maintain equilibrium, we obtain

and

$$
d \mu_{w}^{\mathrm{I}}=d \mu_{w}^{\mathrm{II}}
$$

$$
\bar{V}_{w}^{\mathrm{I}} d P^{\mathrm{I}}=\bar{V}_{w}{ }^{\mathrm{II}} d P^{\mathrm{II}}
$$

It follows that the pressure difference required to maintain equilibrium will be a constant only if $\bar{V}_{w}^{\mathrm{I}}=\bar{V}_{w}^{\mathrm{II}}$. Otherwise,

$$
d\left(P^{\mathrm{I}}-P^{\mathrm{II}}\right)=\left(\frac{\bar{V}_{w}^{\mathrm{II}}}{\bar{V}_{w}^{\mathrm{I}}}-1\right) d P^{\mathrm{II}}
$$


Now consider a soil system containing a tensiometer, with variable confining pressure $P^{\mathrm{I}}$ and an equilibrium pressure $P^{\mathrm{II}}$ in the tensiometer cup:

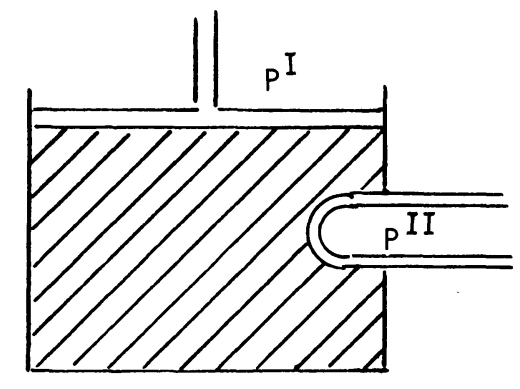

Assume that the system is in equilibrium and that infinitesimal changes are made in $P^{\mathrm{I}}$ and $P^{\mathrm{II}}$ in such a way as to maintain equilibrium. We must include any change in the geometry in the expression for $\mu^{\mathrm{I}}$ :

$$
\begin{gathered}
\bar{V}_{w}^{\mathrm{I}} d P^{\mathrm{I}}+\left(\frac{\partial \mu}{\partial V}\right)_{P} d \chi=\bar{V}_{w}^{\mathrm{II}} d P^{\mathrm{II}} \\
d\left(P^{\mathrm{I}}-P^{\mathrm{II}}\right)=\left(\frac{\bar{V}_{w}^{\mathrm{II}}}{\bar{V}_{w}^{\mathrm{I}}}-1\right) d P^{\mathrm{II}}-\frac{1}{\bar{V}_{w}^{\mathrm{I}}}\left(\frac{\partial \mu}{\partial V}\right)_{P} d \chi
\end{gathered}
$$

Thus, the pressure difference will not in general be constant. In many cases, the first term may be much smaller than the second so that $\Delta \chi$ can be found with equation $(5-51)$ in the approximate form

$$
\Delta \chi=-\int \frac{\bar{V}_{w}^{\mathrm{I}} d\left(P^{\mathrm{I}}-P^{\mathrm{II}}\right)}{(\partial \mu / \partial V)_{P}}
$$

In the experimental study of swelling, the usual practice is to compress the sample in such a way that equilibrium solution may outflow at atmospheric pressure during the external pressure change. Returning briefly to the osmometer analogy, the pressure on the solution in an osmometer may be increased beyond that required for equilibrium so that water is removed to phase II which is at constant pressure. The compression is therefore at constant chemical potential, and we may write for phase I

$$
d \mu_{w}=\bar{V}_{w} d P+\left(\frac{\partial \mu_{w}}{\partial n_{w}}\right) d n_{w}=0
$$

This gives the change in the composition of the solution as the pressure increases. The volume change of the solution during the compression is given as

$$
\begin{aligned}
d V & =\left(\frac{\partial V}{\partial n_{w}}\right)_{P} d n_{w}+\left(\frac{\partial V}{\partial P}\right)_{n_{w}} d P \\
& =\bar{V}_{w} d n_{w}+\left(\frac{\partial V}{\partial P}\right)_{n_{w}} d P \\
\frac{d V}{d n_{w}} & =\bar{V}_{w}+\left(\frac{\partial V}{\partial P}\right)_{n_{w}} \frac{d P}{d n_{w}}
\end{aligned}
$$


To eliminate $d P / d n_{w}$ the equilibrium condition may be written in the form

Thus,

$$
\bar{V}_{w} d P+\left(\partial \mu_{w} / \partial n_{w}\right)_{P} d n_{w}=0
$$

$$
\frac{d P}{d n_{w}}=-\frac{\left(\partial \mu_{w} / \partial n_{w}\right)_{P}}{\bar{V}_{w}}
$$

Substituting equation (5-58) in equation (5-56), we find that

$$
\frac{d V}{d n_{w}}=\bar{V}_{w}-\frac{1}{\bar{V}_{w}}\left(\frac{\partial V}{\partial P}\right)_{n_{w}}\left(\frac{\partial \mu_{w}}{\partial n_{w}}\right)_{P}
$$

This may also be written

$$
\frac{d V}{d n_{w}}=\bar{V}_{w}-\left(\frac{\partial \mu}{\partial V}\right)_{P}\left(\frac{\partial V}{\partial P}\right)_{n_{w}}
$$

Notice that in this case the volume change divided by the change in the number of moles of water is equal to $\bar{V}_{w}$ only if $(\partial V / \partial P)_{n_{w}}$ is zero. For a solution, of course, this term is very small.

Consider now the soil system. If we change the external pressure on the soil keeping the pressure in the tensiometer constant, but maintaining equilibrium by allowing water to flow, then we have the equilibrium condition

$$
d \mu_{w}=\bar{V}_{w} d P+\left(\frac{\partial \mu}{\partial V}\right)_{P, n} d \chi+\left(\frac{\partial \mu}{\partial n}\right)_{P, \chi} d n=0
$$

where $P$ stands for confining pressure and $n$ for moles of water. The expression for the volume change of the soil is

$$
d V=\bar{V}_{w} d n+\left(\frac{\partial V}{\partial P}\right)_{n, \chi} d P+\left(\frac{\partial V}{\partial \chi}\right)_{n, P} d \chi
$$

Combining equations (5-61) and (5-62), we find

$$
\frac{d V}{d n}=\bar{V}_{w}-\frac{1}{\bar{V}_{w}}\left(\frac{\partial V}{\partial P}\right)_{n, \chi}\left[\left(\frac{\partial \mu}{\partial V}\right)_{P, n}\left(\frac{d \chi}{d n}\right)+\left(\frac{\partial \mu}{\partial n}\right)_{P, \chi}\right]+\left(\frac{\partial V}{\partial \chi}\right)_{n, P} \frac{d \chi}{d n}
$$

The total change in volume with $n_{w}$ is thus a complex function equal to $\bar{V}_{w}$ only if such coefficients as $(\partial V / \partial P)_{n, \chi}$ and $(\partial V / \partial \chi)_{n, P}$ are zero. Anderson and Low (1958) measured $d V / d n$ in an experiment of this type and set this derivative equal to $\bar{V}_{w}$. If equation (5-63) is correct, the results will require reinterpretation. It may be noted again that $\bar{V}_{w}$ is a partial derivative at constant pressure.

Taylor and Box (1961) studied the compression of unsaturated soils by measuring the change in the bulk density with a confining pressure and with the gaseous phase open to the atmosphere. This constant water content process is represented as a function of bulk density only. According to the theory above, one should write a two-term equation for the chemical potential change in this case:

$$
d \mu_{w}=\bar{V}_{w} d P_{e}+\left(\frac{\partial \mu_{w}}{\partial V}\right) d \chi
$$

Taylor and Box, however, write

where $\rho_{b}$ is the bulk density.

$$
d \mu_{w}=\left(\frac{\partial \mu_{w}}{\partial \rho_{b}}\right) d \rho_{b}
$$


The use of bulk density is convenient in that it is easily measured. However, it leads to other complications. The bulk density is defined as

$$
\rho_{b}=\frac{m}{V}
$$

where $m$ is the mass. Then,

$$
\left(\frac{\partial \rho_{b}}{\partial V}\right)_{P, n}=-\frac{m}{V^{2}}
$$

Therefore,

$$
\left(\frac{\partial \rho_{b}}{\partial \chi}\right)_{P, n}=-\frac{m}{V^{2}}\left(\frac{\partial V}{\partial \chi}\right)_{P, n}
$$

Using equation $(5-46)$ to find $(\partial V / \partial \chi)_{P, n}=1$, we obtain

$$
\left(\frac{\partial \rho_{b}}{\partial \chi}\right)_{P, n}=-\frac{m}{V^{2}}
$$

The $\partial \rho_{b} / \partial \chi$ term is thus more complex than $\partial V / \partial \chi=1$.

Collis-George (1961) has indicated that the introduction of additional terms, such as a geometrical term or a bulk-volume term, for swelling systems can be avoided by defining the moisture content $(\theta)$ term on a volume basis. However, in the view of the present writer, an independent variable for geometry is required. This is shown by the fact that the volume of a soil system, and hence its geometry, can be changed at constant water content. An additional difficulty in the use of the volume of water in soil is that in cases where the particles affect the density of water, an accurate measure of the water volume is very difficult.

\section{RELATION BETWEEN THERMODYNAMIC AND MECHANICAL TREATMENTS}

Day and Forsythe (1962) have presented a treatment of unsaturated soil systems based on the mechanical principle of virtual work. This treatment has been the basis for experiments of exceptional interest, and an effort will be made here to provide a thermodynamic analysis of their derivation.

The usual variables selected in chemical thermodynamics are the pressure, temperature and mole numbers. Thus, in equation (5-44) the Gibbs free energy is expressed as a function of these variables with a $\chi$ term added for swelling systems. This leads to the complex equation (5-63) for the relation between the volume and number of moles of water in a swelling system.

The principle of virtual work equation formulated by Day and Forsythe can be derived by a similar procedure using the Hemholtz free energy, rather than the Gibbs free energy. Consider the following diagram due to Forsythe (1962).

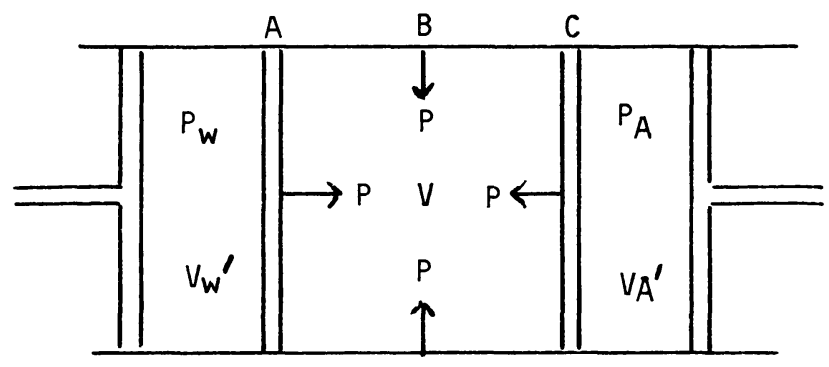


The piston compartment on the left contains water, that on the right contains air, and chamber $B$ in the middle contains soil. Plate $A$ is permeable to water but not to soil and plate $C$ is permeable to air but not soil. The pressures and volumes are as indicated and $P$ is the confining pressure. The whole system is in equilibrium and we may therefore apply the equilibrium condition that for any small displacement at constant temperature and total volume (all three phases) the change in the Hemholtz free energy is zero. (This follows directly from the Second Law, although it is not derived in Section I.) The Hemholtz free energy, $F$, is defined as

$$
F=E-T S
$$

Differentiating and combining with equation (1-33), we find that

$$
d F=-S d T-P d V+\sum \mu_{i} d n_{i}
$$

For a swelling soil system, we may write

$$
d F=-S d T-P d V+\sum \mu_{i} d n_{i}+\left(\frac{\partial F}{\partial \chi}\right) d \chi
$$

Therefore, the total Hemholtz free energy change for the system in the diagram is

$$
d F=-P_{w} d V_{w}^{\prime}-P d V-P_{A} d V_{A}^{\prime}+\sum \mu_{i} d n_{i}+\left(\frac{\partial F}{\partial \chi}\right) d \chi
$$

Now, as previously argued, if the particles have had no influence on the density of water in the soil system, then

$$
d V_{w}=\bar{V}_{w} d n_{w}
$$

where $V_{w}$ is the volume of water in the soil. Thus,

$$
V_{w}^{\prime}+V_{w}=\text { constant }
$$

Further, since it is highly unlikely that there would be a pressure difference between the air in the soil and the air in the gas compartment,

$$
V_{A}{ }^{\prime}+V_{A}=\text { constant }
$$

where $V_{A}$ is the volume of air in the soil. It follows that

$$
\begin{aligned}
& d V_{w}{ }^{\prime}=-d V_{w} \\
& d V_{A}{ }^{\prime}=-d V_{A}
\end{aligned}
$$

Substituting these equations in equation (5-73), we obtain

$$
d F=P_{w} d V_{w}-P d V+P_{A} d V_{A}+\sum \mu_{i} d n_{i}+\left(\frac{\partial F}{\partial \chi}\right) d \chi
$$

It will be recalled that the expression for the chemical potential could be simplified by introducing a tension term which eliminated the $\theta$ and $\chi$ terms. A similar device may be employed for equation (5-79). A quantity $F_{i}$ is defined as

Then,

$$
d F_{i}=\sum \mu_{i} d n_{i}+\left(\frac{\partial F}{\partial \chi}\right) d \chi
$$

$$
d F=P_{w} d V_{w}-P d V+P_{A} d V_{A}+d F_{i}
$$


We now set $d F=0$ at constant temperature and total volume as the equilibrium condition and find

$$
P_{w} d V_{w}-P d V+P_{A} d V_{A}+d F_{i}=0
$$

or

$$
-P_{w} d V_{w}+P d V-P_{A} d V_{A}-d F_{i}=0
$$

Day and Forsythe (in press) write the principle of virtual work in the form

$$
-P_{w} d V_{w}+P d V-P_{A} d V_{A}-\pi d V_{w}=0
$$

where $\pi$ is "the work done by the internal forces of the system per unit volume of water absorbed. . . " Comparing equations (5-83) and (5-84), we obtain

$$
d F_{i}=\pi d V_{w}
$$

This shows the equivalence of the thermodynamic and mechanical treatments provided that it is assumed that $\bar{V}_{w}$ is independent of water content. The significance of the quantity $\pi$ will next be investigated. Substituting equation (5-85) in equation (5-81), we find that

$$
\begin{aligned}
d F & =P_{w} d V_{w}-P d V+P_{A} d V_{A}+\pi d V_{w} \\
& =\left(P_{w}+\pi\right) \dot{d} V_{w} \quad\left(V, V_{A} \text { constant }\right)
\end{aligned}
$$

or

$$
\left(\frac{\partial F}{\partial V_{w}}\right)_{V, V_{A}}=P_{w}+\pi
$$

These equations present a situation which is parallel with the treatment of gravitational fields and electrochemical systems in Section I. A new term, the $\pi d V_{w}$ term, has been introduced in which the new extensive variable $\left(V_{w}\right)$ is the same as an existing variable in the equation. In the gravitational case, this led to equation $(1-163)$,

$$
d G=\sum\left(\mu_{i}+M_{i} \phi\right) d n_{i} \quad(T, P \text { constant })
$$

or

$$
\left(\frac{\partial G}{\partial n_{i}}\right)_{T, P, n_{i}}=\mu_{i}+M_{i} \phi
$$

In the electrochemical case the result was equation (1-235), which may be written in the form

or

$$
d G=\sum\left(\mu_{i}+z_{i} F_{y} \psi\right) d n_{i} \quad(T, P \text { constant })
$$

$$
\left(\frac{\partial G}{\partial n_{i}}\right)_{T, P, n_{j}}=\mu_{i}+z_{i} F_{y} \psi
$$

In each case, the significance of the individual terms $\mu_{i}$ and $\phi$ or $\mu_{i}$ and $\psi$ had to be determined independently. For gravitation, $\phi$ can be determined by direct physical measurement, and the significance of $\mu_{i}$ is not altered by the field. In electrochemistry, however, $\mu_{i}$ and $\psi$ cannot be given thermodynamic significance.

In the case at hand, the physical significance of $P_{w}$ is unambiguous. It may next be shown that this is also true of $\pi$. Day and Forsythe (in press) show that

$$
-\left(P_{w}-P_{A}\right) d V_{w}+\left(P-P_{A}\right) d V-\pi d V_{w}=0
$$


We have already assumed equation (5-74), so that

$$
-\left(P_{w}-P_{A}\right) \bar{V}_{w} d n_{w}+\left(P-P_{A}\right) d V-\pi \bar{V}_{w} d n_{w}=0
$$

Dividing by $d n_{w}$ at constant confining pressure, we obtain

and

$$
-\left(P_{w}-P_{A}\right) \bar{V}_{w}+\left(P-P_{A}\right)\left(\frac{\partial V}{\partial n_{w}}\right)_{P}-\pi \bar{V}_{w}=0
$$

$$
\pi=\left(\frac{P-P_{A}}{\bar{V}_{w}}\right)\left(\frac{\partial V}{\partial n_{w}}\right)_{P}+\left(P_{A}-P_{w}\right)
$$

The right-hand side contains only macroscopic properties and thus gives the physical significance of $\pi$. Notice that for a saturated soil in which geometry effects may be assumed absent, $\left(\partial V / \partial n_{w}\right)_{P}=\bar{V}_{w}$, so that

$$
\pi=P-P_{w}
$$

This is known as the Terzaghi equation.

In nonswelling soils, $P=P_{A}$, and

$$
\pi=P_{A}-P_{w}
$$

If $P_{A}=P^{\circ}, \pi$ is the negative of the tension.

\section{VERTICAL DISTRIBUTION}

Babcock and Overstreet (1957b) applied equation (5-9) to the vertical distribution of water in soil above a water table. For a salt-free soil at constant temperature, a combination of equations (1-167) and (5-9), results in

$$
\bar{V}_{w} d P_{e}+(\partial \mu / \partial \theta)_{P} d \theta+M g d h=0
$$

Solving for the vertical distribution, we obtain

$$
\frac{d \theta}{d h}=\frac{M g+\bar{V}_{w}\left(\frac{d P_{e}}{d h}\right)}{(\partial \mu / \partial \theta)_{P}}
$$

This result differs from Buckingham's equation (1907) by the $d P_{e} / d h$ term. Babcock and Overstreet (1957b) concluded that where the "hydrostatic pressure" gradient in the soil water is governed by the ordinary condition,

$$
\begin{aligned}
d P & =-\rho g d h \\
& =-\left(\frac{M}{\bar{V}_{w}}\right) g d h
\end{aligned}
$$

Then equation (5-96) yields $d \theta / d h=0$. It was further concluded that ". . there is the possibility of capillary fringes extending to greater heights than might be anticipated from curves determined with tensiometers or pressure plates." This conclusion has been criticized by Collis-George (1961), whose conclusions the writer now believes to be correct in many respects. A re-evaluation of the problem will be attempted. 
Imagine a column of dry, uniformly packed, salt-free soil in a cylinder with rigid walls. The soil may be supported at the bottom by a rigid screen which holds the particles but will allow water to pass freely. The bottom end is now immersed in a large free water table, and subsequently sealed at the top. The column is allowed to come to equilibrium at a uniform temperature.

For a nonswelling soil, the equilibrium vertical distribution is given by equation (5-96). In any zone of unsaturation, the pressure gradient is merely the vertical pressure gradient in the gaseous phase which is negligible. Thus, Buckingham's equation is regained. In any zone of saturation, the pressure is the macroscopic pressure exerted by the walls on the soil. It is the same as the "confining pressure" exerted by a piston in the previous section. The point overlooked by Babcock and Overstreet $(1957 b)$ is that as water enters the soil, no force can be exerted against the wall unless there is a displacement of particles in space, ${ }^{7}$ that is, by swelling. The treatment, then, must be entirely parallel to the treatment of excess pressure in spheres in Section I, where the pressure external to the sphere, rather than the pressure within the sphere, entered into equation (1-218). Therefore, in the saturated zone of a nonswelling soil, the vertical distribution would still be given by Buckingham's equation. Consequently, the vertical distribution could be predicted from the moisture characteristic.

For a swelling soil, there appears to be agreement (Bolt and Frissel, 1960; Collis-George, 1961) that Buckingham's equation will not give the vertical distribution. Geometric terms must be included in the expression for chemical potential, so that

$$
\frac{d \theta}{d h}=-\frac{M g+\bar{V}_{w}\left(\frac{d P_{e}}{d h}\right)+\left(\frac{\partial \mu}{\partial V}\right)\left(\frac{d \chi}{d h}\right)}{(\partial \mu / \partial \theta)}
$$

So far as the writer is aware, no means exists for the prediction of $d P_{e} / d h$ or $d \chi / d h$. These terms must be measured experimentally by measuring the pressure exerted by the confining wall and the geometry term as functions of height. Clearly, an experimental check of equation (5-99) would be difficult, and as indicated by Collis-George (1961), the results would depend upon the manner in which the vertical column is brought to equilibrium.

\footnotetext{
${ }^{7}$ First suggested to the writer by R. D. Miller.
} 


\section{LITERATURE CITED}

Alexander, A. E., and P. Johnson

1949. Colloid science. Oxford Univ. Press, London. 837 p.

Anderson, D. M., and P. F. Low

1958. The density of water adsorbed by lithium, sodium, and potassium bentonite. Soil Sci. Soc. Am. Proc. 22:99-102.

Argersinger, W. J., A. W. Davidson, and O. D. Bonner

1950. Thermodynamics and ion exchange phenomena. Kansas Acad. Sci. Trans. 53:404-410.

BABCOCK, K. L.

1960. Some characteristics of a model Donnan system. Soil Sci. 90:245-252.

Babcock, K. L., L. E. Davis, and Roy Overstreet

1951. Ionic activities in ion-exchange systems. Soil Sci 72:253-260.

BABCOCK, K. L., and Roy OverstreEt

1955. The thermodynamics of soil moisture: A new approach. Soil Sci. 80:257-263.

1957a. The extra-thermodynamics of soil moisture. Soil Sci: 83:455-464.

1957b. A note on the "Buckingham" equation. Soil Sci. 84:341-343.

BenerJee, Somir

1959. Some aspects of salt-affected soils of West Bengal. Soil Sci. 88:45-50.

Bockris, J. O'M. [ed.]

1959. Modern aspects of electrochemistry. Academic Press, New York. 416 p.

BoLt, G. H.

1955a. Ion adsorption by clays. Soil Sci. 79:267-276.

1955b. Analysis of the validity of the Gouy-Chapman theory of the electric double-layer. J. Colloid Sci. 10:206-218.

Bolt, G. H., and J. M. Frissel

1960. Thermodynamics of soil moisture. Netherlands J. Agr. Sci. 8:57-78.

Bolt, G. H., and R. D. Miller

1955. Swelling pressures of illite suspensions. Soil Sci. Soc. Am. Proc. 19:285-288.

Bolt, G. H., and M. PeEch

1953. The application of the Gouy theory to soil-water systems. Soil Sci. Soc. Am. Proc. 17: 210-213.

Bower, C. A.

1959. Cation-exchange equilibria in soils affected by sodium salts. Soil Sci. 88:25-35.

Bower, C. A., and J. O. Goertzen

1955. Negative adsorption of salt by soils. Soil Sci. Soc. Am. Proc. 19:147-151.

BuCKingham, E. A

1907. Studies on the movement of soil moisture. U. S. Dept. Agr. Bull. 38. 61 p.

Collis-George, N.

1961. Free energy considerations in the moisture profile at equilibrium and effect of external pressure. Soil Sci. 91:306-311.

Davis, L. E.

1942. Significance of Donnan equilibria for soil colloidal systems. Soil Sci. 54:199-219.

$1950 a$. Ionic exchange and statistical thermodynamics. I. Equilibria in simple exchange systems. J. Colloid Sci. 5:71-79.

1950b. Ionic exchange and statistical thermodynamics. II. Equilibria in irregular systems. J. Colloid Sci. 5: 107-113.

Davis, L. E., and J. M. RIBLE

1950. Monolayers containing polyvalent ions. J. Colloid Sci. 5:81-83.

DaY, P. R., and W. M. Forsythe

1962. Effect of isotropic confining pressure on the hydrostatic pressure of water in unsaturated soil. Science 136:779-780.

Donnan, F. G.

1911. Theorie der Membrangleichgewichte und Membranpotentiale bei Vorhandensein von nicht dialysierenden Electrolyten. Ztschr. f. Electrochem. 17:572-581.

Donnan, F. G., and E. A. Guggenheim

1932. Die genaue Thermodynamik der Membrangleichgewichte. Ztschr. f. Phys. Chem. $162(\mathrm{~A}): 346-360$. 
Eriksson, E.

1952. Cation exchange equilibria on clay minerals. Soil Sci. 74:103-113.

Forsythe, W. M.

1962. The effect of an applied isotropic pressure on soil water pressure of unsaturated soils.

Ph.D. Dissertation. University of Calif. 85 p.*

Fowler, R. H., and E. A. GugGenheim

1939. Statistical thermodynamics. Macmillan Co., New York. 693 p.

Gaines, G. L., and H. C. Thomas

1953. Adsorption studies on clay minerals. II. J. Chem. Phys. 21:714-718.

GAPON, E. N.

1933. On the theory of exchange adsorption in soils. [In Russian] U.S.S.R. J. Gen. Chem. $3: 144$.

Gouy, C.

1910. Sur la constitution de la charge electrique a la surface d'un electrolyte. J. Phys. 9(4): $457-468$.

Guggenheim, E. A.

1949. Thermodynamics. An advanced treatment for chemists and physicists. Interscience Pub. Inc., New York. 394 p.

HARned, H. S., and B. B. Owens

1958. The physical chemistry of electrolytic solutions. 3d ed, Reinhold Pub. Corp., New York. 645 p.

Jenny, H., D. E. Williams, and N. T. Coleman

1950. Concerning the measurement of $\mathrm{pH}$, ion activities, and membrane potentials in colloidal systems. Science 112:164-167.

KLINE, J. J., and F. O. Koenig

1957. The state principle-some general aspects of the relationships among the properties of systems. J. Appl. Mechanics 24:29-34.

Krishnamoorthy, C., L. E. Davis, and R. Overstreet

1949. Ionic exchange equations derived from statistical thermodynamics. Science 108:439-440.

Krishnamoorthy, C., and R. Overstreet

1950a. An experimental evaluation of ion-exchange relationships. Soil Sci. 69:41-53.

1950b. Behavior of hydrogen ion-exchange reactions. Soil Sci. 69:87-93.

LAGerwerfF, J. V., and G. H. Bolt

1959. Theoretical and experimental analysis of Gapon's equation for ion exchange. Soil Sci. 87:217-222.

Lewis, G. C., and R. L. Juve

1956. Some effects of irrigation water quality on soil characteristics. Soil Sci. 81:125-137.

Lewis, G. N., and M. RANDALL

1923. Thermodynamics and the free energy of chemical substances. McGraw-Hill Book Co., New York. 653 p.

Longenecker, D. E., and P. J. Lyerly

1959. Chemical characteristics of soils of West Texas as affected by irrigation water quality. Soil Sci. 87:207-216.

Low, P. F.

1951. Force fields and chemical equilibrium in heterogeneous systems with special reference to soils. Soil Sci. 71:409-418.

1955. Effect of osmotic pressure on diffusion rate of water. Soil Sci. 80:95-100.

MacInnis, D. A.

1939. The principles of electrochemistry. Reinhold Pub. Corp., New York. 476 p.

Marshall, C. E.

1958. Physico-chemical properties of solid-liquid interfaces in soil systems. Soil Sci. Soc. Am. Proc. 22:486-490.

Marshall, C. E., and C. A. Krinbill

1941. The clays as colloidal electrolytes. J. Phys. Chem. 46:1077-1090.

Mattson, J.

1926. The laws of colloidal behavior. I. Soil Sci. 28:179-220.

Mattson, S., and K. G. Larsson

1946. The laws of soil colloidal behavior: XXIV. Donnan equilibria in soil formation. Soil Sci. 61:313-330.

\footnotetext{
* Microfilm copies may be purchased from the University of California Library Photographic Service, Berkeley.
} 
Overbeek, J. Th. G.

1956. The Donnan equilibrium. Progr. in Biophysics 6:58-94.

Peech, M., R. A. Olson, and G. H. Bolt

1953. The significance of potentiometric measurements involving liquid junction in clay and soil suspensions. Soil Sci. Soc. Am. Proc. 17:214-218.

Peech, M., and A. D. ScotT

1950. Determination of ionic activities in soil-water systems by means of the Donnan membrane equilibrium. Soil Sci. Soc. Am. Proc. 15:115-119.

Richards, L. A. [ed.]

1954. Diagnosis and improvement of saline and alkali soils. U. S. Dept. Agr. Handbook 60. $160 \mathrm{p}$.

Schofield, R. K.

1947. Calculations of surface areas from measurements of negative adsorption. Nature 160: 408-410.

Taвikh, A. A., I. Barshad, and R. Overstreet

1960. Cation exchange hysteresis in clay minerals. Soil Sci. 90:219-226.

TAGakI, S.

1959. Theory of freezing point depression of soil water, and a note on the extrathermodynamics of soil moisture. Soil Sci. 88:25-31.

TAYlor, S. A.

1958. The activity of soil water. Soil Sci. 86:83-90.

TAylor, S. A., and J. E. Box

1961. Influence of confining pressure and bulk density on soil water matric potential. Soil Sci. 91:6-10.

Taylor, S. A., and G. L. Stewart

1960. Some thermodynamic properties of soil water. Soil Sci. Soc. Am. Proc. 24:243-247.

VAN der Molen, W. H.

1958. The exchangeable cations in soils flooded with sea water. Ph.D. thesis, Agricultural University, Wageningen, Netherlands.

Vanselow, A. P.

1932. Equilibria of the base exchange reactions of bentonites, permutities, soil colloids and zeolites. Soil Sci. 33:95-113.

Verwey, E. S. W., and J. Th. G. Overbeek

1948. Theory of the stability of lyophobic colloids. Elsevier Pub. Co., Inc., New York. 205 p.

Whitney, R. S., and M. Peech

1952. Ion activities in sodium clay suspensions. Soil Sci. Soc. Am. Proc. 16:117-122.

WIKLANDER, L.

1955. Cation and anion exchange phenomena. Chapt. 4. In: Chemistry of the Soil. F. E. Bear [ed.]. Reinhold Pub. Corp., New York. 


\section{CONTENTS}

Section I. Pertinent physical chemistry . . . . . . 419

A system of chemical thermodynamics . . . . . . 419

Solutions . . . . . . . . . . . . 430

Systems involving other variables . . . . . . . . 440

Section II. Donnan theory . . • . . . . . . . . . 458

Section III. Double-layer theory . . . . . . . . . . 471

Gouy theory ... . . . . . . . . . . 4 471

Chemical potentials. . . . . . . . . . . . . 481

Applicability to soil systems . . . . . . . . . 495

Section IV. Ion-exchange theory . . . . . . . . . . 496

Ion-exchange equations .. . . . . . . . . 496

Applicability to soil systems . . . . . . . . . . 507

Section V. Thermodynamics of water in soil systems . . . 524

Introduction . . . . . . . . . . . . . . . 524

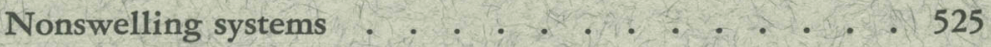

Swelling systems . • • • . . . • . . . . . . 531

Relation between thermodynamic and mechanical treatments . . . . . . . . . 535

Vertical distribution .. . . . . . . . . . 538 
The journal HILGARDIA is published at irregular intervals, in volumes of about 650 to 700 pages. The number of issues per volume varies.

Single copies of any issue may be obtained free, as long as the supply lasts; please request by volume and issue number from:

$$
\begin{aligned}
& \text { Agricultural Publications } \\
& 207 \text { University Hall } \\
& \text { University of California } \\
& \text { Berkeley 4, California }
\end{aligned}
$$

The limit to nonresidents of California is 10 separate titles. The limit to California residents is 20 separate titles.

The journal will be sent regularly to libraries, schools, or institutions in one of the following ways:

1. In exchange for similar published material on research.

2. As a gift to qualified repository libraries only.

3. On a subscription basis $-\$ 7.50$ a year paid in advance. All subseriptions will be started with the first number issued during a calendar year. Subscribers starting during any given year will be sent back numbers to the first of that year and will be billed for the ensuing year the following January. Make checks or money orders payable to The Regents of The University of California; send payment with order to Agricultural Publications at above address. 